\title{
Cone Penetration Testing in Polar Snow
}

\author{
Adrian Bruce McCallum \\ Gonville and Caius College
}

January 2012

This dissertation is submitted for the degree of Doctor of Philosophy. 


\section{Declaration}

This dissertation is the result of my own work and includes nothing which is the outcome of work done in collaboration except where specifically indicated in the text. 


\section{Dedication}

This work is dedicated to my grandfather, Mr Rodney Frank, 1914 - 2010.

"Play up! Play up! And play the game!" (Newbolt, 1892) 


\section{Acknowledgements}

This thesis and the research supporting it was only possible through the support of many individuals and numerous organisations, some of whom I will attempt to acknowledge here. No doubt there will be omissions and to those I have neglected to thank, my apologies; this work would not have come about without your assistance.

Firstly I must thank the Menzies Association because without their support (in conjunction with the Australian Government and the British Council) my family and I would not have had the means to support ourselves in Cambridge. Additional financial support came from the Cambridge Commonwealth Trusts, thence additionally from the Transglobe Expedition Trust, whose acknowledgement of my specific requirements are most appreciated.

The Director of the Scott Polar Research Institute (SPRI) Julian Dowdeswell entertained the possibility of my attendance at SPRI in 2006, and thanks must go to him for his enthusiasm and support from before my arrival and throughout my period at the Institute. Julian's offsiders, initially Liz Crilly and thence Kate Gilbert, both wonderful ladies, were fantastic in addressing the needs of an oft' demanding retired Major, and I thank them both for all they did in smoothing my path. SPRI's public presence is typically Claire Lampitt, and I can't thank Claire enough for her cheerful assistance in all matters through out my time; she's a good lass.

The staff of the SPRI library, Heather, Shirley, Mark and Rebecca were all terrific in their assistance over my time at SPRI; no request was too much, and helpful advice was always forthcoming. Additional library support was given by Michelle Beane, librarian at the Cold Regions Research and Engineering Laboratories (CRREL) in Hanover, New Hampshire, who happily and patiently dealt with my near-endless requests for CRREL publications. The Engineering library too was a favourite haunt, and I must thank Louise Yirrell and Diane FitzMaurice.

For my initial field testing in Greenland I must thank Alun Hubbard for providing me with the opportunity, and Fugro B.V. Netherlands, for kindly lending me their snow micro-penetrometer for this journey; specific thanks there to Peter Looijen \& Sebastiaan Smeitz. Thanks to Rickard Pettersson too, for his example of professionalism in the field and his continued advice on all things GPR.

For my initial research 'dabbling' in Cambridge I must thank Bill O'Neill for his time and assistance in our initial laseing trials, Chris Knight of Engineering for address- 
ing my endless equipment queries, and Malcolm Bolton and Stuart Haigh, also from Engineering, for their assistance on possible centrifuge testing. Kenichi Soga, Sarah Springman, Martin Fahey, Branko Ladanyi and Clive Dalton from Cambridge Insitu also gave me valuable time and advice as I found my way. Bob Hawley and George Blaisdell also deserve my thanks, for encouraging me in my work, and for providing exterior guidance on all manner of snow and polar matters. It truly is an international field, and I look forward to future collaboration.

My major field research in Antarctica would not have been possible without the assistance of Lankelma and Gardline, and the British Antarctic Survey (BAS); Lankelma / Gardline provided the equipment and BAS got me there and employed me. Thanks must go to Eric Zon, Brian Georgious and Andy Barwise of Lankelma / Gardline who initially considered my 'pitch' and who subsequently came through with a great bit of kit, UK11, which proved most effective in conducting CPT in Antarctica. Ongoing technical assistance was provided by Roi Santos, who taught me a lot about CPT, and always took the time to answer my simple questions, essentially keeping my testing programme on track; thank you Roi. UK11 came to be through the engineering skills of Richard Boutell and Michael 'Fred' Davies, with additional assistance from Andrew Hewitt and Will Shearer. Thank you all to a great 'box' prepared in quick time.

From BAS I must thank David Blake and Karl Tuplin who considered the value of my research and enabled me to serve with BAS at Halley V, and Martin Bell and Ben Norrish both were fantastic and patient in addressing all manner of snow mechanics/logistics/transport requirements that I had, both during my time at Halley, and throughout my whole $\mathrm{PhD}$; always shall they remain fearful of my email in their inbox. Whilst at Halley I had the opportunity to work with a great bunch of blokes who I'd happily serve with again, anywhere, anytime. As the 'Ops Beaker' I was fortunate to be able to exist within both the Scientific and Operational worlds, whilst existing within the garage; a wonderful mix. To Steve Pollitt, Andy Mac, Brian Brock, Colin 'Shifty' Reston, Tim Gee, Ryan Andersen and David Goulden; thank you for showing me the ropes and including me in the team. We had a great time and did good work, thank you.

For the additional equipment required for my field-testing I must thank Adrian Hayes and Chris Rolfe of Geography. Adrian gave me a lot of time and I learnt a lot from him. He was always happy to answer my naive questions and was always happy to help. Chris too was always willing and able and kindly provided me with much of the additional paraphernalia that I required.

For my laboratory testing at UCL I must thank Peter Sammonds who accommodated my request for assistance, and kindly allowed me to share his facility. Testing was only possible through the wonderful assistance and ongoing attention of Ben Lishman and Steve Boon, who got the equipment running, kept it running and assisted in data extraction. This work was only possible because of their time and effort on my behalf; 
thank you. Thanks too, to a man who rendered very willing and helpful assistance late in the game; Larry Conyers in Denver, who helped me drag some amplitudes out of my unwilling GPR .dzt files.

To my colleagues at SPRI, both student and staff I must acknowledge your continued enthusiasm and support of both my research and my day-to-day travails, and your continued friendship. To Ian Willis, Gareth Rees, Neil Arnold, Toby Benham and Poul Christoffersen; thank you for your professional advice on all manner of things, and for your ready acceptance of me into your academic circles; such continued humble support was always appreciated. To Ruth Mugford, Kelly Hogan, Cameron Rye, Martin O'Leary, Narelle Baker and Alison Banwell; thanks guys for all your friendship and support. We had a lot of fun and may your future careers bring you much satisfaction.

There's one lady who's done more than any one to keep this $\mathrm{PhD}$ on track, and that's Liz Morris. I harassed Liz some years back on the merits of a PhD in glaciology, and then before she knew it there I was, lobbed on her lap in need of much guidance and advice. Liz had to keep the ship on course, and ensured it was fuelled: mentally, physically, spiritually, financially. Liz attempted to continually address all our needs as a family and truly provided a broad and solid foundation upon which I could build this work. Thank you Liz. I cherish you as a friend and colleague very much; I value and respect you very much, and I hope that we keep in touch, as perhaps that bucking skidoo seat gets a bit hard. As Campbell would say, "You're a good woman."

Of course, the only reason that I'm here completing this work is because of the continued love of my family; my parents Stan and Marnie, who've supported me in all that I've done, but more importantly my dear wife Claire and our two terrors, Campbell and Bill. It's been a long and at times challenging journey, but we've stuck together and made it through; here's to many more adventures down the track. Thank you for allowing me to pursue this challenge, for your care during all my absences, and for your continued steering of our family. You're a treasure and I'm a lucky man; thank you.

So to all of you again I say, thank you, and I hope we meet again down the track..... 


\begin{abstract}
Innovative Cone Penetration Testing (CPT) using adapted commercial CPT equipment was conducted in Antarctica in early 2010 in an attempt to assess the strength of polar snow; additionally, application of CPT data was considered, particularly in estimating surface bearing capacity. Almost 100 CPT tests were carried out and both qualitative and quantitative analysis of data was undertaken. Additional supporting testing included snow density assessment, snow strength assessment, extrapolation of CPT data via Ground Penetrating Radar (GPR) and preliminary mini-cone penetrometer testing in Greenland.

Analysis of results revealed that assessing the strength of polar snow via CPT is affected by numerous factors including penetration rate, cone size/shape and snow material properties, particularly compaction of the snow undergoing penetration. A density-dependant relationship between CPT resistance and snow shear strength was established, and methods for estimating surface bearing capacity directly from CPT in homogeneous and layered polar snow were proposed.

This work applied existing technology in a new material and shows that CPT can be used efficiently in polar environs to provide estimates of snow shear strength and surface bearing capacity, to depths of $10 \mathrm{~m}$ or more.
\end{abstract}




\section{Glossary}

Allowable bearing capacity The ultimate bearing capacity divided by a factor of safety and/or the maximum bearing capacity tolerable that will cause a limit value of settlement.

California Bearing Ratio (CBR) A simple penetration test developed to evaluate the strength of road subgrades, conducted by causing a plunger of standard area to penetrate a soil sample, either in a laboratory or on site.

Cavity Expansion (Theory) The pressure at the wall of an expanding cavity in an infinite soil mass approaches a limiting value at large strains, and this, for the case of a spherical cavity, is used to interpret cone resistance of the cone penetration test (Russell, 2004).

Compacted Zone Area possibly formed ahead of the cone consisting of fractured and compacted particles (ice grains, bonds).

Cone Angle Angle subtended between the tip of a cone.

Cone Factor Empirically derived parameter relating tip resistance (obtained via CPT) with (undrained) shear strength.

Confining Pressure In-situ stress at depth, equivalent to (total) horizontal stress.

Constrained Modulus (D') Elastic modulus for one-dimensional loading.

CPT Cone Penetration Testing.

Creep Ductile deformation of ice (and thus snow) primarily through the movement of crystallographic basal planes due to load applied at low strain rates .

Effective Area The extended end-bearing area of the cone or plate during penetration, typically increased owing to the carriage of fractured ice grains.

Failure When the material can no longer resist the applied forces or becomes otherwise unfit for purpose (subject to definition). 
Fluidisation State where snow (or material) has been disturbed or processed to such an extent that it exhibits nil or negligible strength, and exists as a loose particulate.

FoS Factor of Safety. In engineering practice a Factor of Safety (typically equal to two or three, depending on application) is usually applied to calculated strengths, bearing capacities etc. in an effort to define a safe working load for a system such that failure should not occur.

General shear failure When a clearly defined plastic yield slip surface forms under a footing and develops outward towards one or both sides and eventually to the ground surface.

Geomaterials Materials formed of the earth.

Layer A distinct stratum of snow that differs in at least one respect from the strata above and below. Differences may include density, grain size, microstructure, grain size, grain shape or hardness.

Local shear failure When significant vertical movement occurs before any noticeable development of shear planes occurs; shear planes fail to extend to the ground surface.

Microstructure Strictly defined as the configuration of the ice and air spaces within the snow incorporating, density, porosity, specific surface area, curvature, tortuosity and coordination number. However, within this work I choose to define it more specifically as the size and number of bonds between ice grains within the snow.

Plug Mass of compacted snow possibly formed ahead of the cone/plate during penetration. See Compacted Zone.

Porosity Numerically equal to one minus relative density.

Pressure Bulb Extent of stress distribution into a medium under an applied surface load.

Punching shear failure Considerable vertical movement occurs with slip surfaces reduced to vertical planes adjacent to the sides of the footing.

Regelation The phenomenon of melting under pressure and freezing again when the pressure is reduced.

Sensing The cone 'senses' ahead of itself during penetration. This is due to the elastic response of the material and varies with material stiffness. 
Sensitivity The ratio of undisturbed peak undrained shear strength to totally remolded undrained shear strength, usually pertaining to clays. High sensitivity suggests low remoulded shear strength (strength upon fracture and remoulding); considered negligible post-fracture in snow.

Shear Modulus (G) Slope of shear stress versus shear strain curve; strictly, when the confining stress equals zero.

Signal Analysis The extraction of information from complex signals in the presence of noise, followed by analysis using various methods.

Sintering The bonding of ice grains (or more generally particles) under heat and/or compression.

Small-strain Shear Modulus (Gmax) Fundamental stiffness at the initial state of the material; applicable to the initial non-destructive range of strains.

SMPT Snow Micro PeneTrometer.

Stick-slip behaviour When surfaces moving relative to one another alternate between sticking to each other and sliding over each other, with a corresponding change in the frictional force.

Stiffness Area-dependant measure of resultant displacement due to applied force. (Use of modulus is preferable as it is an inherent material property and by definition is not area dependant).

Stratigraphy The definition and description of layers within the snowpack.

Subtraction Cone A cone that measures the total force on the penetrometer (sleeve + tip) and the tip resistance, and then calculates sleeve friction by subtracting tip resistance from the total force.

Toughness Resistance to brittle fracture.

Ultimate bearing capacity The theoretical maximum pressure which can be supported without failure.

Note: Where appropriate, terms within this Glossary have been defined consistent with The International Classification for Seasonal Snow on the Ground (Colbeck et al., 1990) and Cone Penetration Testing in Geotechnical Practice (Lunne et al., 1997). 


\section{Contents}

Glossary vii

1 Introduction $\quad 1$

1.1 Motivation . . . . . . . . . . . . . . . . . . . . 1

1.2 Purpose of my research . . . . . . . . . . . . . . . . . . 2

1.3 Significance of this thesis . . . . . . . . . . . . . . . 3

1.4 Thesis Outline . . . . . . . . . . . . . . . . . . 4

2 Review 5

2.1 The Nature of Snow . . . . . . . . . . . . . . . . . . 5

2.1.1 How does it form? . . . . . . . . . . . . . . . 5

2.1.2 How does snow behave? . . . . . . . . . . . . . . . . . . 10

2.1.3 Other analogues for snow . . . . . . . . . . . . . 13

2.1.4 Behaviour during penetration . . . . . . . . . . . . . . 14

2.2 Strength testing in snow . . . . . . . . . . . . . . . . . . . . 16

2.2.1 Hardness and Resistance . . . . . . . . . . . . . . . 18

2.2 .2 Types of strength . . . . . . . . . . . . . . . . . . . 20

2.2.3 Cohesion from CPT resistance . . . . . . . . . . . . . . 21

2.2.4 Effect of sample size . . . . . . . . . . . . . . . . . . . 21

2.3 Penetrative testing in snow . . . . . . . . . . . . . . . 22

2.3.1 Rammsonde . . . . . . . . . . . . . . . . . . 23

2.3.2 Thermo Resistograph . . . . . . . . . . . . . . . . . 23

2.3.3 Snow Micro Penetrometer (SMPT) . . . . . . . . . . . . . . . 24

2.3.4 Other penetrative instruments . . . . . . . . . . 25

2.3.5 The Cone Penetration Test $(\mathrm{CPT}) \ldots \ldots . \ldots 25$

2.4 Summary . . . . . . . . . . . . . . . . . . . . . . 27

3 Methods 28

3.1 Fugro mini penetrometer testing . . . . . . . . . . . . . . . . . . . . 29

3.2 Cone Penetration Testing _ . . . . . . . . . . . . . . . . . . . . . . . 32

3.2.1 Location \& Equipment . . . . . . . . . . . . . . . . . . . . 32

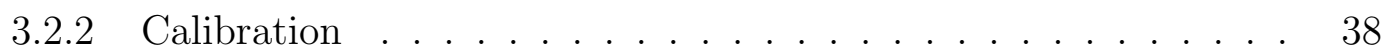


3.2.3 Specific questions to be addressed in performing CPT . . . . . . 39

3.2.4 Testing Process \& Problems Encountered . . . . . . . . . . . . . 40

3.3 Density Assessment . . . . . . . . . . . . . . . . . . . . 41

3.4 Confined compressive strength testing . . . . . . . . . . . . . . . . . 43

3.5 Strength Testing (Shear Frame) . . . . . . . . . . . . . . . . . . . . . . 44

3.6 Pressure Bulb Testing . . . . . . . . . . . . . . . . . . . 48

3.7 Ground Penetrating Radar (GPR) . . . . . . . . . . . . . . . . . 52

3.8 Summary . . . . . . . . . . . . . . . . . . . . . . 55

4 Results \& Analysis (A) - Fugro mini-penetrometer 56

4.1 Distance for resistance to be mobilised . . . . . . . . . . . . . . . 57

4.2 Tip resistance \& sleeve friction . . . . . . . . . . . . . . . . . 58

4.3 Rate Effects . . . . . . . . . . . . . . . . . . . . . 60

4.4 Age hardening . . . . . . . . . . . . . . . . . . . 61

4.5 Variation with density . . . . . . . . . . . . . . . 61

4.6 Cone effective area . . . . . . . . . . . . . . . . . . . . . . 64

4.7 Formation of compacted zone . . . . . . . . . . . . . . 64

4.8 'Chipping' of unconfined blocks . . . . . . . . . . . . . . . 66

4.9 Summary . . . . . . . . . . . . . . . . . . . . . . . 68

5 Results \& Analysis (B) - Cone Penetration Testing 69

5.1 CPT results . . . . . . . . . . . . . . . . . . . . . . 69

5.2 Basic Signal Analysis . . . . . . . . . . . . . . . . . . . 70

5.2.1 Tip Resistance . . . . . . . . . . . . . . . 71

5.2 .2 Sleeve Friction . . . . . . . . . . . . . . . . . . 72

5.2 .3 Friction Ratio . . . . . . . . . . . . . . . . . . . . 73

5.2 .4 Pore Pressure . . . . . . . . . . . . . . . . . . . . 74

5.3 Variation of signals with depth . . . . . . . . . . . . 76

5.3.1 Tip Resistance . . . . . . . . . . . . . . 76

5.3 .2 Sleeve Friction . . . . . . . . . . . . . . . . . . . 76

5.4 Variation of tip resistance with sleeve friction . . . . . . . . . . . 77

5.5 Spatial Variability . . . . . . . . . . . . . . . . . . . 77

5.6 Qualitative spatial comparison of CPT data . . . . . . . . . . . 80

5.7 Effect of grain size . . . . . . . . . . . . . . . . . . 80

5.8 Effect of penetration rate . . . . . . . . . . . . . . . 82

5.8 .1 Tip Resistance . . . . . . . . . . . . . . . . 84

5.8 .2 Sleeve Friction . . . . . . . . . . . . . . . . . . . . . 85

5.8 .3 Friction Ratio . . . . . . . . . . . . . . . . 86

5.8 .4 Summary . . . . . . . . . . . . . . . . 87

5.9 Effect of penetrometer size . . . . . . . . . . . . . . . 87

5.9 .1 Cone size and strain rate . . . . . . . . . . . . . . . . . 88 
5.10 Effect of cone shape . . . . . . . . . . . . . . . . . . . . . . 89

5.11 Cone Effective Area . . . . . . . . . . . . . . . . . . . . 90

5.12 Formation of compacted zone . . . . . . . . . . . . . . . 92

5.13 Sleeve Friction reassessment . . . . . . . . . . . . . . . . . . . . . . 95

5.14 'Sensing' ahead of the penetrometer . . . . . . . . . . . . . . . . 96

5.15 Effect of confining pressure . . . . . . . . . . . . . . . . . . 99

5.15 .1 Tip Resistance . . . . . . . . . . . . . . . . . . 100

5.15 .2 Sleeve Friction . . . . . . . . . . . . . . . . . . . . . 101

5.16 Pressure melting . . . . . . . . . . . . . . . . . . . 102

5.17 Dissipation Tests . . . . . . . . . . . . . . . . . . 102

5.18 Cyclic Testing . . . . . . . . . . . . . . . . . . . 104

5.19 Interpretation via Standard Soil Charts . . . . . . . . . . . . . . 105

5.19.1 Comparison with cemented sands . . . . . . . . . . 106

5.20 Snow microstructure from CPT . . . . . . . . . . . . . . . . 107

5.21 Summary . . . . . . . . . . . . . . . . . . . . . . 109

6 Results \& Analysis (C) - Supplementary subsurface investigations 110

6.1 Density Assessment . . . . . . . . . . . . . . . . . . . . . . . . 110

6.2 Confined compressive strength testing . . . . . . . . . . . . . . . . . . 112

6.3 Strength Testing (Shear Frame) . . . . . . . . . . . . . . . . . 115

6.4 Pressure Bulb Testing . . . . . . . . . . . . . . . . . 119

6.5 Ground Penetrating Radar (GPR) . . . . . . . . . . . . . . . . . 122

6.6 Summary . . . . . . . . . . . . . . . . . . . . . . . . 125

7 Stratigraphy \& strength from CPT $\quad 126$

7.1 CPT and stratigraphy . . . . . . . . . . . . . . . . 126

7.1.1 Incorporation of friction sleeve to resolve stratigraphy . . . . . . 130

7.1.2 Stratigraphy resolution using a flat plate . . . . . . . . . . . 131

7.2 Variation with density . . . . . . . . . . . . . . . . . 132

7.2.1 Tip . . . . . . . . . . . . . . . . . . 132

7.2 .2 Sleeve friction . . . . . . . . . . . . . . . . . . 134

7.3 Comparison of tip resistance with hardness . . . . . . . . . . . . 135

7.4 Snow strength estimation . . . . . . . . . . . . . . . . . 138

7.4.1 Existing methods for deducing snow strength . . . . . . . . . 140

7.4.2 Conceptual Evaluation . . . . . . . . . . . . . . . . . 143

7.4.3 Quantification \& verification . . . . . . . . . . . . 151

7.4.4 Other considerations . . . . . . . . . . . . . . . . 152

7.4.5 Estimating strength solely from CPT . . . . . . . . . . 153

7.5 Comparison with strength data . . . . . . . . . . . . . 154

7.5.1 Comparison with statistical micromechanical model . . . . . . . 156

7.5.2 Estimating sleeve friction . . . . . . . . . . . . . . . 157 
7.6 Summary . . . . . . . . . . . . . . . . . . . . . . . . . . . . . . . . . . 158

8 Application of CPT data $\quad 159$

8.1 Definitions . . . . . . . . . . . . . . . . . . . . 159

8.1 .1 Types of failure . . . . . . . . . . . . . . 160

8.1.2 Bearing capacity from CPT . . . . . . . . . . . 161

8.2 Indirect assessment of bearing capacity . . . . . . . . . . . . . . 161

8.2.1 Homogeneous snow - ultimate bearing capacity . . . . . . . . . 161

8.2.2 Homogeneous snow - allowable bearing capacity . . . . . . . . . 164

8.2 .3 Layered snow . . . . . . . . . . . . . . . . 166

8.2.4 Comparison of stress with strength . . . . . . . . . . . . 173

8.3 Direct Assessment of Bearing Capacity . . . . . . . . . . . . . . . . . . 177

8.3.1 Homogeneous snow - allowable bearing capacity . . . . . . . . . 178

8.4 Summary . . . . . . . . . . . . . . . . . . . . . . . . . . . . . . . 179

9 Conclusion $\quad 181$

9.1 Main aims . . . . . . . . . . . . . . . . . . . . 181

9.2 Additional original contributions . . . . . . . . . . . . . 183

9.3 Limitations of this work . . . . . . . . . . . . . . . . . . 183

9.4 Further Research . . . . . . . . . . . . . . . . . . . . . . . 184

9.5 Conclusion . . . . . . . . . . . . . . . . . . 185

$\begin{array}{ll}\text { Bibliography } & 187\end{array}$

A Summary table of Antarctic CPT tests 203

B Standard Operating Procedures: Antarctic CPT 210

C Calculation of maximum penetrative force via Johnson's (2003) statistical micromechanical method 216 


\section{List of Figures}

Review 5

2.1 Sintering of ice grains. . . . . . . . . . . . . . . . . . 6

2.2 Evolution of snow microstructure over time. . . . . . . . . . . . . 8

2.3 Lower density snow may be stronger owing to microstructure. . . . . . 9

2.4 Variation of snow strength with density. . . . . . . . . . . . . . . 9

2.5 Decreased strength at higher deformation rates. . . . . . . . . . . . . 11

2.6 Methods of determining snow strength. . . . . . . . . . . . . . 17

2.7 Classical creep curve. . . . . . . . . . . . . . . . . . . . . . 19

$\begin{array}{ll}\text { Methods } & 28\end{array}$

3.1 Test location, Greenland. . . . . . . . . . . . . . . . . . . . . . . . 30

3.2 Greenland test sites. . . . . . . . . . . . . . . . . . . . 30

3.3 Fugro mini snow penetrometer. . . . . . . . . . . . . . . 31

3.4 Fugro mini-penetrometer friction sleeve. . . . . . . . . . . . . . . . 31

3.5 Main testing locations. . . . . . . . . . . . . . . . . . . . 33

3.6 UK11. . . . . . . . . . . . . . . . . . . . . . . . . . . 34

3.7 Basement rams. . . . . . . . . . . . . . . . . . . . . . 35

3.8 UK11 fitted to BAS tractor. . . . . . . . . . . . . . . . . . . 37

3.9 Zero-shift of cone tip resistance. . . . . . . . . . . . . . . . . . . 39

3.10 Thin hard layers hampered sample extraction. . . . . . . . . . . . . . . 42

3.11 Confined compressive strength apparatus. . . . . . . . . . . . . 44

3.12 Shear frame. . . . . . . . . . . . . . . . . . . 45

3.13 Tensile cell. . . . . . . . . . . . . . . . . . . . . . . 46

3.14 Typical shear test set-up. . . . . . . . . . . . . . . . . . 47

3.15 Opportunistic pressure bulb testing. . . . . . . . . . . . . . . . . 48

3.16 Pressure testing setup. . . . . . . . . . . . . . . . . . . . . . . 49

3.17 Pressure testing schematic. . . . . . . . . . . . . . . . . 49

3.18 Pressure sensor calibration curve. . . . . . . . . . . . . . . . . 50

3.19 Setup for investigating pressure-bulb beneath cone or flat plate. . . . . 51

3.20 Typical method of deploying the GPR . . . . . . . . . . . . . . . . 53 
Results \& Analysis (A) - Fugro mini-penetrometer $\quad 56$

4.1 Resistance approaches steady-state. . . . . . . . . . . . . . . 57

4.2 Steady-state resistance is obtained earlier in lower density snow. . . . . 58

4.3 Spacing of Tests 1 to $9 \ldots \ldots \ldots \ldots$. . . . . . . . . . . 58

4.4 Spatial consistency of tip resistance. . . . . . . . . . . . . . . . . . . . 59

4.5 Spatial consistency of sleeve friction. . . . . . . . . . . . . . . 59

4.6 Increase in tip resistance in older snow. . . . . . . . . . . . . . . . 61

4.7 Tip resistance is greater in denser snow. . . . . . . . . . . . . . . 62

4.8 Tip resistance increase due to icy layer. . . . . . . . . . . . . . . . . 62

4.9 Sleeve friction trends with tip resistance. . . . . . . . . . . . . 63

4.10 Horizontal testing method. . . . . . . . . . . . . . . . . . . 65

4.11 No compacted zone evident ahead of penetrometer. . . . . . . . . . . 65

4.12 Plug of snow ahead of unconfined penetrometer. . . . . . . . . . . 66

4.13 Chilli flakes used to show lack of compacted zone. . . . . . . . . . . . 67

4.14 Chipping during unconfined penetration. . . . . . . . . . . . . . 67

4.15 Chipping curve is similar to that for dense rock. . . . . . . . . . . . . 68

Results \& Analysis (B) - CPT $\quad 69$

5.1 Screen shot of typical CPT data. . . . . . . . . . . . . 70

5.2 CPT data from test pit \#1. . . . . . . . . . . . . . . . 70

5.3 CPT data from test pit \#2. . . . . . . . . . . . . . . . . . . . . . 71

5.4 Sawtooth resistance trace. . . . . . . . . . . . . . . . . . 72

5.5 Most variation in tip-resistance is owing to low frequency events. . . . . 72

5.6 Stick-slip behaviour in CPT trace. . . . . . . . . . . . . . . . . . 73

5.7 Positive correlation exists between sleeve friction and tip resistance. . . 74

5.8 Anomalous pore pressure reading observed post sea-ice testing. . . . . . 75

5.9 Schematic of cone pore-pressure measuring system. . . . . . . . . . 75

5.10 Estimated insitu vertical stress at snow pit \#1. . . . . . . . . . . . . . 77

5.11 Resistance horizons are spatially continuous. . . . . . . . . . . . . 78

5.12 Consistent horizons are evident from GPR data. . . . . . . . . . . . . . 79

5.13 Halley snow surface shows limited heterogeneity. . . . . . . . . . . . . 79

5.14 Qualitative resistance comparison. . . . . . . . . . . . . . 81

5.15 Variation of tip resistance with penetration rate. . . . . . . . . . . . 82

5.16 Variation in mean sleeve friction with penetration rate. . . . . . . . . 83

5.17 Variation in mean sleeve friction with penetration rate - 2. . . . . . . 83

5.18 Normalised resistance decreases as penetrometer size increases. . . . . . 88

5.19 Flat plate realises significantly greater resistance than the cone. . . . . 89

5.20 Mass of compacted snow forms on the flat plate as the test progresses. . 91

5.21 Screen shot of standard cone slowly advancing in cut-away hole. . . . . 92

5.22 Annulus of compacted snow after test. . . . . . . . . . . . . . . . 93 
5.23 Annulus of compacted snow after test - 2. . . . . . . . . . . . . . 93

5.24 Low-angle conical plug routinely observed on flat-plate post-test. . . . . 94

5.25 Friction decreases when compaction is reduced. . . . . . . . . . . 96

5.26 No 'sensing' is apparent ahead of the cone. . . . . . . . . . . . . . . . . 97

5.27 Decreased elastic resistance is mobilised at distance ahead of the cone. . 98

5.28 Method for removal of overburden. . . . . . . . . . . . . . . . . 99

5.29 Inconclusive variation of tip resistance with overburden. . . . . . . . . . 100

5.30 Variation of mean sleeve friction overburden. . . . . . . . . . . . . . . 101

5.31 Dissipation tests. . . . . . . . . . . . . . . . . . 103

5.32 Soil classification from CPT. . . . . . . . . . . . . . . . . 105

5.33 Friction ratio varies erratically with depth. . . . . . . . . . . . . 108

5.34 Scatter plot of tip resistance and sleeve friction. . . . . . . . . . . . . 108

Results \& Analysis (C) - Supplementary subsurface investigations $\quad 110$

6.1 Snow Pit \# 1 data. . . . . . . . . . . . . . . . . . . . . . . . . . 111

6.2 Snow Pit \# 2 data. . . . . . . . . . . . . . . . . . . . . . . 111

6.3 Confined compression strength testing data. . . . . . . . . . . . . . . . 113

6.4 Abele's (1970) load-sinkage relationship. . . . . . . . . . . . . . . . . . 113

6.5 Possible form of expected pressure-sinkage relationship. . . . . . . . . . 114

6.6 Failure resistance versus density. . . . . . . . . . . . . . . . . . . 115

6.7 Comparison of compressive strength data. . . . . . . . . . . . . . 116

6.8 Shear strength vs density. . . . . . . . . . . . . . . . . . . . . 118

6.9 John Deere 7820 tracked-tractor used in pressure bulb tests. . . . . . . 119

6.10 Pressure variation under John Deere tractor - 29 Dec 09. . . . . . . . . 120

6.11 Pressure variation under John Deere tractor - 10 Feb 10. . . . . . . . . 120

6.12 Comparison of vertical stress distribution. . . . . . . . . . . . . . 121

6.13 Tip resistance and GPR trace - 1. . . . . . . . . . . . . . . 123

6.14 Tip resistance and GPR trace - 2. . . . . . . . . . . . . . . . . . . 124

6.15 Tip resistance \& GPR amplitude. . . . . . . . . . . . . . . . . . . . . . 124

\section{$\begin{array}{ll}\text { Stratigraphy \& strength from CPT } & 126\end{array}$}

7.1 Determination of layer boundaries. . . . . . . . . . . . . 127

7.2 Determination of layer boundaries - 2. . . . . . . . . . . . 128

7.3 Comparison of generated and observed stratigraphy. . . . . . . . . . . . 129

7.4 Tip resistance and sleeve friction versus depth. . . . . . . . . . . . . 130

7.5 Tip resistance and sleeve friction superimposed over stratigraphy. . . . 131

7.6 Variation of tip resistance and density with depth. . . . . . . . . . . . 133

7.7 Normalised sleeve friction and density versus depth. . . . . . . . . . . . 133

7.8 Increased normal force in higher density snow. . . . . . . . . . . . . . 135

7.9 Comparison of tip resistance with quantified hand hardness. . . . . . . 136 
7.10 Exponential relationship between tip resistance \& Ramm number. . . . 137

7.11 Strain path for fractured particles moving ahead of cone. . . . . . . . . 143

7.12 Pressure and density data from Mellor (1975) . . . . . . . . . . . . . 148

7.13 Tip resistance and Ramm hardness versus snow shear strength. . . . . 154

7.14 Comparison of strength from CPT with compressive strength. . . . . . 155

\section{$\begin{array}{lr}\text { Application of CPT data } & 159\end{array}$}

8.1 Shear failure surfaces. . . . . . . . . . . . . . . . . . 163

8.2 Effective modulus derived from CPT. . . . . . . . . . . . . . 168

8.3 Elastic modulus is strain-rate dependent. . . . . . . . . . . . . . . . . . 169

8.4 Comparison of applied stress with estimated snow strength. . . . . . . . 175

8.5 Comparison of applied stress with estimated snow strength - II. . . . . 175

8.6 Comparison of applied stress with estimated snow strength - III. . . . . 176

\section{Appendix C}

C.1 Expression for maximum cone penetration force via statistical micromechanical reasoning. . . . . . . . . . . . . . . . . 220 


\section{List of Tables}

Review 5

2.1 Behaviour of snow of different density at different strain rates. . . . . . 12

2.2 Summary of snow penetrative instruments. . . . . . . . . . . . 26

$\begin{array}{lr}\text { Methods } & 28\end{array}$

3.1 Fugro mini snow penetrometer specifications. . . . . . . . . . . . . 31

3.2 GeoPoint 'Antarctic' cone specifications. . . . . . . . . . . . . . . . . . 36

3.3 GSSI TerraSIRch SIR System-3000 specifications. . . . . . . . . . . . 53

$3.4400 \mathrm{MHz}$ Antennae specifications. . . . . . . . . . . . . . . . . . 53

3.5 Typical SIR-3000 Operating Parameters. . . . . . . . . . . . . . . 54

Results \& Analysis (A) - Fugro mini-penetrometer 56

4.1 Fugro mini penetrometer testing. . . . . . . . . . . . . . . . 56

4.2 Variation in tip resistance and sleeve friction with penetration rate. . . 60

4.3 Variation of mean sleeve friction with density. . . . . . . . . . . . 60

4.4 Variation in mean normalised sleeve friction with density. . . . . . . . 63

Results \& Analysis (B) - CPT $\quad 69$

5.1 Basic Statistics - Tests $9,10 \& 12 \quad \ldots \ldots \ldots$. . . . . . . . . . 78

5.2 Dissipation Test Data . . . . . . . . . . . . . . . . . . 103

5.3 Friction Ratio variation between similar layers. . . . . . . . . . . . . 109

Results \& Analysis (C) - Supplementary subsurface investigations $\quad 110$

6.1 Resistance under confined compression versus density. . . . . . . . . . . . 114

6.2 Shear strength $(\mathrm{kPa})$ versus density - 11th Feb 2010 . . . . . . . . . 116

6.3 Shear strength $(\mathrm{kPa})$ versus density - 12th Feb 2010. . . . . . . . . . 117

6.4 Pressure vs Depth. . . . . . . . . . . . . . . . 120

Stratigraphy \& strength from CPT $\quad \mathbf{1 2 6}$

7.1 Resistance comparison - hand hardness and CPT tip resistance. . . . . 136 
7.2 Force comparison - Hand Hardness, Rammsonde and CPT Tip Resistance.137

7.3 Derivation of 'lock-up' density for hypothetical initial density and radii values of a general particulate material. . . . . . . . . . . . . . . 147

7.4 Increase in stress required to compact snow from initial to final density. 148

7.5 Variation of linear strain with initial density at constant stress (derived from $($ Abele, 1970)). . . . . . . . . . . . . . . . . . . . . . . . 149

7.6 Stress multiplier for snow of different initial densities. . . . . . . . . . . 149

7.7 Comparison - average shear strengths and average tip resistance - Halley Vehicle Park. . . . . . . . . . . . . . . . . . . . . 156

\section{Application of CPT data}

8.1 Hypothetical layer parameters for consideration of ultimate bearing capacity in layered snow; assumed average snow density of $450 \mathrm{~kg} \mathrm{~m}^{-3}$. .171

8.2 Summary of multi-layer elastic bearing capacity calculations. . . . . . . 172

8.3 Comparison of bearing capacity estimates in sand from CPT via direct methods. . . . . . . . . . . . . . . . . . . 178

8.4 Summary of estimated surface bearing capacity values expressed as percentage of mean CPT tip resistance. . . . . . . . . . . . . . . . . . . 180

\section{Appendix C}

C.1 Values used in Johnson's physical expression for the maximum cone penetration force. . . . . . . . . . . . . . . . . 220 


\section{Chapter 1}

\section{Introduction}

The Cone Penetration Test (CPT) is an efficacious process that may provide a rapid measure of strength, microstructure and density in polar snow. This thesis describes my research into the use of this procedure, where: "A cone on the end of a series of rods is pushed into the ground at a constant rate and continuous or intermittent measurements are made of the resistance to penetration of the cone" (Lunne et al., 1997). It is a unique study that examines the first use of a large diameter, hydraulically-driven cone penetrometer in polar snow.

\subsection{Motivation}

Investigation into the lifetime of the Wilkins compressed-snow runway near Casey Base, Antarctica (McCallum, 2006) prompted my interest in the siting and development of runways in Antarctica.

Although aviation has existed in Antarctica since the early 1900s, only recently has it been considered as a possible replacement for traditional ship-borne logistics. Permanent Antarctic stations require significant volumes of large cargo that are necessarily supplied by sea. However, large numbers of personnel and lighter equipment can more efficiently be transported by air. The development of air transport systems and supporting infrastructure, especially runways, is therefore assuming greater importance (Bergin and Haward, 2007; Fogarty, 2011).

Both blue ice and rock runway sites have been identified in Antarctica. However, the majority of the Antarctic continent is a snow accumulation area (Mellor, 1993) and methods to allow aircraft access to these areas is desirable. Ski-equipped aircraft for use on areas of snow have operated on the continent since the 1930s (Mellor, 1993) and continue to be used by most Antarctic operators today. However, the advent of retractable aircraft landing gear, and the added engineering complications and expense means that equipping a modern aircraft with skis is an expensive modification; retractable skis can be used, but the use of wheeled aircraft on suitable snow runways is preferable. Unfortunately, the majority of natural snow covered areas do not pro- 
vide a suitable surface for the operation of wheeled aircraft and the snow needs to be processed in order to obtain sufficient bearing capacity for wheeled aircraft.

Numerous authors have documented attempts by various nations to establish runways from processed snow (Mellor, 1993; Russell-Head and Budd, 1989), and techniques for building such runways have been practised for more than 50 years. When snow is disturbed by agitation or disaggregation, it begins to sinter and densify at a greater rate than undisturbed snow (Lee et al., 1988) and it is this behaviour that is exploited in the construction of compressed-snow roads and runways. The essential construction technique as summarised by Russell-Head and Budd (1989) is:

1. Process the snow to depth. This disaggregation is designed to reduce the size of the ice particles within the snow to aid in more rapid densification;

2. Compaction. This process further assists in the densification and strength-gaining process; and

3. Levelling. The pavement is levelled for vehicle use.

Essentially, the process to generate the required composition of snow for the construction of roads and runways is known. A combination of the above construction processes may be performed at different time intervals depending on the runway location and specific site characteristics including surface temperatures, accumulation, and snow pack initial conditions. This process was verified by Lang et al. (1997) who in examining the characteristics of processed snow concluded that the generation of a robust snow pavement is best expedited by producing a well-graded, small-grained and firmly compacted section of snow.

Design criteria for roads or runways of compressed snow typically specify the required snow characteristics via strength or similar indices. Rather than performing expensive processing of the snow pack to produce snow layers of suitable strength, a means of rapidly assessing the natural snow strength profile is desirable, so that favourable construction sites can be identified before the commencement of expensive snow processing.

My motivation for this research was therefore to devise a means whereby areas of natural polar snow may easily be tested for their suitability as compressed roads or runways, for use by wheeled vehicles. A natural extension of this is to extend the scope of application, such that an assessment of snow strength or bearing capacity can be used in the design and development of any load-bearing infrastructure in the polar regions, such as roads, runways and buildings or support structures.

\subsection{Purpose of my research}

The intent of my research was to investigate the use of CPT as a means of assessing the natural strength of polar snow, to enable optimum snow road and runway sites 
to be selected, thereby minimising the time and cost of processing excessive amounts of natural snow to achieve desired pavement design criteria. Specific questions that I address include:

1. Can a measure of snow strength be obtained via CPT, with or without the use of additional density data, and

2. Can the shallow bearing capacity of the snow be derived both directly and indirectly from CPT data, with or without additional density information?

My investigations into addressing these aims commenced with no financial support for field work, thus initial work focussed on desk-top analysis of the problem. During this initial phase I endeavoured to seek support, financial or in-kind via numerous government and corporate agencies, to enable suitable field-testing of my proposed investigative method. Eventually both financial and material support was provided through Gardline Geosciences, and additional support from the British Antarctic Survey (BAS) enabled access to the Halley V Research Station, Antarctica for a comprehensive season of field-testing, using equipment not previously employed in polar snow.

It is this field testing, plus some additional testing in Greenland, that is discussed within this thesis, and which provides the basis for addressing my aims above plus additional research questions, posed throughout.

\subsection{Significance of this thesis}

The work considered in this thesis is original because it describes the first use of a large diameter, hydraulically-driven cone penetrometer in polar snow; it describes the use of a penetrometer that measures both tip resistance and sleeve friction, in snow that would otherwise have been impenetrable, to otherwise unobtainable depths. This work discusses the development and employment of this tool and examines in detail the interpretation and use of that data. Original thought is then applied to the derivation of snow strength indices from the CPT and their application to the addressing of real engineering problems, such as the surface bearing capacity of a snow surface. Specifically the original contributions presented within this thesis include:

1. first use of a friction sleeve on two types of penetrating cone in polar snow,

2. estimation of snow layer strength directly from CPT data,

3. conceptualisation of the forces acting on both the cone and friction sleeve,

4. estimation of surface bearing capacity from CPT data, and

5. consideration of the similarities between snow and other geomaterials such as calcareous sands. 
An additional strength of this work is that it draws upon literature and advice from diverse fields such as soil mechanics, offshore geotechnical engineering, marine geosciences and agricultural engineering, in addition to snow mechanics and glaciology, and attempts to provide a 'non-blinkered' investigation into the topic.

\subsection{Thesis Outline}

In this thesis the broad context of the problem is considered before specific techniques and results are presented. These results are then examined and interpreted to address the objectives of this thesis, then engineering application of the CPT process is considered. This thesis proceeds as follows: Chapter 2 provides an introduction to the material snow, and how it might be expected to behave during penetrative investigations of strength. Chapter 3 outlines the methods I employed in conducting my testing, both CPT and supporting investigations, and also elaborates on some of the phenomena I was hoping to investigate. Chapters 4, 5 and 6 then present results and provide analysis of data collected during my research. Chapter 4 examines the Fugro minipenetrometer testing that was conducted as a pilot study in Greenland in 2009 to gain initial insight into penetrometer behaviour in polar snow. Chapter 5 deals with CPT in Antarctica in 2010, from which the primary conclusions of this thesis are drawn, and Chapter 6 deals with additional, sub-surface investigations that were conducted to assist in the interpretation and application of CPT data. Chapter 7 looks specifically at deriving stratigraphy and strength from CPT data; existing methods are examined, then an equation to derive snow strength from CPT resistance data is proposed. Chapter 8 provides some initial investigation into how strength indices obtained via CPT can be used to provide meaningful engineering data for the development of polar infrastructure, then Chapter 9 concludes this work, summarises my findings, places my work in context and reiterates the original contributions that I have made. I end by postulating on further work that could be conducted on this topic. 


\section{Chapter 2}

\section{Review}

The purpose of this review is to discuss the nature of snow: how it forms, typically behaves and thus how its behaviour under penetration might be interpreted; other analogues for snow are also considered in an effort to gain additional insight into its behaviour. Methods of assessing snow strength are then briefly examined before specific assessment via penetrative means is discussed; some matters pertaining specifically to the CPT are then introduced. Additional discussion occurs where appropriate and as necessary throughout this work.

\subsection{The Nature of Snow}

In general, snow on the ground can be described as a three-phase geomaterial consisting of ice (solid), pore water (liquid) and void air (gas). In this work on cold polar snow, it is assumed that snow is a two-phase material consisting of ice and air. Pielmeier and Schneebeli (2003) utilise a geological description, considering it an aeolian sediment with rapidly changing properties, that ultimately forms stratified layers via sedimentation.

Although the term firn is generally used for wetted snow that has lain on the ground throughout a summer and is in the process of being transformed to ice (Paterson, 1994), throughout this work I will use the term snow to describe the two-phase mixture of air and ice particles that comprises snow on the ground; firn will not be used.

\subsubsection{How does it form?}

As surface snow is buried by fresh snow on a glacier or ice sheet, it gradually compacts, eventually becoming ice. Throughout this process the snow is increasing in both density and strength. As soon as snowflakes hit the surface, the free energy of the system tends towards a minimum, such that dendritic and irregularly shaped crystals with a large surface area are gradually transformed to rounded particles (Paterson, 1994). This reduction in surface energy of the system is the primary driving force for the process 
of sintering. Sintering is defined as "a thermal treatment for bonding particles into a coherent, predominantly solid structure via mass transport events that often occur on the atomic scale" (Blackford, 2007). Because the radius of an ice particle is larger than the concave radius of the bond joining it to an adjacent ice particle, there is a driving force to move mass to this concave neck area. So two previously spherical particles start tending towards a 'dumb-bell' shaped particle joined by a neck, as shown in Figure 2.1.

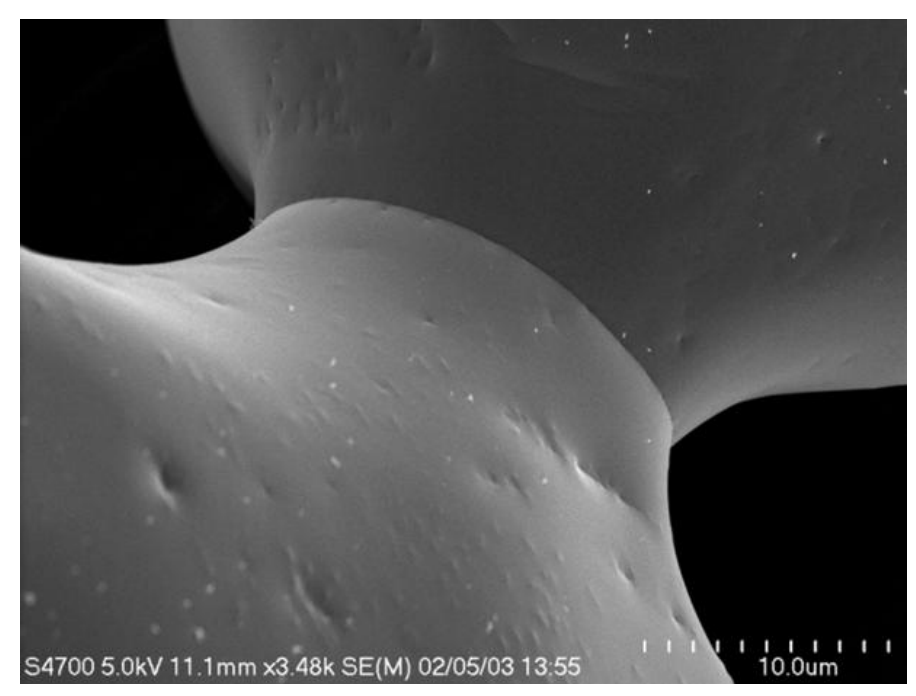

Figure 2.1: A low temperature SEM image of two ice grains, made by spraying water into liquid nitrogen, sintered at $-25^{\circ} \mathrm{C}$ for $216 \mathrm{~h}$. The grain boundary can be seen at the neck between the particles; the particles are single crystals. (from Blackford (2007)).

The bonding leads to improved strength and a lower energy system (German, 1996). This process of mass distribution occurs throughout the densification process. When this process occurs under additional pressure such as when snow is buried owing to accumulation, it is termed pressure sintering, and the rate of sintering is increased. Alley (1987) clarifies that although numerous processes occur throughout the pressure sintering process, not all contribute to densification. Three main phases have been identified in the densification process (Herron and Langway, 1980):

1. Stage 1. This process is essentially one of settling and packing whereby particles of ice are displaced relative to their neighbours. Whilst initially bonds may be broken, eventually upon close packing, stable bonds begin to form and grow. Alley (1987) determined grain boundary sliding to be the primary mechanism during this process. The average number of bonds between ice grains (coordination number $=\mathrm{N})$ has been observed to become constant $(\mathrm{N}=6)$ at a relative density of approximately 0.6 (Alley, 1987), corresponding to a density of approximately $550 \mathrm{~kg} \mathrm{~m}^{-3}$. After this, the size and strength of the bonds increase, without additional bonds being formed. (This is consistent with the typical close-packing density for spheres which pack to a density of $64 \%$, whereas recent work by Frenkel (2010) suggests that tetrahedrons (which ice crystals may approximate 
in shape) can pack to a tighter density of $\sim 76 \%$.) Colbeck (1998) notes that neck growth occurs rapidly initially, explaining why snow develops some strength quickly, with smaller grains sintering at a larger rate than larger grains. The strongest bonds and thus strength ultimately occur from small rounded grains, which grow at a slow rate.

2. Stage 2. The second stage of the process occurs from a density of $550 \mathrm{~kg} \mathrm{~m}^{-3}$ through to about $840 \mathrm{~kg} \mathrm{~m}^{-3}$, and involves a decrease in pore space within the snow, such that interconnected pore spaces become closed. This occurs through a process of plastic deformation via power law creep combined with recrystallisation (Blackford, 2007). Necks between grains continue to grow, however, the rate of growth decreases, and no new bonds are formed.

3. Stage 3. During the third stage, pores within the snow are closed and the snow is generally considered as an isothermal system; it may be described as bubbly (Blackford, 2007) or closed-cellular (Petrovic, 2003) ice. The density during this phase increases from about $840 \mathrm{~kg} \mathrm{~m}^{-3}$ through to the density of pore free ice at $-25^{\circ} \mathrm{C}, 919 \mathrm{~kg} \mathrm{~m}^{-3}$, and the densification proceeds primarily by the same mechanisms as stage 2 , but at a reduced rate. As the bubbles are compressed, this opposing pressure causes the process to slow.

The densification process progresses at a decreased rate in each stage, and Mellor (1975) says that in the natural environment it also occurs at different times throughout the year, commensurate with times of increased accumulation. This is supported by recent work by Arthern et al. (2010) who note strong seasonality in measured rates of snow compaction in Antarctica. The process of intermittent compaction appears similar in nature to the process of "self-organised criticality" noted by Sammonds (2005), where a natural system organises itself not at a constant macroscopically 'plastic' rate but in lurches as equilibrium is maintained within the system, on the microscale.

Throughout the densification process, the microstructure or the nature of the ice grains and the physical bonding between them is constantly evolving. This evolution is influenced by the initial thermal regime within the snow, and two distinct processes have historically been used to describe this effect on grain size down to a depth of approximately 2.5m (Alley, 1987).

Temperature gradient metamorphism (more recently kinetic growth (Fierz et al., 2009)) occurs when a strong temperature gradient exists and results in rapid nonlinear grain growth and a lack of cohesion. Over time this will result in coarse-grained layers with reduced density (Rick and Albert, 2004). This type of snow is often seen at depth as a seasonal layer of buried surface-hoar, which has formed during a previous surface summer season when temperatures were high. When a strong temperature gradient is absent, equitemperature metamorphism (more recently equilibrium growth 
(Fierz et al., 2009)) occurs. This results in surface layers experiencing slower, more linear grain growth, resulting in a finer grained layer of increased density.

In his study on the preferred method of producing compressed snow runways Abele (1990) identified that although temperature gradient metamorphism results in an increased rate of densification and an increase in short-term strength, it is equitemperature metamorphism that will ultimately produce a stronger pavement even though the rate of strength increase will be less. Thus although the initial strength of the snow may be less than rapidly sintered snow (nearer to $0^{\circ} \mathrm{C}$ ), the ultimate bond strength over time will be greater.

Perhaps the primary mechanical observation to be drawn from the above discussion is that the strength of snow is controlled by the size of the bonds between grains; the bonds formed between grains by sintering that generally grow with time giving strength to the bonded matrix of particles (Colbeck, 1998). This is the microstructure of the snow. Although strictly defined as the configuration of the ice and air spaces within the snow incorporating, density, porosity, specific surface area, curvature, tortuosity and coordination number (Fierz et al., 2009), I choose to define it more specifically as the size and number of bonds between ice grains within the snow. The evolution of microstructure over time can be seen in Figure 2.2.

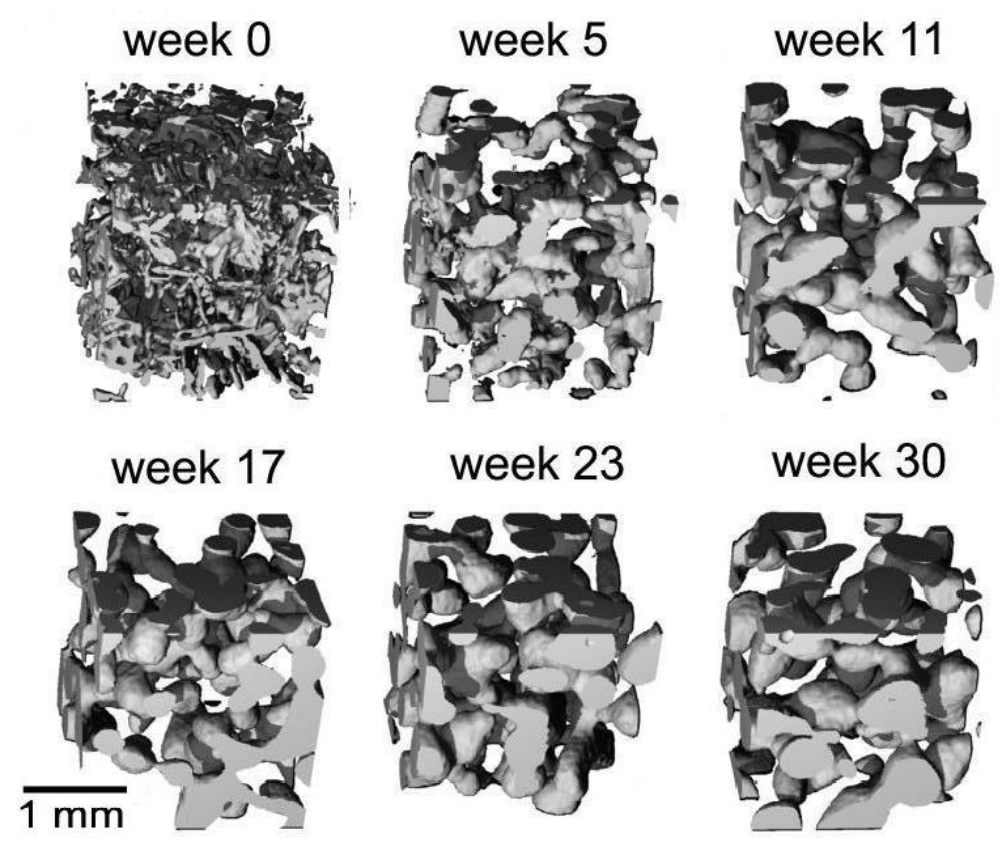

Figure 2.2: Evolution of the 3D structure of the ice matrix during isothermal metamorphism at $-1.6{ }^{\circ} \mathrm{C}$ over a 30 week period. Adapted from Kaempfer and Schneebeli (2007).

It is the strength provided by this bonded microstructural matrix and not solely the density that will account for snow's mechanical behaviour. Therefore, any attempt to understand the mechanical behaviour of snow, such as whilst it is subjected to CPT, must consider both the bulk density and the microstructure of the snow. As illustrated in Figure 2.3 and Figure 2.4, snow of a lesser density may be stronger than 
a less well-bonded snow of higher density. The stylised variation suggested in Figure 2.4 is consistent with the range of strength exhibited by natural snow at a particular density as presented by numerous authors, particularly Mellor (1975), who shows that order of magnitude differences are observed.
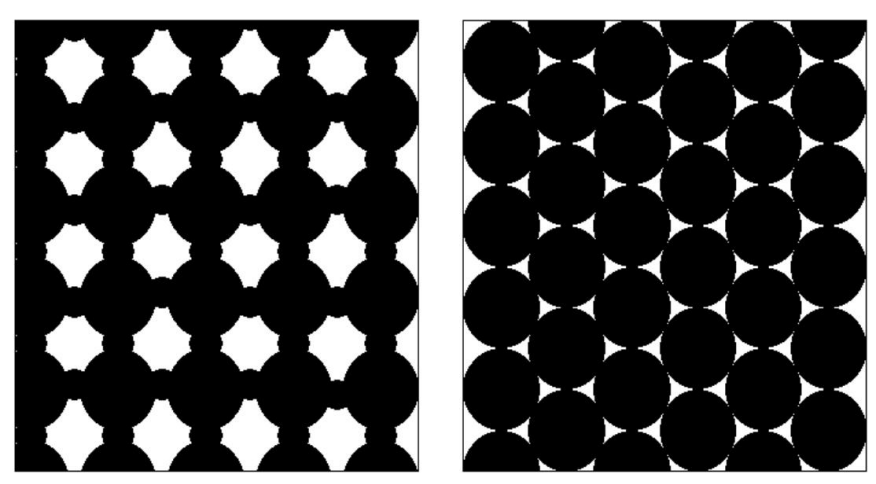

Figure 2.3: Sample on the left has the least density, but will be stronger because of bonding between grains, whereas the sample on the right is of higher density but will be weaker, owing to negligible bonding between grains.

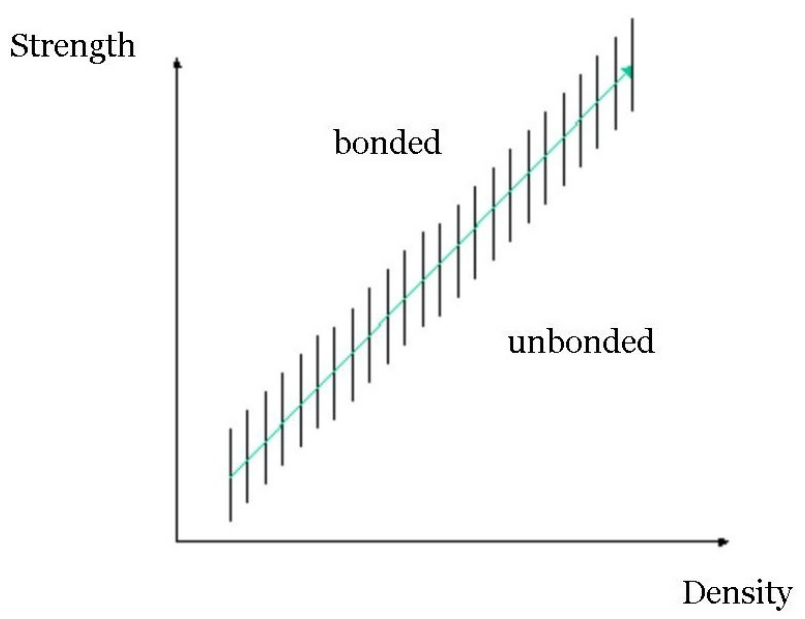

Figure 2.4: Stylised depiction of snow strength generally increasing with density; higher density snow may have lower strength because of variations in microstructure.

The behaviour noted above for snow is very similar to the description of soil behaviour given by Schofield (1998): "When soil flows, many soil particles change partners, and there is no time to bond particle to particle. It is only when soil is left to age and creep that bonds develop at particle contacts and turn it into soft rock." Structure has been shown to define mechanical behaviour across geomaterials (Leroueil and Vaughan, 1990) and although modern snow research has progressed from its initial historical reliance on ideas taken from soil mechanics, comparison of snow mechanical behaviour with that of soil (from which most CPT experience has been derived) and other geomaterials remains a useful technique and forms an important part of this work. Further possible analogues for snow are briefly discussed in subsection 2.1.3. 


\subsubsection{How does snow behave?}

Snow possesses strength due to the number and size of bonds between grains. This microstructure also determines the rheology of snow. At the extreme limits of a continuum, snow can be considered either completely unbonded, or completely bonded. Under low stresses and low strain rates it may therefore flow as a particulate or granular material, or it may deform elastically then flow as a viscous fluid. Low density snow is also highly and irreversibly compressible. Mellor (1975) describes snow as a compressible non-linear viscoelastic material, exhibiting both viscous flow (as a fluid) and elastic behaviour (as a solid). As snow approaches the density of ice it assumes the behaviour of a solid and the term plastic may be used to describe its deformation, consistent with Petrovic's description of snow as a cellular form of ice (Petrovic, 2003).

As implied above, snow also behaves differently depending on the rate at which it is loaded. Extremes in loading may vary from the gradual loading of snow owing to accumulation through to rapid loading via a skier or a vehicle. Snow behaves differently under each of these scenarios and various attempts have been made to classify this behaviour. Whilst the rheological behaviour of dense snow or ice when subjected to low strain rates can be described by the process of creep, very different behaviour is exhibited by snow experiencing high rates of strain.

When snow of high density is subjected to high strain rates, it behaves in a manner similar to ice, and will generally fail in a brittle manner either by fracture of bonds between the grains or fracture of the grains themselves (Scapozza and Bartelt, 2003b). This observation is supported by Fukue (1977) who stated that snow subjected to high deformation rates fails in a brittle manner because of grain breakage or intergranular slippage, to the exclusion of ductile behaviour. Yosida (1963) proposed a critical deformation rate or critical velocity which defines the transition between the ductile and brittle behaviour.

For snow of a given low density subjected to high strain rates, there can be large variations in behaviour (for snow of the same density) (Haehnel et al., 2004), and typically there is large volumetric straining owing to the compressibility of the snow. Once volumetric deformation and close-packing has been achieved, the snow may then work harden and deform plastically under any additional external stresses, offering increased resistance to any imposed stress. Scapozza and Bartelt (2003b) found that work hardening commences at about the same strain regardless of the rate of deformation.

As an example, Figure 2.5 shows the variation in unconfined compressive strength between the regimes of ductile and brittle behaviour and the critical deformation rate dividing these zones. Also shown is the increase in compressive strength as snow is allowed to sinter for increasing periods of time.

The behaviour illustrated in Figure 2.5 is similar to that of ice which also shows an increase in compressive strength through the ductile zone until the brittle/ductile transition, before a reduction in compressive strength is observed as strain rate increases 


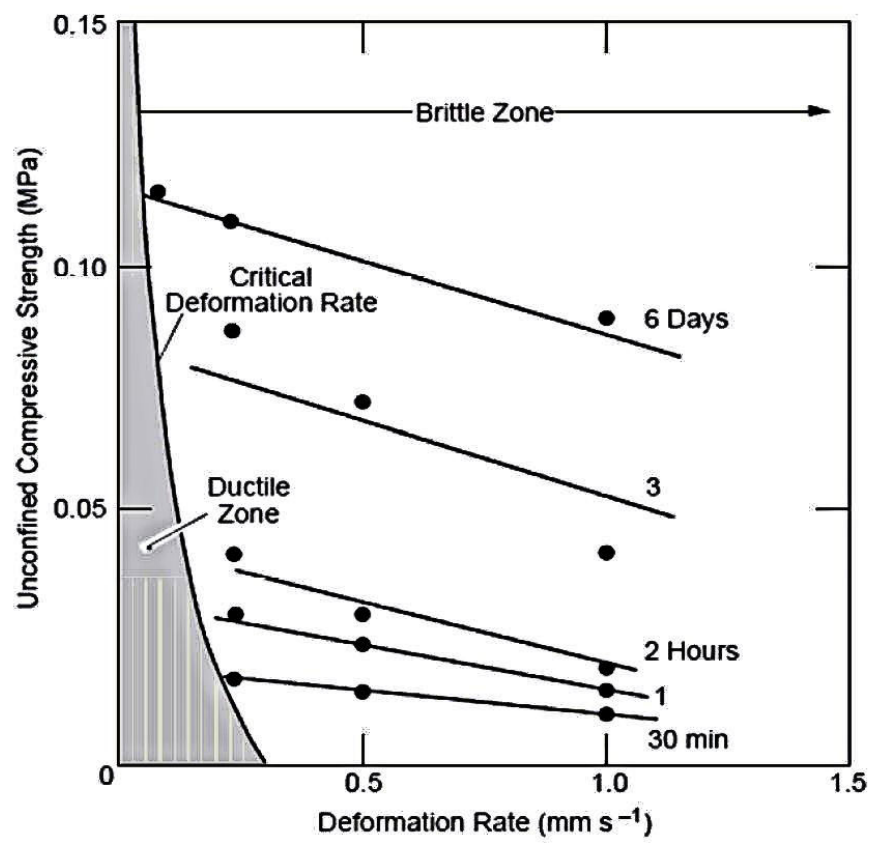

Figure 2.5: Experimental data from Fukue (1977) shows that the unconfined compressive strength for snow is less at higher rates of deformation and that increased sintering with time results in increased strength.

within the brittle zone (Schulson, 2001). The brittle/ductile transition is not a point, but rather a zone where the snow exhibits semi-brittle or semi-ductile behaviour. Fukue (1977) notes that the critical deformation rate at which transition from ductile to brittle behaviour occurs is $\sim 0.1$ to $0.2 \mathrm{~mm} \mathrm{~s}^{-1}$; Gardea et al. (1996) say the transition occurs at $\sim 10 \mathrm{~mm} \mathrm{~min}^{-1}\left(\sim 0.16 \mathrm{~mm} \mathrm{~s}^{-1}\right)$, Kronholm (2004) suggests that brittle failure in snow is expected at strain rates greater than $10^{-3} \mathrm{~s}^{-1}$, whilst Floyer and Jamieson (2010) say $\sim 1 \times 10^{-3}$ to $\sim 1 \times 10^{-4} \mathrm{~s}^{-1}$ depending on the snow type.

The decrease in compressive strength as strain rate increases in the brittle zone appears established for frozen geomaterials, for example, work by Ladanyi (Ladanyi and Paquin (1978) and Ladanyi (1981)) on frozen sand showed increasing strength up until the brittle zone, whereupon strength then decreased with rate. However, contrary to this behaviour Yuanlin et al. (1998) on tests of frozen silt in compression do not report a decrease in strength with increased rate once in the brittle zone, although equipment limitations limited their testing rate. Although variations will likely occur with the ice content of the soil, snow's behaviour appears consistent with other frozen geomaterials.

The behaviour of snow of varying densities subjected to different strain rates is summarised in Table 2.1.

The reason for the transition in behaviour across the ductile - brittle zone has been considered by numerous authors including Kartashov (1965) and Schulson (2001) and is supposedly because of competition between two processes: the weakening of the snow because of the fracture of structural connections, and the strengthening of the snow 
Table 2.1: Behaviour of snow of different density at different strain rates.

$\begin{array}{ll}\text { Snow Density } & \text { Low Strain Rate } \\ \text { Low } & \text { Compresses with volumet- } \\ & \text { ric creep resulting in higher } \\ & \text { density. }\end{array}$

High Deforms in a macroscopically ductile manner eventually reaching failure via tertiary creep (behaviour similar to ice).

\section{High Strain Rate}

Initially brittle failure (after small elastic deformation) via bond fracture or grain fracture then interparticle slip and large volumetric straining leads to rapid densification. Work hardening may result.

Brittle failure (after small elastic deformation) via bond fracture or grain fracture.

owing to compaction and dislocation slip. At the transition rate of strain the ice can no longer relieve stress sufficiently via ductile processes, and brittle fracture results.

So, the behaviour of snow depends on the nature of the snow itself and the nature of loading. Both of these factors need to be considered in assessing the behaviour of snow when subjected to cone penetration testing. This is discussed further shortly, but firstly it is necessary to consider an analogue that will adequately describe the behaviour of medium density snow at the high strain rates experienced during CPT.

It is assumed that immediately post-CPT, the snow will have been fractured, and will be in a particulate state such that its behaviour can be considered that of a frictional granular material. The subsection below therefore considers whether use of the Mohr-Coulomb model is appropriate to describe the behaviour of this post-fracture frictional material.

\subsubsection{Mohr-Coulomb model}

The Mohr-Coulomb criterion describes the relation between the shear strength of a material and the applied normal stress. It is expressed as:

$$
\tau=C+N \tan \phi
$$

where $\tau$ is shear strength, $\mathrm{C}$ is cohesion, $\mathrm{N}$ is applied normal stress and $\phi$ is the friction angle of the material.

Mellor (1975) states that the idea of treating snow as a Mohr-Coulomb cohesive frictional material is both attractive and historically practised. McClung and Schaerer (1993) and Chiaia and Frigo (2009) (in their assessment of snow slab avalanches) treat snow shear strength as a function of cohesion and friction and Fellin and Lackinger (2007) describe temperate glacier ice as a purely cohesive material. Across the field of 
cryospheric research, the Mohr-Coulomb model has been widely used.

Mellor (1975) questions "whether internal friction can be fully mobilized until C is effectively destroyed", and therefore suggests that snow (at least on the microscale) is possibly best described as a cohesive or frictional material rather than a cohesive and frictional material. This is consistent with Martin (1997) who says that the cohesive strength predominates any mobilised frictional strength, and is later replaced by the frictional strength when cohesion is lost. It should be noted that in considering bulk snow it is likely that on the macroscopic level some level of both frictional and cohesive behaviour may be exhibited, even though on the microscale only one of these behaviours is likely mobilised.

Following Mellor (1975), I propose to apply the Mohr-Coulomb criteria in the following manner:

1. before bond breakage the snow has cohesion, but no friction (as no inter-particle movement has occurred), and

2. after bond breakage, the snow has friction but no cohesion.

So the shear strength of the snow is assumed equal to the cohesion of the snow prior to failure, and post-failure the shear strength (assuming no immediate resintering of particles) is considered a function of the friction between fractured particles and the normal stress.

\subsubsection{Other analogues for snow}

It has already been suggested that snow may broadly be considered a geomaterial and that similarities in structure (or microstructure, the bonding between particles) between such materials may define similar mechanical behaviour. Although the behaviour of snow may be unique in many ways, it is also very similar to a vast range of man-made and natural materials, owing to its structural form at certain densities, and such comparison may provide some insight into the behaviour of snow whilst undergoing CPT. Analogues that could be used to describe snow's behaviour under load are now briefly considered:

Geomaterials. Leroueil and Vaughan (1990) in their review of the structure of weak rocks and natural soils showed that deposits normally treated as 'soils' usually have "characteristics due to bonded structure which are similar to those of porous weak rock", resulting in mechanically stiff behaviour followed by yield. They show that such characteristics are common in natural geological materials and that it is the structure of weak rock and cemented sands that gives them their strength. This "structure, arising from different causes, gives similar behaviour in many different materials" including snow. Leroueil and Vaughan (1990) also suggest that geomaterials generally share other 
behavioural similarities such as a strain-rate dependent strength, similar to behaviour observed within snow and ice.

Sedimentary material. Snow has historically been viewed specifically as a sedimentary material (Benson (1962), Pielmeier and Schneebeli (2003) and Schweizer et al. (2008b) amongst others) that ultimately may develop structure and thus behave in accordance with Leroueil and Vaughan's supposition (Leroueil and Vaughan, 1990) above.

Porous Rock. Leite and Ferland (2001) in their work on the indentation of porous material note linear elasticity, yielding and structural collapse at a critical value, and strain hardening as the crushed material is compacted; all these behaviours are observed in the penetration of snow (of certain density). Tharp (1983) in his work on polyphase rocks suggests that "incompetent phases" (essentially non-load-bearing phases such as air) within a material result in the load-carrying framework behaving much like a porous solid, and snow has been considered as such by numerous authors including Brown (1979), Kirchner et al. (2001), and Petrovic (2003).

Foam. Snow has been specifically considered as a foam or a cellular solid by Brown (1979), Kirchner et al. (2001), and Petrovic (2003) and others including Johnson (2003). In his work on a statistical micromechanical theory for penetration in granular materials Johnson (2003) draws upon initial work presented by Gibson and Ashby (1997) who examine the behaviour of foam under penetration in their review of the behaviour of cellular solids. Johnson's work, including that with Schneebeli (Johnson and Schneebeli, 1999) provides most of the basis for the means of interpreting data from the Snow Micro-Penetrometer (SMPT), a penetrometer used successfully and extensively in snow research, further described in a subsequent section. Examination of Gibson and Ashby's work (Gibson and Ashby, 1997) suggests that below the pore close-off density of $\sim 840 \mathrm{~kg} \mathrm{~m}^{-3}$, snow can be regarded as an open-cellular foam, whilst above this density, description as a closed-cellular foam may be appropriate. However, verbal discussion with M. Ashby (17th December 2008) suggests that caution is necessary in adopting such a description as the heterogeneous nature of snow and the variation in behaviour as both density and structure change may prohibit accurate comparison with foam. Foam has previously been used as an analogue for snow and similarities in behaviour may exist.

Polycrystalline ice. The main constituent of dry snow is ice; snow's behaviour must tend towards that of polycrystalline ice as density increases. Nicot (2004) states that on the microscopic scale, the behaviour of grain bonds (within snow) is governed by the behaviour of ice, Gubler (1978) describes the load-bearing capacity of snow in terms of ice "chains", and Bartelt and von Moos (2000) note the straining of the ice lattice within snow during triaxial testing. Graphs of stress versus strain rate generated by Kinosita (1967) through loading of snow are almost identical in form to those presented by Schulson (2001) for polycrystalline ice. Although there are differences in scale (snow on the macroscopic level will collapse and compact under loading), the linking of the 
microstructural behaviour of snow with that of ice appears appropriate.

Sintered metal. Finally, snow can be considered as a sintered material such as a metal where under stress "the usual fracture of the sintered necks takes place, as for most porous material" (Tancret and Osterstock, 2003). This behaviour is consistent with the failure mechanism for snow described by Mellor (1966) outlined earlier.

The similarities between snow and some other natural and man-made materials have been briefly discussed, and the expected behaviour of snow under penetration is now considered.

\subsubsection{Behaviour during penetration}

Substantial research has been conducted into the behaviour of snow whilst loaded under flat plates, see Wuori (1962), Abele (1970), Russell-Head and Budd (1989) etc. Such testing typically measures settlement (or plate penetration) as a function of pressure for snow of various densities and has almost become the standard field test for snow mechanical testing purposes.

Such testing reveals the following characteristics of snow whilst undergoing penetration via a flat plate:

1. an initial often immeasurable elastic deformation occurs, before

2. compaction occurs commensurate with snow density; the higher the initial density the less the compaction;

3. higher density snow provides greater resistance,

4. plate size affects penetration distance,

5. collapse occurs in a step-like discontinuous manner,

6. penetration distance depends on penetration rates, and

7. repeated penetration (particularly of initially low-density snow) may result in all intergranular bonds within the snow being destroyed so that the snow reverts to a fluidised granular material that is unable to resist shear (Abele, 1970).

Although not all of the phenomena described above may occur during the continuous penetration of snow via a conical object (such as in CPT), some of them may. During CPT the following might be expected (assuming strain rates are within the brittle zone):

1. initial elastic deformation,

2. brittle fracture of individual grain bonds will occur at the brittle strength of the ice, 
3. fractured particles will be displaced normally to the cone face, resulting in a 'compacted zone' (Johnson, 2003) ahead of and to the side of the cone,

4. material beyond this compacted area will experience elastic deformation, possibly out to a radius of 2 to 3 times the cone diameter,

5. the compacted zone will evolve throughout a test and may approach a steady state (possible in a homogeneous material), however, changes in layer strength and bonding will continually affect its dimensions,

6. elastic deformation of particles ahead of the cone (or possibly engaged via the inert compacted zone ahead of the cone) may contribute to measured force,

7. failure may appear continuous on a macro-level, but is likely discontinuous on the micro level as successive fracturing of bonds takes place,

8. cone shape will affect measured stress,

9. cone size will affect measured stress, and

10. penetration rate will affect measured stress.

This is the assumed model of behaviour ahead of a penetrating cone in bonded snow that I will adopt in this work; rates of penetration will effect brittle rather than ductile failure. This model is largely consistent with penetration in cellular solids described by Gibson and Ashby (1997), with penetration in porous rock described by Leite and Ferland (2001), with penetration in granular materials described by Johnson (2003) (later applied directly to snow), and is consistent with the process described by J. Johnson, personal communication, 31 July 2009.

Points to note with respect to this expected behaviour are:

1. In the unlikely event that the snow is completely unbonded, resistance to cone penetration will come solely from friction between grains,

2. At densities greater than the close-packed density of natural snow $(\sim 550 \mathrm{~kg}$ $\mathrm{m}^{-3}$ ) grain fracture is likely, as further packing is not possible without breaking of snow grains, and

3. upon initial penetration near the surface, some mass expulsion will occur to this free boundary.

Thus my assumed model of expected behaviour needs to be applied cognisant of these limitations, and is probably only valid in bonded snow, at depth, up to a density of $\sim 550 \mathrm{~kg} \mathrm{~m}^{-3}$. Many of the mechanisms above are discussed at greater length in Chapter 7 . 
The section above has described the nature of snow, what it is composed of, how it is formed, and how it might broadly behave under different loading scenarios. Other analogues for snow have also been discussed to gain additional insight into snow's behaviour under penetrative loading, and finally the specific behaviour of snow under penetration has been considered. The next section will examine the typical methods of assessing snow strength, the interrelationships between these various indices, including some definitions, before specifically, penetrative testing in snow will be examined along with the history of Cone Penetration Testing. This will culminate in the conclusion that existing commercial CPT technology can be utilised to good effect for the assessment of both scientific and engineering parameters in polar snow.

\subsection{Strength testing in snow}

Abele (1990) says that although the strength of snow can be determined by various methods, there are three primary measurement techniques (Figure 2.6):

1. surface loading, in which a vertical load is applied to the snow surface, such as in a plate-bearing test,

2. sample strength testing, in which a sample is removed from the snow and subjected to a strength test, typically within a laboratory, and

3. probing, in which a penetrative device is forced into the snow obtaining a proxy for snow strength.

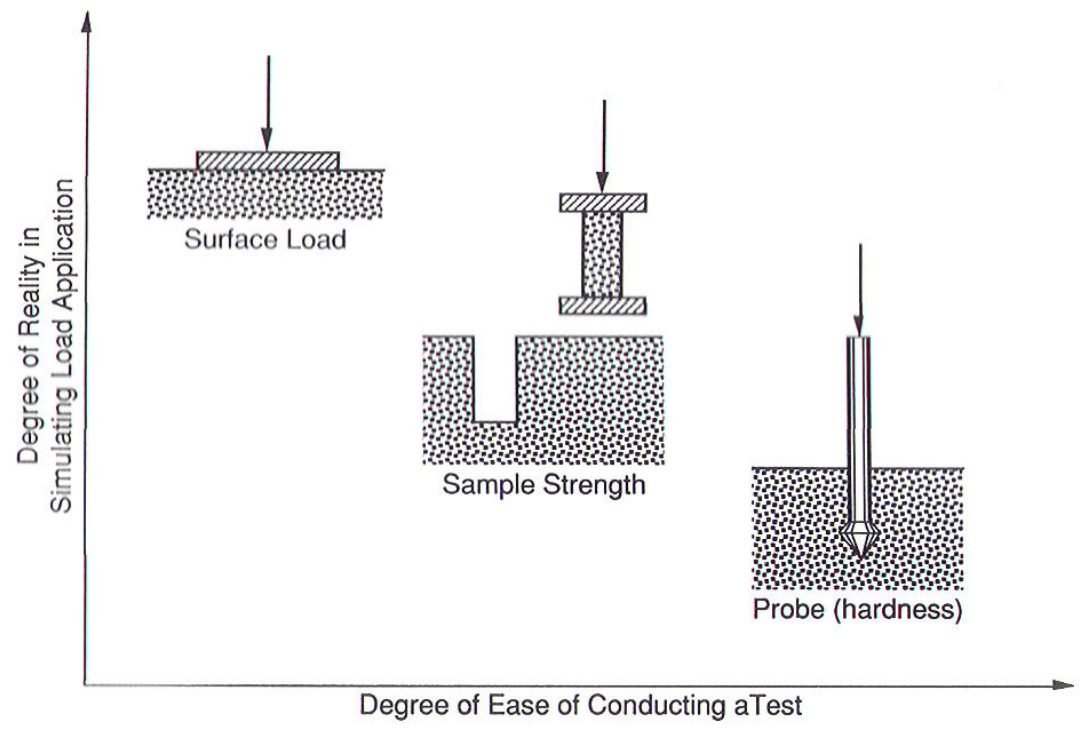

Figure 2.6: Three primary methods can be used to assess snow strength. The relative degree of reality in simulating load application varies with the relative ease of the test method (from Abele (1990).) 
Abele states that these can be considered more simply as "bearing tests, strength tests and hardness tests". These different tests determine slightly different facets of snow behaviour; the relationships between strength tests and probing tests are discussed shortly. Further discussion on surface loading and surface bearing capacity occurs in Chapter 8.

Mellor (1975) states "It may be surmised that, in most cases, the rupture of snow is governed by shear in the grain bonds, a supposition supported by correlation between shear, compression and tensile strengths." That is, although the strength of snow is described as compressive, tensile, shear, according to the nature of the test, the snow itself is likely failing primarily in shear, microscopically at a grain boundary or bond; the descriptor is merely describing the nature of testing undertaken in assessing this strength. This definition is consistent with Whitlow (2001) who states with respect to soil that "although strength can be stated in terms of compressive stress or tensile stress, fundamentally it's the ability to sustain shear stress that provides strength". Noting the above, it remains feasible for true compressive or tensile failure of a grain bond within snow to occur, depending on the nature of the snow and the loading scenario. Before these different strength indices are briefly examined and compared, the term failure needs to be better defined.

The failure of an engineering material is described by Mellor (1975) as being largely arbitrary; it refers to the condition when performance ceases to be satisfactory, and may involve rupture, failure of bearing-capacity, and excessive strain or strain-rate. Three general material failure types may be defined:

1. Elastic. The excessive elastic deflection of material in service, or the elastic instability of a material,

2. Ductile. Plastic deformation of the material such that the yield strength of the material is exceeded, or

3. Brittle. A pre-existing crack propagates rapidly through the material so that the material fails.

When designing foundations in snow, the overriding consideration is to keep the amount of settlement within tolerable limits (Mellor, 1969). Excess settlement may occur by any of the failure mechanisms described above, and in some situations any appreciable settlement may be regarded as 'failure'. In terms of ductile deformation, numerous authors, including Andersland and Ladanyi (1994) specifically define failure as the secondary creep inflection point of a complete classical creep curve. However, Mellor (1975) suggests that depending on the loading applied, the time to this point could effectively be infinite, and thus failure would theoretically never occur. Geduhn and Enss (2006) in a paper assessing the service life of a German Antarctic Station, defined failure in terms of station settlement and defined a stress amount that must 
be avoided in order to deny the onset of tertiary creep, which would eventually lead to creep failure. Figure 2.7 shows these regions on a classical creep curve.

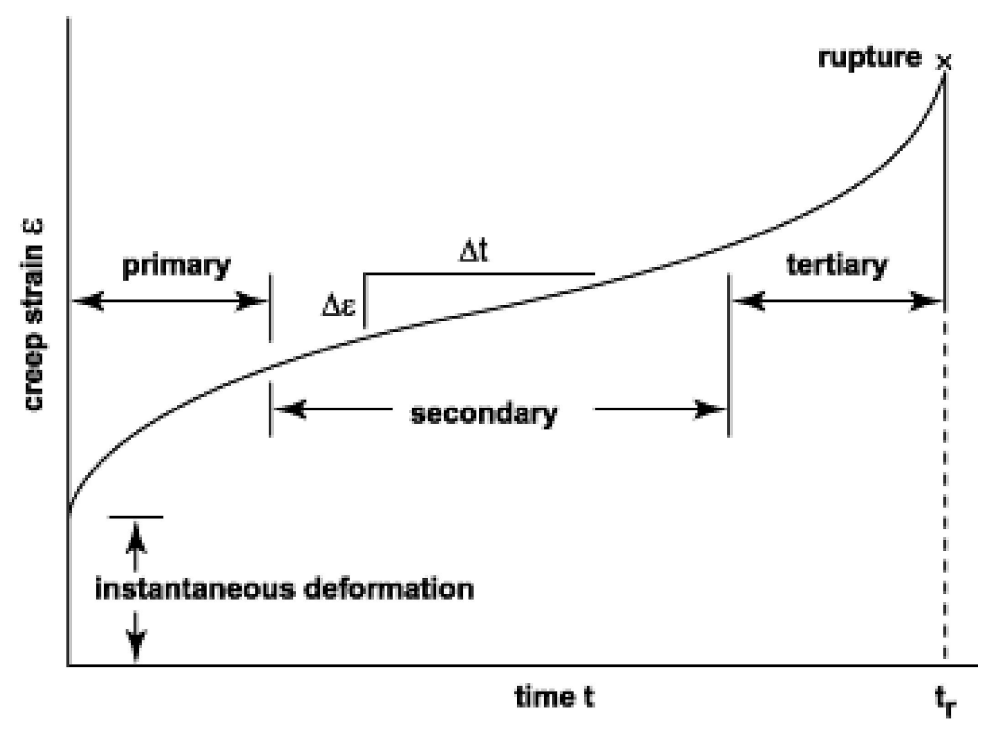

Figure 2.7: On a classical creep curve, a failure strain may be defined at the strain when the secondary creep inflection point is reached. This defines the onset of tertiary creep where strain rate increases until rupture (from Tarr (2011)).

The definition of failure with respect to the bearing capacity of snow is ultimately best defined with respect to the nature of the envisaged testing and use of the snow.

\subsubsection{Hardness and Resistance}

Snow strength is often expressed in other terms such as hardness or resistance, hence examination of the relationship between these and other indices for strength is necessary. The Metals Handbook (Davis, 1998) defines hardness as "Resistance of metal to plastic deformation, usually by indentation" and this definition is consistent with others (Brown (1998) etc.). Although this definition invokes consideration of plastic deformation (rather than the brittle deformation expected via CPT) it serves to confirm that hardness and resistance to penetration can be considered the same thing.

The relationships between typically measured snow strengths are examined later in Section 2.2.2. Recalling the envisaged behaviour of snow whilst undergoing penetration as described in Section 2.1.4, how do these strengths relate to hardness, the proxy for strength determined via penetration?

Mellor (1975) after analysing the physical nature of the penetrative process suggested that a linear relationship would be expected between strength and the specific energy (work / volume) of penetration. Further, with reference to a similar procedure derived from the field of rock mechanics, Mellor (1972) suggests that there are sound reasons for seeking correlation between ram resistance and uniaxial compressive strength in snow. 
Gibson and Ashby (1997) say that when a porous foam is indented by a flat punch, the cells beneath the punch collapse in the direction of loading but limited lateral distortion occurs, so the resistance measured in hardness is the same as the compressive strength of the material. McClung and Schaerer (1993) state that "Hardness is actually a measure of strength in compression", Kartashov (1965) states that "the limit value of stress under quick application of load can be considered as a criterion of snow strength" and that this is identical in the physical sense to "the limiting load of indentation, which in turn is nearly equal to the hardness value obtained by a cone penetration test" (not the same cone penetration test discussed herein). Lee and Huang (2010) also state that for large diameter penetrometers (where penetrometer size is $\gg$ grain size), measured resistance approaches the compressive strength of the snow.

So the hardness or resistance obtained via penetration is accepted to be equivalent to the compressive strength of the snow. Is this measure of compressive strength confined or otherwise? The definition of compressive strength defined above is for in situ material which is experiencing some level of confinement, consistent with the overburden pressure at which any test is conducted. Snow hardness can therefore be considered equivalent to the semi-confined compressive strength of snow, even though variations in confinement will probably affect this relationship, depending on the density of the snow under test.

Additional relationships between hardness obtained via penetrative instruments and hardness derived via other methods such as the hand-hardness test (de Quervain, 1950) are considered further in Chapter 6.

\subsubsection{Types of strength}

As noted earlier, snow will generally fail in shear even though the nature of the test may be compressive, tensile etc., but how is such testing typically conducted and how are these various forms of strength related?

1. Laboratory shear strength tests are generally conducted using a shear-frame in which the snow is sheared in one plane with no applied normal stress; similar tests are also conducted in the field using a manually loaded shear frame (see Perla et al. (1982), Jamieson and Johnston (2001) amongst others),

2. Unconfined compressive strength tests are conducted on cores extracted from the snow on strength testing equipment; again these tests may be conducted in the laboratory or in the field. (A description of an attempt at such testing conducted at Halley is described in Chapter 3.);

3. Triaxial testing allows confined compression strength testing (typically of cores) and is usually conducted in the laboratory, and 
4. Tensile testing can be conducted in the field by isolating a snow column and then using shear frames in opposition, or by other methods in the laboratory through the use of specific tensile testing equipment (McClung, 1979), or in a centrifuge (Upadhyay et al., 2007).

The relationships between these strengths are now briefly discussed.

Mellor (1975) commenting on his collated data for snow undergoing rapid loading says that "Broadly speaking, tensile and compressive strength (under uniaxial loading) are equal at low densities, whilst at the density of solid ice the ratio of compressive strength to tensile strength is about 5"; these data are also presented in more contemporary work by both Shapiro et al. (1997) and Marshall and Johnson (2009). Schulson and Duval (2009) note that the compressive strength of ice is greater than the tensile strength "by a factor of five to six" at $-10^{\circ} \mathrm{C}$ and Petrovic (2003) notes the increase in tensile strength with increasing snow density, but does not provide comparison with a compressive strength. It appears that a density-dependant relationship between the tensile and compressive strength of snow can be shown, but how are compressive and shear strengths related?

Via the Mohr-Coulomb failure criterion, if there is no normal stress (or if frictional strength is yet to be mobilised) then shear strength equals cohesion. Via this same criterion, under uniaxial compression, assuming no confining stress, then the maximum shear stress $(\tau)$ will act on a plane inclined $45^{\circ}$ to the applied axial load $\left(\sigma_{1}\right)$, and will equal half the major principal stress $\left(\tau=\sigma_{1} \sin 45^{\circ} \cos 45^{\circ}\right)$; this is consistent with Mellor (1975). (Compare this to Von Mises' theory (more regularly applied to ductile failure) where under uniaxial stress conditions, shear stress equals $\sim 0.57$ times the major principal stress, also assuming confining stress equals zero).

So in unconfined compression, failure will occur when shear stress exceeds cohesion, or thus when the principal axial stress exceeds twice the cohesion value (assuming no frictional contribution is mobilised). So, stress in compression at failure (compressive strength) should be equivalent to twice the cohesion value.

\subsubsection{Cohesion from CPT resistance}

Based on the discussion above, an assumption that forms the basis for my future attempts to derive the inherent cohesion or shear strength of snow from CPT resistance is now proposed.

It has been determined that resistance due to penetration is approximately equivalent to strength in compression and that strength in compression equals twice the cohesion. So, it might be deduced that the shear strength of the snow, equals half the measured cone tip resistance (neglecting geometry at this time).

This simple relation is based on the assumption that confining stress equals zero. In reality there will be some lateral stress and thus shear stress will be less than half 
the principal compressive stress, but as confining stress is small (typically one or two orders of magnitude less than applied uniaxial stress and typically only $\sim 25 \%$ of equivalent stresses in soil), this estimate appears appropriate. This assumption forms the basis for future attempts to derive an inherent cohesion or shear strength value from CPT resistance measurements, and is further explored in Chapter 7. This brief analysis has served to clarify the relationship between snow 'strengths' to better allow comparison with experimental CPT data.

\subsubsection{Effect of sample size}

Before moving on to discuss the instruments used in penetrative testing in snow, it is worth briefly commenting on the effect that sample size has on the strength assessment of snow via any means, as this topic is often raised in discussion on such matters. An understanding of size implications or otherwise is beneficial.

Weibull's theory (Weibull (1939b) \& Weibull (1939a)) based on the failure of the weakest link within a material suggests that as sample size increases the chance of a flaw (where fracture will initiate) being contained increases, and thus strength is expected to decrease with increased sample size. This theory is often the basis for assessing effect of sample size on the failure of brittle materials. Allegre et al. (1982) suggest "that fracture at the macroscopic scale is a consequence of accumulations of ruptures at lesser scales" and report work by Brace et al. (1966) etc. on the triaxial testing of rock, who found that two factors influenced the failure at the macroscopic level:

1. the nucleation of new cracks, and

2. the growth of pre-existing cracks.

Nucleation of new cracks will occur independent of the size of the sample, whereas the number of pre-existing cracks is expected to increase with sample size, and thus Weibull's theory would apply. Hoek and Brown (1997) in a review of sample size effect on rock strength concluded that an increase in sample size results in decreased strength "due to the greater opportunity for failure through and around grains, the 'building blocks' of the intact rock, as more and more of these grains are included in the test sample." However, debate continues on the effect of sample size in snow.

Sommerfeld (1974) after his work on avalanches concluded that "the mean strength of snow is a function of sample volume" and Perla et al. (1982) after assessing snow shear strength via various methods obtained significant deviation between plotted indices and thus noted that "sample size is important". Such findings support Weibull's original hypothesis.

However, Kirchner et al. (2004), after $\sim 600$ beam tests assessing the size effect in snow concluded that across a range of densities $\left(70-250 \mathrm{~kg} \mathrm{~m}^{-3}\right)$ the Weibull modulus 
remained constant, and that "unlike predicted by the weakest link hypothesis, there is neither a size nor a shape dependence of the strength of snow samples in the statistical sense." Chiaia and Frigo (2009) in their work on snow slab avalanches also reported the scale invariance in the behaviour of almost 5000 avalanches in France monitored in four years by Faillettaz et al. (Faillettaz et al., 2003) and noted the conclusion that "this particular behaviour may reflect the scale invariance of the distribution of snow shear strength" (Faillettaz et al., 2003).

One thing that is apparent from the two examples of non-Weibull behaviour noted above is that in both cases, (Kirchner et al. (2004) and Faillettaz et al. (2003)) the variability in sample size was not great. In Kirchner et al.'s case sample size only differed by approximately one order of magnitude and only three different sizes were tested, and in Faillettaz et al.'s work, it is assumed again that scales were not vastly dissimilar; all were of avalanche size. Hence it may be that the Weibull theory may hold at greater scale variance, or perhaps it applies more rigourously at microscopic scales where the influence of material structure, grain size etc. is more proportional to the size of the initiating defect.

This brief review presents inconclusive evidence for the impact of the size-effect on the strength of snow. Schulson and Duval (2009) examining fracture toughness in ice come to a similar conclusion, stating "whether size affects fracture toughness is not clear". Now, penetrative testing in snow, and then more specifically the cone penetration test are examined.

\subsection{Penetrative testing in snow}

Since the 1930's numerous penetrative instruments have been used to assess the strength of snow. A brief review of the major instruments that have been used is presented below, and then the history of the cone penetration test is specifically examined.

\subsubsection{Rammsonde}

The Swiss Rammsonde has for many years been used to test snow hardness. It was developed in 1939 by Haefeli et al., and consists of a rod with a conical tip, which is driven into the soil by means of a weight dropped onto the rod. The drop hammer is of a known weight, is dropped from a known height, and thus a ram resistance formula can be used to generate a ram hardness number, which describes the resistance in $\mathrm{kg}$ (Abele, 1963). Abele (1963) attempted to establish an empirical relationship between ram hardness and unconfined compressive strength (determined by an unconfined compression test apparatus), yet obtained a large amount of scatter. However, as discussed in section 2.2.1, Mellor (1975) expressed sound reasons for seeking correlation between the two parameters, and thus the use of a penetrative instrument (ostensibly measuring 
hardness) may still provide a valid proxy for snow strength.

\subsubsection{Thermo Resistograph}

Bradley (1968) describes the use of a snow resistograph, which is inserted to depth within the snow pack, rotated 90 degrees, and then manually withdrawn. The resistance as the device is withdrawn is measured by a spring in the handle of the resistograph, and thus a record of snow resistance as a function of depth can be generated. Bradley attempted to correlate the resistance data with a macroscale assessment of the snow pack compressive strength. However, although roughly equivalent data were generated, many unknowns in the conduct of the test meant that further development of both the instrument, and means of interpretation was necessary.

Building upon Bradley's work, the Digital Thermo-Resistograph (DTR) was developed by Dowd and Brown (Dowd and Brown, 1986). It uses a semi-conductor strain gauge load cell with a 60 degree cone in the end of a probe. A position sensor records the position of the probe, and the measured force divided by the projected area of the cone provides a measure of stress. It appears from Dowd and Brown's work that the probe is driven into the snow at a constant rate via a geared mechanism powered by an electric motor. Dowd and Brown note that the effect of insertion rate needs to be further studied, and their development of this instrument appears to have been the precursor for the development of more refined instruments such as the Snow Micro Penetrometer, described below. Pielmeier and Schneebeli (2003) state that "frequent malfunctions and lack of durability in the field are probably the reasons why only a prototype was built", and further go on to describe development work by Brown and Birkeland (1990) on another prototype, which was also discontinued owing to durability problems. Although the series of digital resistograph instruments proved promising in the assessment of alpine snow pack resistance, their use in assessing snow strength on a larger scale appears deficient because of depth and durability limitations.

\subsubsection{Snow Micro Penetrometer (SMPT)}

The Snow Micro Penetrometer developed by Schneebeli and Johnson in 1997 (Schneebeli and Johnson, 1998) is perhaps the most mechanically advanced snow penetrative instrument currently used in assessing in situ snow strength. It uses an electric motor drive to push the conical instrument into the snow at a (largely) constant rate, and is the most expensive of the force-resistance probes (Floyer, 2006). The penetrometer was designed mindful of the data obtained by Fukue through his thin blade penetration tests (Fukue, 1977) that showed that an instrument of smaller dimensions may be more responsive to changes in snow microstructure. The instrument consists of a cone with a diameter of $5 \mathrm{~mm}$, which is connected to a $3 \mathrm{~mm}$ diameter drive rod. The SMTP can be driven to depths of approximately $4 \mathrm{~m}$, and generates data of penetration re- 
sistance $(\mathrm{MPa})$ verses depth, with the ability to resolve snow layers down to $3 \mathrm{~mm}$ in thickness. Schneebeli and Johnson have invested considerable effort in interpreting the penetrometer-resistance profiles. Derivation of the compressive strength of snow has been described by Kronholm (2004) based on work by Johnson and Schneebeli (1999) who performed a statistical analysis on the penetration of the Snow Micro Penetrometer into snow. They derived an equation relating the angle of the Snow Micro Penetrometer tip and the frictional coefficient of the snow grains; the applicability of their work to CPT is considered in Chapter 7. Although the SMPT appears a highly suitable instrument for determining in situ snow strength, a number of considerations may impact its successful use.

Firstly, the consistency of its insertion rate is questionable. Kronholm (2004) notes that operator weight is required to maintain the penetration of the cone tip when hard layers are encountered, causing either an increase or decrease in the penetration rate; thus the penetration rate can not be assumed constant and any interpretation of data must be made cognisant of this.

Secondly, the dimensions of the probe mean that its robustness if inserted to depth in dense polar snow is questionable; a larger shaft and thus cone diameter is desirable. Verbal communication with O. P. Mattila, April 2008, regarding the use of the SMPT on the Vestfonna ice cap, Svalbard, suggested that the SMPT was ineffective in penetrating the hard polar snow. Rejection apparently occurred at depths of less than 10 $\mathrm{cm}$, and the drive mechanism was unable to maintain penetration into the snow.

The SMPT is a well developed and proven instrument which appears extremely useful in assessing the microstructural layering of alpine snow depths to a limited depth. However, its inability to maintain a constant drive rate and its relative fragility limit its use as a suitable in situ snow strength assessment device for polar snow. Although larger probes may be less responsive to snow microstructure, the ability to penetrate polar snow to depth at a constant rate using reliable commercially proven equipment is advantageous.

\subsubsection{Other penetrative instruments}

Abele (1990) notes the development in the 1960s by the US Navy Civil Engineering Laboratory of a blunt-tipped rod that was driven rapidly into the snow via the hydraulic system of a small tractor. Abele comments that such a test is in principle desirable, because of its speed and simplicity, but also states that the concept was not taken past the initial and apparently promising experimental stage.

The SABRE probe is a portable, variable-speed, digital, round-tipped penetrometer developed by Mackenzie and Payten (2002) that measures the force resistance of snow, as well as snow temperature. It is designed for detecting snow layers, particularly with the purpose of identifying weak layers for the assessment of slope stability. The probe is novel in that although it is manually inserted into the snow at variable rates, an internal 
accelerometer measures accelerations and thus velocity and displacement, and hence a plot of penetrative force verses depth can be generated. The SABRE probe is light, portable, and easy to use (Floyer and Jamieson, 2006), and although it may continue to prove of value in snow slope assessment, its manual and thus variable insertion rate, to a limited depth, means that it is not a suitable instrument for assessing the strength to depth of dense polar snow.

Floyer and Jamieson (2006) recently provided an update on digital penetrometer technology, discussing the SMTP, the SABRE, and the Capacitec Probe. The Capacitec Snow Probe measures the dielectric permittivity of snow, an analogue for density, but not strength.

R. Alger of the Keweenaw Research Centre at Michigan Technological University (email communication, 28 October, 2008) stated that he had developed an automated penetrometer in 2005, and obtained over 200 profiles across the Antarctic continent, at depths to one metre (although greater depth is possible with extensions). The device is electrically driven and mounted on the front of a tracked snow vehicle. Comparison with both density profiles and standard Rammsonde profiles is intended, however, limited data reduction has occurred, and limited funding apparently precludes further development at this time.

The nature of penetrative instruments historically used in testing snow has briefly been examined and now the cone penetrometer, the instrument that provides the foundation of testing within this work, is described.

\subsubsection{The Cone Penetration Test (CPT)}

The Cone Penetration Test (CPT) was developed in the Netherlands in the 1930s (Brouwer, 2007) and was initially known as the Dutch cone test. The equipment employed consisted of a steel rod with a cone attached to its tip, that was manually pushed into the ground. The test is used for profiling soil layers, and for deriving soil properties such as density, shear strength, angle of internal friction and cohesion (Schaap and Fohn, 1987). The mechanical cone penetrometer evolved over time to incorporate a sensor for measuring sleeve friction in the 1950s, before development of an electric cone by Fugro in the 1960s that was capable of measuring cone resistance and sleeve friction continuously during penetration (Schaap and Fohn, 1987).

The modern cone penetrometer can be fitted with sensors which measure temperature, conductivity, pore pressure, magnetic signature etc., however, the standard device measures cone resistance and sleeve friction. A CPT is performed by pushing a cone of known dimensions into the soil at a known rate (typically $20 \mathrm{~mm} \mathrm{~s}^{-1}$ ) via hydraulic pushing equipment. An acquisition system continuously records the data generated by the electrical strain gauge load cells that are positioned behind the cone and the friction sleeves (Lunne et al., 1997). Cone penetrometers measure cone resistance $q_{c}$ $\left(\mathrm{N} \mathrm{mm}^{-2}\right)$, which is the total force acting on the cone $Q_{c}(\mathrm{~N})$ divided by the projected 
area of the cone, $A_{c}\left(\mathrm{~mm}^{2}\right)$. Measured parameters and interpretation of test results in soil are influenced by numerous factors including existing in-situ stresses, soil compressibility, particle size, and cohesion (Lunne et al., 1997). The interpretation of CPT results in media other than soil also requires careful analysis of test output.

Schaap and Fohn (1987) describe the use of a modified cone penetrometer that is manually driven into the snow, and generates profiles of cone resistance $(\mathrm{kPa})$ verses depth. Although Pielmeier and Schneebeli (2003) in their review of this technique note the complexity of the resistance signal, and the difficulty in interpretation, Lunne et al. (1997) in their review of cone penetration testing suggest that the test may have future practical use in snow. Ladanyi et al. (1991b) investigated the strength characteristics of ice using an electric cone penetrometer hence the use of the cone penetrometer in dense snow (which may behave rheologically in a manner approaching ice) is worthy of further examination.

The above section has briefly examined existing penetrometers that have been used in snow to derive a proxy for snow strength, and the standard electric cone penetrometer that has now become the standard in soil investigations, and was the instrument utilised in this research. Table 2.2 summarises the characteristics of these instruments.

Table 2.2: Summary of snow penetrative instruments.

$\begin{array}{lllllll}\text { Penetrometer } & \begin{array}{l}\text { Diam. } \\ (\mathbf{m m})\end{array} & \begin{array}{l}\text { Area } \\ \left(\mathbf{c m}^{2}\right)\end{array} & \text { Drive } & \begin{array}{l}\text { Depth } \\ (\mathbf{m})\end{array} & \begin{array}{l}\text { Weight } \\ (\mathbf{k g})\end{array} & \begin{array}{l}\text { Resolution } \\ (\mathbf{m m})\end{array} \\ \text { Rammsonde } & 40 & 12.56 & \text { Weight } & 3 & 4 & 10 \\ \text { Modified CPT } & 11.3 & 10 & \text { Manual } & 4 & 20 & 10 \\ \text { SMPT } & 5 & 0.2 & \text { Electric } & 2 & 8.5 & >1 \\ \text { Resistograph } & 25.2 & 5 & \text { Electric } & 2 & 2.4 & <10 \\ \text { Blade (Fukue) } & 1.2 \times 0.6 & 0.007 & \text { Electric } & 0.1 & \text { Unk. } & <1 \\ \text { SABRE } & \text { Unk. } & \text { Unk. } & \text { Manual } & 1 & \text { Unk. } & \text { Unk. } \\ \text { NCEL Probe } & \text { Unk. } & \text { Unk. } & \text { Hydraulic } & 2 & \text { Unk. } & \text { Unk. } \\ \text { Capacitec } & \text { Unk. } & \text { Unk. } & \text { Electric } & 2 & \text { Unk. } & \text { Unk. } \\ \text { Electric CPT } & 35.6 & 10 & \text { Hydraulic } & >10 & 1000 & 10\end{array}$

Examination of this table suggests that the only choice for penetrating hard polar snow to a depth of a number of metres using a proven and reliable technology to obtain a strength proxy for snow is the electric cone penetrometer.

\subsection{Summary}

This chapter provided a brief yet directed review of the nature of snow and the typical means of assessing snow strength, particularly via penetrative instrument. It also provided a brief review of the historical and the current state of such assessment, and showed why the use of robust and proven commercially available CPT equipment is sensible for assessing the strength of polar snow. The assessment of a snow strength proxy via CPT is both necessary and viable, hence the remainder of this thesis addresses 
this research deficiency and provides a foundation from which the rapid and accurate assessment of the stratigraphy and strength of hard polar snow packs can be derived via Cone Penetration Testing. The methods I adopted in my research are described next. 


\section{Chapter 3}

\section{Methods}

The primary aim of this research was to perform cone penetration testing in polar snow in order to determine whether snow strength can be obtained via this technique. A secondary aim was to investigate how the CPT data may be used to estimate useful engineering information such as surface bearing capacity. Specific testing routines that were conducted and the rationale for the testing are listed below:

1. Pilot-study into penetrative testing in polar snow using the Fugro mini penetrometer - to provide additional data to assist in interpreting phenomenon observed during cone penetration testing,

2. Cone penetration testing - the primary method of addressing the research aims, and

3. Supplementary subsurface testing to assist in the interpretation and application of CPT, including:

(a) Density assessment - to enable comparison of CPT data with stratigraphy and enable computation of density-derived strength indices,

(b) Compressive strength testing - to enable direct comparison of CPT resistances with snow compressive strength,

(c) Shear strength testing - to enable direct comparison of CPT resistances with snow shear strength,

(d) Pressure bulb testing - to assist in estimating vertical stress transmission in layered snow, thus assisting in the estimation of surface bearing capacity in layered snow, and

(e) Ground penetrating radar (GPR) testing - to verify the correlation between GPR-derived stratigraphy and CPT resistance, thus verifying the ability to spatially extrapolate snow mechanical properties derived from point CPT measurements. 
Most of the investigations were conducted in Antarctica in January/February 2010. This chapter examines the methods outlined above and specifically addresses the way in which I conducted my CPT and supporting investigations. The Fugro mini penetrometer testing is initially examined, then the primary cone penetration testing in Antarctica; additional specific questions that I was hoping to address through the testing are also introduced. Finally, the supporting testing is introduced. This chapter only presents the methods employed in obtaining data; all results are presented in the next chapter.

\subsection{Fugro mini penetrometer testing}

Prior to conducting my primary period of field work in Antarctica, the opportunity existed to conduct a pilot study in Greenland investigating penetrative testing using an innovative hand-held probe. This testing subsequently proved inferior to the major field CPT that was conducted in Antarctica, but it provided additional data to allow better examination of some facets of snow behaviour during penetration.

Testing was conducted at four locations in the immediate vicinity of a field camp located on the western boundary of the southern Greenland ice sheet, within the ablation zone, at an elevation of 1169m (Figure 3.1). Access was gained by air via Kangerlussuaq. Snow depth at this location was limited $(30-60 \mathrm{~cm})$ overlying a seasonal layer of water ice, as the camp was located on a frozen inland lake. The approximate location of the four testing sites is shown in Figure 3.2.

The penetrometer is manufactured by Fugro Engineers B.V., Netherlands, and was designed as a lightweight, portable, penetrative unit, for use in snow and ice. The penetrometer is battery operated $(3 \times 1.5 \mathrm{~V}$ AA batteries $), 50 \mathrm{~cm}$ in shaft length, and has a $1 \mathrm{~cm}^{2}$ cone, with an included angle of $60^{\circ}$, see Figure 3.3 .

The cone is robust and simple to operate. The cone is switched on and then forced manually into the snow or ice surface by the operator. Data is recorded at a rate of $1 \mathrm{~Hz}$ and stored internally, and upon completion of testing the cone is turned off. The cone has no means of measuring depth or location, and will continue recording during both penetration and retraction as long as it remains turned on. The cone is strictly a subtraction cone (see Glossary) and records cone tip resistance $(\mathrm{kN})$, tip resistance plus sleeve friction $(\mathrm{kN}$ ) (via a $44 \mathrm{~mm}$ long, strain-gauged sleeve, located immediately to the rear of the cone tip, see Figure 3.4), and ambient temperature $\left({ }^{\mathrm{O}} \mathrm{C}\right)$.

The temperature sensor is enclosed within the cone handle and is susceptible to warming during use. It does not measure material temperature. Data from the cone can be downloaded onto a standard PC using proprietary Fugro software, and converted to an Excel spreadsheet file for analysis. Cone specifications are in Table 3.1.

Conduct of tests using the hand-driven penetrometer was very simple. After identifying the test area the probe was turned 'on' via the rotary switch on the top of the 


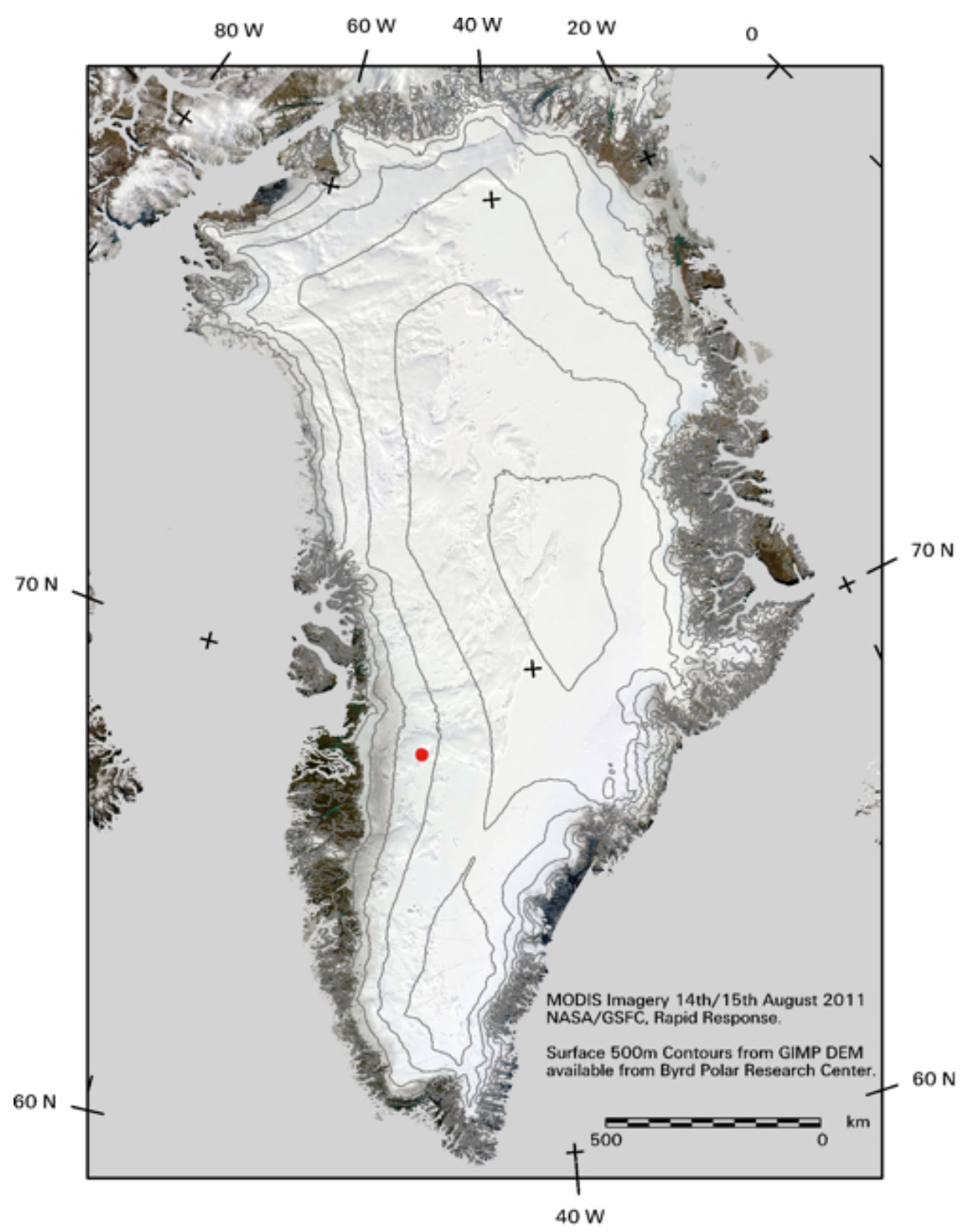

Figure 3.1: Approximate test location (red dot), southern Greenland.

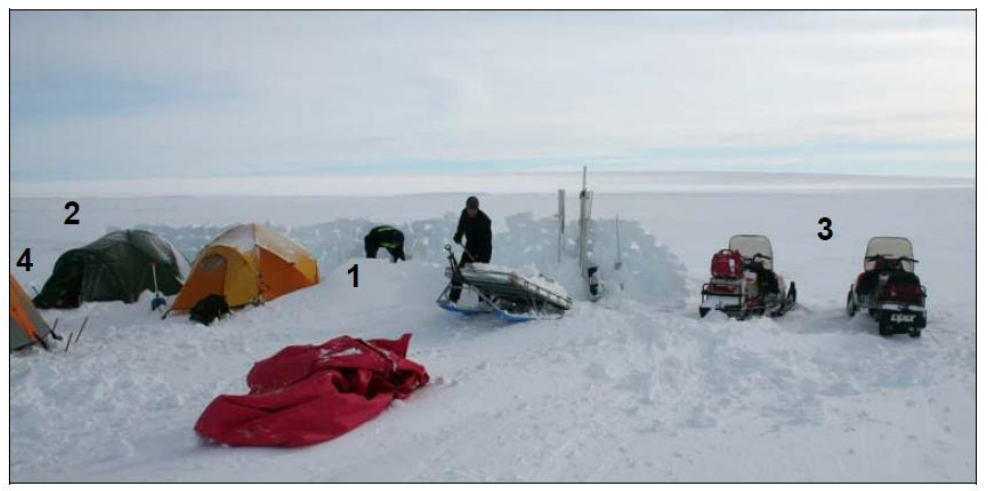

Figure 3.2: Greenland testing sites 1 - 4 (approximate location).

unit, and then the probe was manually pushed into the snow at as constant a rate as possible. Upon reaching the required depth the cone was then withdrawn and turned 'off'. Test data were downloaded as necessary using the serial/USB cable accessed by 


\section{$20 \mathrm{~cm}$}

Figure 3.3: Fugro mini snow penetrometer.

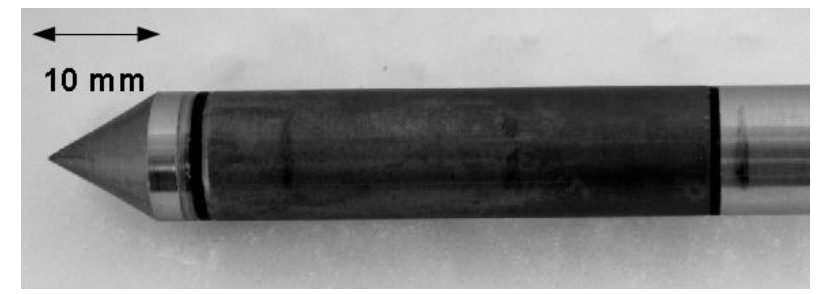

Figure 3.4: Fugro mini-penetrometer friction sleeve.

Table 3.1: Fugro mini snow penetrometer specifications.

Equipment number

Size

Weight

Device Type

Load

Voltage

Endurance $\left(1^{*} / \mathrm{sec}\right)$

Memory

Data tap protocol

Sample Rate

Channels

* Estimate. Battery life depends on temperature, humidity and battery condition.

removing the probe on/off switch.

The object of the field testing was to evaluate the operation of the snow penetrometer and to gain initial insight into the interpretation of cone penetration in polar snow. Many of these queries are also relevant to the larger-scale cone penetration testing conducted in Antarctica, and are considered more comprehensively in the next section. Specific questions to be addressed included:

1. Can the probe penetrate hard polar snow?

2. Can the probe be pushed into the snow at a constant rate?

3. What is the spatial variability of the data?

4. Does measured tip resistance vary with penetration rate?

5. How does sleeve friction vary with density?

6. How does tip resistance vary with density?

7. Was age hardening of the snow evident? 
8. What is the effective area of the cone?

9. Does a compacted zone appear ahead of the cone?

The Fugro manual snow penetrometer performed faultlessly throughout the testing programme. Operating temperatures were at times below $-20^{\circ} \mathrm{C}$, but data retention was unaffected. Although the cone is limited in dimension, sampling rate, and penetrative mechanism, it appears an ideal, lightweight, robust and portable instrument for initial penetrative investigation into shallow snow environs.

The primary problem encountered whilst using the manual snow penetrometer was in trying to maintain a constant speed of penetration. Extra force is required to penetrate 'hard' layers and in a stratified medium maintaining a constant penetration speed is very difficult.

A successful penetrative pilot-study was conducted in Greenland in April 2009 using a manually driven snow penetrometer provided by Fugro Netherlands. Although this campaign was only a pilot study, many questions necessary in understanding the use of a full-size penetrometer were considered, and significant insight was gained into the probable behaviour of a larger, hydraulically driven cone penetrometer. The methods employed in my primary field campaign in Antarctica are described next.

\subsection{Cone Penetration Testing}

In order to address the aims specified within the introductory chapter of this thesis, an extended period of time examining the use of CPT equipment in a polar field environment was desirable. To achieve this goal, logistical support was needed to provide access to, and time in such an environment, and additional support was necessary to acquire suitable CPT equipment for field testing.

Support from the British Antarctic Survey (BAS) provided the means of accessing BAS's Halley V Research Station in East Antarctica for the Austral Summer season $2009 / 10$, with equipment to be transported to and from the continent via ship, and additional discussion with Lankelma and Gardline Geosciences resulted in their provision of a custom-built CPT 'rig' built upon existing components, for use during an extensive period of CPT field-testing in Antarctica.

\subsubsection{Location \& Equipment}

CPT testing was conducted in numerous locations upon the Brunt Ice Shelf, predominantly in the immediate vicinity of the BAS Halley V Research Station. Approximate testing locations are annotated on Figure 3.5 and the location of each test is listed in Appendix A. The equipment that was used during CPT is described below.

CPT 'box'. In conjunction with Lankelma a steel 'box' was designed and constructed to provide a container in which a CPT operator could stand, and in which 


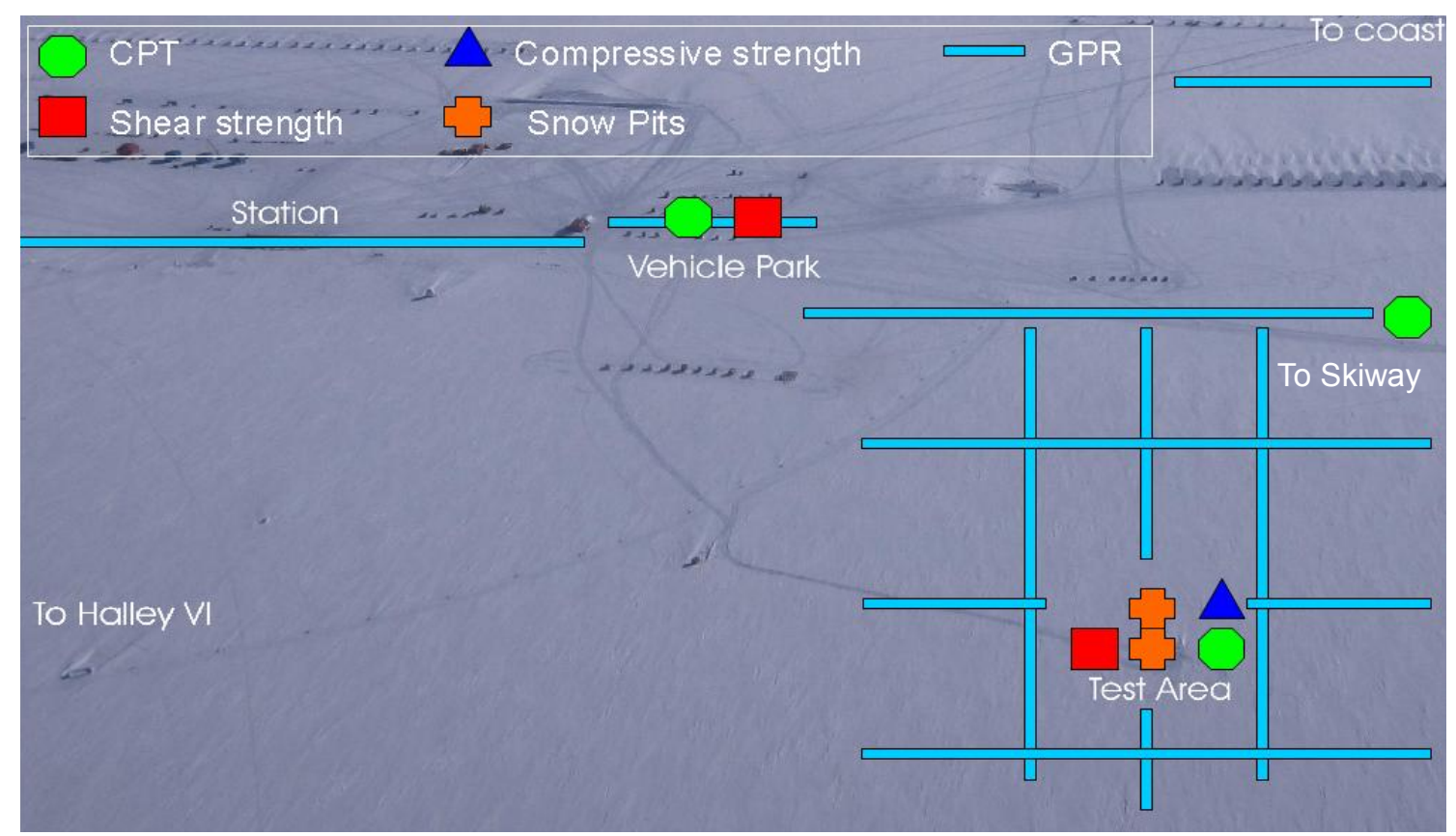

Figure 3.5: Main testing locations at Halley Research Station. Most testing was performed in the Test Area.

CPT and ancillary equipment could be stored and transported. The box was based around a tractor three-point hitch mounting plate made of $25 \mathrm{~mm}$ thick steel, that provided a rigid frame upon which CPT rams could be mounted. This plate (and thus the subsequent 'box' built upon it) was designed to attach to the category-three threepoint hitch of a standard agricultural tractor, such as those used by BAS at Halley Research Station. The box (designated UK11 by Lankelma) measuring $1.7 \mathrm{~m} \mathrm{x} 1.45$ $\mathrm{m} \times 1.35 \mathrm{~m}$ and weighing approximately $1300 \mathrm{~kg}$ when loaded is seen in Figure 3.6.

The box additionally consisted of a frame of box tubing which was welded to the main structural steel plate, onto which $1.5 \mathrm{~mm}$ gauge sheeting was welded. An additional $25 \mathrm{~mm}$ thick steel 'v-structure' was also welded within the back of the box, to provide additional support between the base on which the hydraulic rams are mounted and the vertical steel mounting-plate which is connected to the tractor.

On one side of the box was a rack to store twenty rods of $1 \mathrm{~m}$ length, and on the other side was constructed a small workbench on which the data gathering equipment and laptop computer could be placed, onto which was also mounted a vice to assist in rod attachment and removal. The box was secured by two steel-plate top-doors, and a rear door, all of which could be secured closed and padlocked, or latched open whilst the box was in use.

The hardness of the material through which a driven cone can penetrate depends on the reaction-force available from the pushing equipment. UK11 weighs only 1300 $\mathrm{kg}$ and because the standard three-point linkage only offers resistance to a downward force, an additional rigid-link was required to provide increased resistance between the base of UK11 and the top hitch of the tractor. A rigid link was modified from a John 


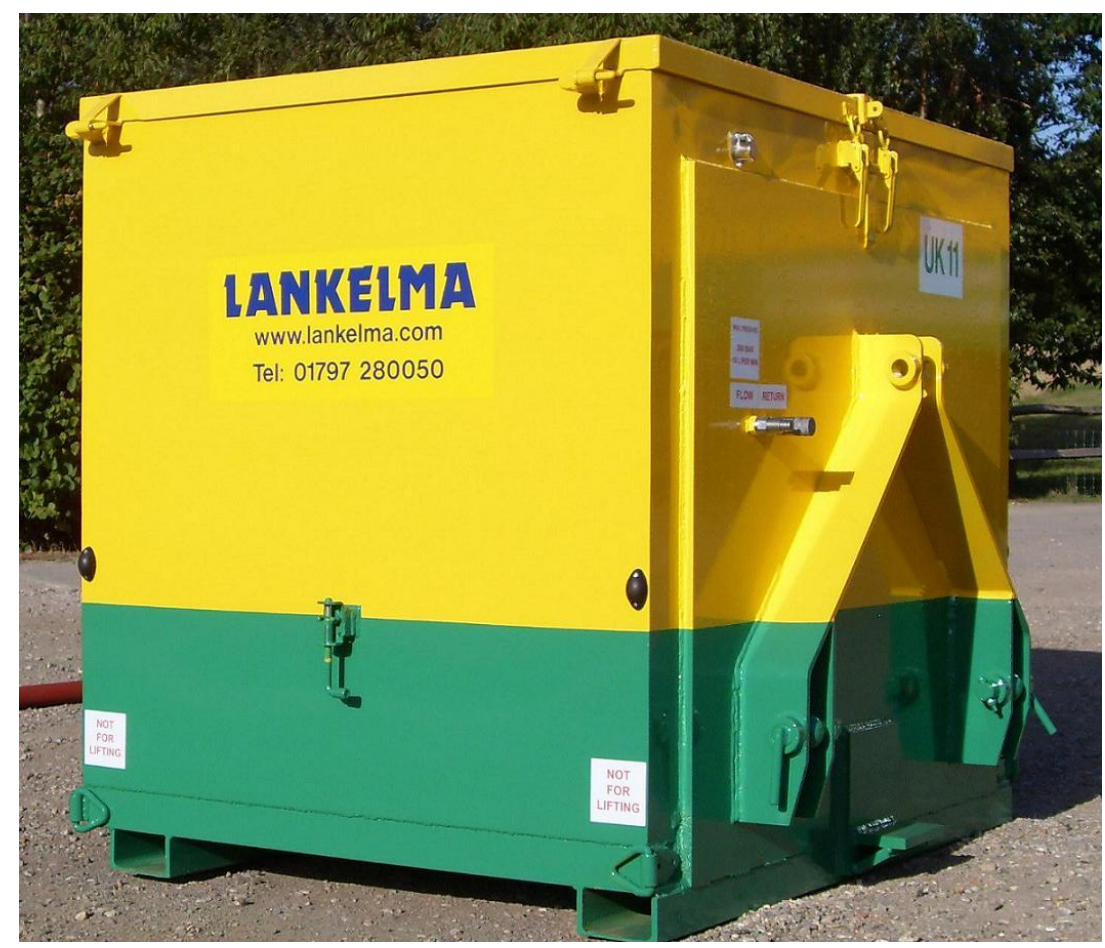

Figure 3.6: 'UK11' constructed by Lankelma within which CPT equipment was mounted. Note mounting points for tractor 3-point linkage and input ports for $12 \mathrm{~V}$ electrical power and hydraulics.

Deere category-three proprietary top link to enable the link to be attached to the spare top-link attachment point on a tractor, where it was connected via steel pin and lynchpin. The bottom of the rigid link was shaped, enabling it to slot into a steel ' $L$ '-shaped mounting bracket, welded to the base of UK11. During use the rigid-link could be left attached at the tractor, and then unscrewed and 'trailed' between subsequent testing sites. The John Deere tractor used in testing weighed approximately thirteen tonne of which approximately $55 \%$ lies over the back wheels, thus a potential reaction of up to eight tonne, or $80 \mathrm{MPa}$ was likely with this setup. The rigid-link can be seen in place in Figure 3.8.

Pushing equipment. A set of Lankelma's hydraulic 'basement' rams with a stroke of $500 \mathrm{~mm}$, bore diameter of $70 \mathrm{~mm}$, capable of a maximum pressure of 210 bar (to generate 10 tonne of force) were mounted within UK11 via a mounting-plate welded to the steel 'v-structure' within the box. These rams are manufactured by Julian Mills Engineering (UK) and can be seen in Figure 3.7 mounted within UK11. The rams were controlled via a two-way non-friction controlled hydraulic lever, mounted to the underside of the right-hand workbench within the box.

Hydraulic fluid was provided to the circuit within the box by directly attaching the box through two external couplings to one of the tractor's hydraulic circuits. The maximum permissible flow rate was $20 \mathrm{l} \mathrm{min}^{-1}$ and this was manually controlled by adjusting a hydraulic spool-valve mounted on the rear of the tractor, also accessible from inside the cab. No visual indication of flow-rate was used hence hydraulic flow-rate 


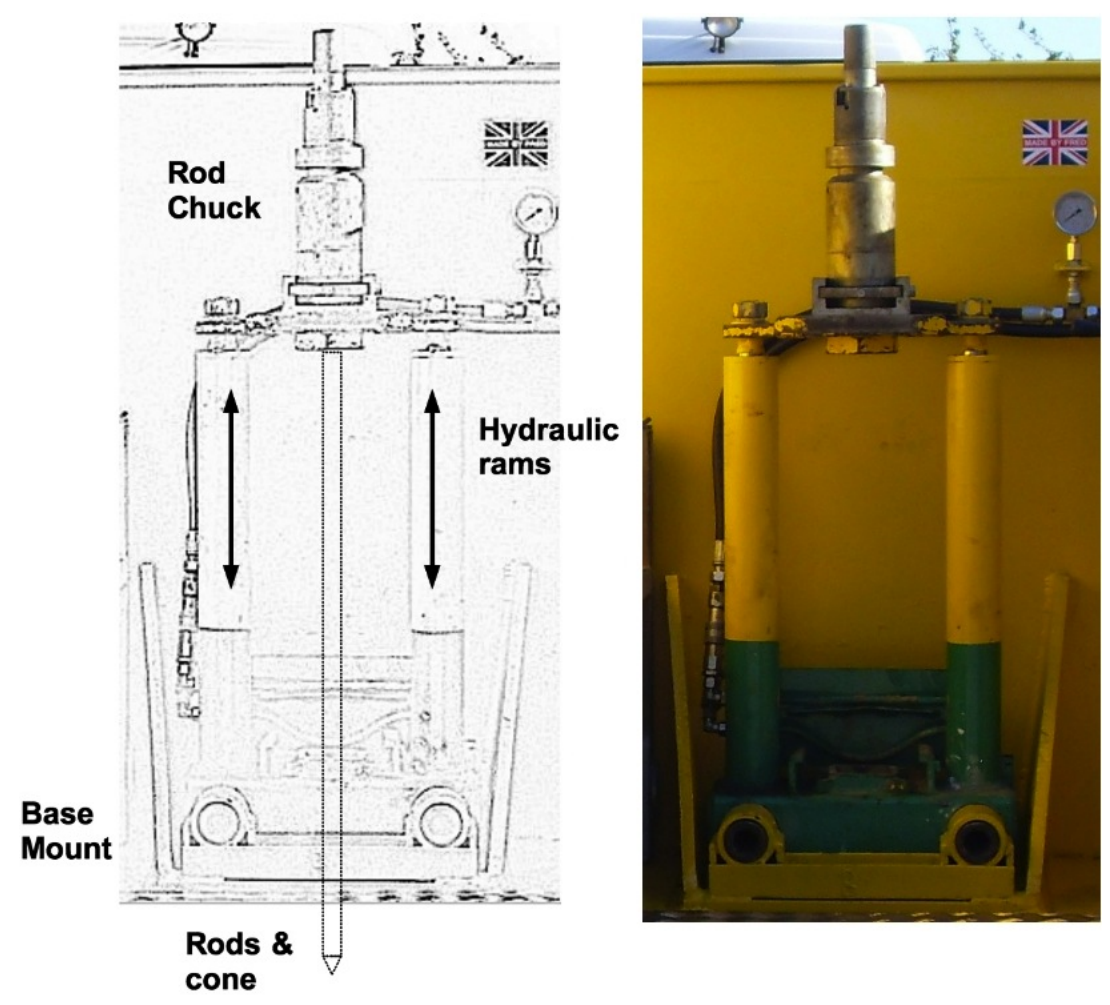

Figure 3.7: Photo of Lankelma 'basement' rams with rod clamp fitted, mounted within UK11; shown alongside a schematic, also depicting rods and cone position during use.

and thus ram-rate were established by a process of trial and error.

Driving force is transferred from the rams to the rods via means of a clamp which slots into a frame attached to the top of the rams. Numerous clamp types are available, although the lightweight clamp used is a simple ball-bearing type, borrowed from another Lankelma CPT rig, UK10, and manufactured by A. P. van den Berg. Three detents are manufactured within the clamp body, each selected by rotating the upper half of the clamp. One setting allows rods to be driven downwards but not upwards, one setting allows upward but not downward drive (for rod and cone extraction) and one allows the clamp to slide up or down the rods without gripping; the rods are gripped by a ring of ball bearings located within the clamp body. Maximum capacity of the clamp is 20 tonnes in either direction.

Rigid hollow rods are required to transfer the penetrative force from the hydraulicallydriven rams to the cone at depth. Standard $36 \mathrm{~mm}$ diameter steel alloy rods, each of length $1 \mathrm{~m}$, provided by A.P. van den Berg were used for this purpose.

Cones. Four specially produced scientific cones from Geopoint Systems BV were provided by Gardline Geosciences for the Antarctic CPT. Standard cone specifications are in Table 3.2 .

In order to standardise testing only one cone was used until it was damaged during opportunistic testing on the sea ice; a second cone was then used. Additionally, two manufactured flat alloy plates of diameters $36 \mathrm{~mm}$ and $120 \mathrm{~mm}$ were occasionally used for comparative testing. 
Table 3.2: GeoPoint 'Antarctic' cone specifications.

General Specifications

$\begin{array}{ll}\text { Cone Tip Section Area } & 1000 \mathrm{~mm}^{2} \\ \text { Friction Sleeve Surface } & 15,000 \mathrm{~mm}^{2} \\ \text { Total Length } & 280 \mathrm{~mm} \\ \text { Weight } & 2.7 \mathrm{~kg} \\ \text { Current Source } & 12 \mathrm{~mA} \\ \text { Working Temperature } & 0-60^{\circ} \mathrm{C} \\ \text { Storage Temperature } & -40-+85^{\circ} \mathrm{C} \\ \text { Connector } & \text { Lemo } 16 \& 18 \mathrm{p} \\ \text { Tip Resistance } & \\ \text { Range } & 20 \mathrm{kN} \\ \text { Accuracy } & 0.25 \% \mathrm{FS} \\ \text { Maximum Load } & 150 \% \text { of range } \\ \text { Cone Area Ratio } & 0.74 \\ \text { Sleeve Friction } & \\ \text { Range } & 3.75 \mathrm{kN} \\ \text { Accuracy } & 0.25 \% \mathrm{FS} \\ \text { Maximum Load } & 150 \% \text { of range } \\ \text { Sleeve Area Ratio } & 1.0(\mathrm{EA}) \\ \text { Pore Pressure } & \\ \text { Range } & 1 \mathrm{MPa} \\ \text { Accuracy } & 0.5 \% \mathrm{FS} \\ \text { Maximum Load } & 150 \% \text { of range } \\ \text { Filter position } & \mathrm{u} 1 \& \mathrm{u} 2 \\ \text { Inclination } & \\ \text { Range } & 25^{\circ}(\mathrm{biaxial}) \\ \text { Accuracy } & <2^{\circ}\end{array}$

Data recording. A Sick Stegmann wire-draw mechanism and incremental rotary depth-encoder was attached to the hydraulic rams via a mount on the lower right leg. This device (accurate to $0.02 \%$ of full stroke) enabled depth of the cone to be established; depth accuracy to $5 \mathrm{~mm}$ being limited by the data-logging equipment. Data recording was performed using a modified A.P. van den Berg 'Golog' data recorder. This robust data logger is designed for electrical cones with analog data transmission and has four input channels: cone tip resistance, sleeve friction, pore pressure and biaxial inclination (A. P. van den Berg, 2008). The data recorder normally records every $20 \mathrm{~mm}$ of penetration but was modified to allow recording at every $5 \mathrm{~mm}$ of penetration in snow. The Golog box was powered via cable connected to the tractor's 12 volt system, and connected to a laptop via a RS232/USB connection. Additional input cabling was from the cone and the depth encoder.

A standard Panasonic C32 Toughbook laptop computer running A.P. van den Berg's GOnsite! (A. P. van den Berg, 2008) windows-based software was used to manage CPT data acquisition and initial data manipulation. GOnsite! records the data on a depth or time basis and provides a real-time presentation of the CPT data on screen. It 
generates ASCII and GEF files, which can then be downloaded, stored, processed, converted and printed.

Both the cone tip and friction sleeve measure the resistive force in Newtons, and this is then converted into a stress measurement (MPa) within the cone, based on the assumed constant dimensions of the cone tip and the friction sleeve.

The cabling necessary for CPT operations included: a $30 \mathrm{~m} \mathrm{10-pin} \mathrm{geoscience} \mathrm{data}$ cable connecting the cone to the 'fly-lead' data cable (plus spares), a $\sim 2 \mathrm{~m} \mathrm{10}$-pin 'fly-lead' data cable connecting the $30 \mathrm{~m}$ data cable to the 'Golog' data recorder (plus spare), a data cable incorporating on/off switch between depth encoder and 'Golog' data recorder (data was only recorded when this switch was 'on'), a serial cable connecting the 'Golog' data recorder to laptop, and a power cable providing $12 \mathrm{~V}$ power to the 'Golog' data recorder and laptop from the tractor. Although numerous spares were carried for most cables, access to the Halley garage enabled ongoing cable maintenance/modification to occur as necessary.

Support from BAS, Lankelma, and Gardline geosciences enabled the assembling of a CPT system that could be mounted onto the three-point hitch of a standard tractor and be used to perform CPT in polar regions and other inaccessible regions of the world. The box, configured and fitted for use is shown in Figure 3.8. The box and associated CPT equipment proved secure, robust and versatile and was very well suited to the task.

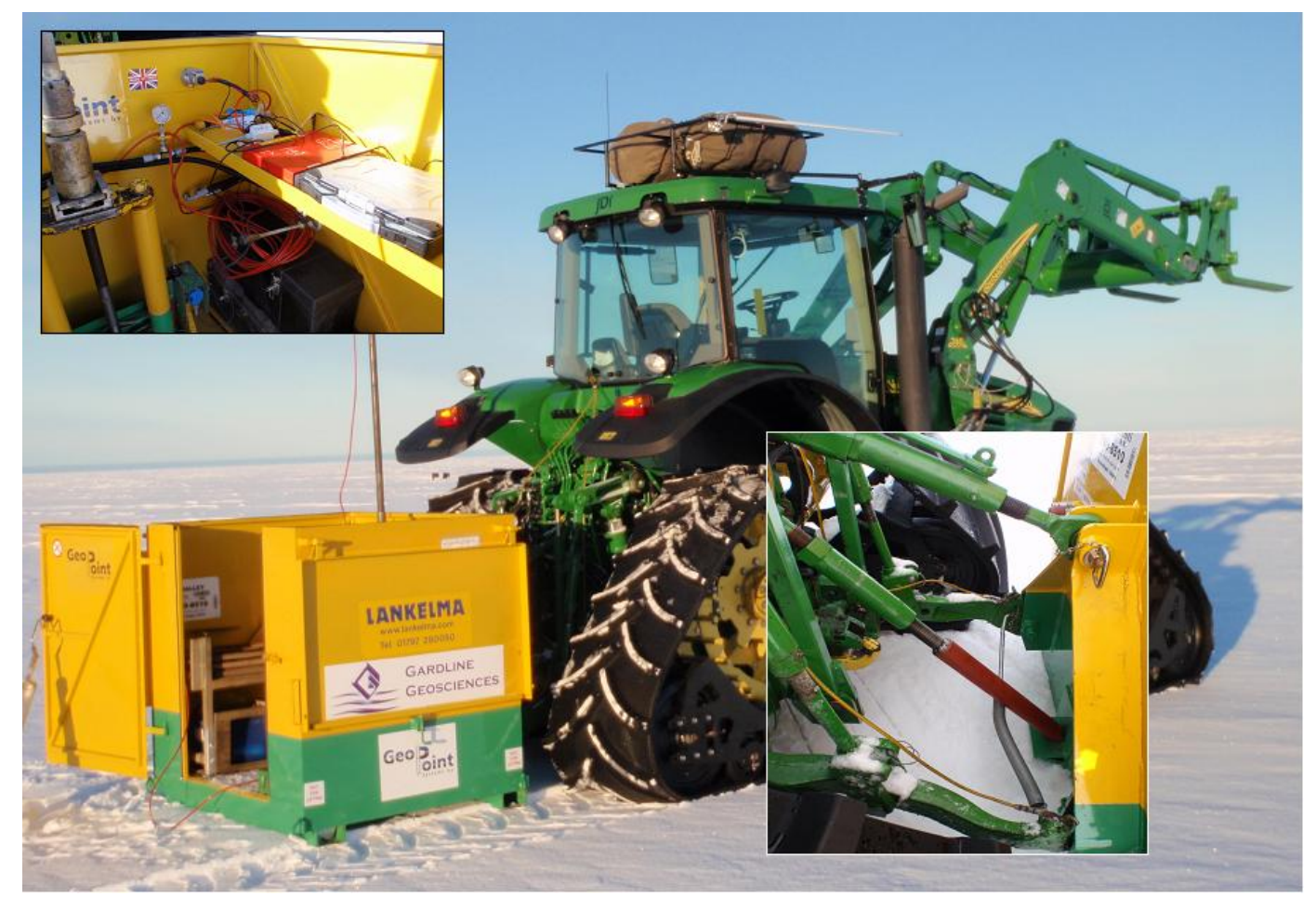

Figure 3.8: UK11 configured and fitted for CPT. Insets show rigid link installed and data collection equipment. 


\subsubsection{Calibration}

(Lunne et al., 1997) comprehensively outline the checks and recalibrations that should be carried out before the conduct of cone penetration testing. Steps to be considered include assessing the thrust machine and push rods for verticality and straightness, checking zero-load measurements, checking the cone for integrity and quality of seals, calibrating load cells and transducers and conducting temperature calibration. The dimensions and roughness of the cone and friction sleeve should also be checked. The manner in which each of these criteria was addressed is briefly discussed below.

The verticality of the CPT box and thus the thrust apparatus was visually verified before the commencement of each test. Such leveling often involved repeated lowering of UK11 to the snow surface in an attempt to achieve a vertical drive axis. However, more accurately a multi-directional spirit-level or similar should have been used in this assessment. Excessive misalignment was noted if evident.

Push rods provided by Lankelma were inspected for straightness and typically a selected five (out of twenty rods provided) were utilised for the testing. Zero readings were taken at the start and end of each test, and the precision of the measurements obtained was verified through initial spatially-confined tests. The supplied cones were new hence cone dimensions were exact and not deformed in any way, and limited additional wear was expected during testing in snow and ice; cone end area equally remained invariable throughout the testing. Similarly, seals and pore-water mesh were new, and were replaced as necessary between tests, if any excess wear or deterioration was noted. Cones were provided with calibration data and no additional recalibration was performed in the field. If a cone showed excessive zero-shift then an alternative (new) cone would be used, however, as previously noted, only two cones were used throughout the testing programme.

The cones were calibrated in the cold rooms of the Scott Polar Research Institute (SPRI) prior to deployment. Such calibration is particularly important because of the temperature sensitivity of the strain gauges used within a cone penetrometer (Rogers, 2006) and thus calibration was conducted to verify the zero-shift and linearity of the cones over the expected operational temperature range. Four cones similar to those that were used at Halley were tested for linearity at room temperature, and dissipation tests (i.e. monitoring readings with time as the cone adjusts to constant room temperature) were conducted on two cones, measuring the variation in tip resistance $\left(\mathrm{qc}_{\mathrm{c}}\right)$, sleeve friction $\left(\mathrm{f}_{\mathrm{S}}\right)$ and pore pressure $(\mathrm{u})$ over the temperature range $+23^{\circ} \mathrm{C}$ to $-20^{\circ} \mathrm{C}$. The variation in tip resistance at zero applied load is presented for two cones in Figure 3.9.

These results suggest that at temperatures greater than $\sim 8^{\circ} \mathrm{C}$, temperature compensation is achieved automatically by the cone temperature compensation system, but below this temperature, the zero-shift is proportional to the decrease in temperature. Consistent with the physics of thermal conductivity, it appears that the rate of temperature equilibration decreases as the temperature difference increases. The 


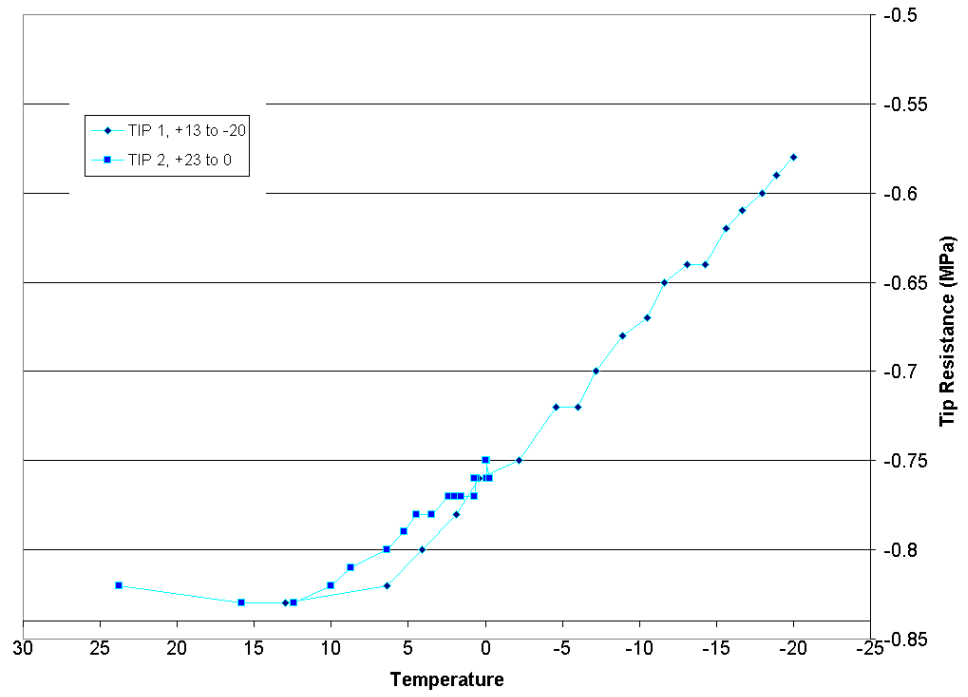

Figure 3.9: Variation of cone tip resistance zero-shift with temperature shows inadequacy of auto-compensation below $\sim 8^{\circ} \mathrm{C}$.

operational consequence of this preliminary testing is that below $8^{\circ} \mathrm{C}$, cones should be allowed to equilibrate with environmental conditions for at least one hour before use, and that at sub-zero temperatures, limited zero-shift is expected in a test duration of approximately ten minutes.

Additionally, plots of cone millivolt output versus applied load were generated for two cones at temperatures of $+20^{\circ} \mathrm{C}, 0^{\circ} \mathrm{C}$, and $-20^{\circ} \mathrm{C}$. In each of these tests, although limited data were available, the gradient in each test was essentially constant $(\mathrm{R}=$ $0.88)$, with the axial intercept varying with temperature as defined by the dissipation test data (Figure 3.9). This temperature calibration testing concluded that cone output $(\mathrm{mV})$ appears to vary linearly with applied load $(\mathrm{N})$ throughout the operational temperature range, with only the axial intercept varying with temperature.

The practical outcome of this testing was that all CPT equipment aside from the Golog box was stored in UK11 outside at ambient temperatures to minimise any variation because of temperature.

\subsubsection{Specific questions to be addressed in performing CPT}

Prior to conducting CPT, my research had highlighted numerous issues that needed to be understood or at least considered before conducting and interpreting my CPT; these issues are summarised below. Some of these considerations were explored within Chapter 2, however, these issues are briefly presented here amidst the practicalities of conducting CPT. They are considered in more detail in later chapters.

Rate effects. Dry snow is composed of ice and air, with the ice matrix defining material mechanical behaviour. Ice is a rate-dependant material (Schulson and Duval, 2009) hence it is probable that snow too is a rate-dependant material and historical testing supports this (Fukue (1977) and others). It is therefore appropriate to consider 
the effect of penetration rate on cone resistance and friction. Also, although penetration may be occurring at a particular rate the actual rate at which material ahead of the cone is being strained may be many times more than this rate. An understanding of this relationship is also necessary to accurately assess variations in measured stress with penetration rates.

Cone size \& shape. Although the effect of sample size in snow has been shown to be unclear, modeling by Johnson and Schneebeli (1999) and testing by others, including Whiteley and Dexter (1981) has shown that the effect of penetrometer size does affect measured stress. The shape of the cone (sharp or blunt) also affects the movement of material ahead of the cone and particularly in smaller diameter cones, grains fractured during penetration may be carried with the cone, potentially increasing the bearing surface of the cone (by perhaps $10 \%$ ), thus knowledge of both cone size and shape and effective area, is beneficial in accurately interpreting measured stresses.

Compacted zone \& cone sensing. As a cone is projected into snow, both observation and laboratory testing (Floyer and Jamieson (2006) amongst others) have shown that a plug or compacted zone of broken material may form ahead of the cone. Such a process has also been identified in the indentation of brittle porous rock (Leite and Ferland, 2001). This process requires investigation because such a compacted zone may act as an inert load-transferer and impact the depth-accuracy of stress measurements. In some soils the cone may 'sense' an approaching layer some 10 to 20 cone diameters before it reaches the layer (Lunne et al., 1997), supposedly via elastic deformation of the material ahead of the cone, thus resulting in force-transferral, but not fracture of these layers or particles. An understanding of this process is also clearly necessary in assessing the depth / stress relationship.

Confining pressure. Triaxial testing on low density snow shows no effect of confining pressure (Scapozza and Bartelt, 2003a). However, many other geomaterials including ice do display a dependency on confinement (Renshaw and Schulson, 2001). What effect does overburden (or confining pressure) have on cone resistance in mid to high density snow? Also, does sufficient frictional heating occur during penetration to cause pressure melting, and what effect might this have on testing methods and results, and how does this friction (never before measured in snow) vary with density, tip resistance etc. and what might be extracted from this data?

Cone penetration testing in polar snow, measuring both tip resistance and sleeve friction is not known to have previously been performed, hence there are many questions that require answering. The short list above outlined some of these questions, and the role of this thesis is to deal more comprehensively with most of these concerns. However, it is unlikely that all facets of cone penetration in polar snow can be suitably addressed within the confines of this document. The process of testing is described next. 


\subsubsection{Testing Process \& Problems Encountered}

The purpose of the CPT rig described in section 3.2 is to provide downward penetrative force to a series of steel rods via which a mounted cone can be driven into the ground at a known rate. The mechanical process of conducting each CPT is fairly standard and a set of Standard Operating Procedures (SOPs) outlining this process was produced, and are attached as Appendix B.

The field-testing described herein was the first use of this equipment. However, relatively few problems were encountered. Some issues that were experienced include:

1. depth encoder wire was prone to icing. This was alleviated by storing the encoder in a warm environment when not in use;

2. large amounts of vibration and lifting of the box and drive-mechanism because of slip-stick of the rods. This could have been largely eliminated by using a friction reducer on the rod string behind the cone;

3. vertical alignment of the rig was unable to be confirmed. Use of a spirit level on the rams may have proved beneficial; and

4. data-logging at every $5 \mathrm{~mm}$ of penetration meant that some extremes of tip resistance were not recorded.

The CPT equipment performed largely without fault in challenging operating conditions and minimal alterations are recommended for future similar use. Supplementary testing that was conducted to assist in the interpretation and application of the CPT data is now discussed.

\subsection{Density Assessment}

Whilst density may not be a complete descriptor for the mechanical behaviour of snow, snow strength indices are routinely compared with snow density (see Mellor (1975), Russell-Head and Budd (1989), Abele (1990) etc.). Therefore, density measurements are required so that snow 'strength' measured via CPT can be similarly compared. The two snow pits described below were primarily constructed to enable comparison with CPT data and to enable comparison of density-derived snow strength proxies with CPT data.

Two snow pits were dug adjacent to one another in the immediate vicinity of the Halley Station in an area that was not known to have previously been disturbed. The approximate location of this and other primary test sites can be seen in Figure 3.5. The $2^{\text {nd }}$ pit was located approximately $1.5 \mathrm{~m}$ to the East of the $1^{\text {st }}$ pit and was dug approximately three weeks later. The $1^{\text {st }}$ pit was $\sim 7 \mathrm{~m} \mathrm{long}, 1.6 \mathrm{~m}$ wide and $4.5 \mathrm{~m}$ deep and the $2^{\text {nd }}$ pit was $\sim 7 \mathrm{~m}$ long, $1.2 \mathrm{~m}$ wide and $2.5 \mathrm{~m}$ deep. Both pits were dug 
with the assistance of a mechanical excavator; the depth of the second pit was limited owing to the nature of the different excavator available at the time.

Snow samples were retrieved using two different cylindrical density tubes, one tube of length $247 \mathrm{~mm}$ and diameter $73 \mathrm{~mm}$ and the second measuring $250 \mathrm{~mm}$ with diameter $35 \mathrm{~mm}$. The large sampling tube was used when possible, but, limited layer thickness often necessitated the use of the smaller tube. Samples were weighed using an Acculab 'Econ' portable balance, precise to $\pm 1.0 \mathrm{~g}$.

Once the pit was dug, access was gained via tethered ladder and pencils were used to mark visible layers. All sides of the pit were examined in an attempt to verify the continuity of layers, and additional pencils were inserted if necessary. The grain size of each layer was then estimated by examining representative grains using a 10x lume and a $1 \mathrm{~mm}$ square snow card and then the hardness of each layer was estimated using de Quervain's standard hand-hardness test (de Quervain, 1950) where each layer is assigned a subjective measure of hardness: fist, four fingers, one finger, pencil or knife.

At least three samples of each layer were then retrieved using the appropriate snow density tube, and an assessment of weight and thus density was made. Visual characteristics of each layer were also noted throughout this process. A measurement of snow 'wetness' (as described by Bolognesi (2007)) was not performed because all samples were assessed as 'dry', and a subjective measurement of snow cohesion was obtained by examining samples using the $10 \mathrm{x}$ lume and a $1 \mathrm{~mm}$ square snow card.

The hardness of the snow within the pit, especially at depth, meant that occasionally the density tube had to be driven into the wall of the snow-pit using either a hammer or a boot. Such methods were not ideal as it was difficult to ensure that the tube was inserted 'squarely' without undue sample fracture or irregular compaction. Problematic layers are shown in Figure 3.10 (knife for scale).

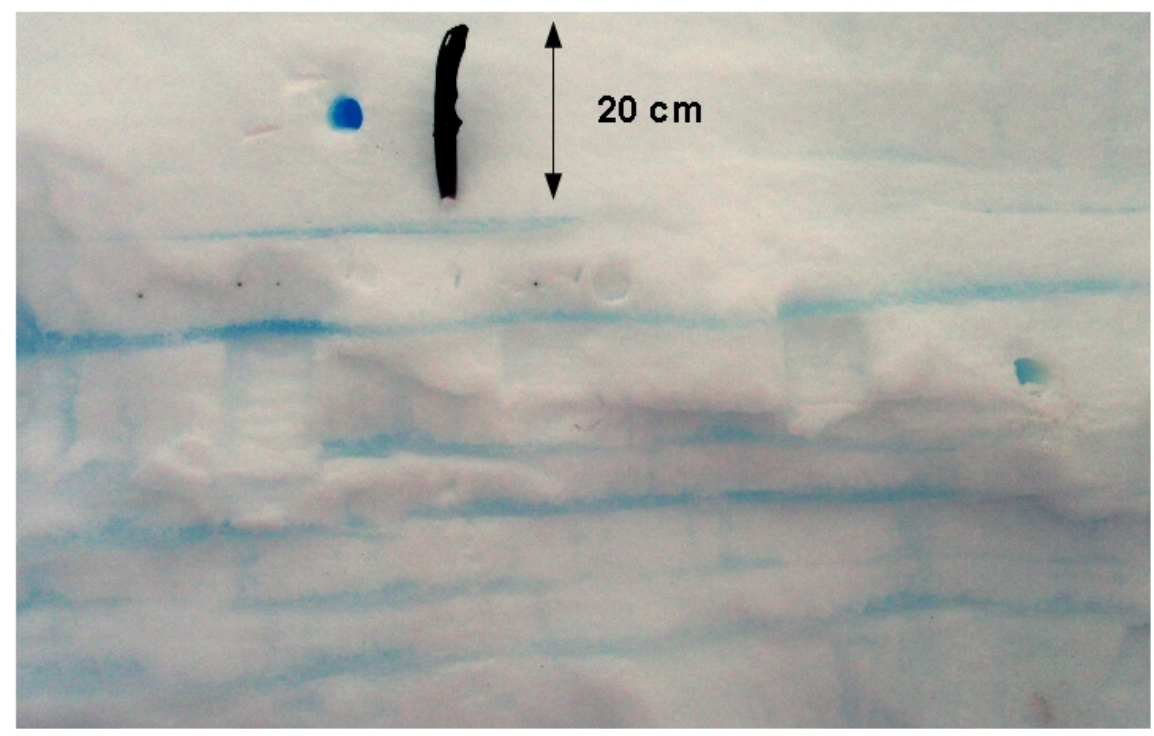

Figure 3.10: Layers of ice and hard snow hampered sample extraction at depths beyond $\sim 3 \mathrm{~m}$ (image contrast manipulated for clarity). 
Once the tube had been inserted into the snow, difficulty also arose in retrieving the sample tube; a pair of pliers was used to grasp the tube wall whilst the sample was removed in a twisting/pulling action. However, because of the smoothness of the tube, samples were often very difficult to remove and only $\sim 50 \%$ of samples were removed intact and tested. A better method of efficiently determining the density of hard snow to depth (5 to $10 \mathrm{~m})$ is necessary.

Gravimetric density measurements were taken from two adjacent snow pits to enable comparison of CPT resistance values with snow density. Although some problems were encountered because of the hardness of much of the snow, particularly at depth, representative density measurements to almost $4 \mathrm{~m}$ depth were achieved.

\subsection{Confined compressive strength testing}

Confined compressive strength testing of snow samples was conducted in an attempt to obtain a measure of strength for each identified snow layer, for comparison with subsequent CPT data. Samples were retrieved from the $1^{\text {st }}$ snow pit. Although it was originally envisaged that samples would be extracted from all layers, thin layers at depths greater than $3.3 \mathrm{~m}$ could not be sampled using available equipment and were thus excluded. Representative samples were retrieved from each layer of sufficient size using the large density tube (length $247 \mathrm{~mm}$ and diameter $73 \mathrm{~mm}$ ). It was originally envisaged that three samples would be tested for each layer, however, this was not always possible.

Ingenious use of the CPT equipment enabled it to be used in the strength-testing role. The standard CPT cone was replaced with a machined flat plate of diameter 36 $\mathrm{mm}$, the samples (encased within the tube) were then placed on a raised lower-platen of the CPT testing apparatus, and then the flat plate was manually lowered until it was level with the snow surface (see Figure 3.11). The sample was adjusted to ensure that the plate was central and the sample was vertical.

The data-logging software, A. P. van den Berg's "GOnsite!" (A. P. van den Berg, 2002) was started in 'dissipation test' mode, and then the plate was driven into the snow at a rate of $38 \mathrm{~mm} \mathrm{~s}^{-1}$. Each test was terminated once the plate was approximately $100 \mathrm{~mm}$ from the bottom of the sample. Data were recorded during both insertion and retraction of the plate and logging ceased once the plate was clear of the sample surface.

Two primary problems were encountered in conducting this testing. Firstly, the nature of the snow made it very difficult to remove 'whole' cylindrical snow sections for compressive testing. The need to drive the sampling tube in by impact often fractured the desired sample and sample integrity was often comprised. Seemingly complete cores may have been fractured, as in most cases impact was necessary to insert the corer. 


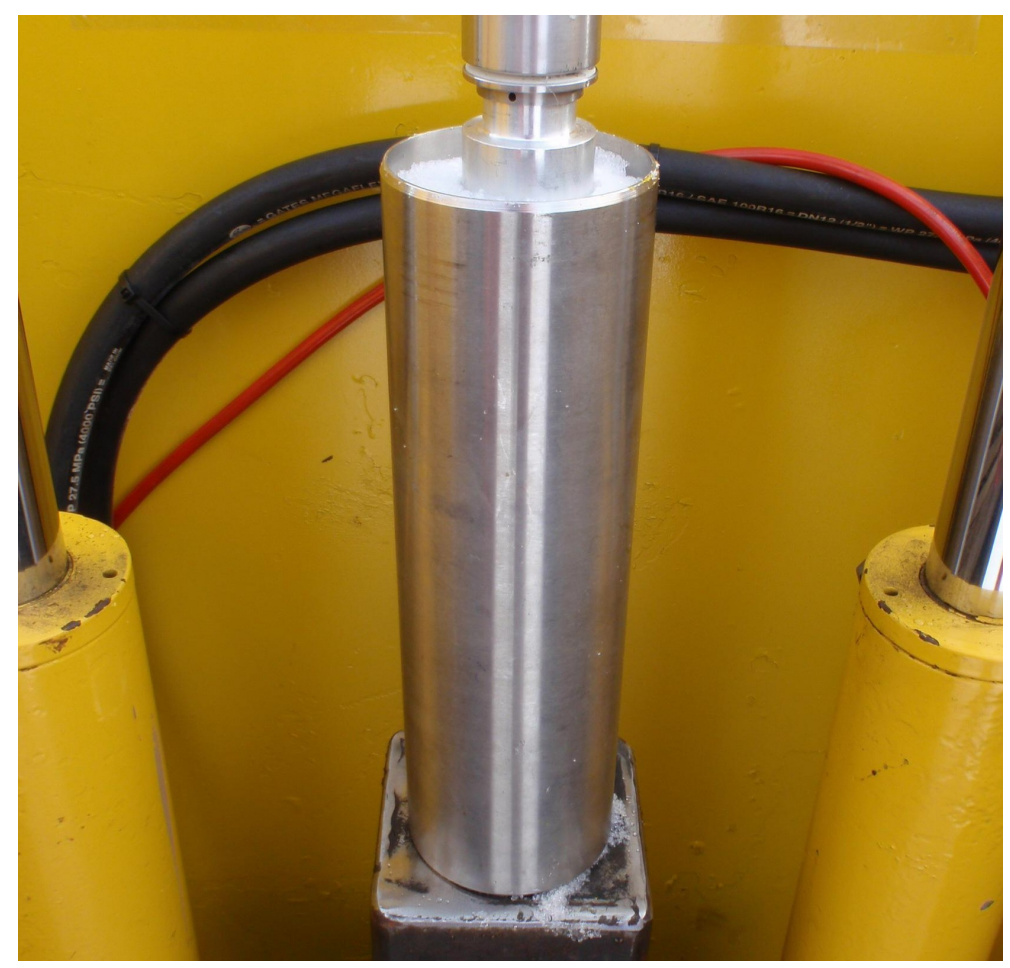

Figure 3.11: Confined compressive strength testing via plate insertion. A flat plate of diameter $36 \mathrm{~mm}$ is about to be forced into a snow sample contained within a steel cylinder of diameter $73 \mathrm{~mm}$ at a rate of $38 \mathrm{~mm} \mathrm{~s}^{-1}$.

Secondly, the testing equipment only enabled resistance data to be obtained every $1 \mathrm{~Hz}$, probably too coarse a data-collection rate to ensure that all failure 'peaks' were accurately recorded. This coarseness was accepted in the belief that although the nature of the equipment compromised accurate strength measurement, at least some useful data would be obtained for comparison with subsequent CPT data. An increased data collection rate whilst in 'dissipation test' mode would result in the CPT equipment becoming more versatile for such testing.

Confined compression testing of snow samples removed from the $1^{\text {st }}$ snow pit was conducted in an attempt to establish a relationship with snow density that could assist in the interpretation of resistance values obtained via CPT in the same snow pack, but the testing was hampered by the inability to consistently remove 'whole' samples for testing and the 'coarseness' of the available testing equipment. To obtain an additional strength proxy, further strength testing was conducted using a shear frame, as described next.

\subsection{Strength Testing (Shear Frame)}

Problems encountered whilst attempting to estimate snow compressive strength led me to consider alternative methods of estimating snow strength, preferably without using the CPT equipment, as the data sampling rate restricted the value of obtained data (whilst in 'dissipation test' mode). The assessment of snow shear strength through 
the use of a shear 'box' or 'frame' as described by Perla et al. (1982) was adopted, and I employed methods cognisant of the considerations espoused within, such as: acknowledgement that snow density is not always a fundamental predictor of bond development, that measured strength is dependant upon rate of loading, and that because in situ tests do not simulate pure or simple shear loading, the reported strength is to be interpreted as relative rather than absolute. However, typically shear frame testing is used to assess the strength of an identified weak layer, whereas in the testing I conducted, no weak layer was identified beforehand, and shear failure was assessed within any chosen snow layer, not necessarily by forcing failure along an identified failure plane.

Snow shear strength testing was conducted in two locations: in the vicinity of snow pit \# 1 and in the vehicle park (Figure 3.1). The first site was chosen because it enabled testing of snow layers that had been assessed for density and resistance via CPT, and the second site was used because it was easily accessible and allowed a large number of tests to be conducted across consistent snow layers in a relatively short period of time.

Shear strength testing typically requires a shear frame, generally with two to three vanes in order to maximise the entire frame surface area (Perla et al., 1982), plus a tensile measuring device such as a spring-balance or tensile meter. A shear frame with three vertical vanes (Figure 3.12), dimensions $200 \mathrm{~mm}$ x $200 \mathrm{~mm}$ x $50 \mathrm{~mm}$, with surface area of $0.04 \mathrm{~m}^{2}$, was manufactured in the Halley garage, consistent with the preferred size noted by Perla et al. (1982).

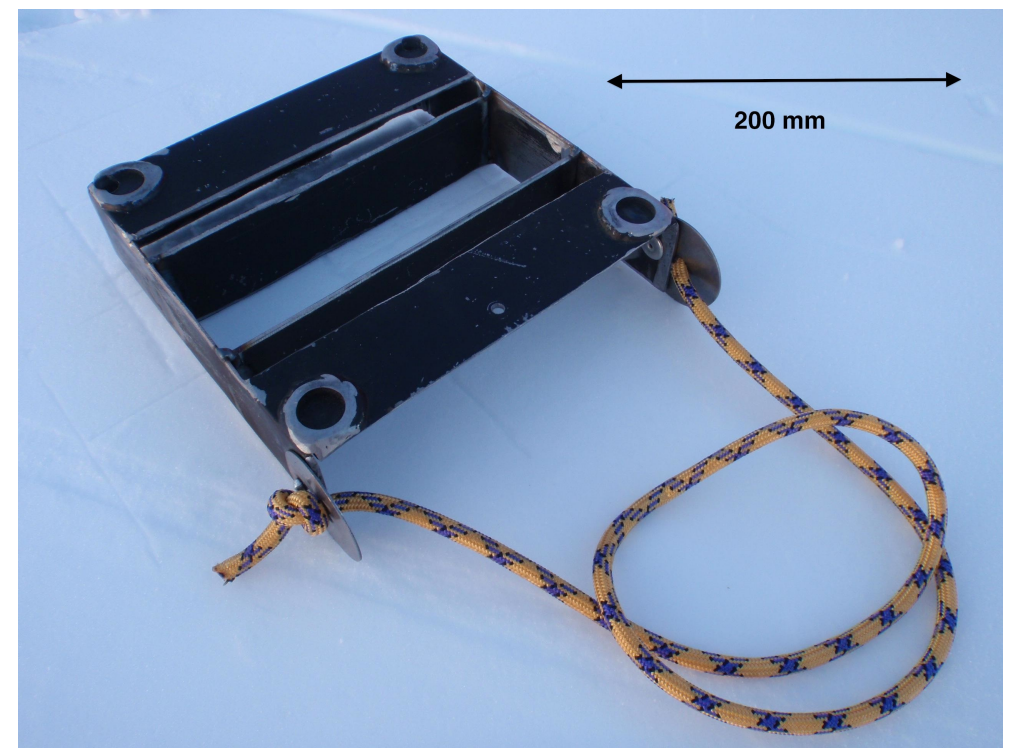

Figure 3.12: $0.04 \mathrm{~m}^{2}$ shear frame manufactured at Halley Station.

Numerous spring gauges at hand were trialled to test their suitability. However, all were limited in capacity and were not able to measure the tensile-force, estimated to be in the hundreds of kilograms. Eventually a 25 tonne 'dynafor' tensile load cell was found to use in measurement of tensile force (Figure 3.13).

Although this cell had more than adequate capacity for the envisaged shear-strength 


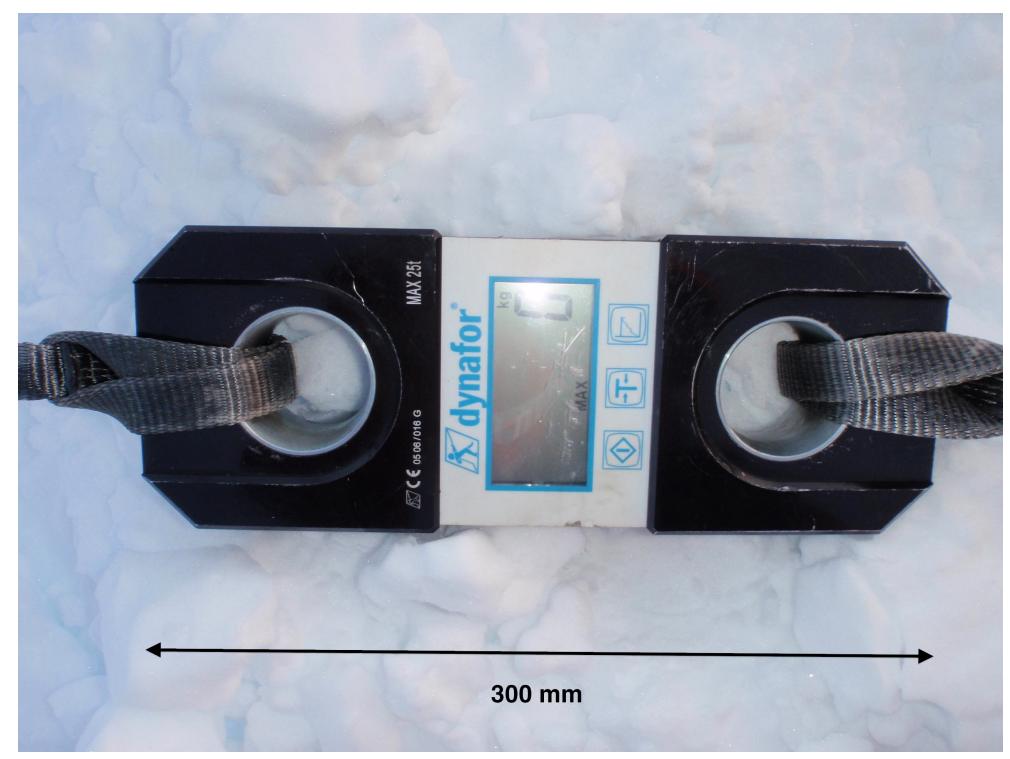

Figure 3.13: 25 tonne tensile cell used for shear strength testing.

testing its capacity meant that its accuracy was limited to $+/-10 \mathrm{~kg}$. Although not ideal, this device allowed shear strength testing of 'hard' snow layers. In order to better utilise the capacity of the available $25 \mathrm{t}$ load cell a number of trial tests were conducted using a large $800 \mathrm{~mm}$ x $800 \mathrm{~mm}$ x $50 \mathrm{~mm}$ pressed-metal wall-cladding panel. However, owing to the size of the panel and the lack of internal 'vanes' excessive 'skipping' occurred and this technique was not pursued further.

A tractor was required to transfer sufficient force to the shear frame, hence the alignment of the shear-strength testing was generally constrained by the nature of the surrounding snow surface (to ensure a predominantly horizontal loading) and the accessibility of the tractor to the site, adjacent to a deep snow pit. Figure 3.14 shows a typical test set-up. The snow to be tested was leveled using a snow saw, usually in a manner such that a number of tests of the same snow layer could be made adjacent to one another, perpendicular to the direction of pull; testing only occurred to a maximum depth of $\sim 100 \mathrm{~mm}$.

The shear frame was initially pushed into the snow using body-weight. However, this method of insertion typically caused the snow sample to fracture, thus for ensuing tests the snow saw was used to cut approximately $5 \mathrm{~mm}$ wide grooves in the snow which would accommodate the perimeter of the shear frame plus the vanes.

Once the shear frame was flush with the snow surface and positioned horizontally the frame was then connected via the load cell to the tractor using a series of slings. The tractor was then driven at a rate of approximately $10 \mathrm{~mm} \mathrm{~s}^{-1}$ until failure of the sample occurred, whereupon the load cell (which recorded the maximum load imparted) was inspected.

Initial tests were hampered by the shear frame 'skipping' over the snow surface once load was applied. This was in part because of the hardness of the snow being tested, and may also have occurred because of non-horizontal loading. This problem 


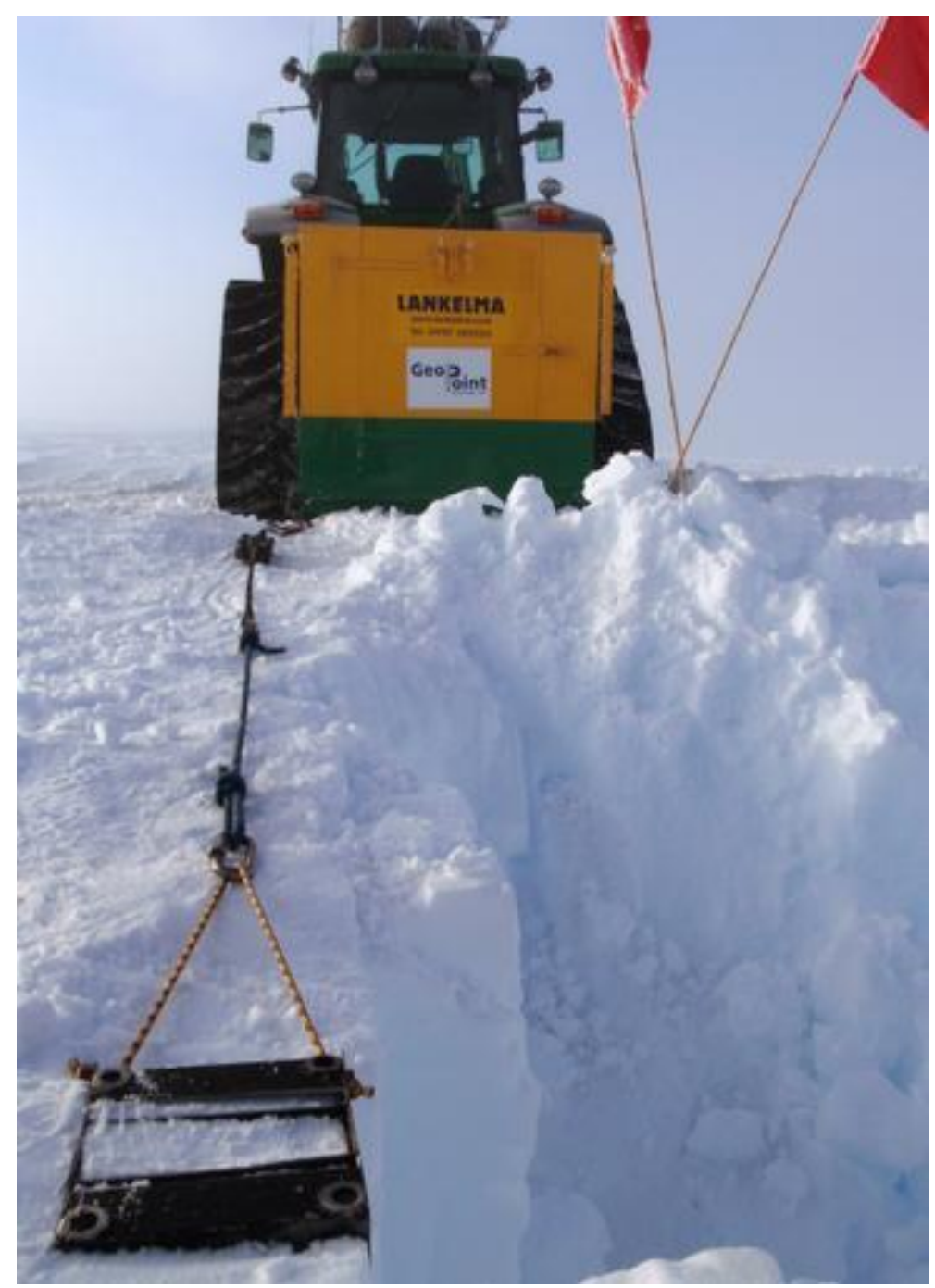

Figure 3.14: Typical shear test set-up shows tractor about to load shear frame inserted into surface snow layer, adjacent to snow pit \#1.

was partly resolved once additional vanes were constructed within the shear frame, although during later testing in the vehicle park, substantial 'skipping' still occurred. This was only countered by applying a substantial normal force (in the form of a $95 \mathrm{~kg}$ person) on top of the frame during testing. Insertion of the frame into the snow prior to a test often caused fracturing of the snow, thus rendering a test invalid, and even when no apparent disturbance occurred, some fracture of the snow within the shear frame may have occurred. Additional problems included the inaccuracy of the tensile gauge $(+/-10 \mathrm{~kg}=+/-2.5 \mathrm{kPa})$, incomplete shearing of samples, inaccuracy of density measurement, decrease in area owing to pre-cutting for insertion, and shear frame not leveled because of possible fracture upon loading.

The shear frame test is a relatively simple test that should provide a good measure of snow shear strength. Difficulties with the hardness of the snow to be tested and limited dedicated equipment meant that good quality shear-strength testing was not always possible; it appears difficult to conduct accurate shear-strength tests in hard, dense polar snow. However, a substantial number of shear strength tests were performed 
providing some basis for CPT comparison.

\subsection{Pressure Bulb Testing}

Although the primary aim of my research was to develop a method of estimating snow strength via in situ means, an application of this was the estimation of surface bearing capacity once layer strength has been established.

An estimate for surface bearing capacity can only be calculated once the penetration into the snow pack of the 'pressure bulb' beneath a surface load has been established. The purpose of my pressure bulb testing was therefore to verify Wuori's work (Wuori, 1962) and provide a local assessment of pressure bulb penetration, to assist in establishing surface bearing capacities. Some additional testing was also conducted in an attempt to verify the existence of a pressure bulb moving ahead of the cone or flat plate during CPT.

Pressure bulb testing was conducted at numerous locations as indicated in Figure 3.5. The testing site was typically chosen to provide convenient access, as the John Deere 7820 tracked tractor was typically used as a surface load. A number of opportunistic pressure bulb tests were also made when modules for the Halley Station were moved. In these cases a pit was quickly dug adjacent to the envisaged track of the module's skis to allow insertion of the pressure bulb underneath the centre of the module ski. Figure 3.15 shows an example of such an opportunistic test site.

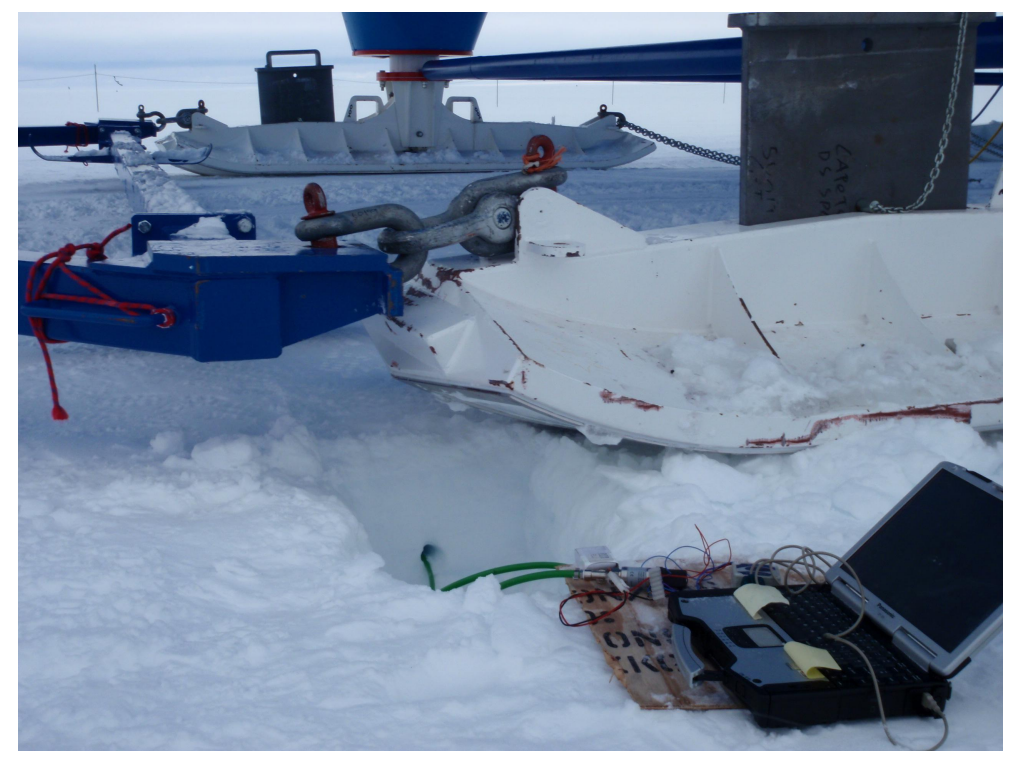

Figure 3.15: Opportunistic pressure bulb testing was conducted during module moves.

Limited resources precluded the use of expensive purpose-built pressure sensors, hence simple pressure sensors were made based on the experience of Turner et al. (2001) who used similar sensors for measuring peak sub-surface loads in soil under agricultural equipment. Each sensor (see Figure 3.16) consisted of a rubber pipettebulb connected to a Druck PTX vented gauge pressure transmitter by approximately 
$1.5 \mathrm{~m}$ of flexible hose. Two pressure transmitters were used, one rated to $25 \mathrm{kPa}$ and one to $100 \mathrm{kPa}$. Both transmitters were rated to a temperature of $-20^{\circ} \mathrm{C}$ and are accurate to $0.15 \%$. Each transmitter operates by converting a fluid pressure on a membrane to a voltage output. Hydraulic fluid (Falcon Lubricants, Hawk S32) was used as the pressure transmitting medium within each sensor.

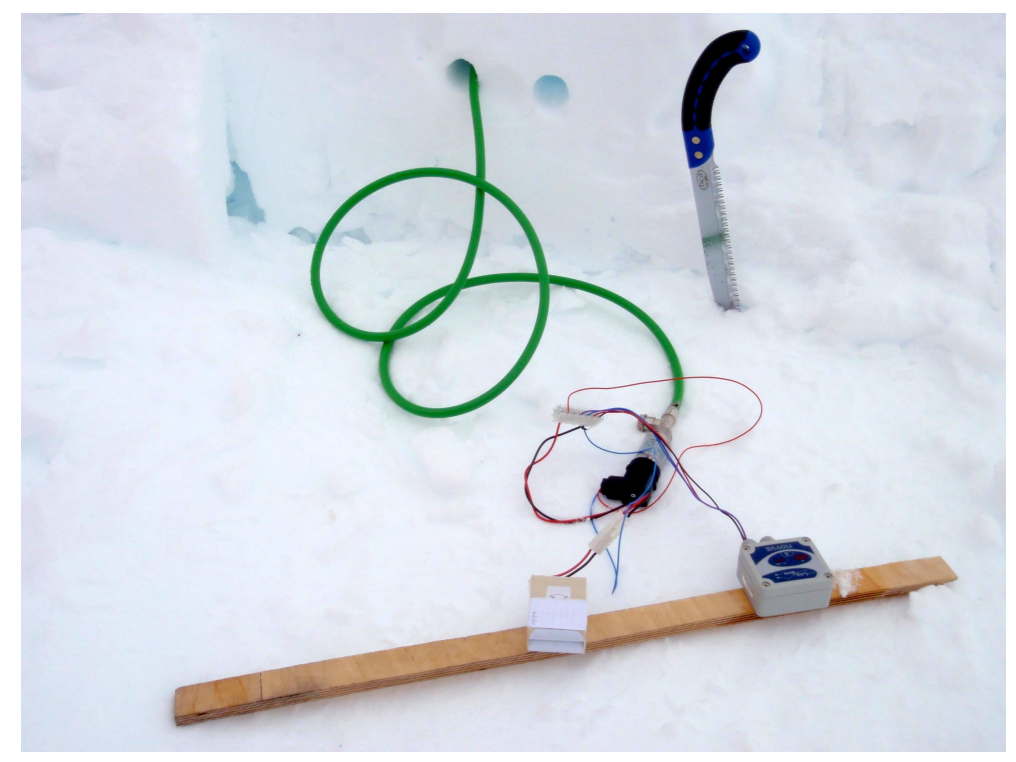

Figure 3.16: Pressure testing setup shows battery, Omni data logger and Druck PTX pressure transmitter. The rubber pipette-bulb is inside the hole.

Simple Omni LogBox-AA electronic data loggers were used for data acquisition, and each transmitter and logger was powered by rechargeable $6 \mathrm{~V}$ batteries. LogChart II Software Version 2.27 was used for data logger configuration and data recording. A simple schematic of the pressure bulb system is shown in Figure 3.17.

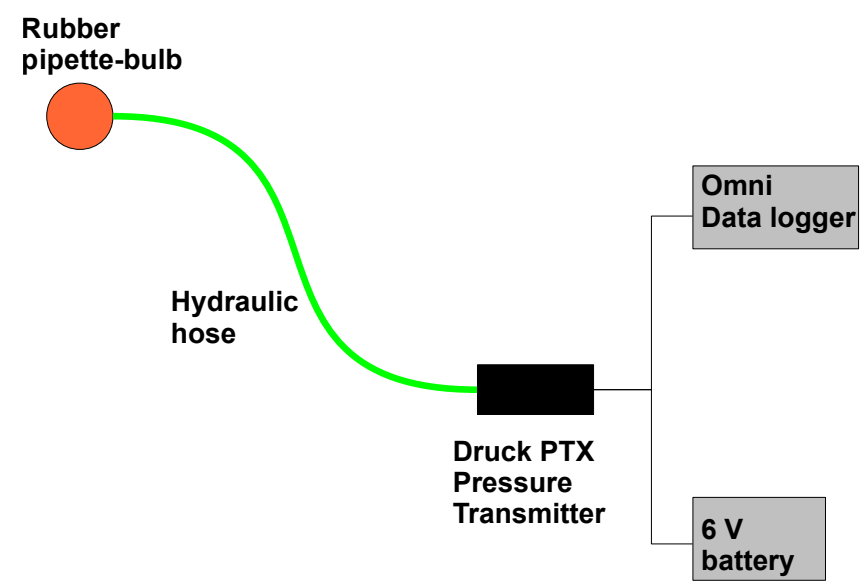

Figure 3.17: Rubber pipette-bulb filled with hydraulic fluid is connected to a Druck PTX pressure transmitter; power is provided by the $6 \mathrm{~V}$ rechargeable battery and data recorded by the Omni data logger. 
Calibration of each sensor was conducted by placing a series of metal washers of known weight onto each pressure bulb and registering the resulting increase in voltage via the LogBox-AA data logger. Each sensor was calibrated inside at a temperature of $\sim 15^{\circ} \mathrm{C}$ in an attempt to verify the linearity of the pressure response before field use, then additional calibration was conducted outside at temperatures of $\sim-10^{\circ} \mathrm{C}$ in order to reduce erroneous pressure fluctuations due to temperature and thus fluid volume change. A typical calibration curve with exponential line of best fit is shown in Figure 3.18.

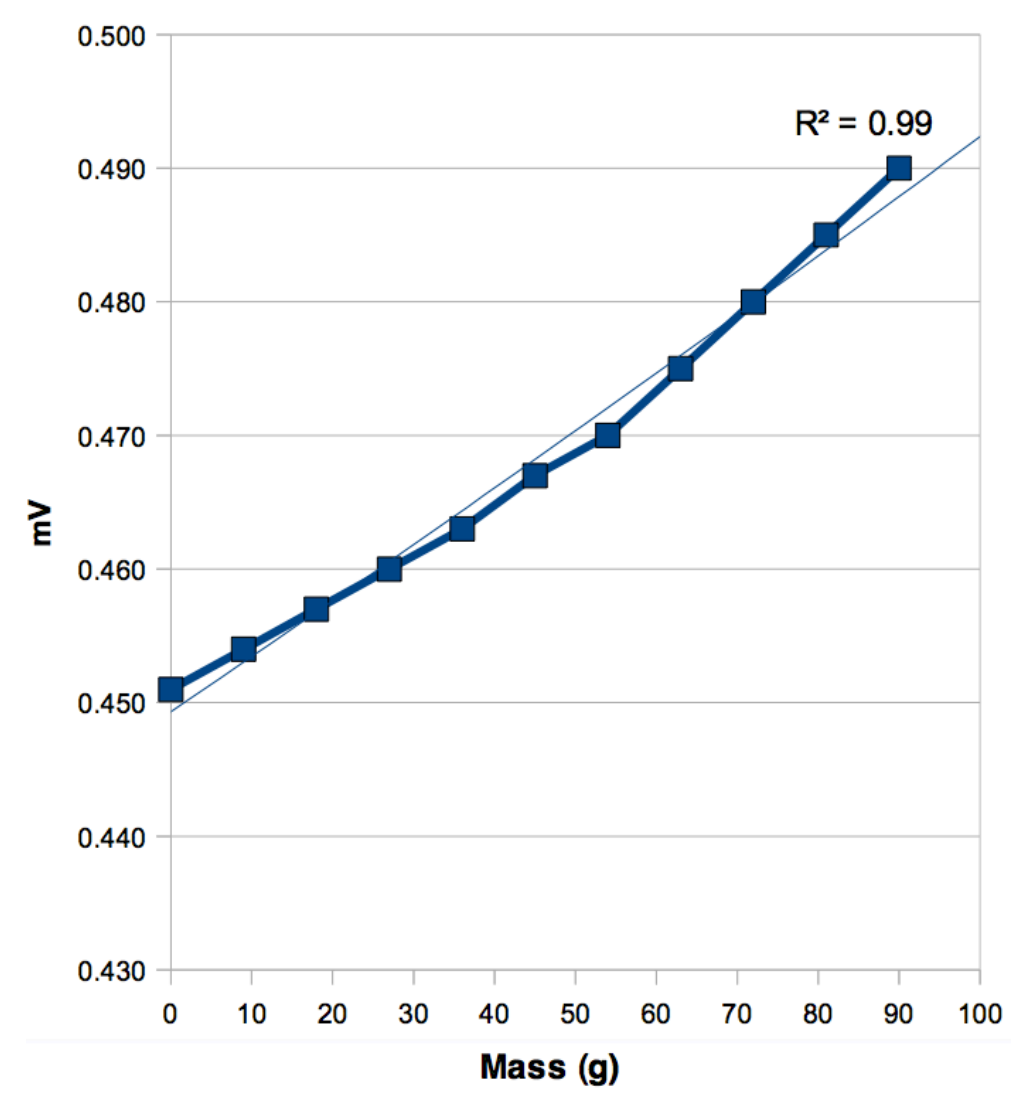

Figure 3.18: Typical calibration curve for pressure sensor with exponential line of best fit shows increase in $\mathrm{mV}$ output with mass.

Prior to each testing session a pit approximately $0.5 \mathrm{~m}$ deep was dug adjacent to the testing location to enable the pressure sensor to be inserted, typically at depths between $0.1 \mathrm{~m}$ and $0.5 \mathrm{~m}$. A $32 \mathrm{~mm}$ auger drill bit (the same size as the external dimensions of the pipette-bulb) was then used to drill horizontally into the snow to allow insertion of the pipette-bulb and hose, and the density of the snow layers above the pipette-bulb was measured gravimetrically.

A $30 \mathrm{~mm}$ external diameter rigid plastic pipe of $1 \mathrm{~m}$ length was then used to gently insert the pipette-bulb sufficiently $(\sim 1 \mathrm{~m})$ into the drilled hole to ensure the access pit did not collapse as the tractor was driven over the bulb. Once the pipette-bulb had been inserted it was typically left for up to an hour in order for temperatures to equilibrate and to dissipate any pressure established during the insertion process. The output was periodically monitored to ensure a constant base line. 
In conducting a test, typically logging commenced, a base line was established, and then the vehicle or load was manoeuvred to load the snow pack above the sensor. Upon removal of the load a base line was then re-established and then logging stopped. Because of the compaction of the snow pack only one measurement at any one discrete depth could ever be made at any one location. However, if access allowed, four different tests could be made at different depths (say $0.1 \mathrm{~m}, 0.2 \mathrm{~m}, 0.3 \mathrm{~m}$ and 0.4 $\mathrm{m}$ ) in the four sides of a central snow pit. The sides of the snow pits excavated for density-assessment purposes also provided suitable 'cuttings' in the snow from which the pressure test equipment could be inserted at various depths, but care had to be taken when manoeuvring heavy equipment next to such large pits.

When the pressure-sensing equipment was used in conjunction with the CPT, the pipette-bulb etc. was inserted in the usual manner and then the CPT equipment was manoeuvred over the bulb so that the cone or flat plate when driven to depth was directly over the pipette-bulb. However, such a method was of course prone to inaccuracy. An image depicting such an attempted assessment is shown in Figure 3.19.

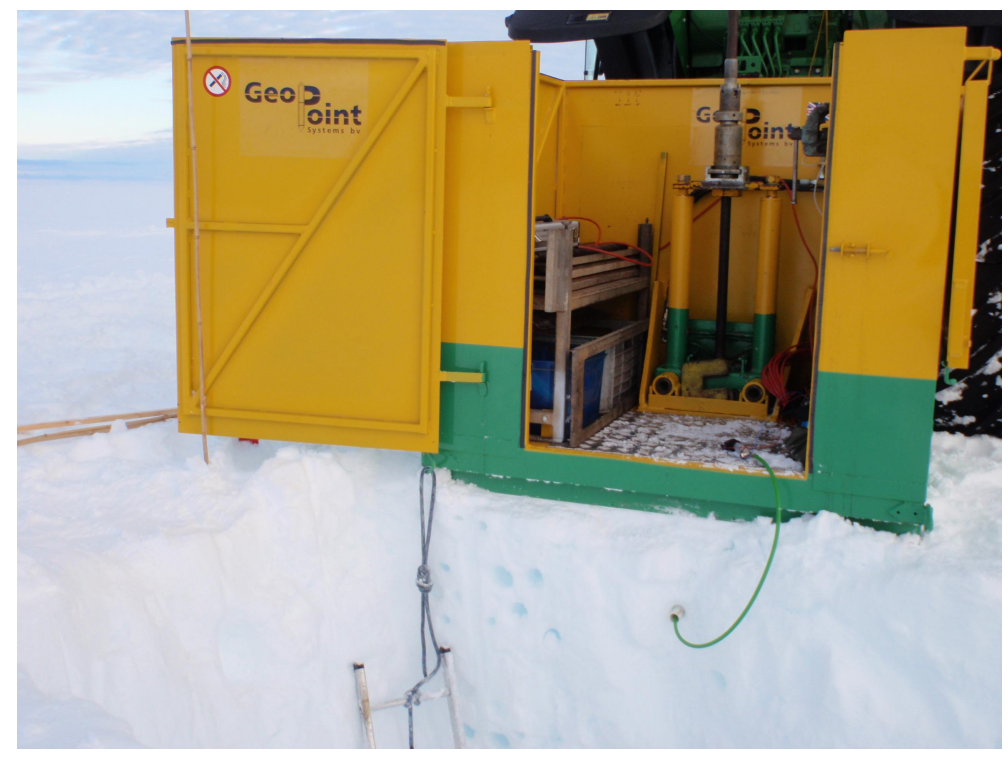

Figure 3.19: Setup for investigating pressure-bulb beneath cone or flat plate. The rubber pipette-bulb was inserted into the snow in a location estimated to be directly beneath the path of the cone before CPT was then conducted.

Numerous problems were encountered in using the pressure sensors. Essentially they appeared not accurate enough to record pressure changes. Although calibration suggested that a linear response could be achieved, actual load data proved erratic. Contraction or expansion of the pipette-bulb with minor pressure fluctuations also meant that the system was not always devoid of air, with a 'floppy' bulb often resulting. Further, the elasticity of the rubber pipette-bulb at such temperatures is not known hence minor pressure fluctuations may not have been recorded. Other problems encountered included lag in response, susceptibility to temperature variations, disturbance upon installation and the inability to conduct more than one test in any 
layer.

Simple yet innovative pressure sensors, of a design previously proven in soil were manufactured and used in an attempt to assess the extent of the pressure-bulb beneath both surface loads and penetrating cones/plates in snow. Although the sensors and the logging equipment proved very robust and easy to deploy the results obtained were influenced by temperature and installation and alternative pressure measuring sensors would be employed in the future should resources allow.

\subsection{Ground Penetrating Radar (GPR)}

Radio Echo Sounding (RES) of ice masses has been performed by the British Antarctic Survey and Scott Polar Research Institute (SPRI) since 1962 (Swithinbank, 1969), and SPRI in conjunction with the U.S. National Science Foundation (NSF) and the Technical University of Denmark performed extensive aerial RES coverage of Antarctica in the late 1960s and into the 1970s (Bingham and Siegert, 2007); Rees (2006) provides a comprehensive guide to remote sensing of the cryosphere. More recently, land-based Ground Penetrating Radars (GPR) of various frequencies have become increasingly popular in snow science and have been used to investigate the spatial extent of snowhorizons, detect crevasses, assess bedrock depth and numerous other applications (see Arcone et al. (2004), Marshall et al. (2007) etc.). However, their use as yet has not been refined enough to enable the determination of snow microstructural properties. Even a 4 - $6 \mathrm{GHz}$ FMCW radar may only have a resolution of $\sim 6 \mathrm{~cm}$ (email communication S.Arcone, 27 June, 2008), and because penetration is limited at such high wavelengths, compromises must often be made when choosing an appropriate radar frequency. GPR was used throughout my testing, to validate its use as a means of spatially extending data derived from point cone penetration tests, thus allowing extrapolation of derived snow mechanical properties over a wide spatial area.

Testing was performed at numerous locations about the Halley V site, some of which are noted on Figure 3.1. Additional testing was also conducted in the 'hinge' zone between shelf ice and sea ice some $20 \mathrm{~km}$ distant from the base in an attempt to verify crevasse location for the establishment of a safe route of passage. This testing is not examined within this thesis but was performed to obtain additional GPR familiarity.

The GPR equipment used was a Geophysical Survey Systems, Inc. (GSSI) TerraSIRch SIR System-3000 (SIR-3000) and a GSSI 400 MHz (centre-frequency) ground coupled antenna. The technical specifications of the SIR-3000 are noted in Table 3.3 and the $400 \mathrm{MHz}$ antennae specifications are noted in Table 3.4.

The GPR was generally used in 'distance' mode with the GSSI Model 620 (16 " survey wheel) mounted to the rear of the antennae, with the antennae typically towed behind a skidoo. On occasion it was used in 'time' mode without the survey wheel and was also sometimes towed by foot or tractor, depending on the terrain and/or 
Table 3.3: GSSI TerraSIRch SIR System-3000 specifications.

$\begin{array}{ll}\text { Specification } & \text { Value } \\ \text { Internal Memory } & 1 \mathrm{~GB} \\ \text { CPU } & \text { Intel RISC 206 MHz } \\ \text { Display } & 8.4 " \mathrm{TFT}, 800 \times 600 \\ \text { Dimensions } & 31.5 \times 22 \times 10.5 \mathrm{~cm} \\ \text { Weight } & 4.1 \mathrm{~kg} \\ \text { Operating Range } & -10 \mathrm{to} 40^{\circ} \mathrm{C} \\ \text { Transmission Rate } & \leq 100 \mathrm{kHz} \\ \text { Data Acquisition } & 220 \mathrm{scans} / \mathrm{s} @ 256 \text { samples / scan } \\ & 120 \mathrm{scans} / \mathrm{s} @ 512 \text { samples / scan } \\ \text { Resolution } & 8 \mathrm{bit} \text { to } 16 \mathrm{bit} \\ \text { Samples per scan } & 256,512,1024,2048,4096,8192 \\ \text { Time Range } & 5-8000 \mathrm{~ns} \\ \text { Gain } & 1-5 \text { points automatic / manual }\end{array}$

Table 3.4: $400 \mathrm{MHz}$ Antennae specifications.

$\begin{array}{ll}\text { Specification } & \text { Value } \\ \text { Pulse Length } & 2.5 \mathrm{~ns} \\ \text { Dimensions } & 30 \times 30 \times 17 \mathrm{~cm} \\ \text { Weight } & 6.4 \mathrm{~kg} \\ \text { Penetration Depth } & \sim 5 \mathrm{~m} @ \epsilon=9\end{array}$

application. A typical survey setup is shown in Figure 3.20.

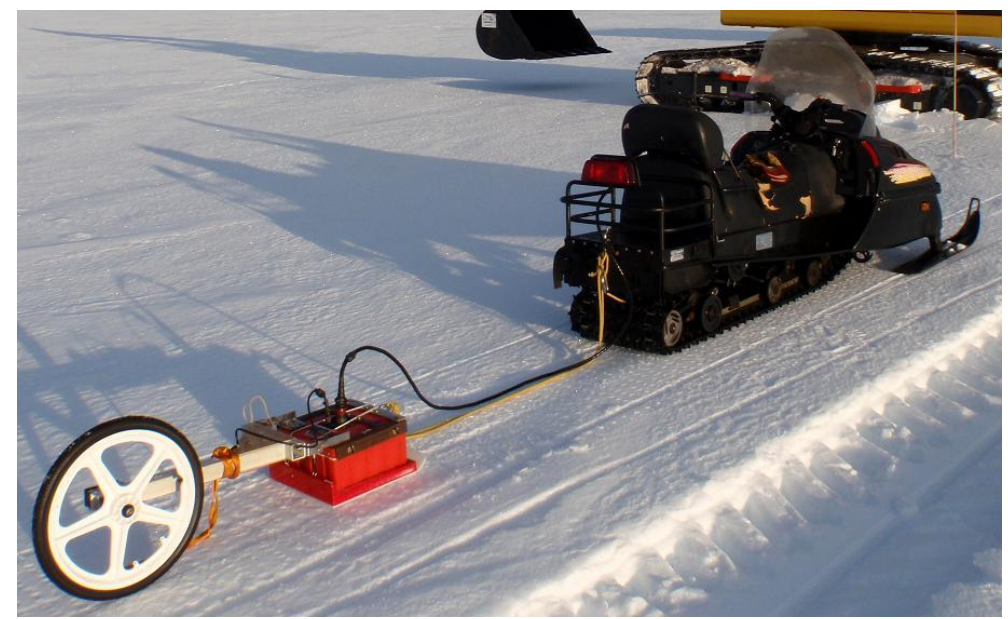

Figure 3.20: The GPR was towed behind a skidoo and operated in 'distance' mode.

Prior to each test (or session of testing) the GPR needed to be calibrated for both horizontal distance measured and for depth of penetration. Procedures for this are detailed within the SIR-3000 User's Manual (Geophysical Survey Systems Inc., 2006) but the general procedure is outlined briefly below.

Depth calibration must be performed by operating the GPR over an object (such as a shovel) buried at a known depth. Calibration of the depth returned by the GPR with the known depth generates an average dielectric constant for the material that 
can be used for subsequent testing. This dielectric constant also suggests an average density of the snow (to object depth) in accordance with the equation suggested by Kovacs et al. (1995). Modification of the dielectric constant can be used in subsequent testing if the average density of the snow (to desired penetration depth) is known or supposed to have changed.

Distance calibration is simply performed by dragging the radar over a known distance (preferably $50 \mathrm{~m}$ ) during which the number of 'ticks' generated is recorded. Repetition of this test allows an average number of 'ticks generated per metre traveled' to be established, essentially calibrating the distance assumed traveled by the unit. Typically, when using the SIR-3000 it is recommended that the gain is automatically set (using procedures detailed within the manual (Geophysical Survey Systems Inc., 2006)) and then set to 'manual' so that the gain values then remain constant throughout subsequent testing.

The area or transect to be covered was examined to identify obvious geographical hazards or restrictions, and then the SIR-3000 was assembled and mounted to the appropriate tow vehicle. Calibration (as described above) was performed if necessary, and menu settings within the SIR-3000 operating programme were manipulated for the subsequent testing. Typical settings are in Table 3.5. Further information on these settings can be obtained within the SIR-3000 Manual (Geophysical Survey Systems Inc., 2006).

\begin{tabular}{|c|c|}
\hline Parameter & Value \\
\hline Transmission Rate & $400 \mathrm{MHz}$ \\
\hline Mode & Distance \\
\hline GPS & Custom \\
\hline Format & 16 \\
\hline Range & 50 \\
\hline Diel & 1.9 \\
\hline Rate & 120 \\
\hline Scn/Unit & 120 \\
\hline Gain & Auto (then Manual) \\
\hline Position & Auto \\
\hline Filters & Default Settings \\
\hline
\end{tabular}

A handheld Garmin ETrex Venture GPS was generally connected to the SIR-3000 prior to testing to allow the profile start and end positions to be recorded within the data header information. To collect data, 'Collect Run' mode is entered and then upon hearing a confirmatory audible 'beep', data collection can occur; when in 'distance' mode data will start being recorded once movement occurs. Upon completion of a profile, the data recording is stopped, then the data saved to the internal hard-drive of the SIR-3000. Upon completion of testing, data was downloaded from the SIR-3000 unit, and inspected and manipulated using GSSI's proprietary Software, RADAN 6.5 
(Geophysical Survey Systems Inc., 2007).

The SIR-3000 proved a robust and user-friendly piece of equipment. The only problems that were encountered occurred when performing profiles moving from seaice onto glacial ice. In this situation the ideal gain and dielectric constant for each medium is different and changing such parameters during a test impairs the ability to compare radar returns from each material. This problem was typically addressed by making two passes during such profiles, in opposite directions, with both gain and dielectric constant set for the initial medium, thence held constant throughout the profile.

GPR profiling of both the Halley $\mathrm{V}$ and Halley VI site was conducted in order to verify the facility of the tool in complementing the data provided through CPT. The GSSI SIR-3000 system was used in various manners, proving versatile, robust and effective.

\subsection{Summary}

This chapter has summarised all the methods employed in obtaining data for this research, including the methods used in successfully conducting the first CPT in Antarctica. CPT equipment and methods for employment have been proven over many years in the geotechnical engineering field, hence reliable practices could be drawn upon. Some of the problems encountered, and some of the specific issues under investigation were also considered. The CPT and associated equipment worked reliably and consistently and considerable testing was conducted to address many of the questions outlined earlier. The next chapter presents results from the pilot study penetrative testing in Greenland. 


\section{Chapter 4}

\section{Results \& Analysis (A) - Fugro mini-penetrometer}

A pilot study of shallow penetrative testing in polar snow was carried-out upon the Greenland ice cap in April 2009 using a hand-driven mini-penetrometer. Fifty-three tests (see Table 4.1) were conducted in an attempt to address some of the questions noted in Chapter 3 and to assist in drawing conclusions from the primary CPT in Antarctica, discussed in the next chapter.

Table 4.1: Fugro mini penetrometer testing.

\begin{tabular}{|c|c|c|c|c|}
\hline Test & Site & 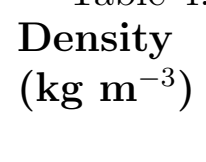 & $\begin{array}{l}\text { Insertion } \\
\text { Rate } \\
\left(\mathrm{mm} \mathrm{s}^{-1}\right)\end{array}$ & Remarks \\
\hline $1-9$ & 1 & 450 & 100 & 2-day old fine grained wind slab. \\
\hline $10-16$ & 1 & 450 & 20 & 2-day old fine grained wind slab. \\
\hline $17-25$ & 2 & 450 & 100 & $\begin{array}{l}\text { Repeat of Tests } 1-9 . \text { Weak layer } \sim 20 \\
-22 \mathrm{~cm} .\end{array}$ \\
\hline $26-33$ & 2 & 450 & 20 & Weak layer $\sim 20-22 \mathrm{~cm}$ \\
\hline $34-36$ & 2 & 286 & 50 & Horizontal test. Erratic rate. \\
\hline $37-39$ & 3 & 286 & 50 & Partially pre-fractured. \\
\hline 40 & 3 & 286 & 100 & Examining variation in rate. \\
\hline 41 & 3 & 286 & 50 & Examining variation in rate. \\
\hline 42 & 3 & 286 & 20 & Examining variation in rate. \\
\hline 43 & 3 & 286 & 50 & Examining spatial consistency. \\
\hline 44 & 3 & 286 & 50 & Examining spatial consistency. \\
\hline 45 & 3 & 286 & 50 & Examining spatial consistency. \\
\hline 46 & 3 & 286 & $\mathrm{~N} / \mathrm{A}$ & Temperature calibration test. \\
\hline $47-52$ & 4 & 450 & 50 & $\begin{array}{l}\text { Six tests in wind pack. Hard layer } \\
22 \mathrm{~cm} .\end{array}$ \\
\hline 53 & 2 & 450 & 50 & $\begin{array}{l}\text { Examining 'chipping' in unconfined } \\
\text { block. }\end{array}$ \\
\hline
\end{tabular}

A number of these tests were conducted using the penetrometer whilst not recording in an attempt to further investigate cone effective area, the formation of a compacted zone, and the brittle fracture of unconfined snow. All tests were conducted between 
the 4th and 5th April 2009, with air temperature varying between $-6^{\circ} \mathrm{C}$ and $-18^{\circ} \mathrm{C}$, and snow temperature varying between $-13^{\circ} \mathrm{C}$ and $-23^{\circ} \mathrm{C}$. Rates of penetration are approximate and based on manual time measurement during probe insertion, and each test was to a depth of $50 \mathrm{~cm}$ unless terminated earlier because of impenetrable layers.

\subsection{Distance for resistance to be mobilised}

Examination of Figure 4.1 depicting nine continuous tests of penetration and extraction through a homogeneous snow layer (Tests 1 to 9) suggests that in each test, a finite distance must be covered before increasing cone resistance trends towards a probable steady-state which may be truly representative of the resistance offered by the snow.

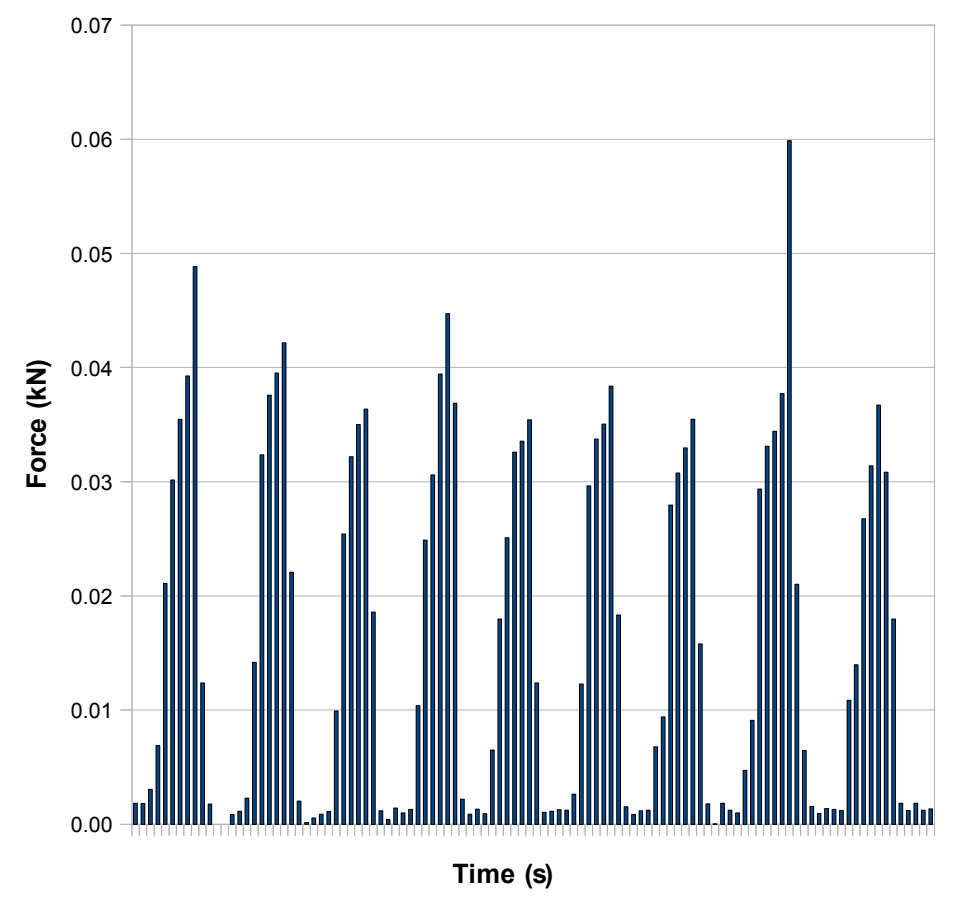

Figure 4.1: Nine penetration cycles (insertion/extraction) in homogeneous snow suggest that tip resistance tends towards a steady-state.

This inclination for tip resistance to rise towards a pseudo-plateau in homogeneous snow can also be observed in Figure 4.9 (discussed further shortly) and is also evident through examination of horizontal test data for Greenland (not shown). Although based on very limited data, Figure 4.2 (Tests 43 and 44) perhaps presents this better.

These tests were in less dense snow where peak resistance would be expected to be reached earlier (in the push) and thus detection of a 'steady-state' resistance is possible.

The supposition that a certain depth of penetration must occur before resistance within a homogeneous layer is realised was suggested by De Beer (1963) who showed that a penetrating cone must reach a 'critical depth' within any (homogeneous) layer 


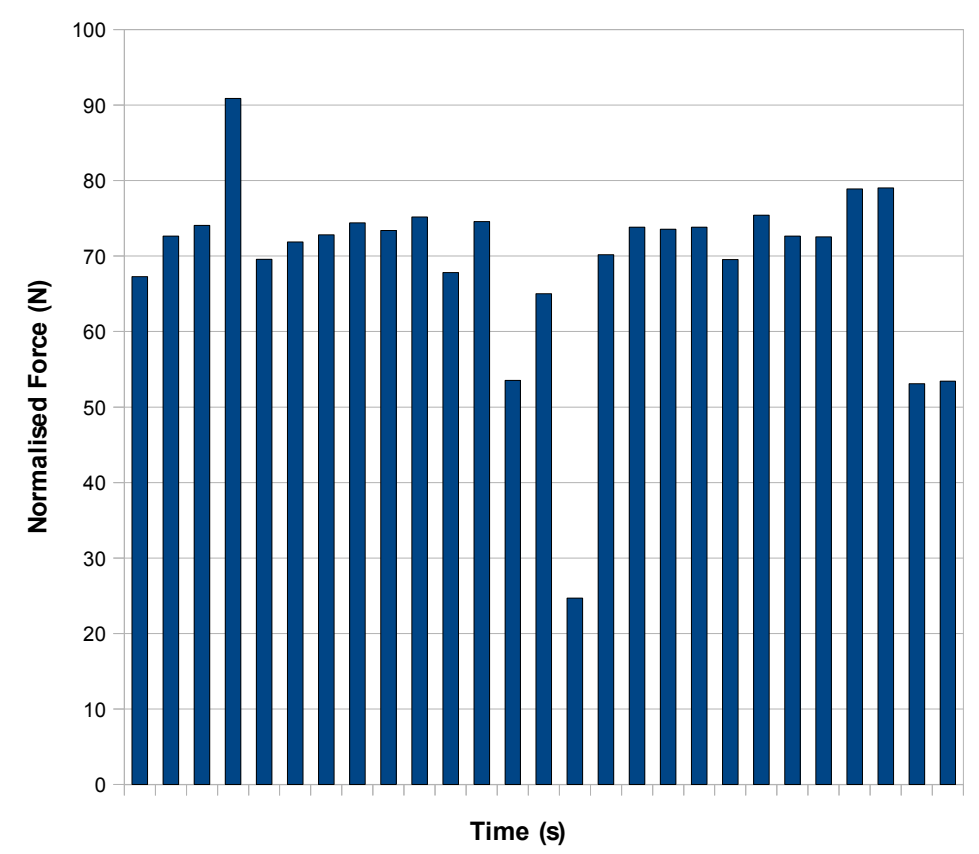

Figure 4.2: In less dense snow a representative steady state force of perhaps $70 \mathrm{~N}$ is reached sooner (two penetration cycles shown).

before the full point resistance within that layer is mobilised. In this testing it appears that a true steady-state resistance may have been demonstrated. This phenomenon is discussed in more detail with respect to the larger diameter CPT cone in the next chapter.

\subsection{Tip resistance \& sleeve friction}

Tests 1 to 9 were conducted in close proximity (yet still $>2.5$ cone radii apart as suggested by L. Gibson, December, 2008) as shown in Figure 4.3, and Figure 4.4 shows the time versus tip resistance trace for these tests, obtained using the minipenetrometer. Note that tip resistance has been offset, as initial calibration resulted in negative values at zero tip force; data recorded during penetrometer extraction is also shown.

Spatial consistency is evident as well as increasing resistance within this homogeneous snow layer. Variation of shaft friction with depth for tests 1 to 9 is presented in Figure 4.5. Note that these data also incorporate extraction of the penetrometer, evident from a time of approximately 8 seconds where sleeve friction becomes negative.

Although some inconsistency with time (depth) is evident between tests, possibly due to difficulties in ascertaining the start and finish of each test, the traces appear consistent and are not significantly different (via unpaired t test with $95 \%$ confidence interval). Both tip resistance and sleeve friction appear to increase during penetration into a homogeneous snow mass. These matters are discussed further in the next chapter. 


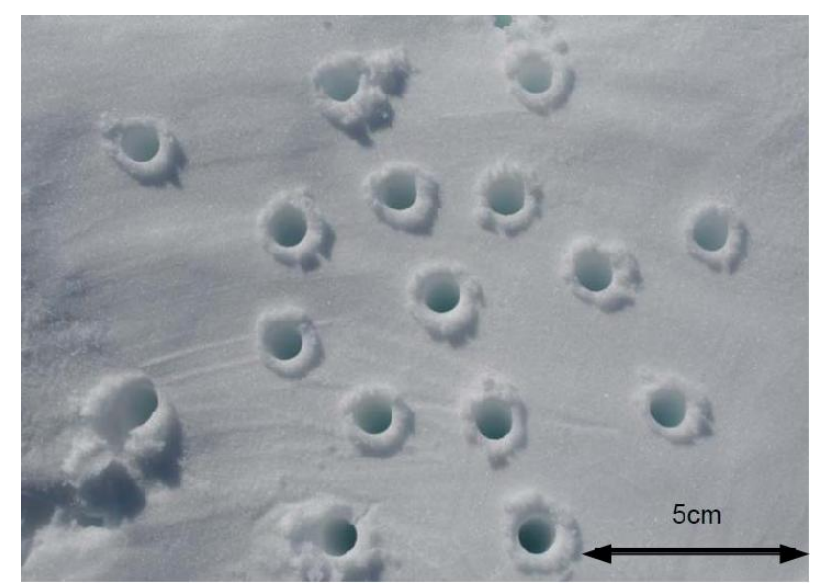

Figure 4.3: Spacing of Tests 1 to 9 (centre plus outer holes).

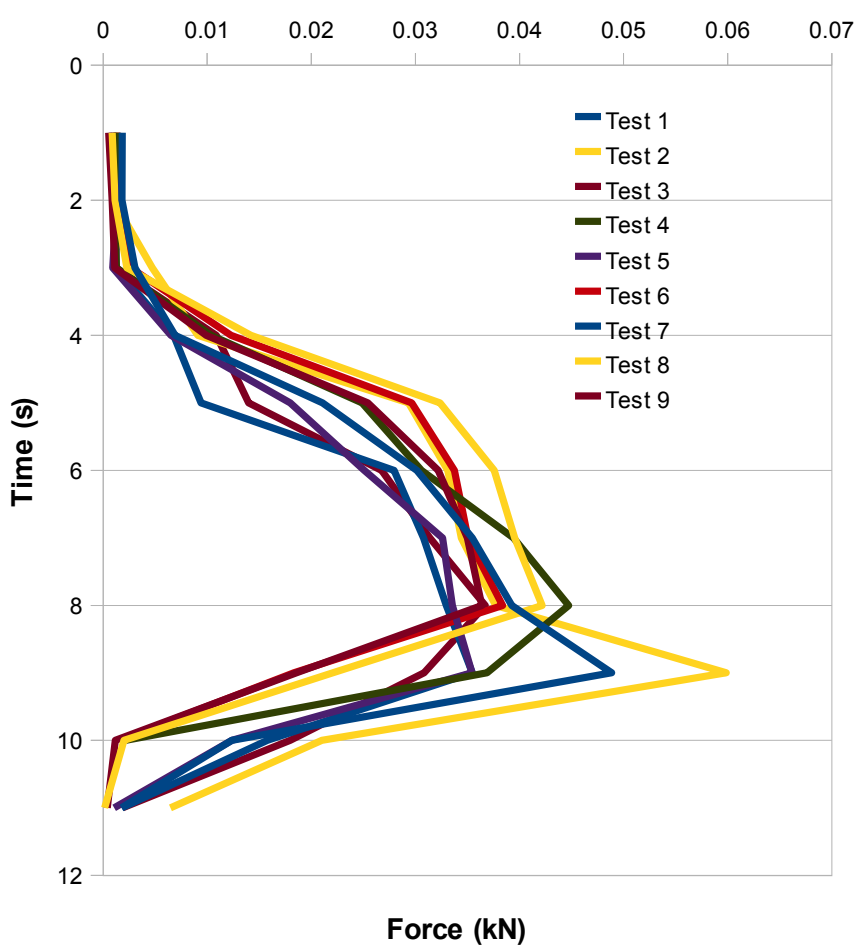

Figure 4.4: Cone Tip Resistance vs Time (depth) for Tests 1 to 9 (insertion and extraction) shows spatial consistency and increasing resistance with penetration.

\subsection{Rate Effects}

Insufficient testing was conducted with the hand-driven penetrometer to conclusively verify rate effects, however, some effect was evident. Mean tip resistance and mean sleeve friction for Tests 1 to $9(\mathrm{n}=108)$ at a rate of $100 \mathrm{~mm} \mathrm{~s}^{-1}$ and co-located Tests 10 to $16(\mathrm{n}=151)$ conducted at $20 \mathrm{~mm} \mathrm{~s}^{-1}$ (the inner-ring of holes, Figure 4.3$)$ are shown in Table 4.2. Mean values at different rates are statistically different (via unpaired t test with $95 \%$ confidence interval) so penetration rate is seen to have an effect on both tip resistance and sleeve friction.

Table 4.3 summarises the variation in average friction values across various tests, 


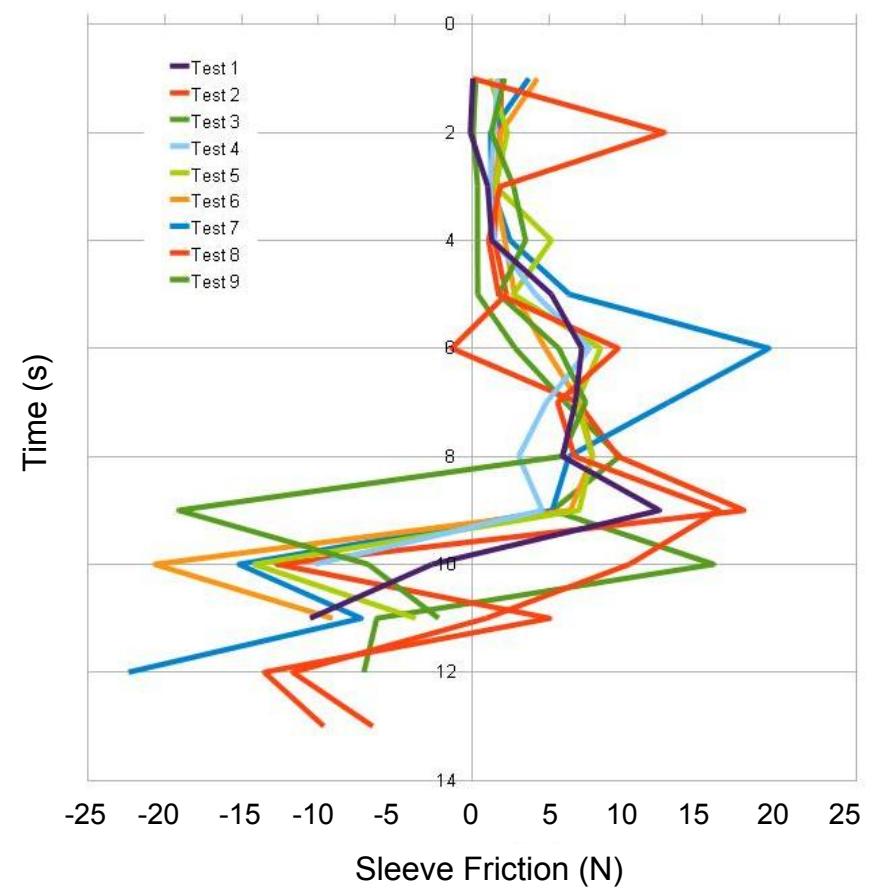

Figure 4.5: Sleeve friction vs time (depth) for Tests 1 to 9 (insertion and extraction) shows spatial consistency; negative values were recorded during extraction.

Table 4.2: Variation in tip resistance and sleeve friction with penetration rate.

$\begin{array}{lll}\text { Penetration } & \begin{array}{l}\text { Mean Tip Resistance } \\ \text { Rate }\left(\mathbf{m m ~ s}^{-1}\right)\end{array} & \begin{array}{l}\text { Mean Sleeve Friction } \\ (\mathbf{N})\end{array} \\ 20 \mathrm{~mm} \mathrm{~s}^{-1} & 22.93 & 77.0 \\ 100 \mathrm{~mm} \mathrm{~s}^{-1} & 15.55 & 73.7\end{array}$

at different penetration rates, within snow of two different densities. In this data the difference between mean $(\mathrm{n}>100)$ sleeve friction at the same rates is not statistically significant, whereas the difference between mean sleeve friction at different rates is statistically significant (via unpaired t test with $95 \%$ confidence intervals).

Table 4.3: Variation of mean sleeve friction with density.

\begin{tabular}{|c|c|c|c|}
\hline Tests & $\begin{array}{l}\text { Density } \\
\left(\mathrm{kg} \mathrm{m}^{-3}\right)\end{array}$ & $\begin{array}{l}\text { Rate } \\
\left(\mathrm{mm} \mathrm{s}^{-1}\right)\end{array}$ & $\begin{array}{l}\text { Mean Sleeve Friction } \\
(\mathrm{N})\end{array}$ \\
\hline $1-9$ & 450 & 100 & 88.06 \\
\hline $10-16$ & 450 & 20 & 126.59 \\
\hline $17-25$ & 450 & 100 & 99.07 \\
\hline $26-33$ & 450 & 20 & 137.60 \\
\hline $37-39$ & 286 & 50 & 71.55 \\
\hline
\end{tabular}

These data suggest that sleeve friction decreases with increased penetration rate, consistent with tip resistance data. This observation is contrary to many forms of friction, notably viscous friction where friction increases with velocity. However, it is consistent with data from Colbeck (1992) who reports that as the speed of miniature skis on snow increased from $3 \mathrm{~cm} \mathrm{~s}^{-1}$ to $5 \mathrm{~m} \mathrm{~s}^{-1}$ friction reduced greatly. This phenomenon is further discussed in the next chapter where dedicated rate-effect tests using 
CPT are discussed.

\subsection{Age hardening}

Consistent with the literature (e.g. Abele (1990), Blaisdell et al. (1998)), age-hardening of the snow was expected and was detected via use of the manual-driven mini-penetrometer, as shown in Figure 4.6.

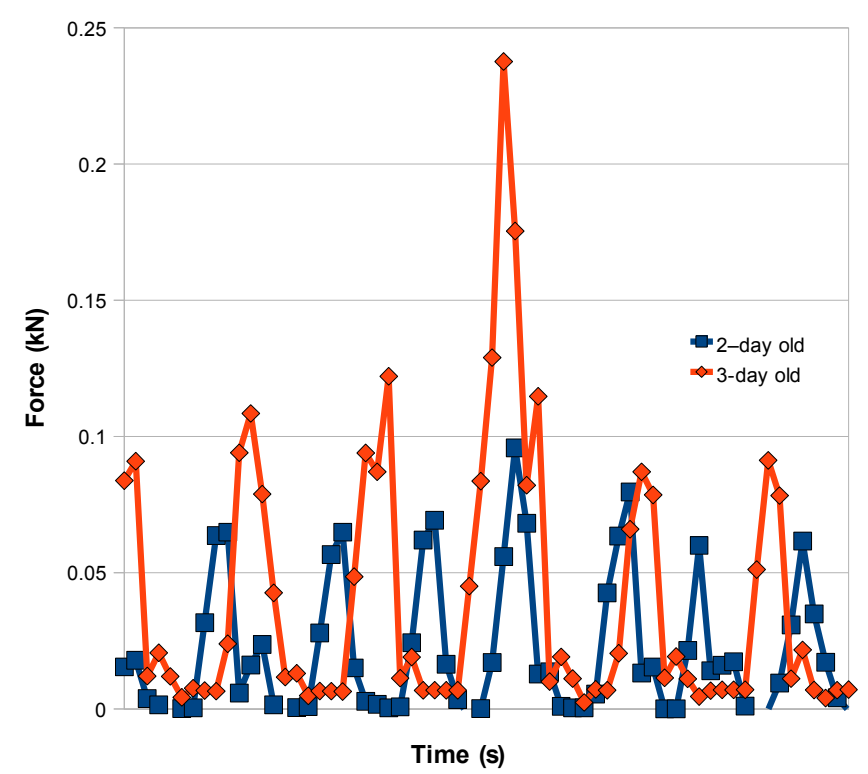

Figure 4.6: Tip resistance is generally greater in age-hardened snow. Seven penetration cycles are shown in the 2-day old snow and six penetration cycles are shown in the 3-day old snow.

This increase in hardness at the same location is caused by two primary processes: compaction, resulting in densification, and sintering, whereby the number and size of bonds between ice grains increases. Both of these mechanisms typically occur over time hence an increase in hardness of the snow was expected.

\subsection{Variation with density}

As shown in Table 4.1, penetrometer tests were conducted within homogeneous snow of limited different densities. Figure 4.7 shows the tip force recorded during tests 34 to 36 in snow of density $286 \mathrm{~kg} \mathrm{~m}^{-3}$ compared with the tip force recorded during tests 43 to 45 in snow of density $450 \mathrm{~kg} \mathrm{~m}^{-3}$. At the same penetration rate, differences in recorded force were observed. This is consistent with historical observations and suggests that ultimate cone tip resistance is higher for higher density snow.

Owing to the limited depth and diversity of snow conditions at the test site, only one series of tests involving penetration through stratified snow was undertaken. Figure 4.8 shows continuous data from Tests 47 - 52 showing insertion and extraction of the 


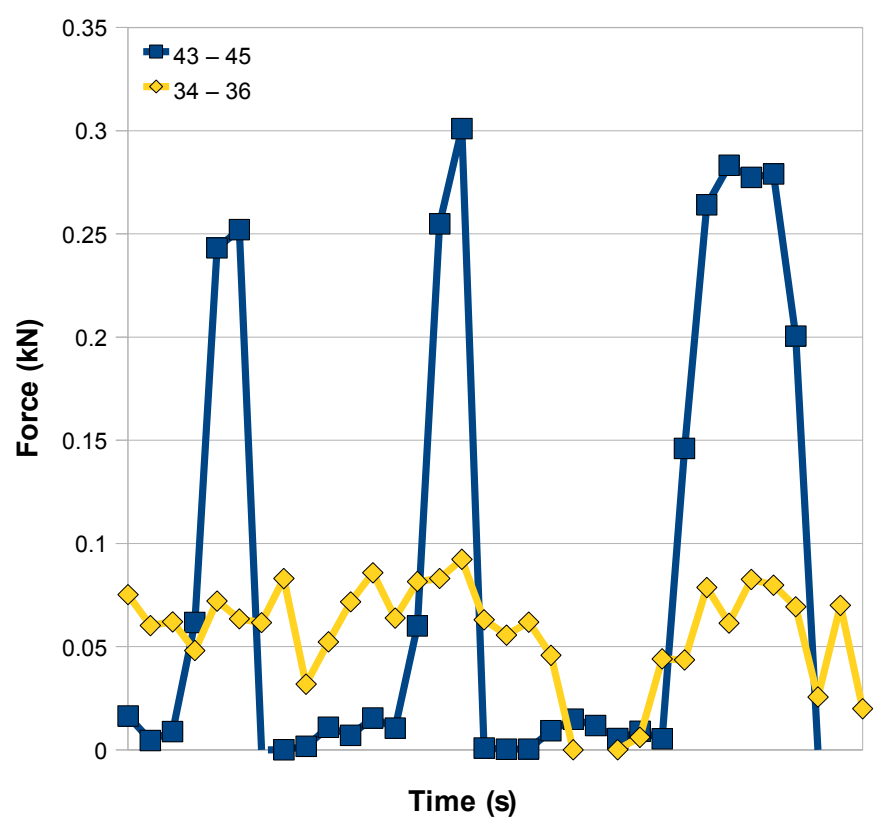

Figure 4.7: Force on the penetrometer tip is greater in higher density snow. Tests 34 - 36 were in snow of density $286 \mathrm{~kg} \mathrm{~m}^{-3}$ and tests 43 - 45 were in snow of density 450 $\mathrm{kg} \mathrm{m}^{-3}$. Three penetration cycles are shown.

penetrometer (recording was not stopped between tests). Stratigraphy consisted of bulk snow of density $450 \mathrm{~kg} \mathrm{~m}^{-3}$ dissected by a horizontal icy layer, density unknown.

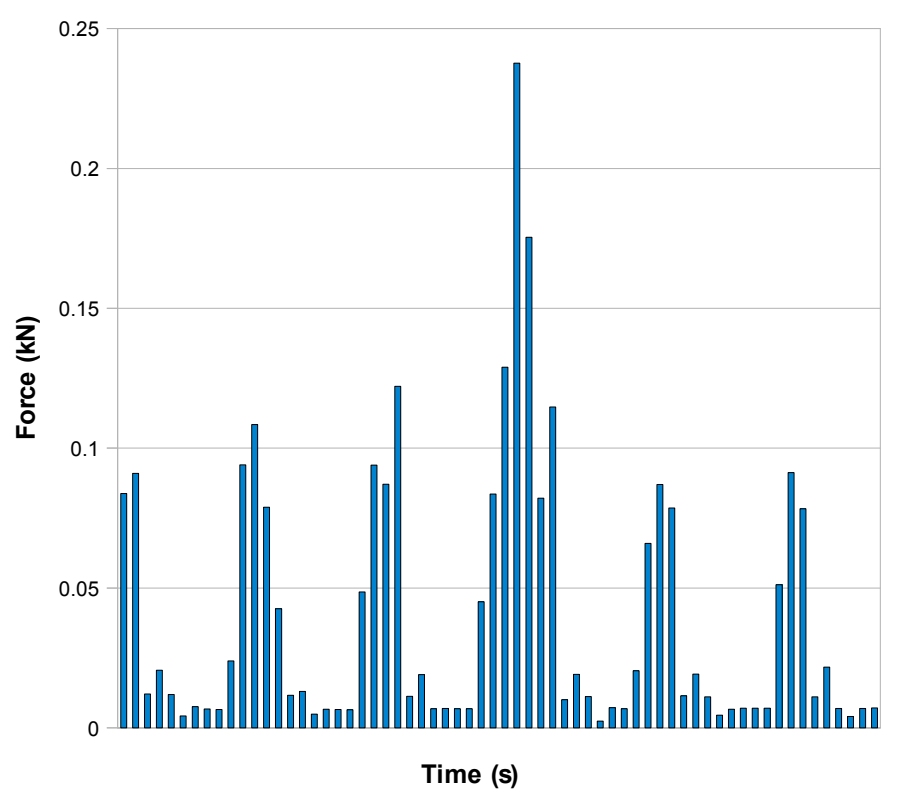

Figure 4.8: Tip resistance is shown to increase markedly upon penetration of a hard icy layer (density unknown). Six penetration cycles shown.

Although the limited sampling rate of the cone precludes a more accurate analysis, in each of these tests the tip resistance can be seen to initially increase before rising further, presumably upon striking the icy layer, then falling briefly to a sustained level, before dropping again as the each test is terminated. Although limited in nature, the 
testing described above suggests that cone tip resistance, as expected, is proportionate to snow density.

Figure 4.9 presenting data from Tests 1 - 9 shows that during penetration in homogeneous snow, sleeve friction tends to trend with tip resistance.

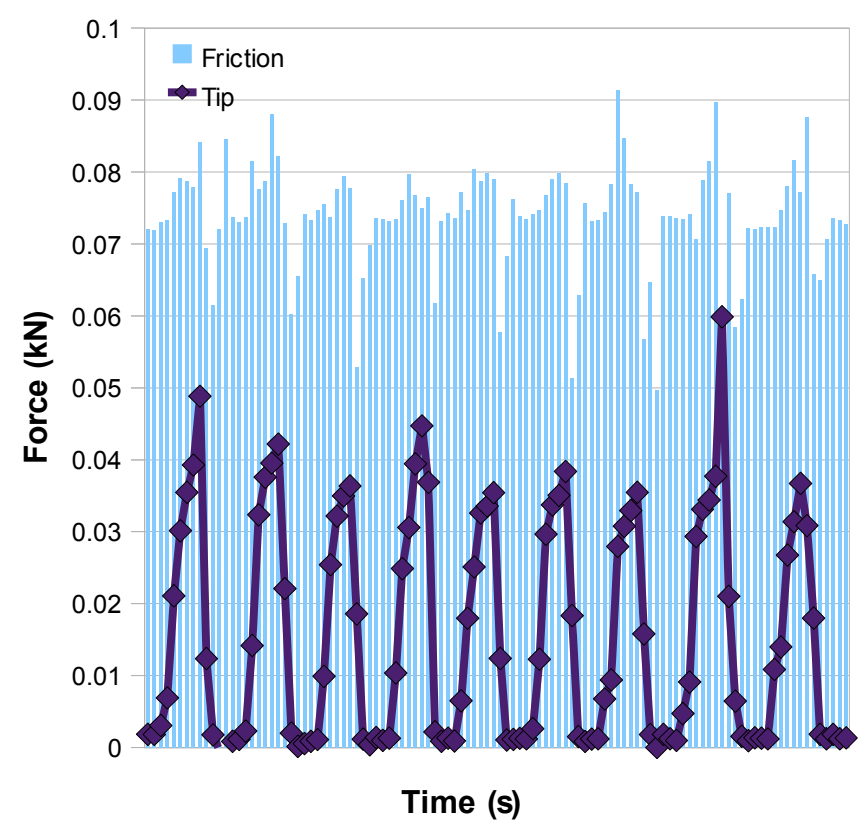

Figure 4.9: Sleeve friction trends with tip resistance during penetration of homogeneous snow. Nine penetration cycles shown. Note that in this figure, with units of force, friction is greater than tip resistance because of the size of the friction sleeve. If units were converted to resistance then tip resistance would be greater than friction.

Similar to tip resistance, sleeve friction also appears greater for higher density snow. Table 4.4 shows mean normalised sleeve friction for Tests 40 to $42(\mathrm{n}=36)$ in homogeneous snow of density $286 \mathrm{~kg} \mathrm{~m}^{-3}$ compared with average sleeve friction for Tests 1 - $9(\mathrm{n}=107)$ in homogeneous snow of density $450 \mathrm{~kg} \mathrm{~m}^{-3}$.

Table 4.4: Variation in mean normalised sleeve friction with density.

$\begin{array}{cll}\text { Tests } & \text { Density }\left(\mathrm{kg} \mathrm{m}^{-3}\right) & \begin{array}{l}\text { Mean Normalised } \\ \text { Sleeve Friction (N) }\end{array} \\ 1-9 & 450 & 73.7 \\ 40-42 & 286 & 71.55\end{array}$

The data suggest that sleeve friction increases with density, consistent with tip resistance. However, statistical testing (unpaired t test with $95 \%$ confidence interval) shows that the difference is not significant $(\mathrm{p}=\sim 0.09)$. This relationship is discussed further with respect to CPT in the next chapter. 


\subsection{Cone effective area}

The fracture of grain bonds and the brittle failure of the snow matrix due to penetration is expected to cause the cone to effect an area larger than its own dimensions: an effective area over which the resistance or force on the cone is expected to be distributed.

To assess the effective area, a series of holes formed by penetration at test site 2 were measured using Silverline analogue vernier callipers, accurate to $0.02 \mathrm{~mm}$. The diameter of the hole formed in penetration was measured at mid depth (to limit hole deformation errors) and then compared with the cone diameter of $11.3 \mathrm{~mm}$. No attempt was made to validate the accuracy of the callipers under test temperatures. Six holes were analysed yielding an average effective diameter of approximately $11.8 \mathrm{~mm}$. Although this value is affected by both measurement inaccuracies and hole deformation during penetrometer removal, this suggests a possible increase in hole diameter of potentially up to $0.5 \mathrm{~mm}$.

Unfortunately the crude nature of this measurement means that this increase in diameter cannot be attributed to either probe movement during insertion and retraction, or a real increase in cone effective diameter because of the breaking and thence carriage of broken snow grains by the penetrometer during the penetration process. However, a real increase in fractured area is expected. This phenomenon is further discussed in Chapter 5 .

\subsection{Formation of compacted zone}

The possible formation of a compacted zone of fractured snow moving ahead of an advancing cone was discussed in Chapter 3. To investigate this phenomenon using the mini-penetrometer, testing was conducted in a layer of homogeneous snow of density $450 \mathrm{~kg} \mathrm{~m}^{-3}$ at site 1 (see Figure 3.2). The snow surface was leveled, and the penetrometer was forced into the snow to a depth of $50 \mathrm{~cm}$. An incision was then made down the centre of the borehole using a hacksaw, and then the dimensions of the penetration cavity were measured and directly compared to the penetrometer.

Unfortunately loose snow grains generated during incision by the hacksaw accumulated in the bottom of the vertical penetration hole, precluding easy visual analysis. In order to remove this obstruction, testing then occurred in a similar manner horizontally, as depicted in Figure 4.10.

Visual comparison between penetrometer and formed cavity was then possible; see Figure 4.11. No evidence was found of the existence of a compacted zone ahead of the cone. This test was repeated numerous times, and on each occasion, no area of compacted snow was visually identifiable ahead of where the cone tip had been. In order to further assess this phenomenon, additional testing occurred whereby the cone was pushed horizontally through a detached block of snow, of density $305 \mathrm{~kg} \mathrm{~m}^{-3}$ 


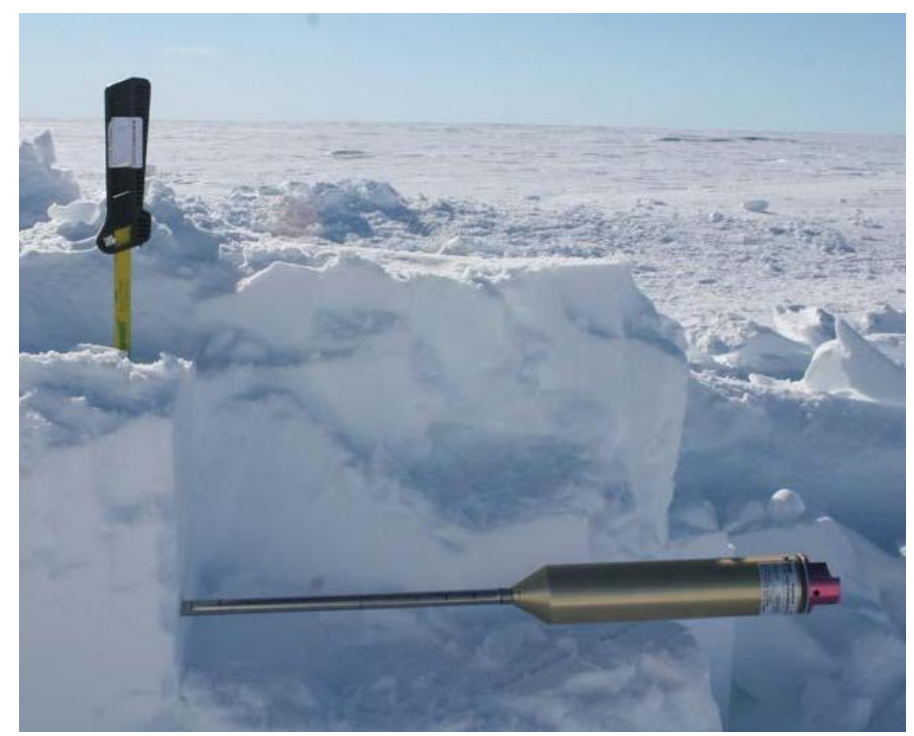

Figure 4.10: Horizontal method of testing for compacted zone ahead of cone.

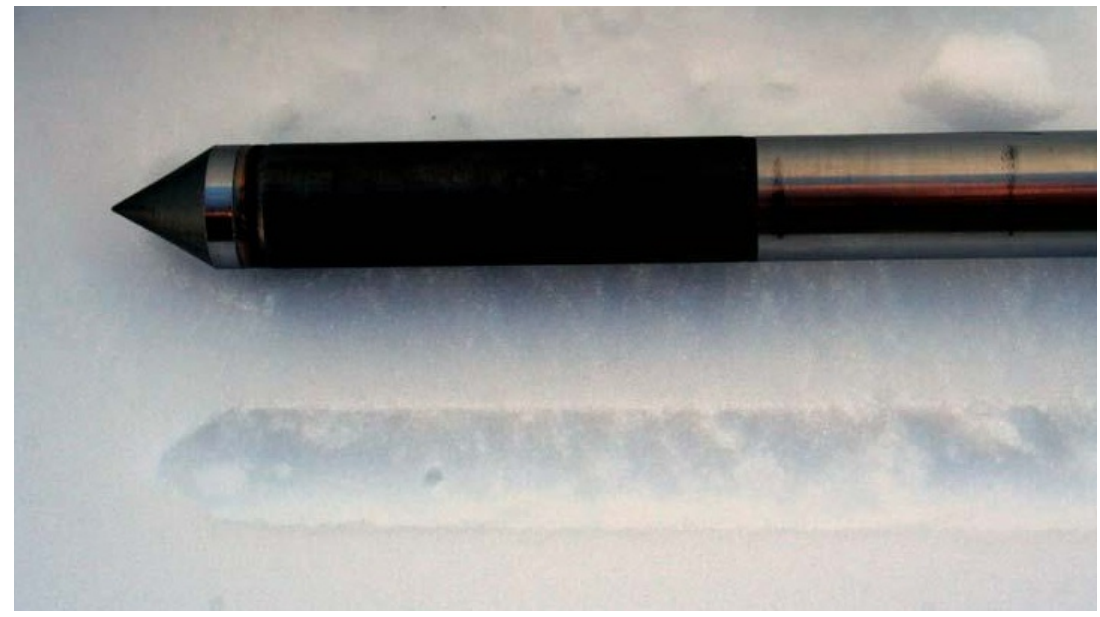

Figure 4.11: Comparison of the penetrometer with the cavity formed from penetration shows no visible evidence of a compacted zone of snow ahead of the penetrometer.

(Figure 4.12).

In this figure a plug of snow is clearly seen to have been forced ahead of the snow as the penetrometer exits the block of snow. This observation appears contrary to the previous observation noting no indication of a compacted zone ahead of the cone. However, it is probably caused by the lack of confinement and the free boundary.

Discussion above concluded that no compacted zone was formed ahead of the cone. This suggests that whilst penetration is occurring (in snow of this or similar density and microstructure) the snow displaced via the penetration process is initially rejected at the surface upon insertion of the penetrometer (such a process was observed and is consistent with observation during shallow penetration testing in snow by Kinosita (1964)), before being preferentially displaced to the side once the cone is fully-engaged within the material. The nature of the snow encountered in Greenland (dry, nonadhesive) and the form and shape of the penetrometer (thin, sharp, $60^{\circ}$ cone angle) 


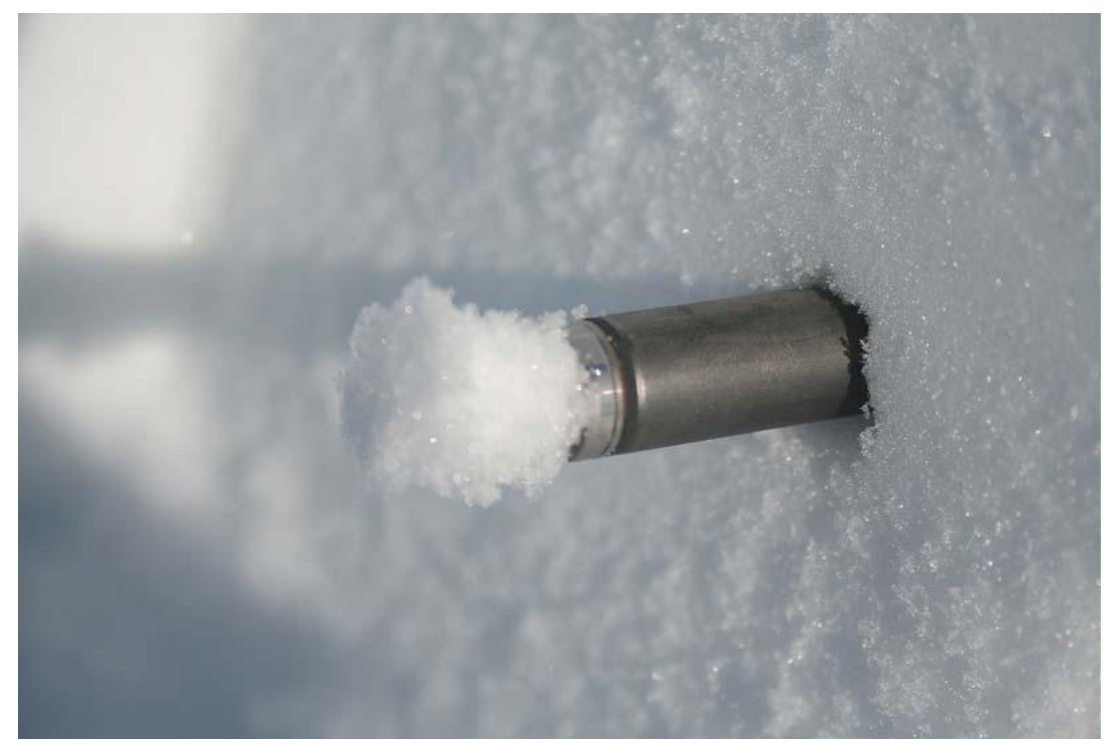

Figure 4.12: A plug of snow was observed to form ahead of the cone upon exit from block of snow.

mean that lateral displacement of fractured material is preferred, rather than the formation of a compacted zone ahead of the cone, such as observed by Kinosita (1964), Floyer and Jamieson (2006) and others.

To investigate this matter a little further, additional testing was carried out using chilli flakes, which have previously been used in assessing the formation or otherwise of a compacted zone ahead of a cone (Floyer and Jamieson, 2006). The aim was to observe whether chilli flakes (of similar dimension to the ice grains) were carried ahead of the penetrometer, or forced to the side during the penetration process. Chilli flakes were either scattered on the snow surface, or scattered within a preformed penetrated hole, and then the penetration test was carried out. Although numerous tests were undertaken, on no occasion were any chilli flakes evident within the snow ejected from the block ahead of the cone. Inspection revealed that the chilli flakes had been displaced to the side of the hole (Figure 4.13).

Testing in Greenland using a sharp, $60^{\circ}$ cone suggests that in dry polar snow, material is preferentially displaced to the side during cone penetration and that a compacted zone does not form ahead of the cone. This phenomenon is discussed further in the next chapter when Antarctic CPT data is examined.

\section{8 'Chipping' of unconfined blocks}

Additional penetrative testing in bonded, medium density $\left(450 \mathrm{~kg} \mathrm{~m}^{-3}\right)$ unconfined blocks of snow at site 4, revealed the brittle fracture or 'chipping' of snow as the penetrometer exited the block (Figure 4.14).

In their work on penetration in porous rock, Leite and Ferland (2001) note that chipping does not occur in very porous materials where compaction occurs via struc- 


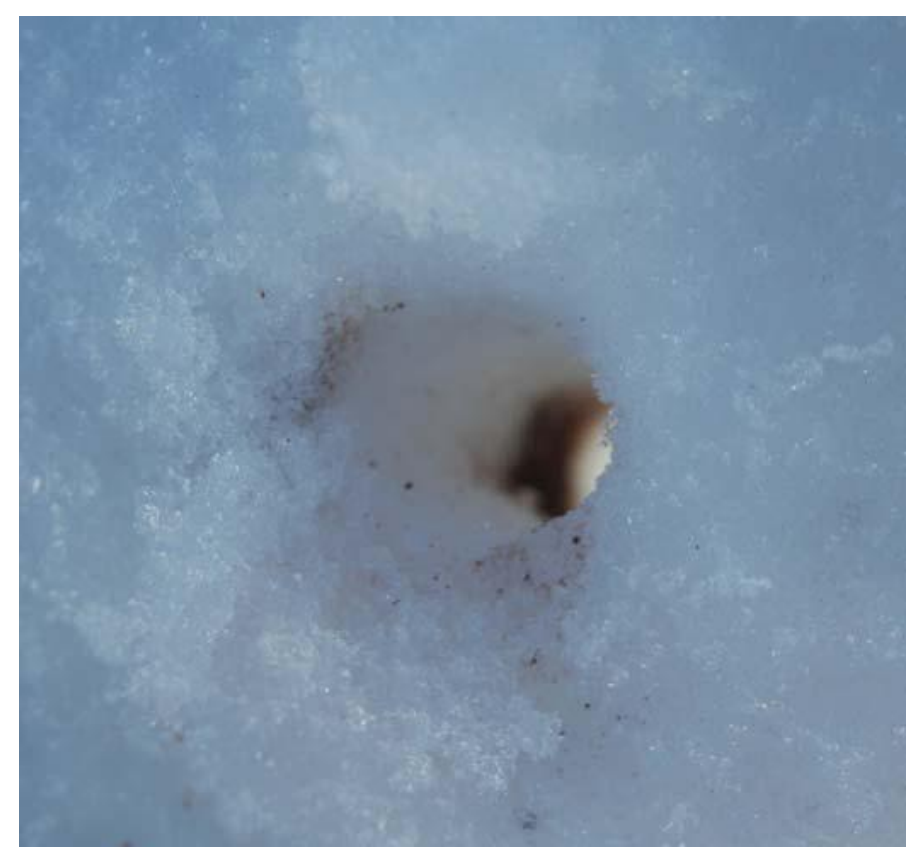

Figure 4.13: Chilli flakes sprinkled onto the snow prior to penetration are shown to have been pressed into the hole sides (dark band) during cone penetration; no flakes were observed in snow ejected ahead of the cone.

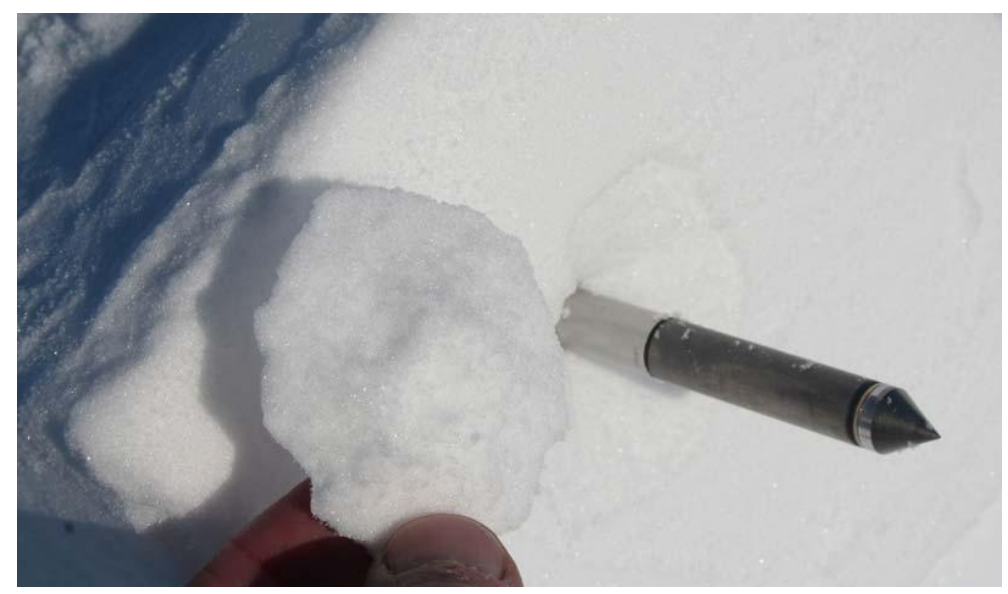

Figure 4.14: During unconfined penetration a chip of snow fractures from the side of a block.

tural collapse. However, it is evident in less porous materials where the opportunity for compaction is limited, and hence the material fractures brittly, as no mechanism to relieve stress internally exists and macro-failure results. Comparison of Figures 4.12 and 4.14 is warranted. Both show penetration through an unconfined surface with one displaying a compacted plug of loose grains, whilst the other shows a fractured chip of material. The difference between the two tests was the density and microstructure of the snow. Snow density and composition appears a major determinant in envisaging the physical processes occurring during both confined and unconfined penetrative processes. Figure 4.15 shows the tip resistance recorded during this process superimposed over load / displacement curves typically observed in rock.

Although both the distance of penetration and the sampling rate is limited, the 


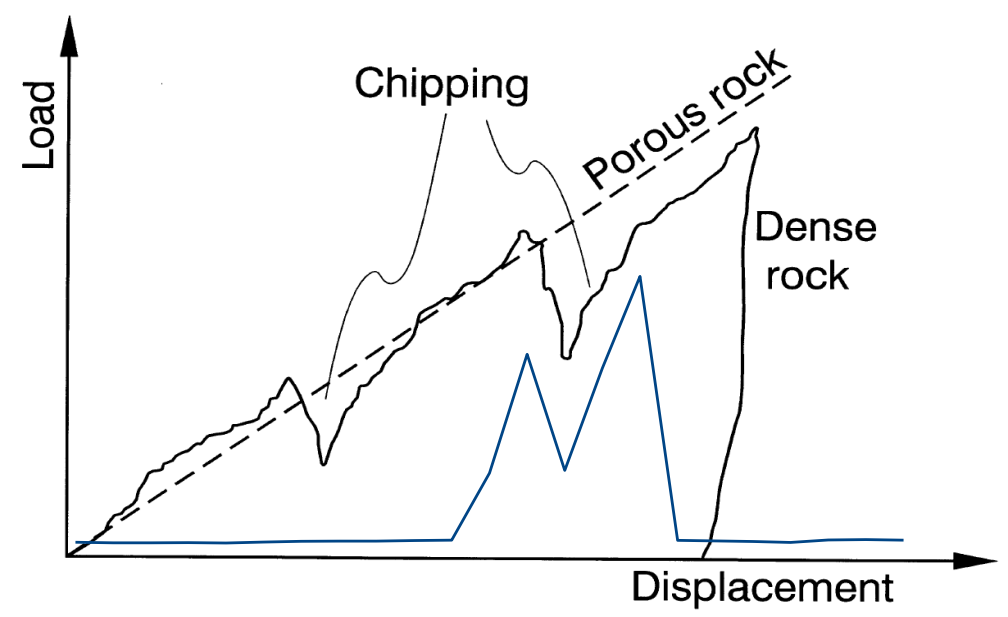

Figure 4.15: Resistance curve during chipping (blue line) superimposed over load / displacement curves typically observed in rock. It is of similar form to the chipping curve seen for dense rock, suggesting low porosity.

tip resistance curve is similar in form to the 'chipping' curve for rock (Leite and Ferland, 2001), also displayed in Figure 4.15, typical in rock of reduced porosity. Clearly, as described in Chapter 2, the behaviour of snow is not dissimilar to that of other geomaterials.

\subsection{Summary}

Preliminary penetrative testing was undertaken in Greenland using the Fugro minipenetrometer in order to provide additional data for comparison with the larger-scale hydraulic CPT performed in Antarctica. This chapter provided some understanding of the main questions posed in Chapter 1 along with some of the specific questions that were outlined in Chapter 3. Interpretation discussed here will be considered further with respect to findings from the Antarctic CPT in the next chapter. 


\section{Chapter 5}

\section{Results \& Analysis (B) - Cone Penetration Testing}

The previous chapter discussed data obtained during a pilot study conducted in Greenland using the Fugro mini-penetrometer. In this chapter, results are presented and discussion ensues on the major component of this research, CPT in Antarctica. This research occurred in the vicinity of Halley V Research Station, over the period January and February 2010; a table summarising the ninety-three tests conducted is at Appendix A. Because of the many sections in this chapter, each section is largely 'selfcontained' for clarity; included are both the results, and analysis and discussion of each facet under examination. Specific questions raised in Chapter 3 are also addressed.

\subsection{CPT results}

The majority of CPT was conducted to a depth of $\sim 5 \mathrm{~m}$, or until excess vibration caused early test termination. Penetration rates were varied, and cone tip resistance $(\mathrm{MPa})$ and sleeve friction $(\mathrm{MPa})$ were recorded for each test. Except for a number of anomalous situations as described later, no pore pressure readings $(\mathrm{kPa})$ were obtained. CPT was conducted in many different locations (see Figure 3.5) and not all data are shown here. Figure 5.1 is a screen capture from the Golog software showing unprocessed data from a typical cone penetration test, Test 37 (to $10 \mathrm{~m}$ ). Tip resistance (MPa) is shown on the left and sleeve friction $(\mathrm{MPa})$ on the right. Pore pressure $(\mathrm{kPa})$ was recorded but is not shown in this figure.

All CPT data for a standard cone, obtained within $1 \mathrm{~m}$ of test pit \#1 are shown in Figure 5.2 and all CPT data obtained within the vicinity of test pit \#2 are shown in Figure 5.3. Tests were conducted at different rates and the average data for each location is shown as a bold black line.

Initial observation of these figures reveals a drop in resistance every $\sim 0.5 \mathrm{~m}$ where rods are changed, a high degree of consistency between tests and immediate evidence of harder strata. CPT was conducted in order to investigate specific phenomena and 


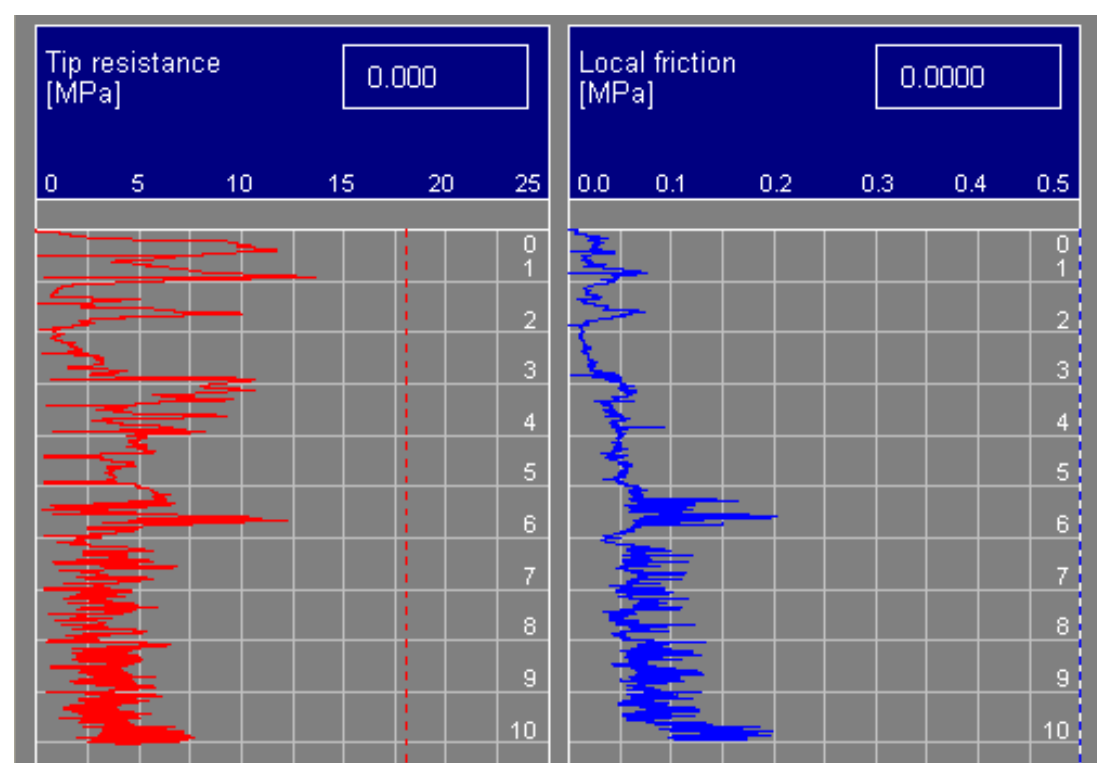

Figure 5.1: Screen shot showing typical CPT data. Tip resistance is on the left in red and sleeve friction is on the right in blue. Test was to a depth of $\sim 10 \mathrm{~m}$.

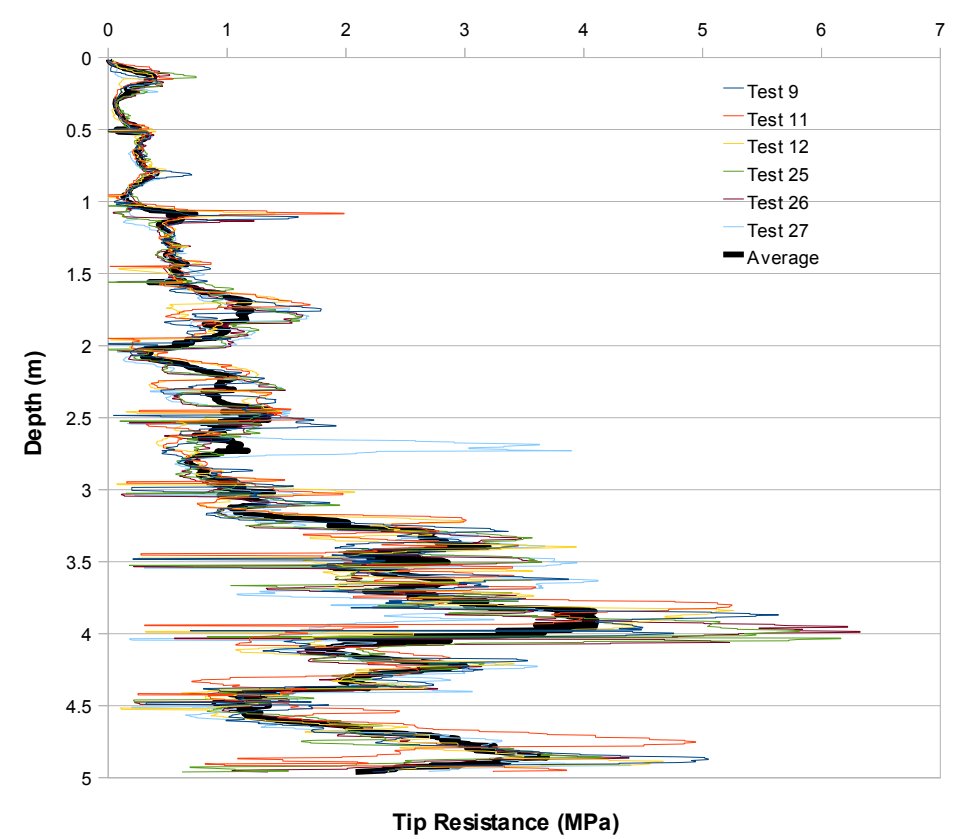

Figure 5.2: All CPT data from tests conducted adjacent to test pit \#1. The bold black line is the mean resistance value generated from all tests.

additional testing data is now presented as necessary to allow analysis and discussion.

\subsection{Basic Signal Analysis}

A level of basic signal analysis was conducted on both the tip resistance and sleeve friction trace in order to gain some understanding of the nature of these signals. Further discussion on each signal occurs in later sections. 


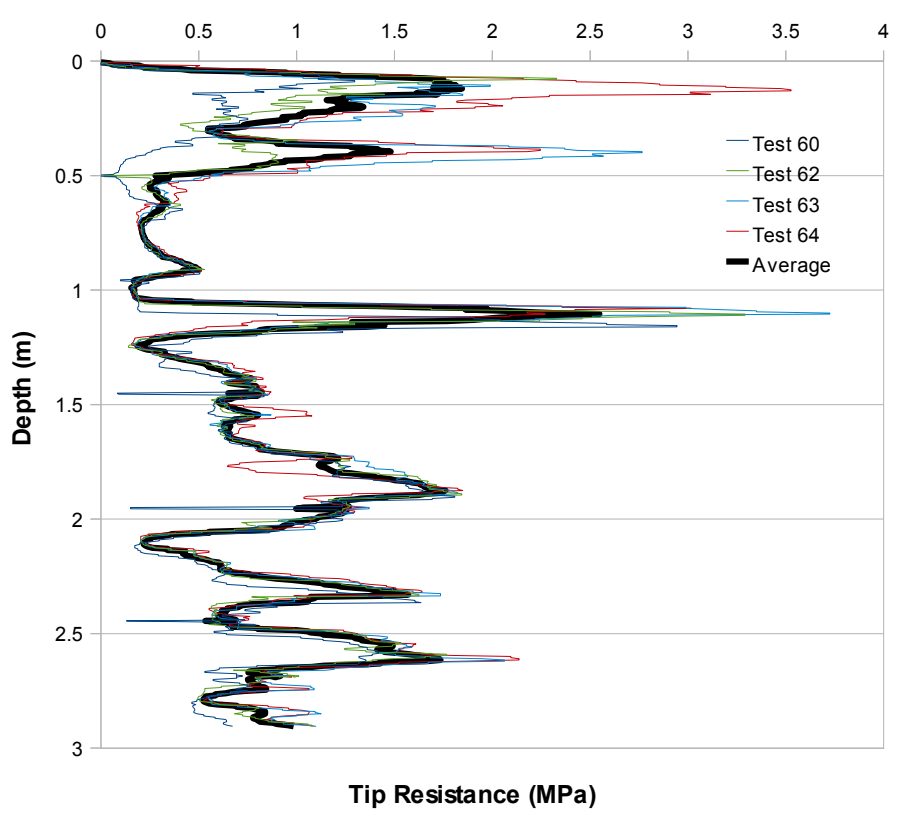

Figure 5.3: All CPT data from tests conducted adjacent to Test Pit \#2. The bold black line is the mean resistance value generated from all tests.

\subsubsection{Tip Resistance}

Rapid penetration of a small object in snow is usually characterised by a rising 'sawtooth' trace as fracture of bonds on the microscale is superimposed over the resistance increase owing to macroscale compaction and densification. Such behaviour was observed by Kinosita (1967) and is described by numerous authors including Mellor (1975): "The motion may involve a series of discontinuous collapses". The limited data-collection rate available during CPT precluded the identification of $\mathrm{mm}$ - scale (grain/bond scale) saw-tooth patterns. However, an irregular trace suggesting similar behaviour can be observed in many of the tip-resistance traces presented thus far. It is of interest to compare CPT tip resistance traces (such as in Figures 5.1, 5.2 and 5.3) with a sample SMPT trace. The SMPT collects data every $0.5 \mathrm{~mm}$ and exhibits an obvious saw-tooth pattern displaying fracture at a microscopic level; see Figure 5.4 which is a schematic of SMPT tip resistance in poorly bonded snow (top) then well-bonded snow (bottom).

Force-distance data due to the breaking of individual grain bonds can be captured by the SMPT whereas at a sampling dimension of $5 \mathrm{~mm}$, such behaviour will not be evident in the CPT tip resistance signal. Simple inspection of a Fast-Fourier Transform (FFT) of a typical CPT trace (Figure 5.5) shows that most of the variation in the CPT tip-resistance signal is caused by low frequency events such as different strata as opposed to high-frequency variations, say owing to snow microstructure.

The limited resolution of the CPT equipment used suggests that the saw-tooth patterns are probably not owing to the fracturing of ice bonds/grains at a micro level, but may actually be induced by slip-stick behaviour of the cone friction sleeve also 


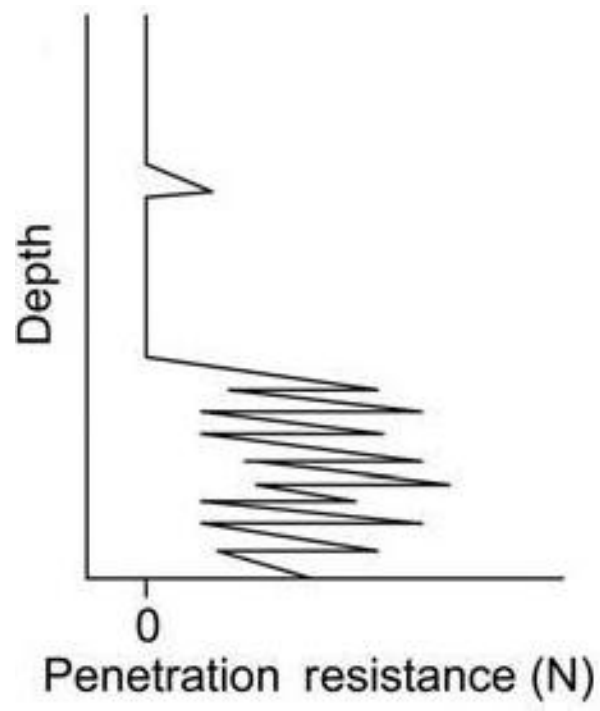

Figure 5.4: Schematic of SMPT tip resistance in poorly bonded (top) then well-bonded snow (bottom) shows 'saw-tooth' behaviour in well-bonded snow (taken from Bellaire et al. (2009)).

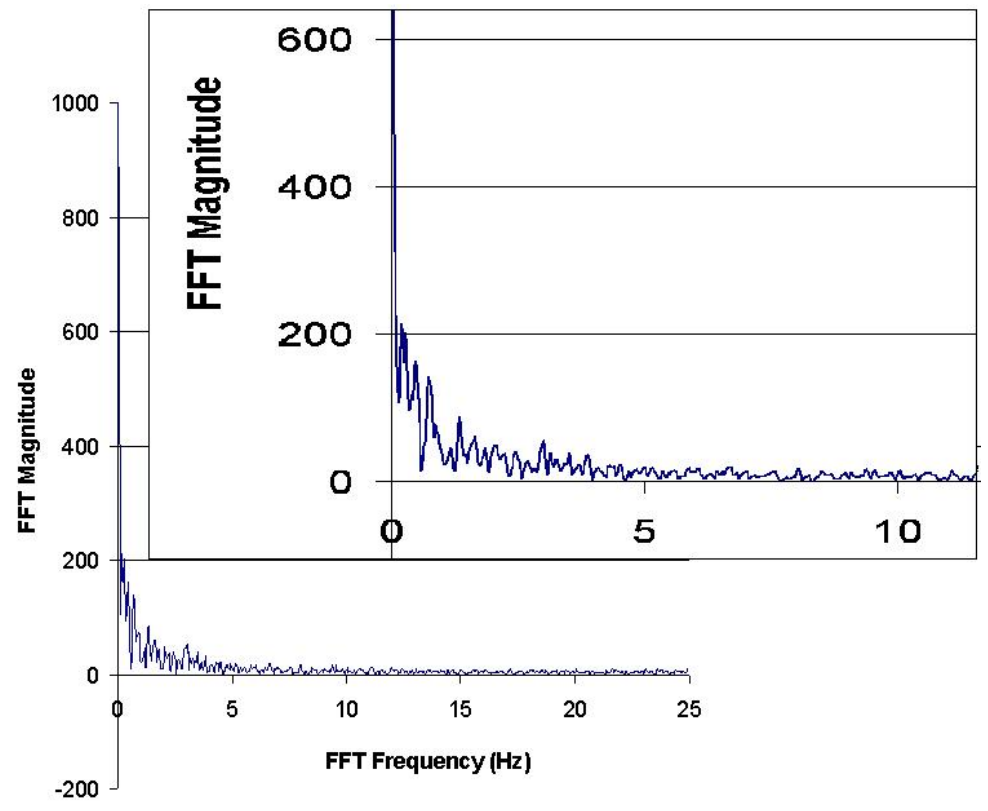

Figure 5.5: Fast Fourier Transform of tip resistance (Test 9) shows that most variation within the tip-resistance signal is owing to low frequency events.

affecting the tip resistance trace. The nature of the sleeve friction trace is discussed next.

\subsubsection{Sleeve Friction}

The friction data retrieved via CPT are averages measured over the $135 \mathrm{~mm}$ length of the friction sleeve and will also lag the tip resistance trace because of the physical location of the friction sleeve, some $90 \mathrm{~mm}$ behind the cone tip.

Close inspection of a CPT friction-sleeve trace (Figure 5.6) sometimes reveals erratic 
'saw-tooth' like behaviour. However, in this case such behaviour is not owing to fracture of bonds at a microstructural level (and is not necessarily superimposed upon a rising signal), but is more likely because of frictional 'stick-slip' behaviour.

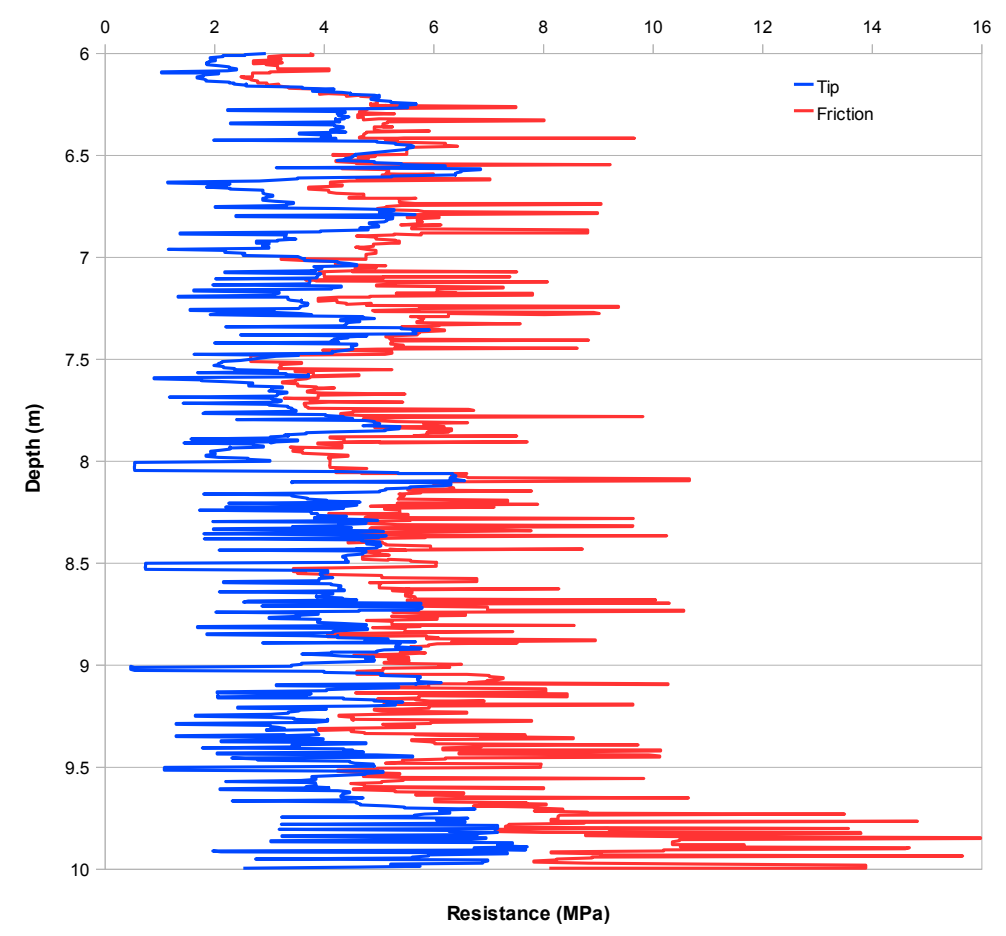

Figure 5.6: The frictional trace (red) from Test 10 shows typical 'stick-slip' behaviour from 6 to $10 \mathrm{~m}$ depth that is also apparent within the tip resistance trace (blue). Such behaviour could be minimised by using a 'friction-reducer' mounted on the rod directly behind the cone.

Such behaviour is apparent across the scales in ice and is also observed in alpine and polar glaciers (Bartholomew et al., 2010). Figure 5.6 also shows that slip-stick behaviour induced due to friction between the cone and rods and the snow is also evident in the tip-resistance trace, thus potentially generating unreliable or at least noisy tip resistance data when significant stick-slip behaviour occurs.

\subsubsection{Friction Ratio}

The friction ratio $\left(R_{f}\right)$ is equal to sleeve friction divided by tip resistance $\left(f_{s} / q_{c}\right)$ x 100 $\%$. In standard cone penetration testing analysis and interpretation, the friction ratio is of primary importance. It is the ratio that generally differentiates between sandy and clay soils, and is often the primary determinant in classifying a soil. In snow, a vast range of material types does not exist, and because tip resistance and sleeve friction particularly are not previously known to have been recorded or analysed in snow, then appropriate use of this parameter is still open to debate. The friction ratio signal exhibits behaviour consistent with both the tip resistance and friction sleeve trace, and to obtain a true friction ratio, the lag between tip and sleeve must be taken into account. This 'shift distance' is typically measured by subjective or empirical means. 
However, Jaksa et al. (2002) suggest that the cross correlation function (CCF) can be used between tip resistance and sleeve friction data to derive a more representative 'shift distance' between the tip and sleeve. The CCF between tip resistance and sleeve friction from a representative CPT Test is shown in Figure 5.7.

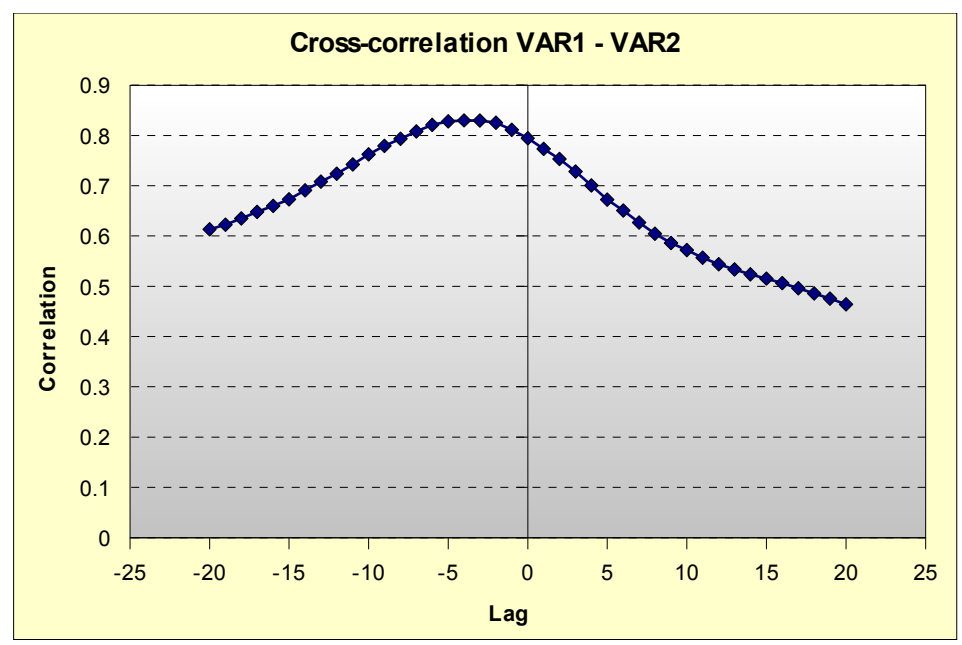

Figure 5.7: Positive correlation exists between sleeve friction and tip resistance. Lag due to the physical dislocation of the sensors is apparent.

In this figure the lag between the two readings is shown to be approximately six units of measurement, or $30 \mathrm{~mm}$, owing to the $5 \mathrm{~mm}$ sampling rate. The determination of this 'shift distance' has direct implications on the calculated friction ratio at all depths, thus determination via the most accurate means is desirable. This topic has only been briefly examined above and Lunne et al. (1997) describe other statistical approaches and techniques which may be employed in the interrogation of CPT data. Further discussion on the use of the friction ratio occurs in the next chapter.

\subsubsection{Pore Pressure}

Surprisingly, although no free water existed in the dry snow tested, pore pressure readings were observed during a number of tests adjacent to snow pit \#2 and within the vehicle park; an example is presented in Figure 5.8 (pore pressure is normalised).

It was initially considered that this pore pressure reading was some measure of the true variation in (air) pore pressure within the snow, so the cone used for these tests was inserted into the holding-tank of an air-compressor to verify its responsiveness to changes in air pressure. It did respond quickly and accurately to generated air pressure changes hence the possibility of true air pore-pressure readings being observed remained. However, the often exact alignment of the pore pressure trace with the tip resistance trace aroused suspicion. Lunne et al. (1997) note that on occasion, equipment-related pore pressures may be generated on the cone because of high compressive stresses. The anomalous pore pressure readings noted above were observed in 


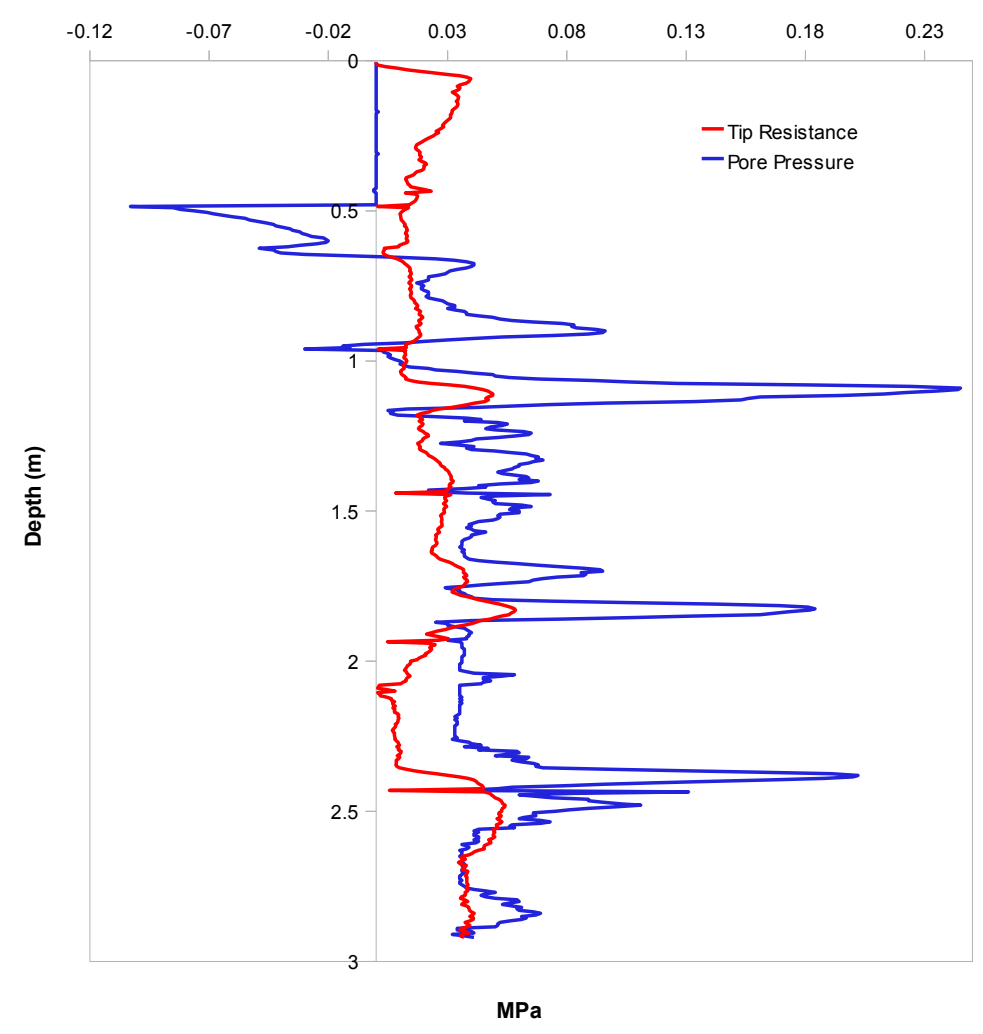

Figure 5.8: Anomalous pore pressure reading observed post sea-ice testing is assumed to be due to 'cross-talk' between the cone tip and the pore pressure transducer.

tests conducted after testing in sea ice in the vicinity of Halley V. This testing is not discussed within this thesis but during these tests, tip resistance exceeded $40 \mathrm{MPa}$, beyond the rated range of the cone.

A schematic of the cone's pore pressure sensing system (Figure 5.9) shows how this 'cross-talk' might occur. With the pressure load cell located immediately behind the cone tip, any solid ice frozen within the pore-pressure channels may assist in the direct mechanical transfer of load between the cone tip and the pore pressure transducer.

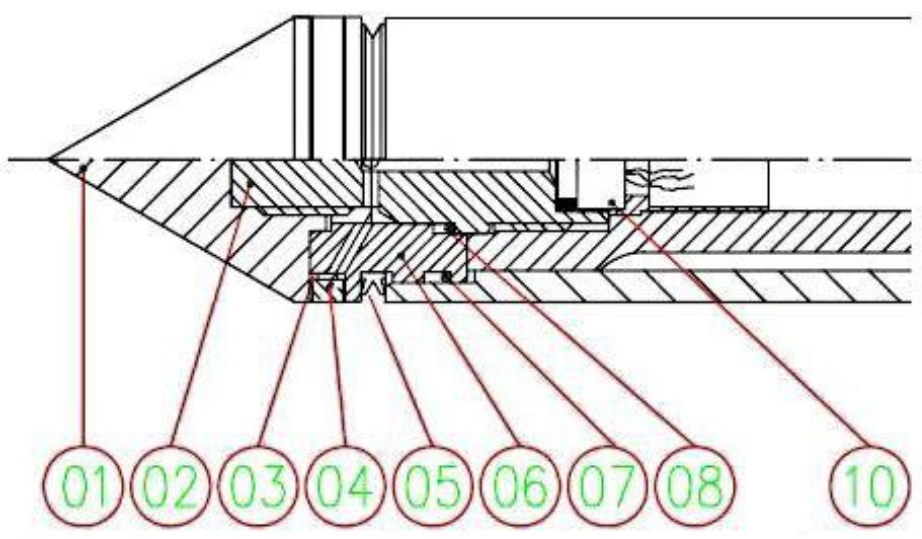

Figure 5.9: Schematic of cone pore-pressure measuring system (courtesy GeoPoint Systems BV).

The sea ice tests were the first tests conducted in the presence of free-water and it 
is assumed that frozen sea water remained within the pore-pressure channels and thus when the tip was next loaded, mechanical loading of the pore pressure sensor occurred, resulting in an erroneous pore pressure signal.

The realisation that this pore-pressure signal was mechanically generated was disappointing, as the ability to obtain pore pressure data from within the snow would be very interesting. Using current equipment, the gathering of such data does not appear possible. To clarify: detection of liquid pore pressure remains possible but the gathering of air pore pressure may only be possible by using appropriately calibrated and load-cell equipped penetrometers.

\subsection{Variation of signals with depth}

\subsubsection{Tip Resistance}

A cone penetrometer tip resistance reading in soil is very heavily influenced by the vertical effective stress within the soil, with measured cone tip resistance $\left(q_{c}\right)$ typically corrected for in situ effective vertical stress to provide a net cone resistance $\left(q_{n}\right)$. A similar modification is expected to be necessary when interpreting cone tip resistances measured in snow. However, it is surmised that vertical stress has less effect on readings within snow because the unit weight of snow is typically an order of magnitude less than that of soil. Soil is largely unbonded hence an increase in normal force (overburden) may result in greater frictional strength of the soil. This is not expected in (typically) bonded snow where frictional forces are not yet mobilised.

The estimated vertical effective stress profile within the snow adjacent to Snow Pit \#1 is shown in Figure 5.10. The in situ stresses $(\mathrm{kPa})$ are an order of magnitude less than typical tip resistances measured during penetration ( $\mathrm{MPa}$ ), so although consideration of vertical effective stress is necessary, limited impact upon measured tip resistance values is expected.

The extent that in situ stresses may have on measured tip resistance is further considered in section 5.15, Effect of confining pressure.

\subsubsection{Sleeve Friction}

It was surmised above that normal stress due to overburden pressure may have less effect on measured tip resistance in snow because of the bonding that typically exists. However, sleeve friction is obtained from previously fractured, now individual, grains of snow and an increase in normal stress may be expected to have an effect. If so, measured sleeve friction should increase with depth as overburden increases. However, demonstrating this effect is not straight-forward, because of the complicated interaction of snow density, microstructure, stratigraphy etc. that may influence measured sleeve 


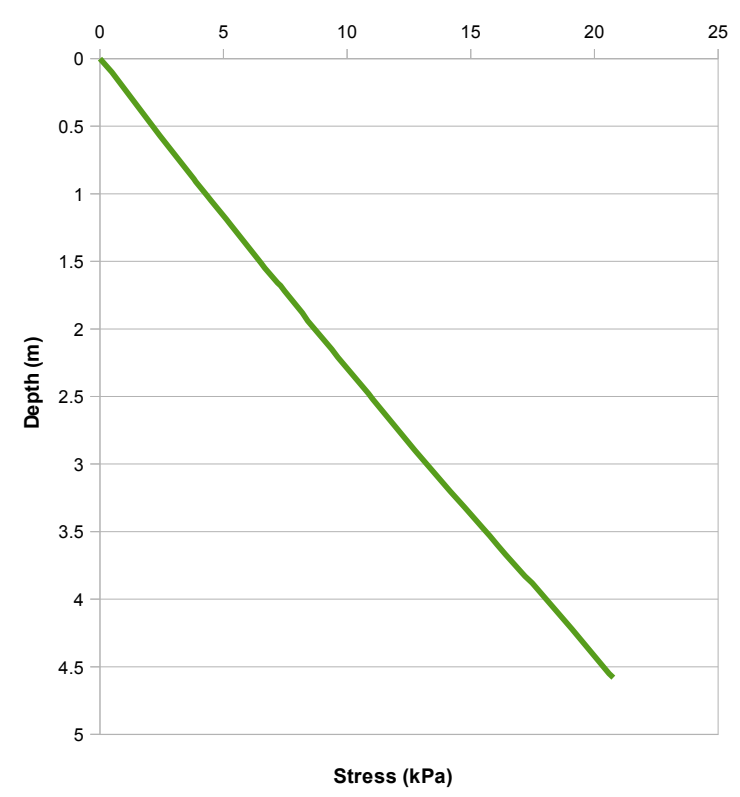

Figure 5.10: Estimated in situ vertical stress at snow pit \#1. In situ stresses $(\mathrm{kPa})$ are one or two orders of magnitude less than typical tip resistances (MPa).

friction values. Therefore, evidence for this effect is discussed at a later stage in the section on overburden pressure.

\subsection{Variation of tip resistance with sleeve friction}

Variation of tip resistance with sleeve friction is evident throughout the testing. In some tests, friction was still rising within a layer, whilst tip resistance had already reached a peak and was decreasing. As previously discussed, the distance of the lag between these parameters does not necessarily correspond to their physical separation, but is at a distance preferably determined through the use of a comparative cross correlation function or similar method. The lag determined via this method is seen to vary with the speed at which a test was conducted, and may also vary owing to the nature of the snowpack: stratigraphy, density, average resistance etc., hence ideally a CCF or similar should be performed upon each test so that the lag and thus the correct friction ratio can be calculated. The variation of tip resistance and sleeve friction with density is discussed in a subsequent section.

\subsection{Spatial Variability}

In addition to tests conducted in close proximity as previously described, further tests were conducted to specifically examine spatial variability of resistance within the snow pack. Tests 25 to 31 were conducted at increasing increments $(0.1 \mathrm{~m}, 1.0 \mathrm{~m}, 10 \mathrm{~m}$, $100 \mathrm{~m}$ ) out to a distance of $1 \mathrm{~km}$ to investigate spatial variability over a larger scale. 
Spatial layer continuity from these tests is shown in Figure 5.11, a two-dimensional spatial plot of cone tip resistance. All tests were conducted with a standard cone at a rate of $24-25 \mathrm{~mm} \mathrm{~s}^{-1}$, to a depth of $5 \mathrm{~m}$; note the logarithmic distance scale $(\mathrm{m})$; colour table represents tip resistance in MPa.

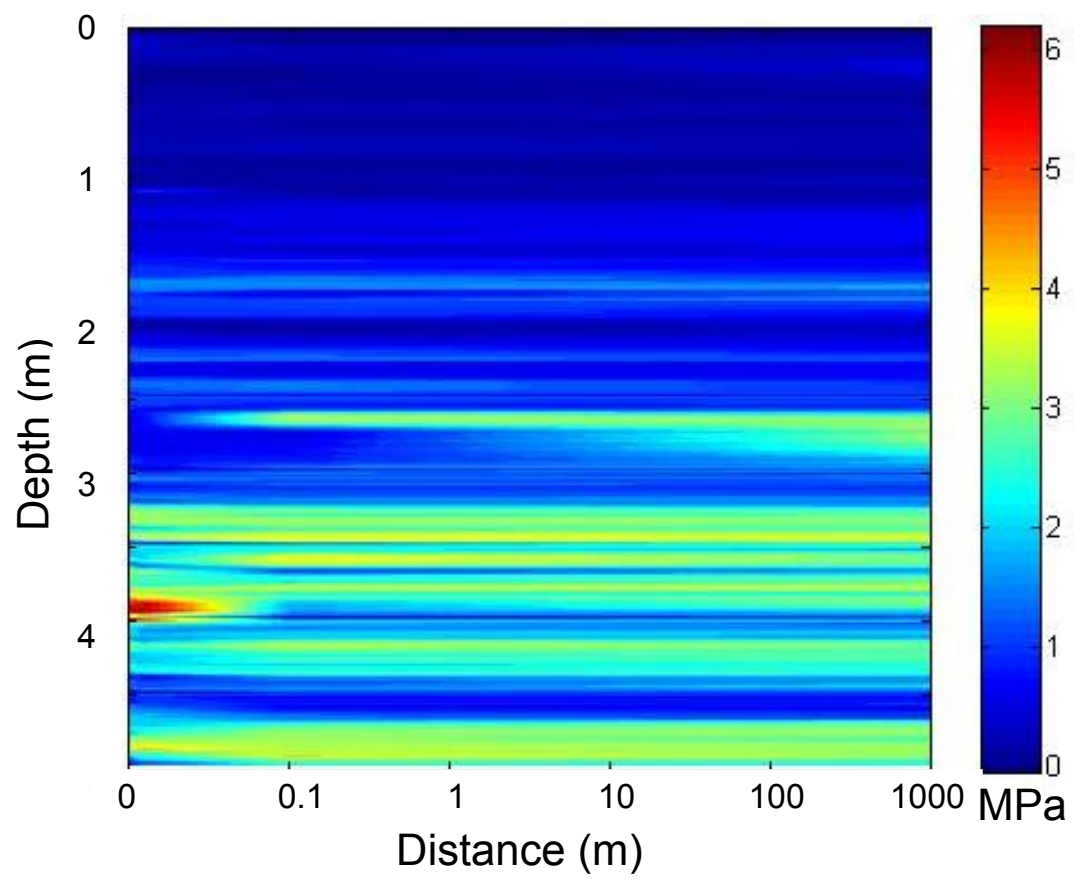

Figure 5.11: Two-dimensional spatial plot of cone tip resistance shows spatial continuity of resistance horizons; colour table represents tip resistance in MPa.

Tests $9,10 \& 12$ were conducted within $\sim 2 \mathrm{~m}$ of each other. Basic statistical data comparing these tests suggests that quantitatively, limited small scale variability exists between tests (Table 5.1). Frictional data from these tests displays similar limited variability both qualitatively and quantitatively.

Table 5.1: Basic Statistics - Tests 9, $10 \& 12$

\begin{tabular}{|c|c|c|c|c|}
\hline Test & $\begin{array}{l}\text { Mean } \\
(\mathrm{MPa})\end{array}$ & $\begin{array}{l}\text { Standard } \\
(\mathrm{MPa})\end{array}$ & Deviation & $\begin{array}{l}\text { Bivariate Correlation } \\
\text { (with Test 9) }\end{array}$ \\
\hline 9 & 1.36 & 1.43 & & 1.0 \\
\hline 10 & 1.31 & 1.15 & & $0.81^{1}$ \\
\hline 12 & 1.32 & 1.16 & & $0.89^{1}$ \\
\hline
\end{tabular}

Figure 5.11 suggests that some degree of horizon consistency is evident over a distance of $1000 \mathrm{~m}$ and is consistent with the GPR trace over the same transect (Figure 5.12). Further comparison between CPT tip resistance and GPR occurs in the next chapter.

Sturm and Benson (2004) conclude that at a scale of $10 \mathrm{~m}$ or less the spatial heterogeneity of perennial (polar) snow is limited. Such uniformity may be expected in an area such as the Halley site where sources of snow heterogeneity such as topography, 


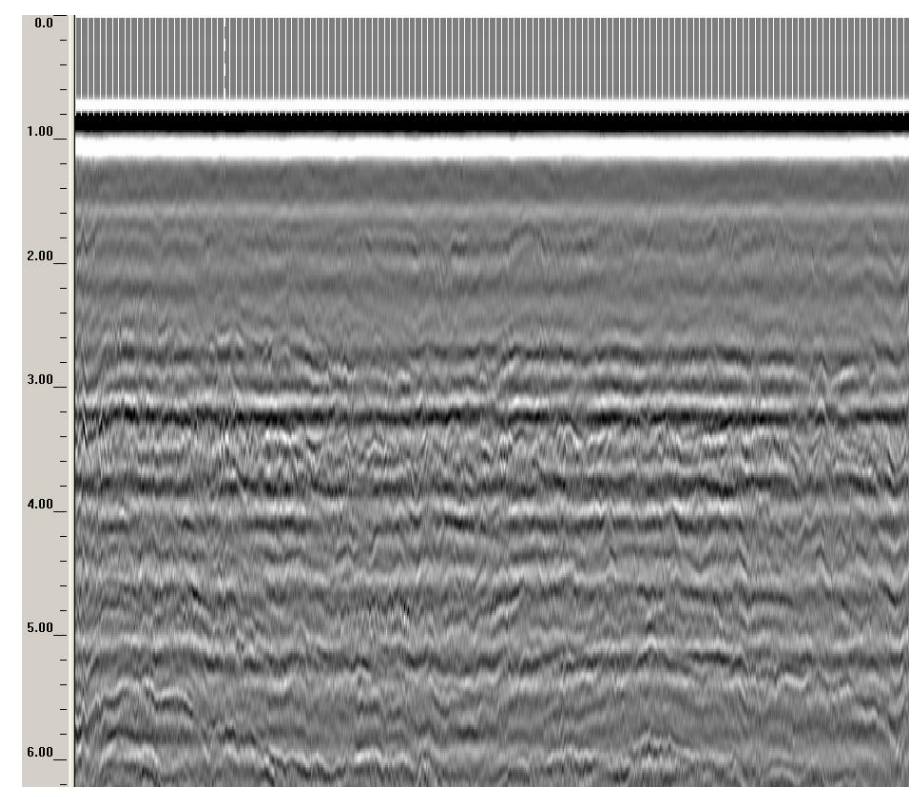

Figure 5.12: GPR linescan to $6.2 \mathrm{~m}$ depth along line of spatial CPT testing (0 - 1000 $\mathrm{m})$ showing consistency of layers. Data is horizontally stacked 20-fold.

vegetation and wind either are non-existent or of limited variation. Such terrain typical of Halley is shown in Figure 5.13.

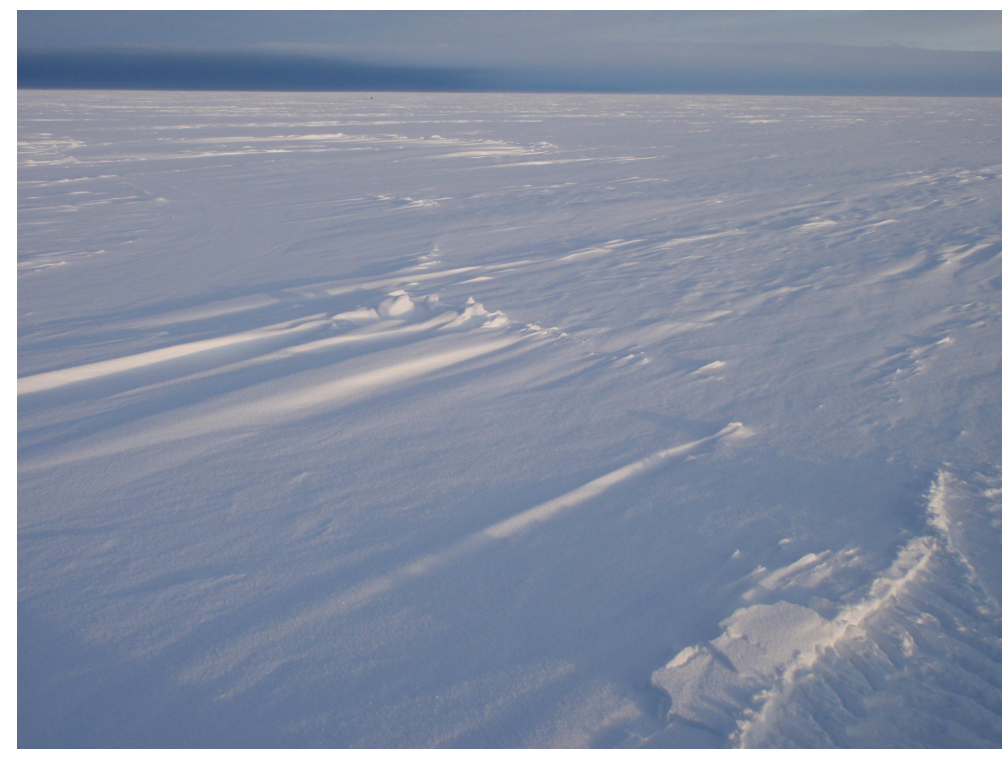

Figure 5.13: Snow surface, vicinity Halley V Research Station showing limited heterogeneity (tractor tracks bottom right of picture for scale).

Schweizer et al. (2008a) carried out testing into variation of snow surface properties across various scales in the alpine environment and found that in areas of limited topographical variation, experiencing similar wind regimes (such as at Halley), variation in surface snow properties was limited at the basin scale $(\sim 1000 \mathrm{~m})$. Chiaia and Frigo (2009) in developing a model for snow slab avalanches also observed the scale invariance of snow, citing work by Faillettaz et al. (2003) on the scale invariance in the behaviour of almost 5000 avalanches in Europe, and Haegeli and McClung (2003) 
report the existence of consistent weak layers over scales of hundreds of kilometres in alpine snowpacks. Such extensive limited heterogeneity was also shown in Antarctica by Arcone et al. (2004) who tracked consistent horizons via GPR for hundreds of kilometres. Sturm et al. (2004) in a study in sub-Arctic Alaska conclude that large scale variability is limited, however, variation on the smaller scale is greater, largely because of wind.

When findings suggesting limited large scale variability are considered in conjunction with evidence by Sturm and Benson (2004) suggesting limited variation on the small scale also, then large scale invariance of snow properties may be expected at a site such as Halley. Such evidence may mean that optimisation of site characterisation by CPT using an uncertainty based method (such as proposed by Ng and Zhou (2010)) may be possible. However, a database does not yet exist to readily utilise such a method in snow. With increased work, similar methods to optimise CPT utilisation in characterising a snow site may prove of value.

This discussion highlights that consistency in resistance measurements at Halley is apparent across limited spatial scales, that limited heterogeneity is expected at the Halley site, and that if CPT resistance can be correlated with GPR amplitudes (as discussed in Chapter 6) then resistances should be extrapolatable spatially through the use of GPR, potentially precluding the conduct of unnecessary point cone penetration tests when characterising a site.

\subsection{Qualitative spatial comparison of CPT data}

Spatial consistency of the CPT data has been discussed above. This section briefly highlights the extremely valuable qualitative information that can be obtained from CPT for comparison of layering and resistance within the snowpack, before quantitative analysis is discussed. Figure 5.14 shows variation in tip resistance at numerous sites within the vicinity of the Halley V Station; average data from test pit \#1 is shown again for comparison.

Without understanding any of the physical processes or having a quantitative appreciation of resistances generated, a figure such as this can be of enormous benefit in assessing changes in snow pack structure both temporally and spatially.

Areas where substantial snow processing and compaction has occurred over many years such as the Vehicle Park and Relief Road display substantially greater resistance than less-worked or virgin snow areas, providing immediate guidance to engineers on relative strengths. Comparative testing of these areas can occur throughout construction or on a seasonal basis to further document qualitative increases in observed snowpack resistance. The use of the CPT as a rapid, qualitative snow resistance assessment tool, to depth, in polar areas is undeniably validated. 


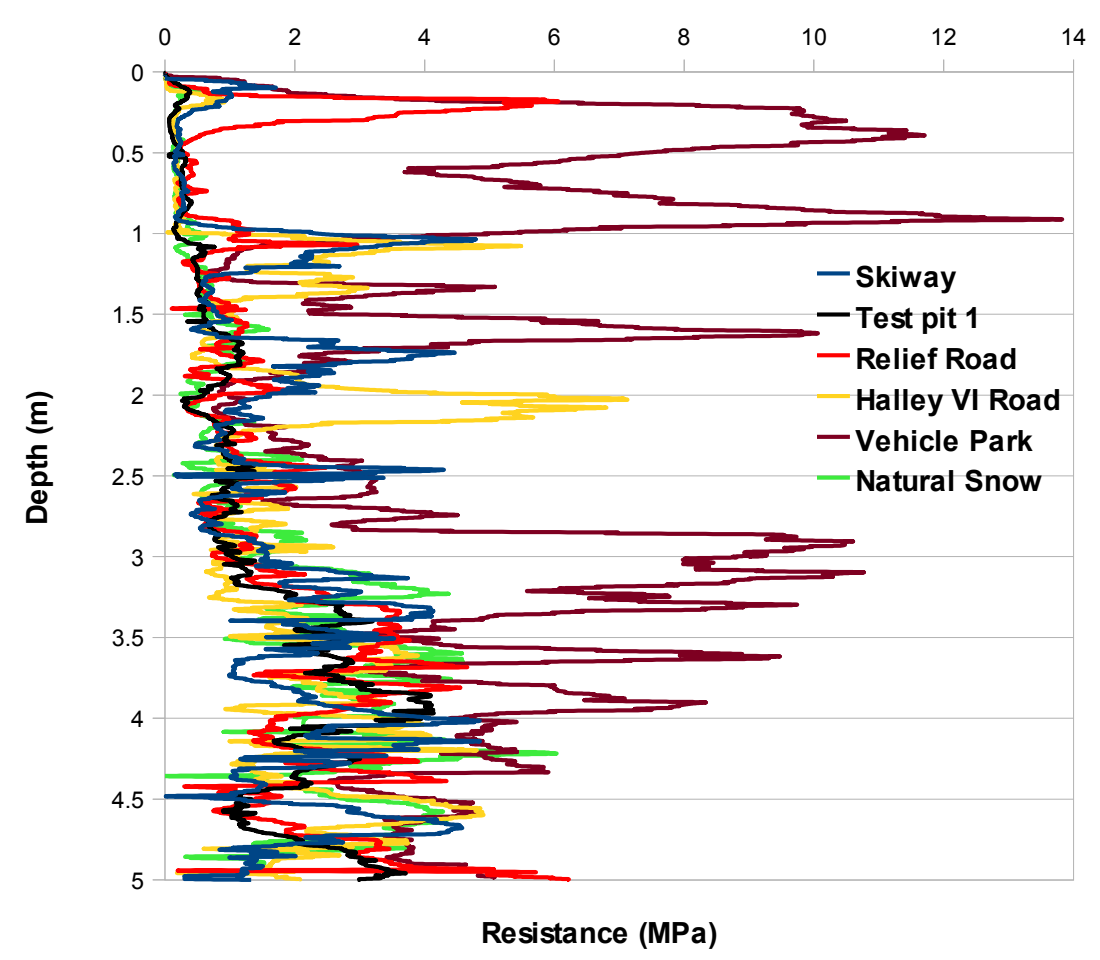

Figure 5.14: Qualitative resistance comparison from numerous Halley V sites shows the strength increase obtained by compacting snow, particularly obvious from the Vehicle Park data.

\subsection{Effect of grain size}

It is very hard to separate the effect of grain size on cone penetration results from that of density as the two were found to be highly-correlated. However, possible variation in both tip resistance and sleeve friction owing to grain size is discussed below.

Creep-rate dependence on grain size has been investigated by numerous authors (Kubo et al. (2006), Sammonds (2006), Schulson (2001), etc.). However, less work is evident on the effect of grain size on penetrometer testing. Floyer and Jamieson (2006) suggested that grain size affects the formation of the compacted zone ahead of a penetrometer in snow and Schweizer (1999) reported that increased grain size tends to shift the brittle-ductile transition towards lower strain rates. Thus, at the same penetration rate (not necessarily the same local strain rate at the penetrometer tip) decreased resistance values may be expected in the brittle zone if larger grain sizes are encountered. As grain size generally increases with depth, some level of decreased tip resistance may therefore be expected with depth. So changes in grain size may have some limited effect on measured tip resistance.

Limited literature is available to guide discussion on sleeve friction measurements in snow because no such testing is known to have previously occurred. However, from a planar friction perspective, Bowden (1953) considering grain size in snow as a measure of surface roughness, suggests that smaller grain size may enable greater packing, resulting in an appreciable increase in surface area. This would mean that 
sleeve friction measurements may be greater in snow of smaller grain size, all other parameters being equal. Ericksson (1955) also showed that friction increases with smaller grain size. Thus sleeve friction may be expected to decrease by some amount with depth as average grain size increases.

Ideally, some measure of these variations would be extractable from the CPT results. However, because of the complicated stratigraphy and other material changes evident within each test, isolation of this phenomenon is not readily achievable. Often lower density snow has a larger grain size and thus the effect on resistance because of density may tend to override (in the opposite sense) any variation owing to grain size. Controlled laboratory tests in homogeneous blocks of snow where both grain size and density (as well as microstructure) can be manipulated would appear necessary to accurately resolve this relation with regards to penetrative testing in snow. The effect of grain size on the cone effective area is discussed shortly.

\subsection{Effect of penetration rate}

Ninety-three cone penetration tests were conducted at various rates, allowing rate effect of penetration to be considered; tests 15 to 20 were specifically conducted to assess rate effects on both measured tip resistance and sleeve friction. Penetration rates varied from 0.16 to $55 \mathrm{~mm} \mathrm{~s}^{-1}$ and although a constant penetration rate was set (through manipulation of the hydraulic spool-valve on the tractor) the actual rate sometimes varied by $\pm 1 \mathrm{~mm} \mathrm{~s}^{-1}$ as hardness varied, with the cone slowing or accelerating upon entering or leaving a harder layer. Figure 5.15 shows depth-averaged tip resistance for each test versus penetration rate; the solid line is a power-law fit with $\mathrm{R}^{2}=0.97$.

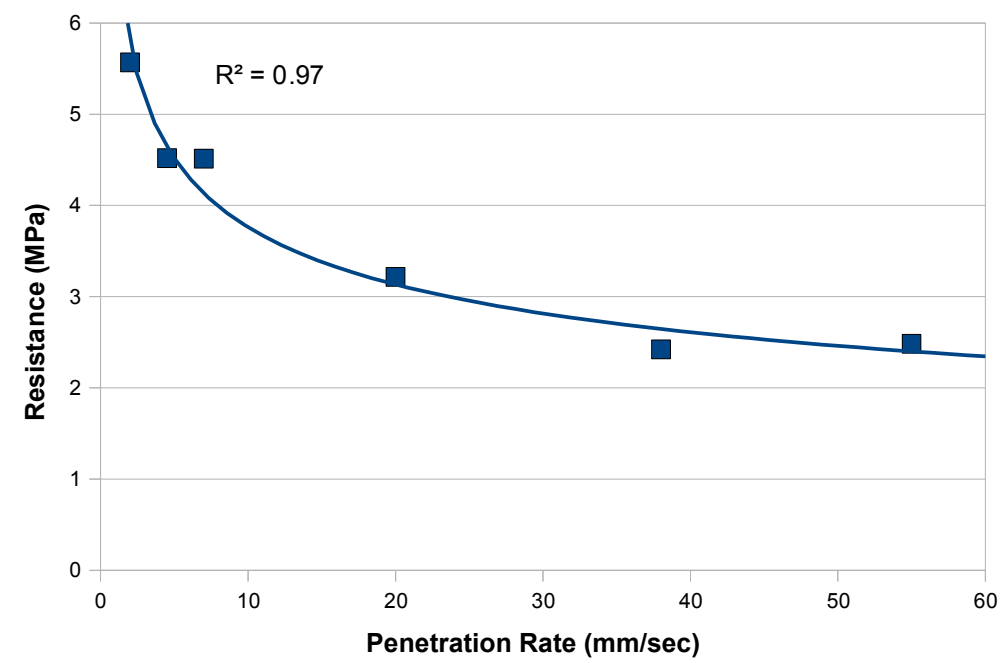

Figure 5.15: Variation of mean cone tip resistance with penetration rate; values are significantly different $(\mathrm{p}<0.01)$.

Applying the one-way ANOVA (analysis of variance) test to the variable-rate CPT data used to generate Figure 5.15 shows that the mean resistance values are not statis- 
tically equal (F statistic $=\sim 150, \mathrm{p}<0.001$ ) and the Tukey HSD test confirms that all mean resistance values are significantly different from one another $(\mathrm{p}<0.01)$. Figure 5.16 suggests that a rate effect is also apparent when measuring snow friction via a sleeved penetrometer.

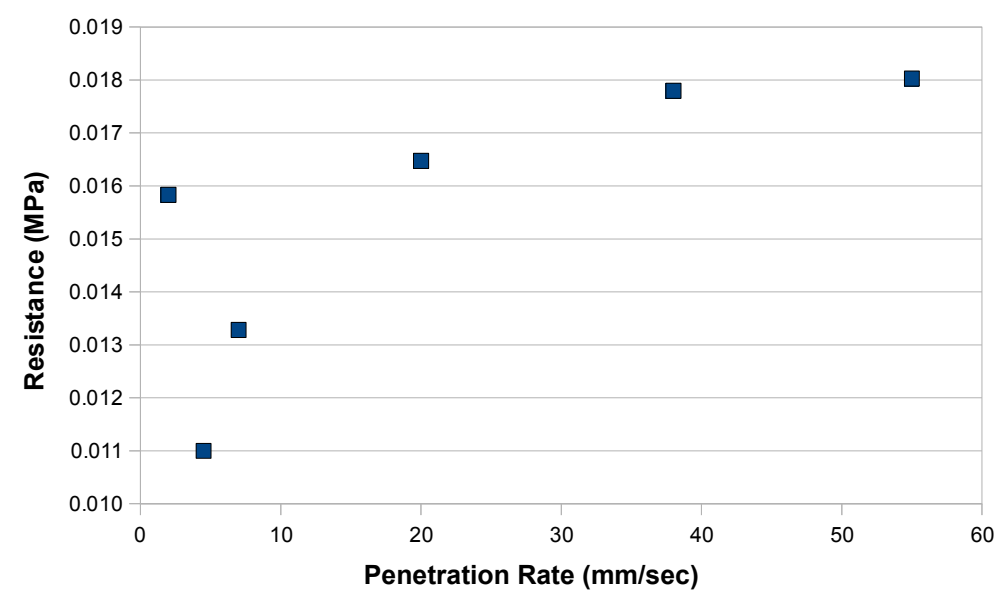

Figure 5.16: Variation in mean sleeve friction with penetration rate.

This figure almost mirrors Figure 5.15, except for the initial data point at a rate of $2 \mathrm{~mm} \mathrm{~s}^{-1}$. If this data point is removed as possibly erroneous, then a power-law curve $\left(\mathrm{R}^{2}=0.94\right)$ is also seen to fit the data well (Figure 5.17; line of best fit extends to zero, graph is truncated for display).

Applying the one-way ANOVA (analysis of variance) test to the variable-rate friction data used to generate Figure 5.17 shows that the mean resistance values are not statistically equal ( $\mathrm{F}$ statistic $=\sim 27, \mathrm{p}<0.0001$ ) and the Tukey HSD test confirms that all mean resistance values are significantly different from one another $(\mathrm{p}<$ 0.01). This suggests an inverse relationship between tip resistance and sleeve friction at varying penetration rates.

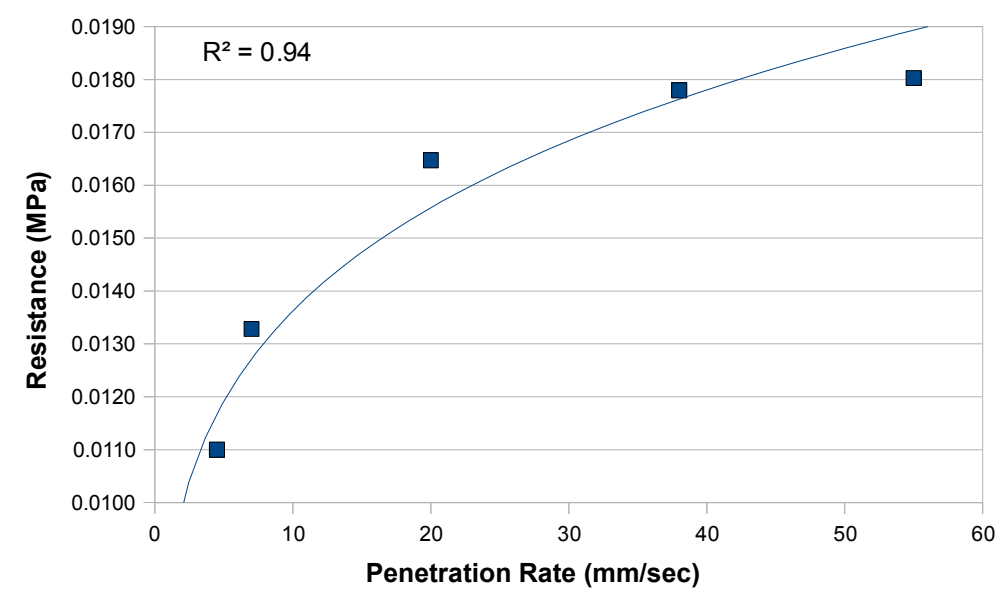

Figure 5.17: Variation in mean sleeve friction with penetration rate (possibly erroneous point removed); values are significantly different $(\mathrm{p}<0.01)$.

Note that the use of $\mathrm{R}^{2}$ as a measure for variance within a power law fit is problem- 
atic. Such a fit is typically estimated by performing a least-squares linear regression on the linear fit of the data upon a log-log plot. The variance from this linear fit line $\left(\mathrm{R}^{2}\right)$ is taken as an indicator of the quality of fit (Clauset et al., 2009). However, this linear approximation induces errors which are additionally harder to estimate, and distributions that are nothing like a power law can appear to follow a power law when a linear log-log fit is applied to such a small sample size; sample sizes where $n \leq \sim 50$ should be treated with caution (Clauset et al., 2009). Therefore, the most that should be said about the apparent high values for $\mathrm{R}^{2}$ noted both above and further within this work is that power-law behaviour may be evidenced by such a value, but such a fit is not necessarily representative or accurate. Ideally other statistics such as the Kolmogorov-Smirnov (KS) statistic (Press et al., 1992) should be applied. However, the limited sample size prohibits ready application of this or similar techniques. The observed variation in both tip resistance and sleeve friction with rate is now discussed.

\subsubsection{Tip Resistance}

Johnson and Schneebeli (Johnson and Schneebeli (1998), Schneebeli and Johnson (1998), Johnson and Schneebeli (1999), Schneebeli et al. (1999)) have performed extensive investigations into penetration in snow but do not comment on the effect of penetration rate on tip resistance measured by the SMPT, merely saying that "Constantvelocity penetration $\left(1-40 \mathrm{~mm} \mathrm{~s}^{-1}\right)$ avoids the rate-dependance associated with creep deformation."

Kinosita (1967) in his compression tests on snow found that, once in the brittle zone, stress decreased as speed of compression increased, initially rapidly and then more gradually, almost asymptotically. Gubler (1975) during Rammsonde tests showed that hardness decreased substantially as penetration rate increased (within the brittle zone) and Fukue (1977) in tests on a penetrative blade into snow, identified the ductilebrittle transition zone and also noted a decrease in strength at an increased rate of deformation. These findings are not unexpected and are consistent with the brittle behaviour of snow's primary constituent, ice. Variations may be expected owing to the compaction and densification of snow, but if any level of bonded microstructure is present within the snow then it is ice bonds that are failing, hence behaviour consistent with ice is expected.

Andersland and Ladanyi (1994) state that frozen soils are much more rate sensitive than non-frozen soils and Ladanyi et al. (1991a) (in (Lunne et al., 1997)), commenting on rate controlled cone penetration tests in permafrost and ice, suggest that a powerlaw relationship can be used to express the relationship between penetration resistance $\mathrm{q}_{c}$ and penetration rate, $\nu$ :

$$
q_{c}=q_{o} \frac{\nu^{\frac{1}{n}}}{\nu_{o}}
$$


where $n$ is the creep exponent and $q_{o}$ and $\nu_{o}$ are the coordinates of any selected point on the straight line plot of $q_{o}$ and $\nu_{o}$ on a log-log scale. This equation is derived from tests in the ductile zone, but because rate effects in the brittle zone also appear to follow a power-law relationship, it can also be applied to the brittle testing conducted at Halley (although $n$ is no longer a 'creep' exponent). This allows estimation of CPT resistance values at various rates within the brittle regime. Buteau et al. (2005) in more recent $\mathrm{CPT}$ in permafrost also found a rate-dependency (within the ductile range), hence stress dependency upon rate is apparent in snow, ice and other frozen geomaterials.

Contrary to these findings, Floyer (2008) in his $\mathrm{PhD}$ thesis investigating penetration of a $12 \mathrm{~mm}$ rounded, manually driven penetrometer "found no substantial dependence of the force-response on velocity over the velocity range tested" (velocities up to 120 $\mathrm{cm} \mathrm{s}^{-1}$ ), a finding further supported by additional work in 2010 (Floyer and Jamieson, 2010) where again they concluded that for a round-tipped $12 \mathrm{~mm}$ diameter penetrometer in uniform snow, no substantial dependence of the force-response on velocity over the velocity range tested was found. Discussions with J. Floyer, 27 May 2009, on this matter concluded that rate effects may play a part in some types of snow and with different penetrometer configurations. Some additional reasons why Floyer and Jamieson (2010) may have found these results are:

1. all their tests were at much higher penetration rates (velocities up to $120 \mathrm{~cm} \mathrm{~s}^{-1}$ versus my maximum penetration rate of $\sim 6 \mathrm{~cm} \mathrm{~s}^{-1}$ ) where the variation in stress with strain rate may be observed to be diminished (see Figure 5.15), and

2. actual strain rates may be much higher and therefore also at the far end of the stress/penetration-rate curve (Figure 5.15), especially noting the rounded penetrometer which may be expected to cause greater snow disturbance than a 'sharp' penetrometer in dry snow.

If measured resistance in snow is rate dependant as I surmise, and the rate curve gradient approaches zero with increasing strain rate once in the brittle zone (Figure 5.15 and work by Schulson (2001) amongst others) then it may be that the penetration/strain rates examined by Floyer and Jamieson (2010) are sufficiently high such that change in measured resistance may appear insubstantial. This is consistent with observation by Kinosita (1967) that at very high rates within the brittle zone, the curve asymptotically approached a constant stress. Conversely, my tests (where rate dependence is evident) were conducted at penetration rates (and possibly strain rates) only just within the brittle regime, where greater deterioration in measured resistance with increased strain rate may be expected. Thus the observed variation of CPT tip resistance with rate is judged to be real. The variation of sleeve friction with penetrative rate is considered next. 


\subsubsection{Sleeve Friction}

In the previous chapter limited data from Greenland suggested that sleeve friction possibly decreased with increased penetration rate, and these data were observed to be consistent with Colbeck (1992). Data from Antarctica presented earlier (Figure 5.17) from more extensive testing suggested that sleeve friction may increase (possibly to a limit) with penetration rate. Further discussion on this is warranted.

Bowden (1953) observed a marked decrease in friction under sled runners as speed increased (up to $5 \mathrm{~m} \mathrm{~s}^{-1}$ ) although a reduced decrease was observed at the speed range of my cone penetration testing $\left(\sim 1-50 \mathrm{~mm} \mathrm{~s}^{-1}\right)$. The mechanism at play was assumed to be lubrication by a water film generated via frictional heating. Mellor (1964) states that in general, friction decreases as sliding rate increases, again typically because of frictional melting and lubrication. No (expected) pressure melting or refreezing was evident throughout the CPT, raising the question as to whether snow dryness and slow penetrative speeds may have contributed to minimal or negligible free water being generated to reduce sleeve friction.

Klein (1947) (in Colbeck (1988)) suggests that there are three components to snow friction: solid-to-solid friction, lubricated friction and capillary suction friction, all of which depend on the prevailing snow crystal type as well as the temperature and liquid-water content of the snow. Solid-solid interaction resulting in high friction will occur when the thickness of any generated water film is insufficient to prevent particle contact. If the nature of the snow tested at Halley was dry enough and cold enough to prevent any free-water forming at tested penetration speeds, then only solid to solid interaction may have occurred.

Bowden (1953) suggests that (for plastic skis) at a temperature of $-10^{\circ} \mathrm{C}$, an increase in friction with rate occurs at speeds below $\sim 100 \mathrm{~mm} \mathrm{~s}^{-1}$. All CPT was conducted below this speed, hence this is the realm of interest. Colbeck (1988) examining this work suggests that at sub-freezing temperatures $\left(0^{\circ} \mathrm{C}\right.$ to $-10^{\circ} \mathrm{C}$, my test temperatures $)$, at rates below Bowden's transition rate (Bowden, 1953), a minimum in friction coefficient is observed, such that there is a zone where friction will rise as speed increases. This may explain the shape of the curve generated via my variable rate friction sleeve measurements (Figure 5.17). This behaviour is further verified by Shimbo (1972) who also found that the coefficient of (kinetic) friction increased towards the static value at speeds less than $0.1 \mathrm{~m} \mathrm{~s}^{-1}$.

The discussion thus far has concentrated on penetration rate per se, and has neglected any possible influence that rate may have on normal stress, a major determinant of friction; friction $(F)$ equals the friction coefficient $(\mu)$ multiplied by the normal force $(N)$. In all tests conducted, fractured particles are considered to have been forced into the hole wall at the cone shoulder, thus contributing to the ensuing friction measurement. The question is whether there is any difference in the residual hole wall density after completion of a test at different rates? Potentially, a slower test allows greater 
sintering and compaction of the side walls to occur, whereas in a faster test perhaps less compaction and densification is possible, resulting in less compaction around the sleeve, increased normal force, and greater friction.

Further work is required to assess the contributing factors towards the observed increase in sleeve friction with penetration rate. This increase, although contrary to general behaviour surmised from the literature is seemingly consistent with data obtained from tests at similar temperatures and rates. Limited frictional melting and variable normal forces on the penetrometer friction sleeve may each play their part in the observed variation of sleeve friction with penetration rate.

\subsubsection{Friction Ratio}

Because the friction ratio is a product of both tip resistance and sleeve friction, its behaviour will be affected by each of these components. The magnitude of tip resistance change due to rate effects is two orders of magnitude greater than supposed changes to sleeve friction, hence it is expected that calculated friction ratios will vary in a form consistent with the tip resistance versus penetration rate data previously presented.

\subsubsection{Summary}

Empirical evidence suggests that CPT data varies with rate within the brittle zone. Although the reason for such differences is not elaborated on here, Schulson and Duval (2009) suggest that such behaviour may be because of strain-rate softening due to fracture processes dominating creep processes as strain rate increases. This subject is briefly inspected again in Chapter 8.

\subsection{Effect of penetrometer size}

Tests 21 - 23 were conducted to investigate whether altering penetrometer size would result in a change in measured tip resistance. Only one size cone was available hence these tests compare resistance from different sized flat plates (Figure 5.18); a cone test is also shown for comparison. Depth is plotted on the $\mathrm{x}$-axis and mean resistance values (horizontal lines) are also presented. Large plate data has been normalised by eleven; the area of the large plate divided by the area of the small plate.

A size effect is evident from these tests: penetrometer resistance decreases as penetrometer size increases. The difference between all tests is statistically significant $(\mathrm{P}$ $<0.0001$ for two-tailed unpaired t test). The normalised average percentage decrease in resistance is $16 \%$ for a real plate area increase of $\sim 1100 \%$.

This decrease is qualitatively consistent with work by Johnson (2003) using smaller penetrometers who showed decreased resistance as penetrometer base area increased (although Johnson showed a greater effect), and is also quantitatively similar to work by 


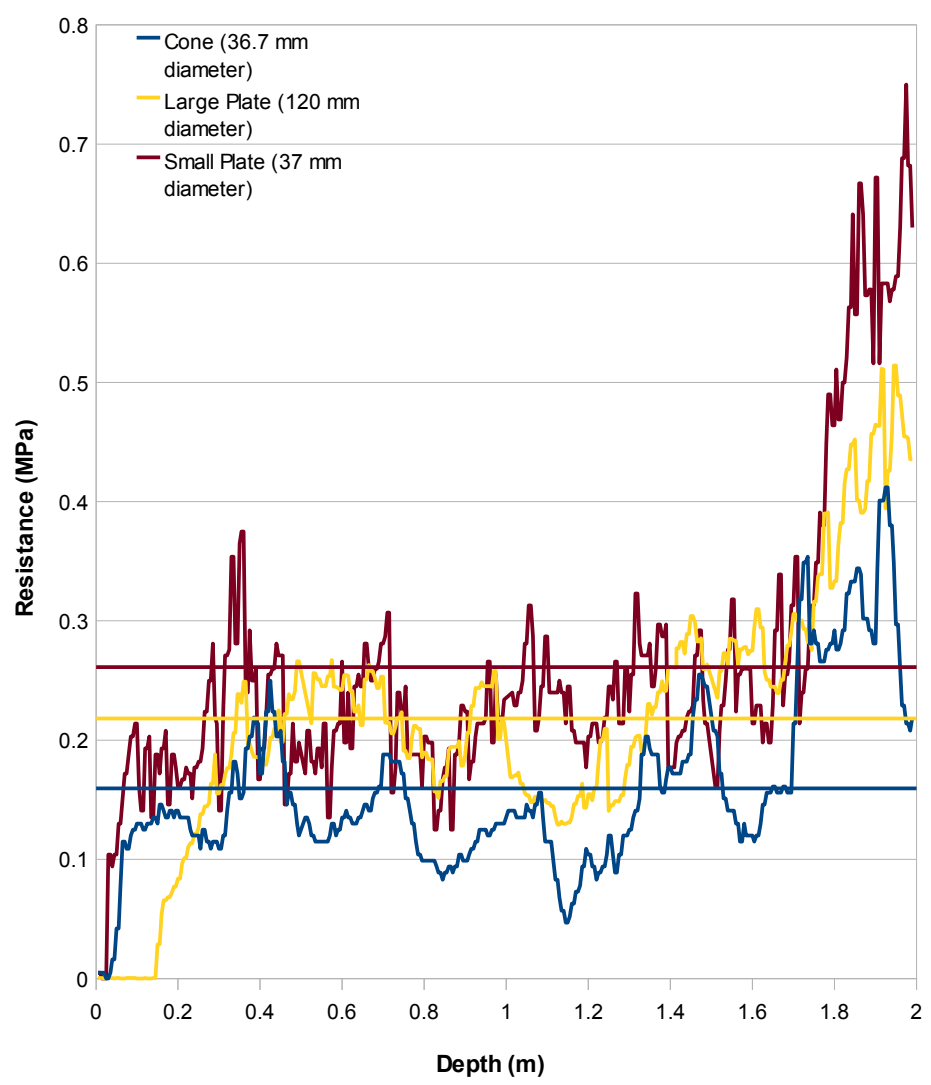

Figure 5.18: Variation in normalised tip resistance between cone and different size plates shows that normalised resistance decreases as penetrometer size increases.

Whiteley and Dexter (1981) who for a similar size increase in area suggest a resistance decrease of $\sim 10 \%$. However, their work was on smaller cone penetrometers with a 30 degree tip angle, and not on flat plates. Lunne et al. (1997) suggest that as the material particle size increases relative to the cone diameter then the cone resistance can increase markedly; this also substantiates an increase in resistance as relative penetrometer area decreases.

Marshall (2005) notes that the size effect is most pronounced when penetrometer size is $<40$ times grain size (average observed in snow pits was $0.7 \mathrm{~mm}$ ) hence this may explain why differences observed were not as great as those suggested by Johnson (2003); flat plates used in testing were of diameter $\sim 37 \mathrm{~mm}$ and $\sim 120 \mathrm{~mm}$. Cone size can also affect local strain rate at the head of the cone, discussed next.

\subsubsection{Cone size and strain rate}

Cone size can affect the rate at which local material is strained at the head of the cone, causing variations in resistance at constant rates of penetration. Ladanyi (1982) exploring the link between probe size, strain rate and tip resistance in frozen geomaterials derived Equation 5.2 to relate representative strain rate $\left(\dot{\epsilon}_{r e p}\right)$, failure strain in uniaxial compression $\left(\epsilon_{f}\right)$, penetration rate $(\dot{p})$ and penetrometer diameter $(d)$ : 


$$
\dot{\epsilon}_{r e p}=6 \epsilon_{f} \frac{\dot{p}}{d}
$$

and then used the following equation (Equation 5.3) to relate strain rates to tip resistance:

$$
q_{c 1}=q_{c 2}{\frac{\dot{\epsilon}_{c 1}}{\dot{\epsilon}_{c 2}}}^{\frac{1}{n}}
$$

where $q_{c 1}$ is tip resistance for penetrometer one, $q_{c 2}$ is tip resistance for penetrometer two, $\dot{\epsilon}_{c 1}$ is strain rate for penetrometer one, $\dot{\epsilon}_{c 2}$ is strain rate for penetrometer two and $\mathrm{n}$ is the creep exponent obtained from the slope of $\mathrm{q}_{c}$ vs strain rate in a log-log plot. (As noted earlier, $\mathrm{n}=\sim 3$ for ice at low strain rates, but may be of the order $10-20$ at higher strain rates.)

Applying these equations to the flat plate data reveals that for $\mathrm{n}=10.3$ (derived from strength of ice vs strain rate, (Petrovic, 2003)), then a reduction in resistance owing to cone size of $14 \%$ is predicted. This agrees very well with the observed average decrease of $16 \%$. A size effect is evident in assessing strength of snow via penetrometer, and further techniques must be explored to enable consistent comparison between data sets from penetrometers of different size.

\subsection{Effect of cone shape}

In Chapter 3 it was briefly noted that variations in cone shape have been shown to affect measured resistance values, both in snow and soils. In order to verify this phenomenon some tests were conducted with a flat plate of identical diameter to the standard $60^{\circ}$ cone; no other cone shapes were used. Figure 5.19 shows the variation in resistance obtained in Tests 9 and 10, located $100 \mathrm{~mm}$ apart.

Resistances are substantially greater in the flat-plate test (statistically significant with $\mathrm{P}<0.0001$ via unpaired t test); the difference in averaged resistance (to $5 \mathrm{~m}$ depth) is $\sim 188 \%$, although the difference at discrete depths sometimes rises to greater than $200 \%$. This is quantitatively consistent with modeled data presented by Johnson (2003) who suggested an increase in resistance of 2 - 3 times as cone angle increases from 60 degrees towards 90 degrees. It is also consistent with this qualitative supposition (Johnson, 2003): that as the tip angle approaches that of a flat plate (a $180^{\circ}$ cone), the increased dimension normal to penetration and the formation of a compacted zone moving ahead of the penetrometer increase the number of snow microstructural elements mobilised and markedly increase resistance to penetration. Gill (1968) on work in soil found similarly, that the measured resistance increased as cone angle increased, primarily as a product of the cone angle, and Nowatzki and Karafiath (1972) found that at higher relative densities where less packing is available, change in cone angle had a greater effect on cone resistance. 


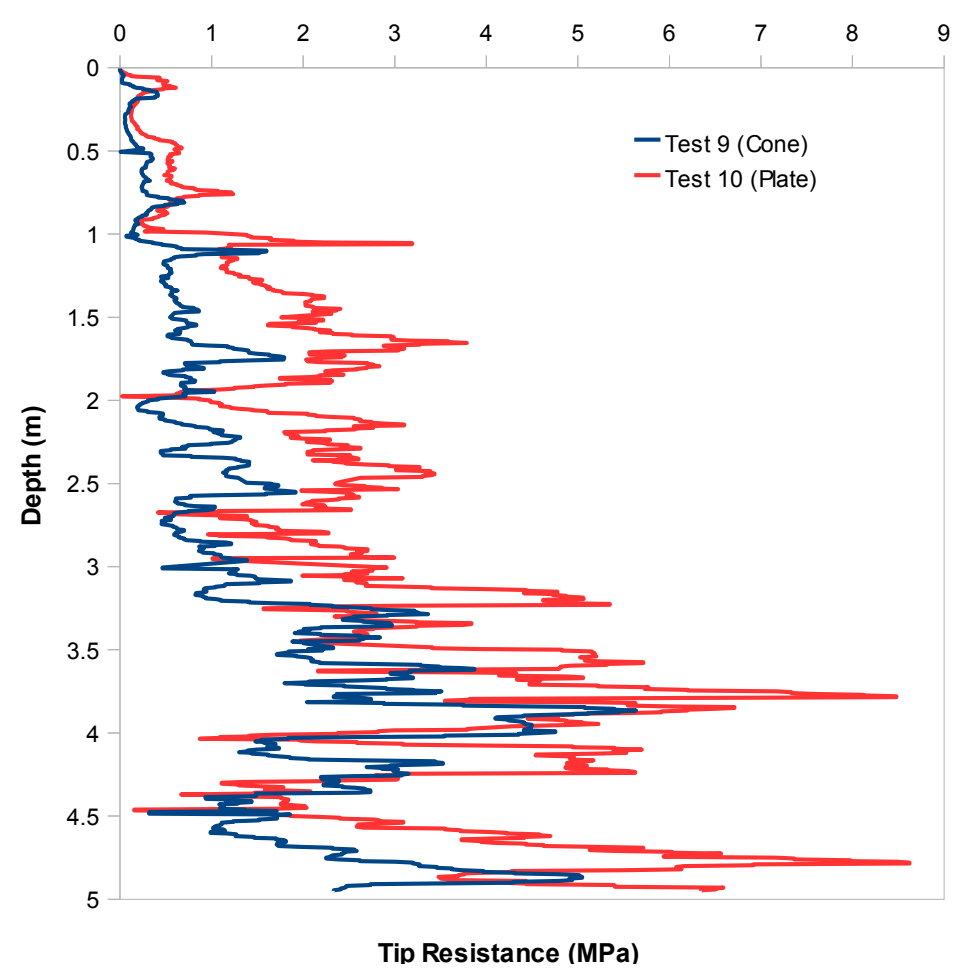

Figure 5.19: Tip resistance versus depth for cone and flat plate. Significantly greater resistance is obtained using a flat plate.

Application of Kinosita's (Kinosita, 1967) equation relating cone angle to penetration resistance in the brittle zone, below:

$$
F=7 \vartheta^{1.67} \rho^{3} Z^{2}
$$

(where $F$ is force in $\mathrm{kg}, \vartheta$ is the vertical angle of the cone (degrees), $\rho$ is snow density $\left(\mathrm{kg} \mathrm{m}^{-3}\right)$ and $Z$ is distance of the cone apex under the snow surface $\left.(\mathrm{cm})\right)$ suggests that variation between the two shapes in snow of density $450 \mathrm{~kg} \mathrm{~m}^{-3}$ may be of the order $\sim 600 \%$. This is far greater than that observed. Reasons for this discrepancy may be:

1. Kinosita's work was essentially shallow penetration where the cone did not penetrate beneath the surface and substantial ejection of overburden upon cone entry was incorporated, and

2. Kinosita's maximum rates of penetration $\left(0.55 \mathrm{~mm} \mathrm{~s}^{-1}\right.$ were only just within the brittle zone ( $>\sim 0.16 \mathrm{~mm} \mathrm{~s}^{-1}$ as defined by Gameda et al. (1996)) where changes in rate result in greater stress variation.

I surmise that during a test with a flat plate the increase in resistance may decrease with depth as compacted material forming on the plate alters the plate shape towards conical. Although perhaps a crude measure, an attempt to assess this is presented in Figure 5.20 which shows the 100 point running mean for the ratio of tip resistance from 
a flat plate test (Test 10) to tip resistance from a standard cone test (Test 9) versus depth.

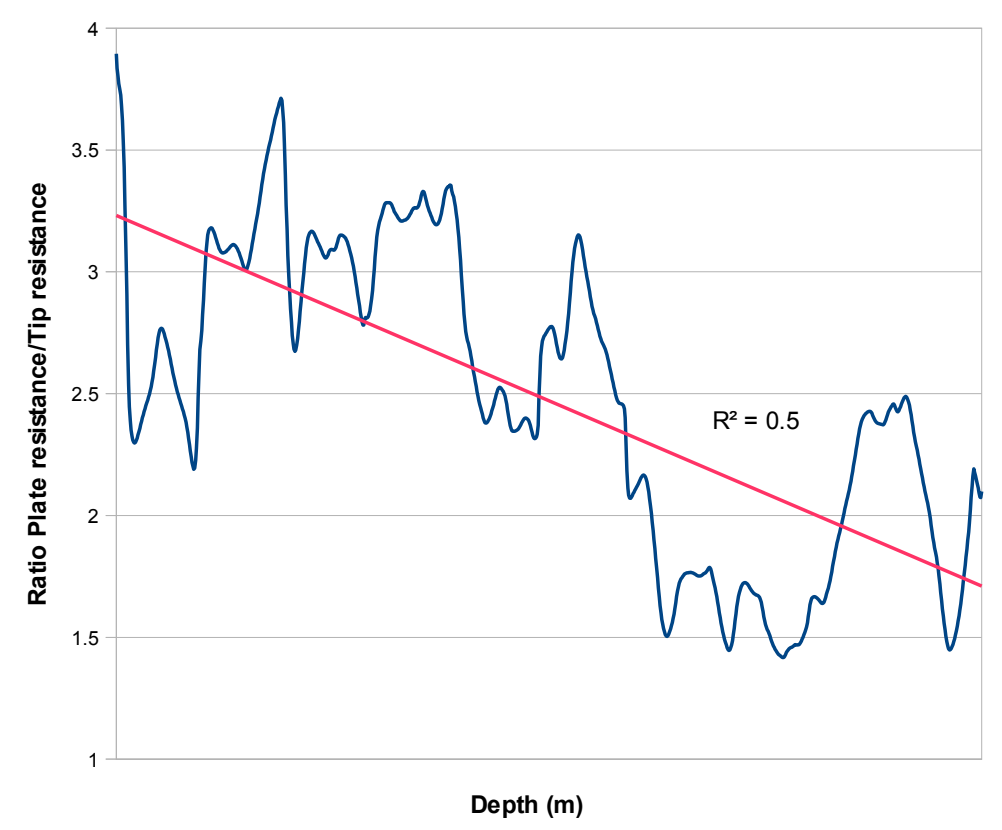

Figure 5.20: Ratio of flat plate resistance to cone resistance generally decreases with depth consistent with the formation of a conical mass of compacted snow on the flat plate as the test progresses.

Although the mean ratio still exhibits large variability owing to stratigraphy, the linear trend line suggests a decrease in this ratio with depth. This suggests that the flat plate may become more 'cone-like' as a test progresses. Further testing is necessary to verify this behaviour, yet it appears intuitively feasible.

The shape of the cone has a marked effect on the physical interaction of the penetrometer with the medium with resultant effect on measured resistance values and must be considered when attempting to compare or interpret penetrative tests.

\subsection{Cone Effective Area}

Similar to the testing conducted with the Fugro mini-penetrometer, some tests were conducted in an attempt to verify the effective area of the cone; such variation may affect calculated resistance values. Although limited accuracy of measuring equipment and the numerous parameters affecting post-test hole size precluded accurate quantitative assessment of the cone effective area, it is acknowledged that the cone likely bears upon a surface area greater than that of itself whilst undergoing penetration, hence this topic is discussed a little further.

Mellor (1975) in discussion on Rammsonde penetration resistance notes that the effective area of a penetrometer in snow is expected to be greater than the actual penetrometer area and will increase with increasing density. Work by Mulqueen et al. 
(1977) (reported in (Shoop, 1993)) on cone penetration testing in soil revealed compacted soil ahead of the cone effectively changing the cone's size and shape, and Johnson and Schneebeli (1998) in their patent for the Snow Strength Penetrometer (described within this work as the Snow Micropenetrometer (SMPT)) make the statement that for large penetrating cones a "snow body forms on the cone" and "the shape of the penetrating tip changes."

The establishment of a 'snow body' or 'compacted zone' moving ahead of an advancing cone is discussed next, but it does appear that some level of additional bearing area (effective area) commensurate with snow grain size is probably established as a cone is driven into snow. This impact may be up to $\sim 7 \%$ of the measured cone resistance, based upon a displaced grain size of $0.7 \mathrm{~mm}$, and a cone diameter of $36.7 \mathrm{~mm}$. The size of the effective area is dependant on the nature, density and grain size of the snow and the size, shape and composition of any penetrometer. It is a small effect but consideration appears appropriate when interpreting cone resistance data.

\subsection{Formation of compacted zone}

Qualitative assessment was attempted to evaluate whether a compacted zone of fractured snow was formed and pushed ahead of the advancing cone or plate. Both still and video footage of an advancing cone and plate, plus examination of CPT holes post-test were used in assessing this phenomenon, as described below.

In order to try and observe the physical processes as the cone penetrated the snow pack, a test was conducted at a slow rate, and then an incision was made in the snow pack parallel to the cone to enable observation of the cone tip. This enabled the advancing cone to be filmed (see Figure 5.21 for a screen shot).

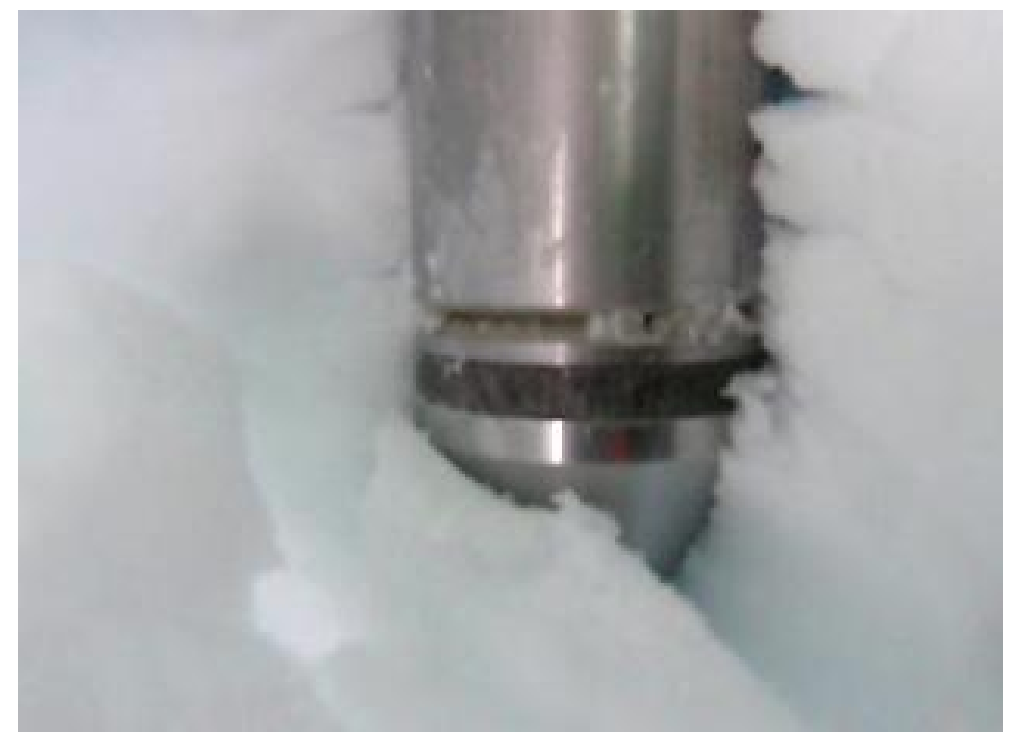

Figure 5.21: Screen shot of a $36.7 \mathrm{~mm}$ diameter standard cone slowly advancing in cut-away hole. 
Although by forming such an incision the behaviour of the snow was compromised, some observation of what was happening at a granular level was possible. Snow grains appeared to be displaced in a direction largely perpendicular to the advancing cone face (consistent with the work in agricultural soils by Whiteley and Dexter (1981) and the statistical-micromechanical model proposed by Johnson (Johnson, 2003) amongst others) as noted previously, before being forced into the sides of the bore hole at the cone shoulder. The outcome of this process can be observed in Figures 5.22 and 5.23.

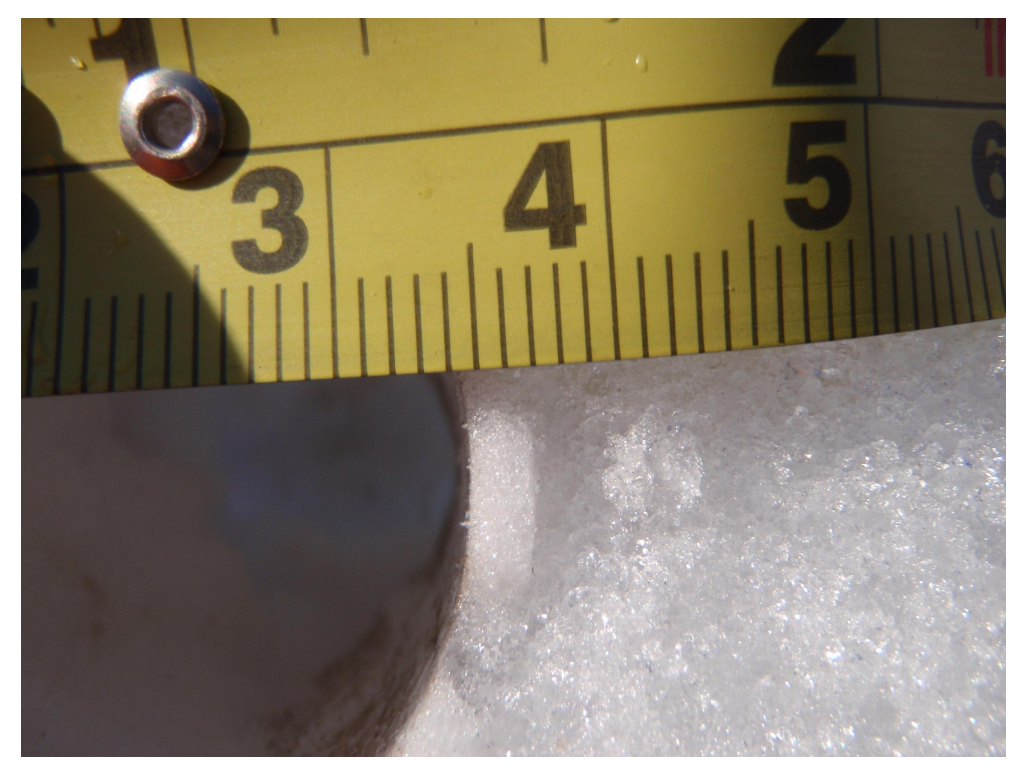

Figure 5.22: Compacted perimeter ( $\sim 2 \mathrm{~mm}$ thick, tape for scale) observed in crosssection of hole post-test. Fractured material ahead of the cone is compacted to the side forming an annulus.

Figure 5.22 shows an annulus of compacted material observed around the perimeter of the CPT hole post-test, presumed to be formed by the compaction of snow into the wall at the cone shoulder, whilst Figure 5.23 shows the composition of this annulus as an excised portion is held to the sun. The perimeter appears as a bonded denser structure, whilst the surrounding snow remains granular and uncompacted.

These qualitative results suggest that no compacted zone was formed ahead of the advancing cone during CPT testing, rather the fractured material was pushed normal to the sides and forced into the hole walls at the cone-shoulder. The hardness of this annulus of compacted snow rose from 'finger' to 'pencil' post-CPT.

Similar observational techniques were used in attempting to identify the existence of a compacted zone formed ahead of an advancing flat plate. Figure 5.24 shows a cone of compacted snow, routinely observed on the face of the flat-plate upon completion of a test.

Cross-sections of the hole formed during CPT were not examined after any flat-plate tests, so the existence or otherwise of a compacted perimeter is not known. However, it is surmised that after initial formation of a conical plug on the end of the flat plate, the snow again behaves in a similar manner during penetration, being forced to the sides at 


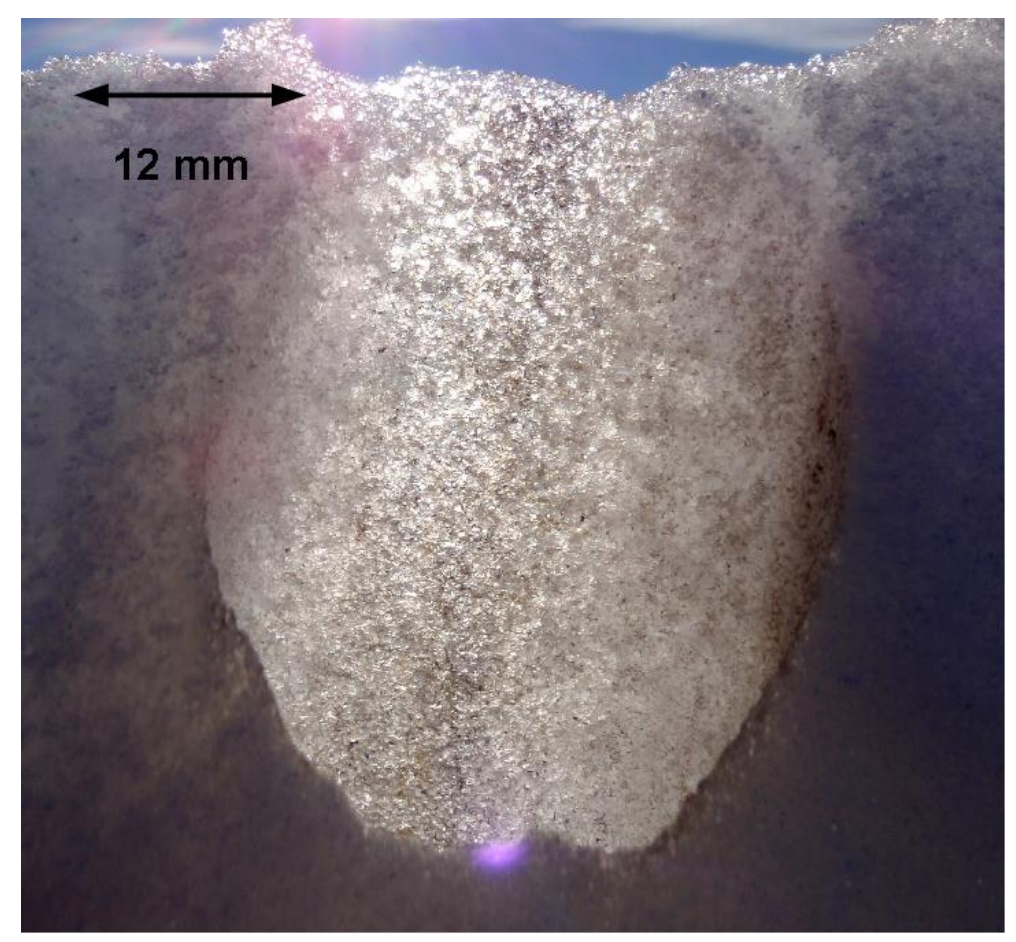

Figure 5.23: Compacted and bonded annulus (light area) surrounded by uncompacted virgin material (dark area).

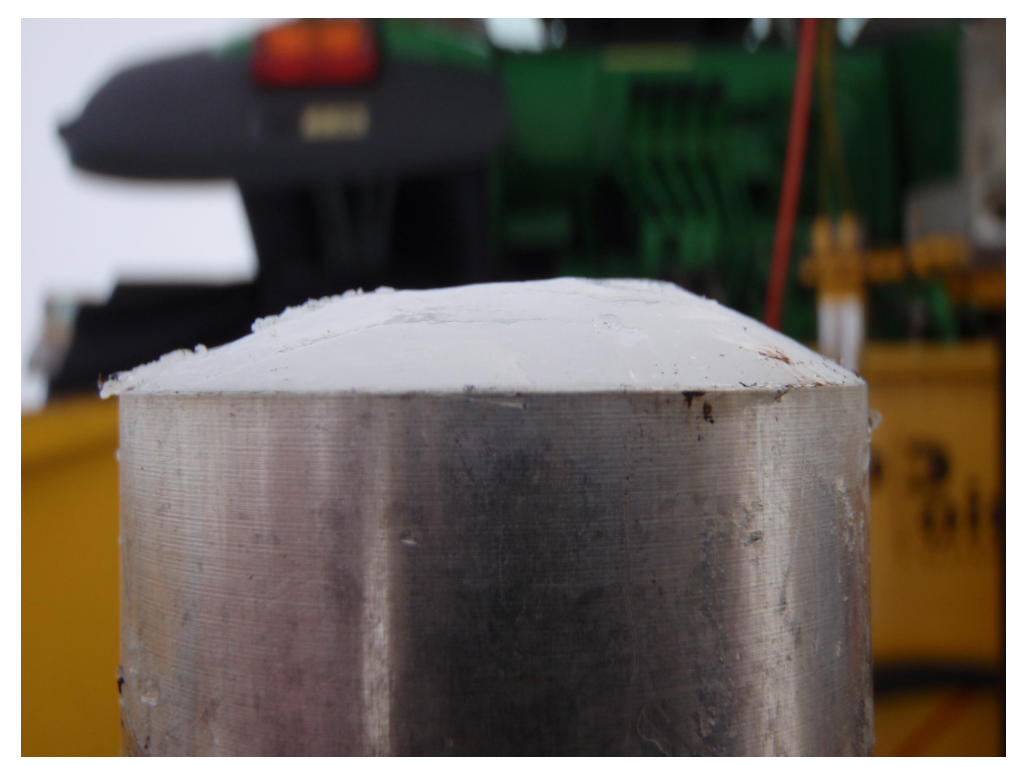

Figure 5.24: Low-angle conical plug routinely observed on flat-plate post-test.

the cone-shoulder, although deformation, destruction, and reformation of the conical plug ahead of the flat plate are likely to occur as the penetration process continues, especially through layers of varying hardness. This phenomenon is further discussed below.

J. Floyer (personal communication, 27 May 2009) clarified that the formation of a compacted zone ahead of a penetrating object in snow will probably depend upon the size and shape of the indentor, the nature of the snow, and probably also the penetration rate. In both the Fugro mini-penetrometer testing and the CPT presented 
herein, the combination of a relatively thin, smooth, sharp tip and extremely dry snow appears to have resulted in an immeasurable or negligible compacted zone ahead of the penetrometer, with all fractured particles forced normal to the penetrometer face and thence compacted into the sides at the cone shoulder. The compaction of fractured material into the sides of the hole at the cone-shoulder appears to be the dominant displacement mechanism occurring ahead of the $60^{\circ}$ steel cone in the dry snow tested and is consistent with Kinosita (1964) who described the packing of broken ice grains into the compressed region beside or under the cone; snow was preferentially displaced to the side as cone angle decreased.

When a flat plate was used, a compacted zone of snow (approaching the density of ice) was formed on the plate during testing. Although the snow in question was again very dry and likely not conducive to bonding, I surmise that fracture, compaction and then pressure-melting of particles occurs ahead of the flat plate. The depression of the melting point of ice varies between $\sim 0.08 \mathrm{~K} \mathrm{MPa}^{-1}$ and $\sim 0.1 \mathrm{~K} \mathrm{MPa}^{-1}$ (depending on the saturation-state of the air) (Hooke, 2005) and pressures during flat-plate penetration approached $10 \mathrm{MPa}$, suggesting that a $1^{\circ} \mathrm{C}$ depression of the ice melting point may be possible. (Note also that actual pressures on the plate face may exceed those recorded owing to the limitations of the CPT data acquisition system.) Snow temperatures were not routinely recorded during CPT but outside air temperatures occasionally reached $>0{ }^{\circ} \mathrm{C}$, hence the existence of some pressure melting ahead of the flat plate can not be discounted. It is surmised that this conical zone formed after a certain distance and probably deformed throughout the test: growing in size, yet also likely deflecting additional particles (because of its conical shape) as penetration continued. The formation of a similar 'body' is reported by Gill (1968) in his work in agricultural soils who says that "the compaction and adhesion of soil to blunt rigid bodies...results in the formation of soil bodies"; when the tool is blunt a clear and distinct soil body forms on the body of an advancing penetrometer.

The formation of compacted material on a penetrating flat plate appears possible and is consistent with observations in other geomaterials. During penetration of a $60^{\circ}$ cone in dry polar snow it appears that fractured material is displaced preferentially to the sides and no compacted zone is formed ahead of the cone. Quantification of stresses realised throughout this process is examined in Chapter 7, and the compaction or densification of the snow alongside the cone also has obvious implications for the friction measurement obtained via the friction sleeve.

\subsection{Sleeve Friction reassessment}

Investigations into the formation of a compacted zone described above suggest that broken material ahead of the cone is forced into the side-walls of the cavity during penetration. In order to try and assess this phenomenon quantitatively, additional 
testing was carried-out in a series of pre-drilled holes. This enabled the comparison of friction in a normal test (where material is presumed to have been forced into the cavity walls at the cone shoulder, thus altering wall density and probably measured friction) with friction in a pre-drilled hole where limited material is forced into the walls at the cone shoulder, thus the original density of the snow is retained to allow a more accurate assessment of friction. This testing method was suggested by B. Norrish, BAS Vehicle and Plant Manager, 27 January 2010.

Tests 38 and 39 were conducted in pre-drilled holes (pre-drilled to $0.5 \mathrm{~m}$ depth using a $32 \mathrm{~mm}$ drill bit) and are compared to two collocated tests (41 and 42) in Figure 5.25. Depth is plotted on the x-axis and mean resistance values (horizontal lines) are also presented.

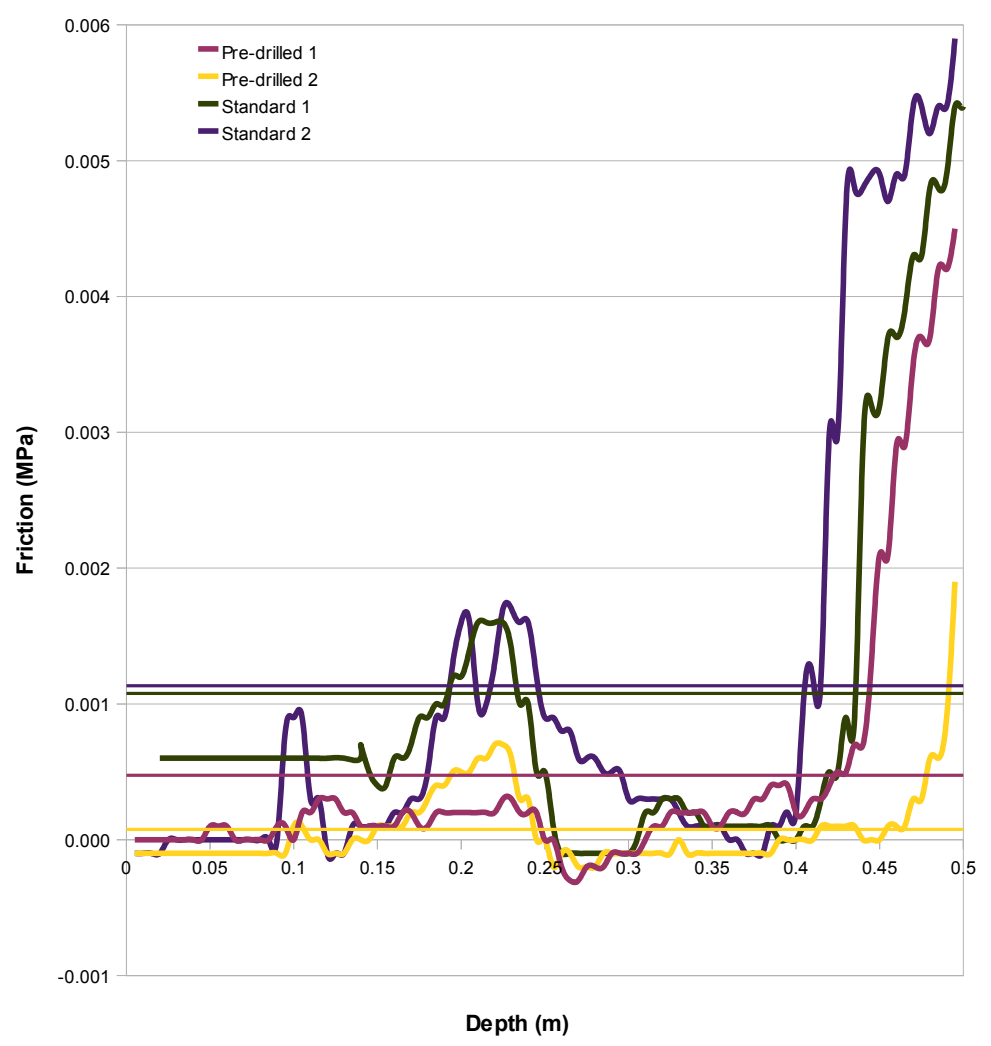

Figure 5.25: Friction variation in pre-drilled holes shows that friction is less when compaction into the sides is reduced, presumably owing to a reduction in normal force acting upon the friction sleeve.

A variation in measured sleeve friction is evident from this figure. The difference within each test-type is not statistically significant, but the difference between the different types of test is statistically different $(\mathrm{P}<0.0001$ for unpaired t test). Thus a difference in average sleeve friction is evident between a standard test and a test in a pre-drilled hole, suggesting that sleeve friction data are affected by compaction of material into the hole annulus. 


\subsection{4 'Sensing' ahead of the penetrometer}

One of the aims of this research was to ascertain whether the cone was 'sensing' ahead of itself during penetration (as occurs in most soils) and thus providing erroneous resistance versus depth information. In order to try and capture this behaviour, standard tests were conducted towards a stiff layer: firstly ice (Tests $50 \& 51$ ), and then a buried metal plate (Tests 55 to 57 ). Tests were conducted using both a standard $60^{\circ}$ cone and a flat plate of similar diameter.

The tests conducted with a cone towards a layer of ice were inconclusive, hence tests 55 to 57 were then conducted towards a buried $2 \mathrm{~mm}$ thick copper sheet. Figure 5.26 shows the results of this testing, with cone tip resistance observed to remain very low through the sugary, low density $\left(350 \mathrm{~kg} \mathrm{~m}^{-3}\right)$ snow at this site (adjacent skiway) before increasing rapidly, apparently upon hitting the buried plate.

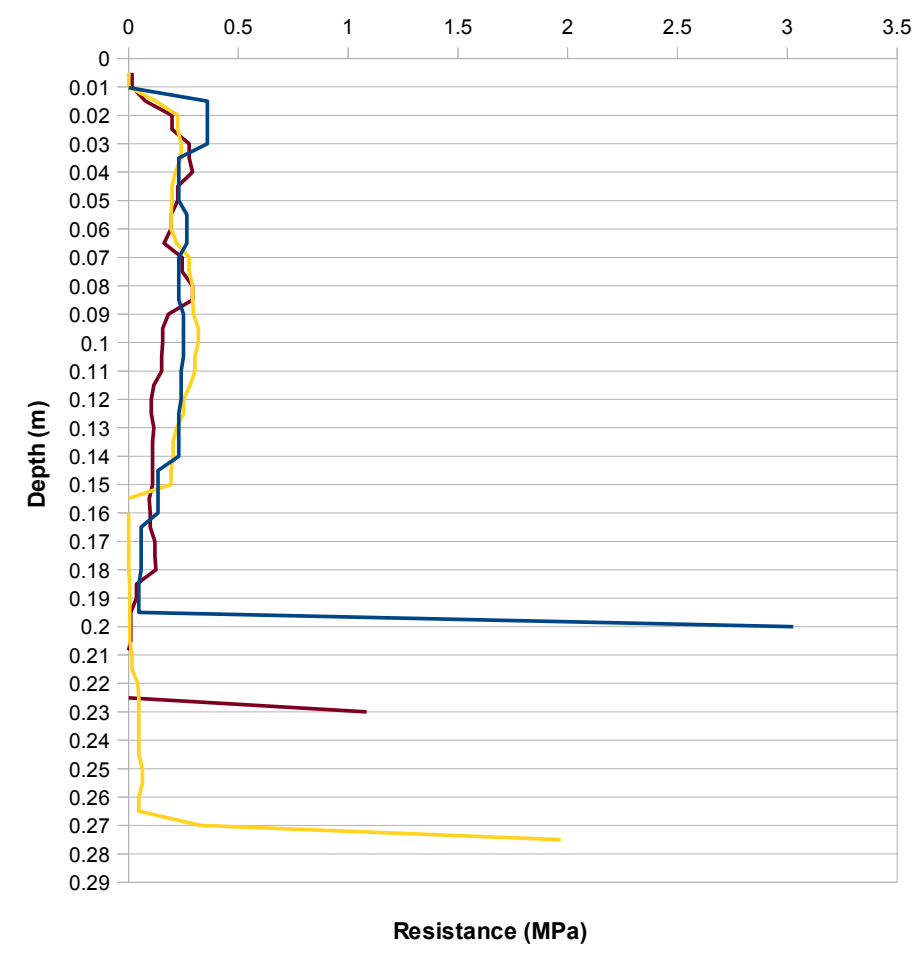

Figure 5.26: Tests towards a buried copper plate show that resistance increases upon impact, but no increase ahead of the impact due to 'sensing' is observed.

Indentation marks confirming impact were observed in the copper sheet after each test and within the limited data-recording capability of the CPT system (recording every $5 \mathrm{~mm}$ penetration) no 'sensing' was evident.

Additional testing on this matter was conducted 'upside-down' by testing whether transmission of pressure could be detected ahead of the advancing cone, rather than whether the cone could sense a future hard surface. To this end, in numerous tests a pressure sensor was buried directly beneath the cone's envisaged path of travel and then a penetration test towards the sensor was conducted, the idea being that the 
sensor may detect the proximity of the cone before any physical impact (of cone or fractured particles) had occurred. In an ideal test if 'sensing' did exist, both the cone and the pressure sensor would 'sense' each others presence some distance before meeting. Unfortunately owing to difficulties in sensor/cone alignment and pressure sensor irresponsiveness and limited cone data acquisition rate, no successful results were achieved via this method.

Only one test was conducted in an attempt to assess sensing ahead of a flat plate (Test 50). This test was conducted towards a known ice layer but results were also inconclusive. The resistance trace increases markedly within $10 \mathrm{~mm}$ of the hard ice layer, but the likely existence of a compacted mass (of dimension $5-10 \mathrm{~mm}$ ) on the front of the plate and the limited data-recording accuracy of $5 \mathrm{~mm}$ do not enable discrimination of any sensing behaviour.

The nature of what exactly cone 'sensing' entails was briefly outlined in Chapter 3. To reiterate: although a zone of compacted material (compacted zone) may or may not form ahead of the cone, sensing is understood to be the detection by the leading edge of the cone (or of the compacted zone ahead of the cone) of future, yet to be penetrated layers via elastic deformation of the material ahead of the cone, thus resulting in force-transferral, but not fracture of these layers or particles. Summative resistance to depth and breadth (depending on load dimensions) via elastic deflection provides the resistance necessary to support the load. This is analogous to the pressure bulb concept and is graphically represented in Figure 5.27 via stylised stress / strain curves at increasing distance from an advancing cone.

In a dynamic situation, the elastic regime of behaviour will rapidly be surpassed and brittle fracture of ice grains and grain bonds will occur. Load will be transferred to grains / bonds distant from the applied load, and elastic deformation will result, but these layers will soon be additionally loaded by the advancing cone / plate (or fractured material) and will themselves fail in a brittle manner, and so the sequence will continue amidst compaction, densification and displacement of fractured particles (behaviour dependant upon cone size / shape and material properties). So 'sensing' relates specifically to the elastic component of resistance experienced by the cone. Additional forces due to compaction of material ahead of the cone are discussed in Chapter 7 .

The magnitude of this elastic component depends upon the Young's modulus (E), a bulk elasticity modulus for the snow that defines the approximate slope of the elastic regime of the stress / strain curve. Theoretically, a value of E can be derived from cone resistance values; this is considered in Chapter 7. Typical values of $\mathrm{E}$ for snow of density $450 \mathrm{~kg} \mathrm{~m}^{-3}$ are $\mathrm{E}=\sim 500 \mathrm{MPa}$, and for ice, E may be of the order of $10 \mathrm{GPa}$ (Mellor, 1975).

Ice generally fails at a strain $\leq 0.003(0.3 \%)$ (Petrovic, 2003) and if an average grain size of $\sim 0.75 \mathrm{~mm}$ (consistent with snow pit data) is considered, then failure of ice bonds 


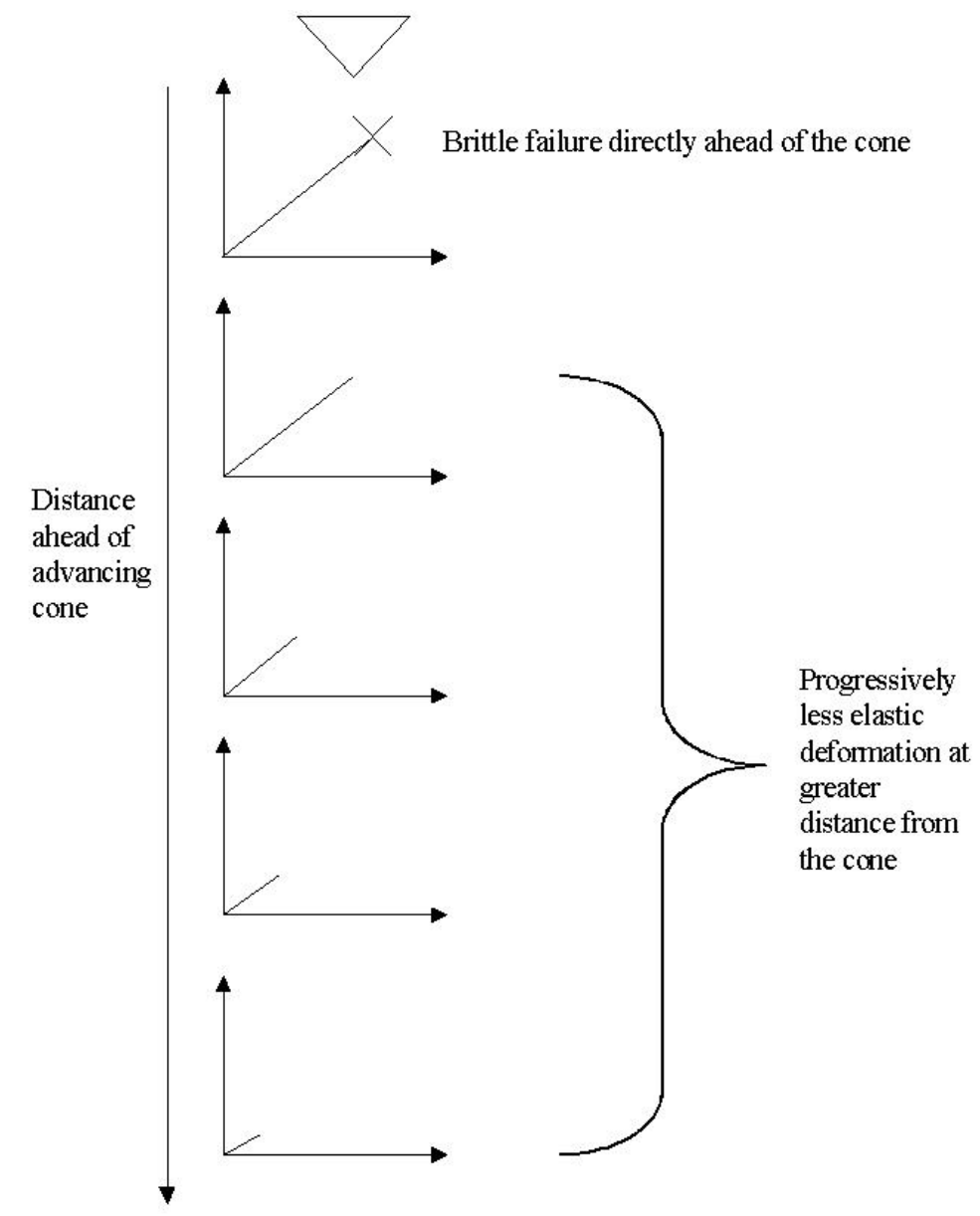

Figure 5.27: Schematic stress / strain curves show that decreased elastic resistance is mobilised at greater distances ahead of the cone.

or grains occurs after elastic deflection in the order of micromillimeters. Thus if the maximum deflection of an ice grain ahead of a penetrometer moving at a rate to cause brittle fracture in snow is of the order, micromillimeter, then the ability to detect any 'sensing' or disturbance of material ahead of the cone via elastic deformation appears negligible, especially noting the limitations of the equipment used. It is therefore not surprising that no sensing was observed ahead of the cone or flat plate during testing.

Sensing may be possible in unbonded snow where particle displacement may be possible without brittle fracture and some resistance ahead of the cone may be incorporated. However, the discussion above suggests it will not occur in bonded snow, or at least will not be detectable via the methods and equipment employed herein.

\subsection{Effect of confining pressure}

In Chapter 3 the effect of overburden pressure or confining stress was briefly outlined. Such an effect appears dependant upon the density of the snow in question and an assessment of the effect in the environs of Halley $\mathrm{V}$ was of interest. In order to verify the effect of overburden pressure, six tests were conducted in close proximity (in an attempt 
to reduce spatial heterogeneity) with a layer of surface material removed between each test, as shown in Figure 5.28.

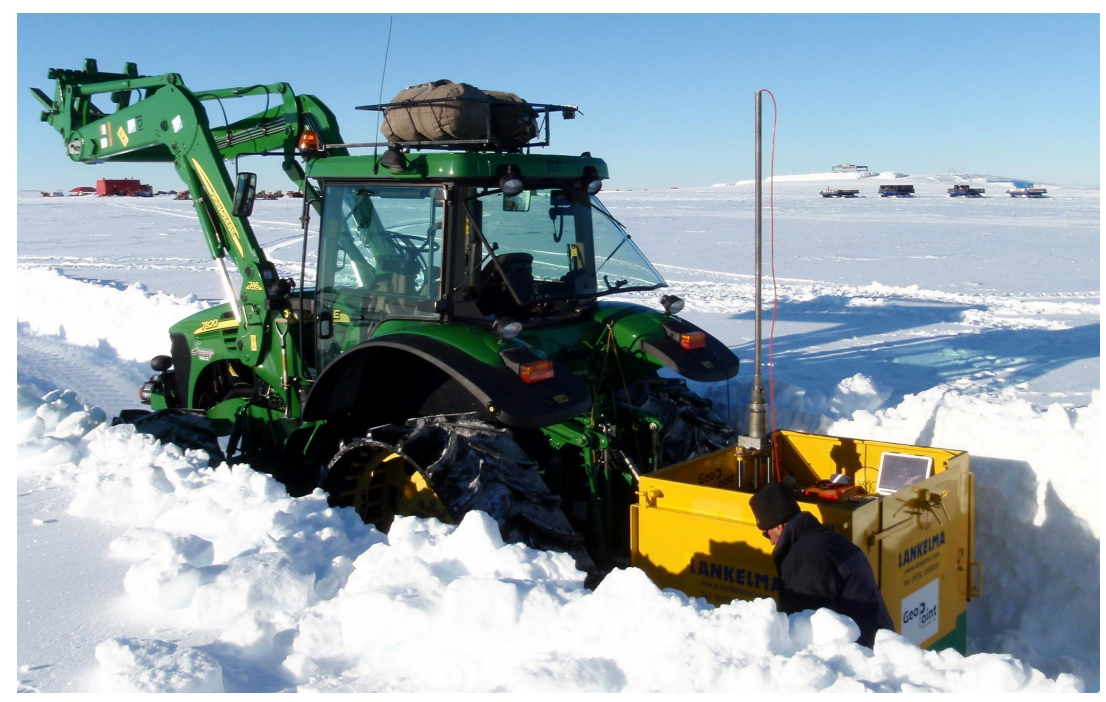

Figure 5.28: Overburden was sequentially removed by bulldozer to enable assessment of resistance variation under varying confining pressures.

Note that experimental technique may have affected these test results because although no obvious deep fracture or compaction was observed, a bulldozer was used to remove the substantial volume of overburden, with typically two to three passes required each time. Such traffic will have had some impact in compacting and deforming the snow pack prior to later tests on supposedly less-confined material.

Two tests using a standard cone at $25 \mathrm{~mm} \mathrm{~s}^{-1}$ penetration rate were then conducted under each amount of overburden. The effect on both tip resistance and sleeve friction is now considered.

\subsubsection{Tip Resistance}

Variations in tip resistance over the same depth range of snow for Tests 80 - 85 are shown in Figure 5.29 with a line of best fit of gradient $-0.11 \pm 0.078$; each point on the graph is the mean of 196 to 212 data points.

Variations in mean tip resistances at different overburden pressures are not consistently statistically different, therefore it would appear unwise to draw firm conclusions on the effect of overburden pressure on cone tip resistance.

The rationale for a possible increase in resistance as overburden is increased, is that crack initiation is restricted and greater resistances can be achieved (Renshaw and Schulson, 2001). In this experiment the variation in confining stress is very small (see Figure 5.10) compared to tip resistances hence an insignificant or unclear trend as evident is not unexpected. Also, and importantly, in porous (lower density) snow this effect will be significantly reduced as regardless of the confining pressure, sufficient pore space exists to ameliorate this effect, enabling unhindered fracture and compaction 


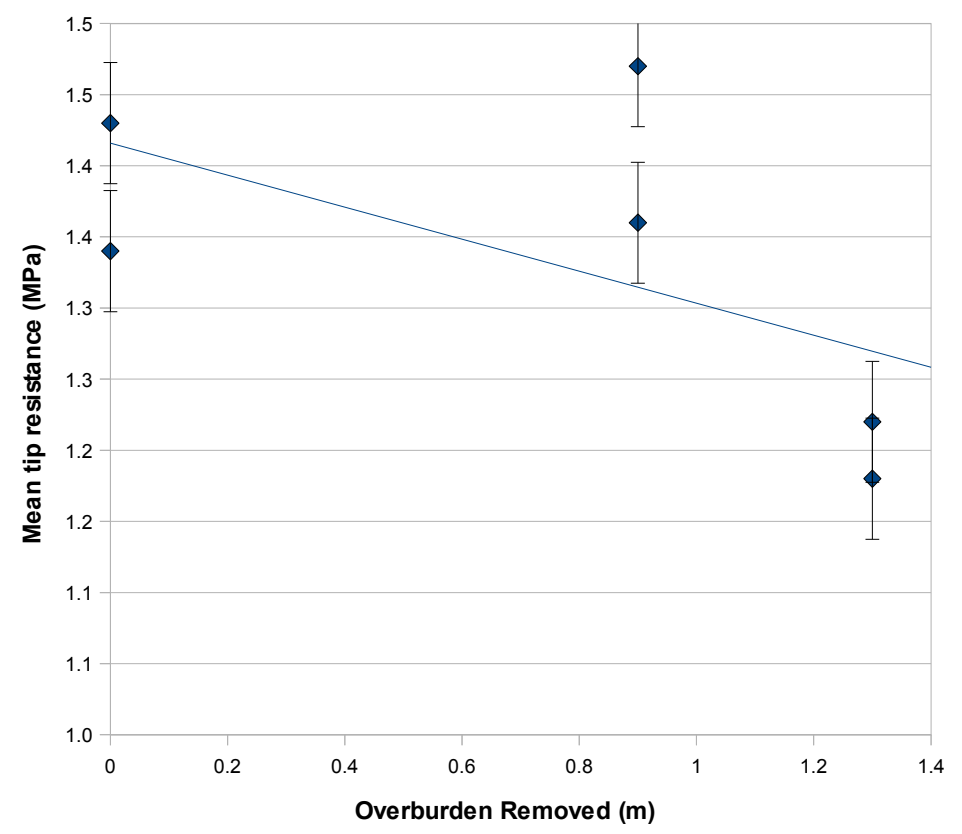

Figure 5.29: Inconclusive variation of tip resistance with amount of overburden removed.

regardless of the applied amount of confining pressure, whereas as porosity reduces (and density approaches that of ice) the effects of confinement may increase.

Therefore, although confining pressure may have some impact upon the tip resistance proffered by polar snow, the variation is complicated by the density and microstructure of the material, and analysis of data is inconclusive.

\subsubsection{Sleeve Friction}

As noted in an earlier discussion on friction variation with depth, it is possible that an increase in overburden stress may have a greater impact on sleeve friction. Variations in sleeve friction for Tests 80 - 85 are shown in Figure 5.30 with a line of best fit of gradient $-0.003 \pm 0.0004$; each point on the graph is the mean of 195 data points.

These data show sleeve friction decreasing as overburden pressure is reduced; an almost $40 \%$ reduction is observed after the removal of $2 \mathrm{~m}$ of overburden. This suggests that overburden may increase the normal stress on the friction sleeve and thus the measured friction. This is consistent with literature describing the evolution of friction at depth in snow under varying overburden pressure (McClung and Schaerer (1993), Nakamura et al. (2010) etc.) and is consistent with expectations.

Colbeck (1988) (commenting on an observation by Perla and Glenne (1981)) says that for pressures typically exerted by skiers $(1-5 \mathrm{kPa})$ there appears to be negligible variation in friction with increased load, a consequence of the true contact area being proportional to the load; this may be the case on the surface of natural snow. However, during penetration it is assumed that processed, rearranged, compacted and densified 


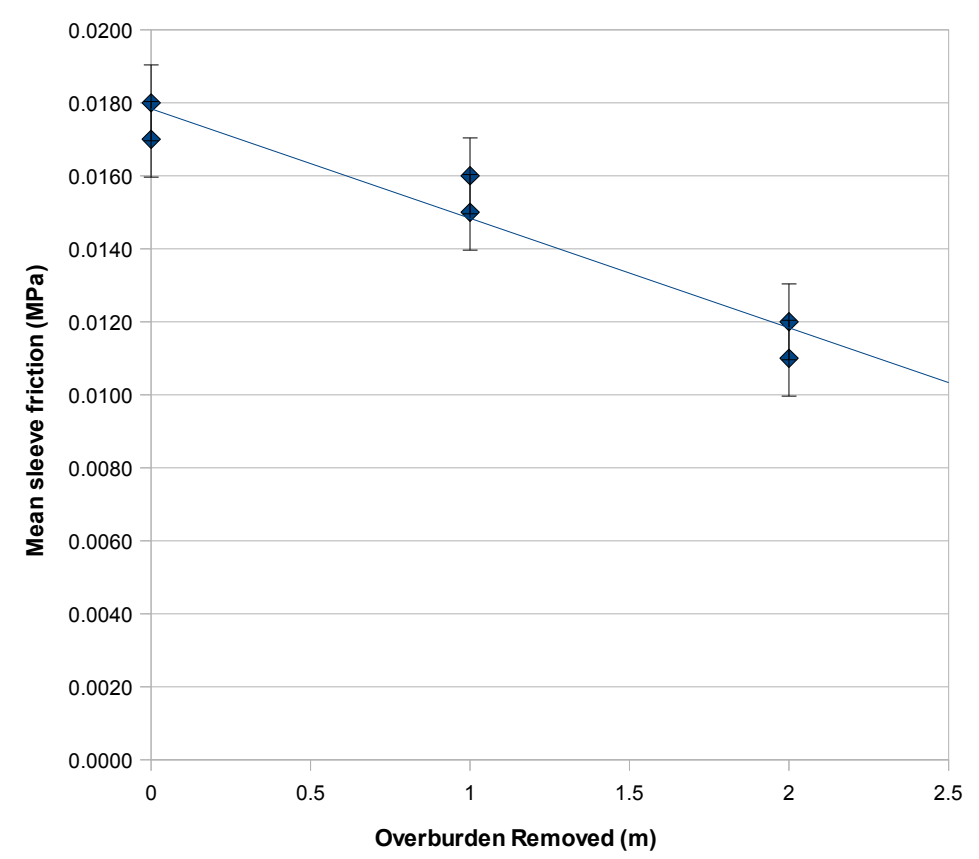

Figure 5.30: Significant variation of mean sleeve friction with amount of overburden removed.

snow, beside the friction sleeve, is of a largely uniform nature such that any increase in normal pressure will result in an increase in friction upon the sleeve. Also, pressure upon the friction sleeve is estimated to be an order of magnitude greater than these values considered by Colbeck (1988).

Although these data were probably affected to some extent by the testing method, some decrease in observed sleeve friction owing to decreased overburden pressure is apparent.

\subsection{Pressure melting}

Both Federolf et al. (2004) and Szabo and Schneebeli (2007) note the rapid, almost immediate sintering of snow grains immediately after brittle fracture, and some level of pressure melting and refreezing of fractured bonds was expected as the cone was driven into the snow, possibly sufficient to 'freeze-in' the cone and rods. However, no level of static friction was evident upon retrieval, nor was any free water observed, perhaps suggesting that temperature and snow-moisture conditions were sufficiently cold and dry to limit identifiable pressure-melting.

Floyer (2008) cautions against stopping during penetrometer pushes, because fractured snow could re-bond, resulting in force spikes (not related to actual snow hardness) upon recommencement of a push. Clearly, melting and resintering was possible, yet none was visibly or aurally evident whilst conducting CPT, nor does examination of CPT data suggest any freezing-in occurred. During Test 52 where the cone was left 
stationary insitu for almost one hour during a dissipation test, no 'spike' in resistance was observed upon resumption of the test. This may be because of the limited data sampling of the equipment used, or may be evidence of a lack of pressure melting.

A simple quantitative assessment of this phenomenon can be obtained by again considering melting point depression. Cone tip resistance values were typically $<10$ MPa hence at snow temperatures of $\sim-10^{\circ} \mathrm{C}$ it is extremely improbable that melting occurred. This is substantiated by Barnes et al. (1971) who found that when brass slid on ice (at maximum applied pressure of $\sim 1 \mathrm{MPa}$ ) no melting occurred at speeds less than $100 \mathrm{~mm} \mathrm{~s}^{-1}$ at a temperature of $-12^{\circ} \mathrm{C}$. The increase in pressure required to generate melting during penetration does not appear likely within the conditions of testing discussed herein.

\subsection{Dissipation Tests}

In standard CPT within saturated soils dissipation tests are routinely carried-out to assess the dissipation of any excess pore-pressure over time (Lunne et al., 1997). Although no free water and thus no pore pressure (due to liquid) will be evident in the snow under test at Halley $\mathrm{V}$, it was deduced that the dissipation test was a potentially valuable tool in assessing the change in tip resistance over time upon stopping penetration. A number of dissipation tests were therefore conducted at various depths and for various periods of time in order to investigate this otherwise un-examined phenomena in snow. Figure 5.31 displays the result of four of these tests.

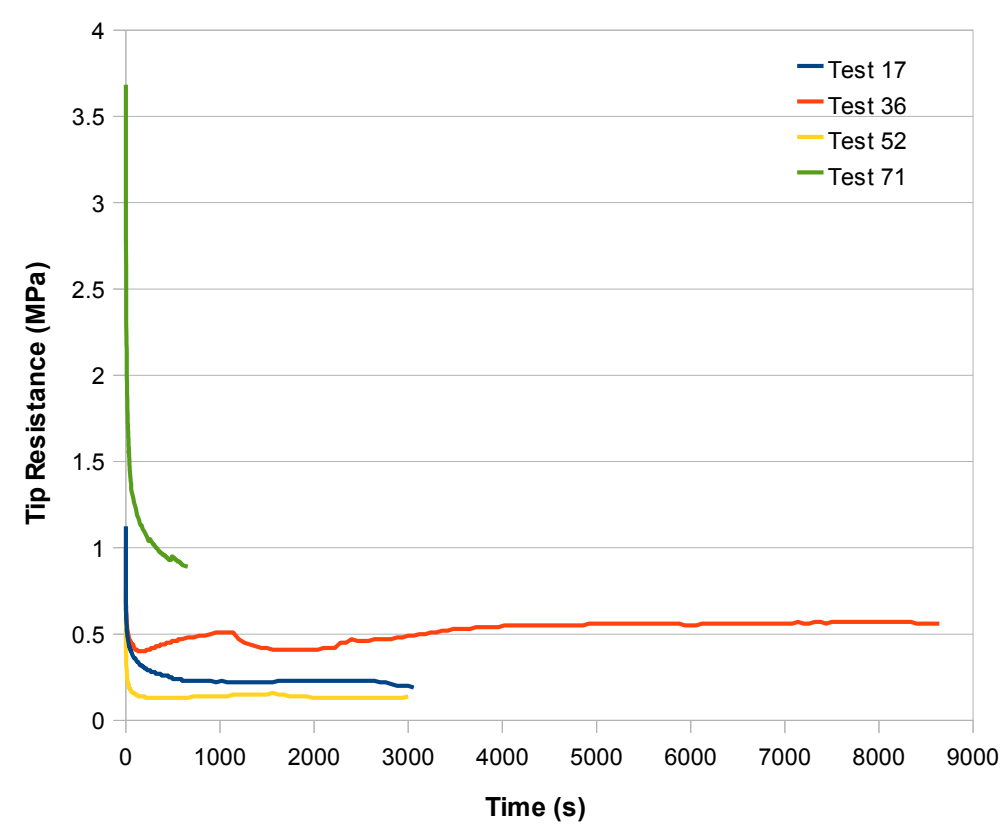

Figure 5.31: Cone tip resistance decreases from the time $\mathrm{t}=0$ when penetration is stopped.

A measured decrease in tip resistance is observed for all tests and Table 5.2 outlines 
relevant parameters:

Table 5.2: Dissipation Test Data

$\begin{array}{llllllll}\text { Test } & \begin{array}{l}\text { Test } \\ \text { Depth } \\ (\mathbf{m})\end{array} & \begin{array}{l}\text { Test } \\ \text { Length } \\ (\mathbf{s})\end{array} & \begin{array}{l}\text { Initial tip } \\ \text { resistance } \\ (\mathbf{M P a})\end{array} & \begin{array}{l}\text { Reduction } \\ \text { in } \\ \text { tance }\end{array} & \begin{array}{l}\text { Estimated } \\ \text { insitu ver- } \\ \text { tical stress } \\ (\mathbf{k P a})\end{array} \\ 17 & 2.00 & 3060 & 1.13 & 83 & 8.8 \\ 36 & 1.00 & 8640 & 0.995 & 44 & 4.4 \\ 52 & 0.97 & 3000 & 0.76 & 82 & 4.3 \\ 71 & 2.92 & 660 & 3.68 & 86 & 12.9\end{array}$

Note that the reduction in tip resistance for Test 71 is estimated, based on a powerlaw trend line, similar to the curve that provides best-fit to the three other curves.

Three of the tests show a decrease in tip resistance over time of between 82 and $86 \%$. The remaining test (Test 36) shows an initial decrease in tip resistance of $\sim 60 \%$ before resistance rises. This test was the longest test, and perhaps all tests may have exhibited such behaviour over an extended time. Such testing is not known to have been previously conducted in snow, hence no literature is drawn upon in the discussion below. The tip resistance measurements are not observed to dissipate towards the estimated in situ vertical stress ( $5-15 \mathrm{kPa}$ for snow of density $450 \mathrm{~kg} \mathrm{~m}^{-3}$ ), residual resistances are not related to rod length/weight, nor do they appear to exhibit any other obvious trend. It appears that a process of relaxation is taking place. Three (amongst possibly other) factors are:

1. elastic relaxation in the rods,

2. relaxation in the pushing equipment, and

3. some deformation mechanism within the snow.

The rods are under compression during a test, so any cessation of pushing should allow them to relax (expand in length) resulting in an increase in measured tip resistance. Such an increase is not observed. This is because the steel rods (with a Young's Modulus of $210 \mathrm{GPa}$ ) would only increase in length by $\sim 0.01$ to $\sim 0.05 \mathrm{~mm}$ once pushing has stopped, and such a small displacement will easily be accommodated within relaxation of surface pushing equipment. Upon cessation of pushing it is likely that the snow at the head of the cone will possibly relax elastically to a very small amount (\% of a $\mathrm{mm}$ ) and then sinter. However, no significant (or measurable) compaction or expansion of the snow is expected during this process.

There is significant 'slop' in the pushing equipment within UK11 and it is proposed that most of the measured relaxation of tip resistance is owing to strains within this system being released over time, resulting in a diminished (although still greater than insitu stress) tip resistance over time. It is also not known upon halting a CPT whether 
the release of the hydraulic lever may cause a minor and temporary vacuum within the hydraulic system, potentially partially withdrawing the rod/cone string, thus reducing the measured tip resistance; no pressure measure existed with which to examine this process.

Although it was envisaged that the conduct of the dissipation test may provide valuable insight into the post-penetrative behaviour of the fractured snow at the head of the cone, it is likely that most if not all of the behaviour observed is because of relaxation in the pushing equipment, which was observed to contain many millimetres of slack during use. It is not known why a subsequent increase in tip resistance was observed in Test 36 .

\subsection{Cyclic Testing}

Cyclic penetrometer testing is routinely performed in clays, particularly soft sub-sea sediments, as a means of assessing the remoulded shear strength of the soil. Shear strength will generally deteriorate with each cycle, eventually approaching a lower 'remoulded' value after between 10 to 30 cycles. It is typically conducted using a larger diameter ball-penetrometer, rather than a cone.

Discussion with R. Santos, Geotechnical Advisor at Gardline Geosciences Limited, January 2010 suggested that such testing may be worth pursuing. However, my limited experience in the conduct of such testing and the difficulty in obtaining sufficient interpretable data meant that such testing was terminated after one attempt. Further effort in assessing the resistance change of snow under repetitive loading via CPT remains of interest. It is speculated that depending on the temperature of the snow and the heat generated through such loading, that progressive deterioration of the material and a complete loss of cohesion known as fluidization (Abele, 1970) might occur, else possibly regelation, where some melting under pressure, then refreezing of the material may occur.

\subsection{Interpretation via Standard Soil Charts}

Similarities between snow and other geomaterials have previously been discussed within this work therefore qualitative and quantitative comparison of snow CPT data with standard CPT soil interpretation charts is of interest. Numerous authors have produced such charts (Robertson (1990), Douglas and Olsen (1981), Ramsey (2002) etc.) many of which are collected in Lunne et al. (1997). An example of a soil classification chart prepared by Robertson et al. (1995) is presented in Figure 5.32:

In the context of such charts, what are the broad characteristics of the CPT data obtained at Halley? The CPT data typically exhibits:

1. low cone resistance $(<10 \mathrm{MPa})$, and 


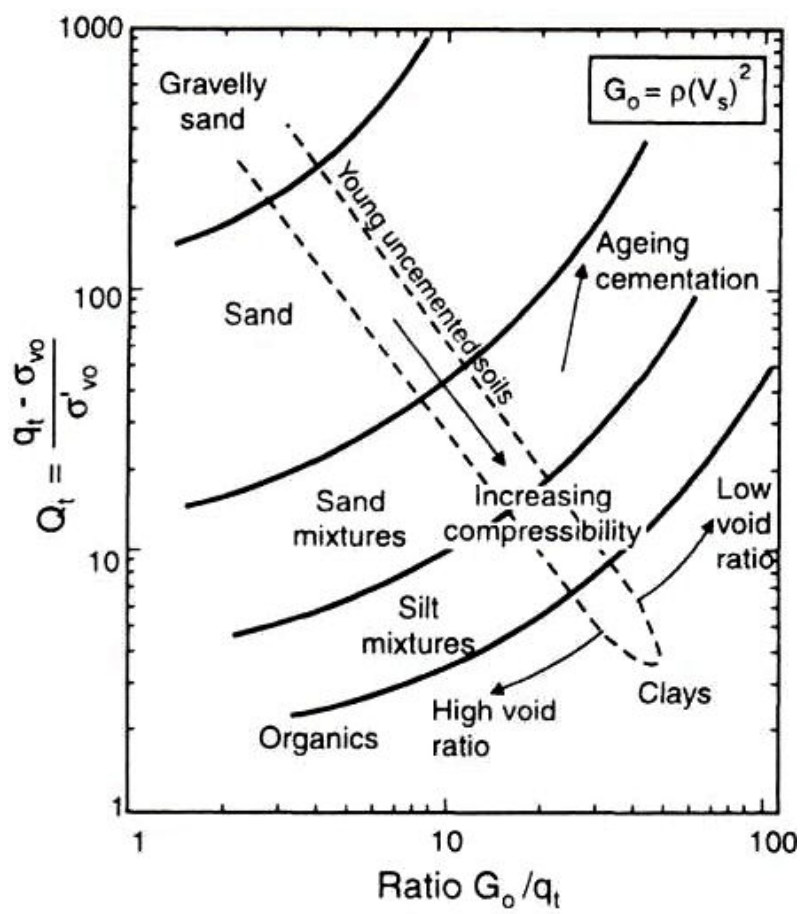

Figure 5.32: Soil classification chart based on normalised cone resistance $\left(q_{t}\right)$ and small strain shear modulus $\left(G_{o}\right)$ (see Glossary) (from Lunne et al. (1997)).

2. friction ratio $\leq 1 \%$.

Such quantitative descriptors are consistent with highly compressible (such as carbonate or calcareous) sands (Lunne et al., 1997), and when applied to various CPT soil interpretation charts presented within Lunne et al. (1997) and Robertson and Cabal (2010) the following descriptors are found:

1. sensitive fine grained soils through to sand, where tip resistance increases with relative density,

2. sensitive, fine grained soil, and

3. extremely compressible soil.

Although a very different geomaterial, classification of snow via existing CPT soil interpretation charts yields descriptors that appropriately describe some of snow's unique characteristics.

\subsubsection{Comparison with cemented sands}

Comparison with the classification system proposed by King et al. (1980) for carbonate sediments suggests that similarities exist between snow and coarse, weakly to firmly cemented carbonate sands. For such sands, Beringen et al. (1982) empirically derived an $N_{K}$ (cone factor) value of between 15 and 20, relating cone tip resistance to shear strength, $N_{K}=q_{c} / s_{u}$. If a similar cone factor is applied to a typical cone tip resistance 
of say $1 \mathrm{MPa}$, then a shear strength of $\sim 50-70 \mathrm{kPa}$ is empirically derived, consistent with the range of expected shear strength of bonded snow $(\sim 60 \mathrm{kPa}-200 \mathrm{kPa})$ for density $\sim 450 \mathrm{~kg} \mathrm{~m}^{-3}$, at a temperature $-3^{\mathrm{O}} \mathrm{C}$ to $-10^{\circ} \mathrm{C}$ (Mellor, 1975). If Test 71 specifically is examined, a steady tip resistance of $\sim 1.8 \mathrm{MPa}$ from $\sim 1.25 \mathrm{~m}$ (assumed representative) equates to a shear strength of $\sim 90-120 \mathrm{kPa}$. This layer (from snow pit \#2 data) was of density $459 \mathrm{~kg} \mathrm{~m}^{-3}$, with 'pencil' hardness and with grain size $0.7 \mathrm{~mm}$. This shear strength derived empirically from tip resistance locates centrally within the historical data presented in Mellor (1975) (60 - $250 \mathrm{kPa}$ ). These comparisons serve to suggest order of magnitude similarities between the cone factor derived for cemented carbonate sands and representative values for snow, but are not sufficient to suggest that the $N_{K}$ value for snow is also between 15 to 20. It is likely of similar magnitude, and further physical reasoning on the establishment of such a cone factor for snow occurs in Chapter 7.

This comparison suggests similarities in mechanical behaviour between the two materials, and such similarity is not unexpected as both media consist of bonded granular material which are expected to fracture upon penetration resulting in a frictional granular medium. Such similarity is endorsed via Johnson's 2003 work, A Statistical Micromechanical Theory of Cone Penetration in Granular Materials (Johnson, 2003) in which snow is considered as the primary granular material under question. Further comparison between the behaviour of dry bonded polar snow and cemented sands is of interest, especially noting the substantial work that has occurred into the engineering use of such material in offshore environments (see for example Dean (2010)).

\subsection{Snow microstructure from CPT}

Before concluding this chapter a brief mention is made of the possibility of detecting snow microstructure from CPT data. Changes in resistance measured by the cone may be because of changes in density or changes in microstructure; different stress is needed to compact snow of varying initial density and increased stress is needed to fracture more, or more-developed bonds between ice grains. Tip resistance is seen to vary with density (on a layer) scale, and generally microstructure and density will go hand in hand, so that an increase in microstructure (i.e. increased number and/or thickness of bonding) will generally result in increased density. However, an increase in density (more mass per volume) does not necessarily mean an increase in microstructure (an increase in bonding).

In homogeneous snow it might be expected that sleeve friction would be correlated with tip resistance, and even in the heterogeneous data presented in this work thus far, this appears the case. Therefore the ratio between these two values might be expected to be approximately constant; this value has been previously described and is known as the friction ratio, $R_{f}=f_{s} / q_{c} \times 100 \%$. 
As the level of bonding increases, this ratio should start to deviate. Sleeve friction readings should remain similar, as the fractured and compacted snow forming the hole annulus is indifferent to the amount of initial bonding whereas tip resistance will increase, owing to the need to now fracture more bonds. Lee and Huang (2010) in $\mathrm{CPT}$ on cemented sand found that the increase in tip resistance between unbonded and bonded material can be up to 4 to 5 times. Friction ratio should therefore decrease as the amount of bonding increases, so can this variation in friction ratio suggest variation in snow microstructure?

Figure 5.33 shows that friction value ratios for Test 9 vary erratically with depth, and this is not unexpected because of the many factors that affect tip resistance, sleeve friction and thus friction ratio.

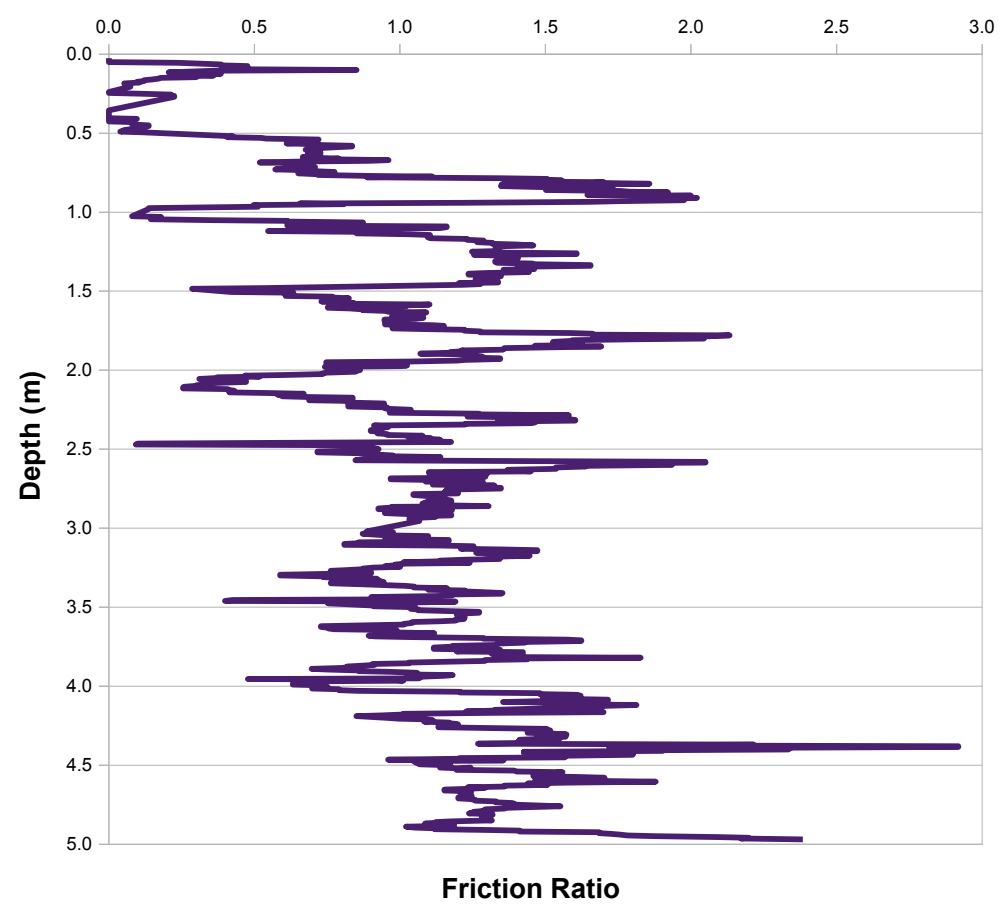

Figure 5.33: Data from Test 9 shows that friction ratio varies erratically with depth because of the many factors that affect tip resistance, sleeve friction and thus friction ratio.

However, if these data are presented as a scatterplot, useful information may be extracted. Figure 5.34 shows the variation in tip resistance versus normalised sleeve friction for Test 9 as a scatter plot, with linear trend line.

What this figure shows is that for each sleeve friction value (y-axis) there is a range of tip resistance values (x-axis). Whilst some of this variation may be because of changes in density, any variation at the same density may be indicative of variations in microstructure; higher tip resistance for the same sleeve friction suggesting a morebonded layer.

This supposed phenomenon is difficult to illustrate. However, examination of snow pit \# 1 data suggests that some layers were encountered with similar density but different microstructure (as determined by observation, grain size and hardness). Compari- 


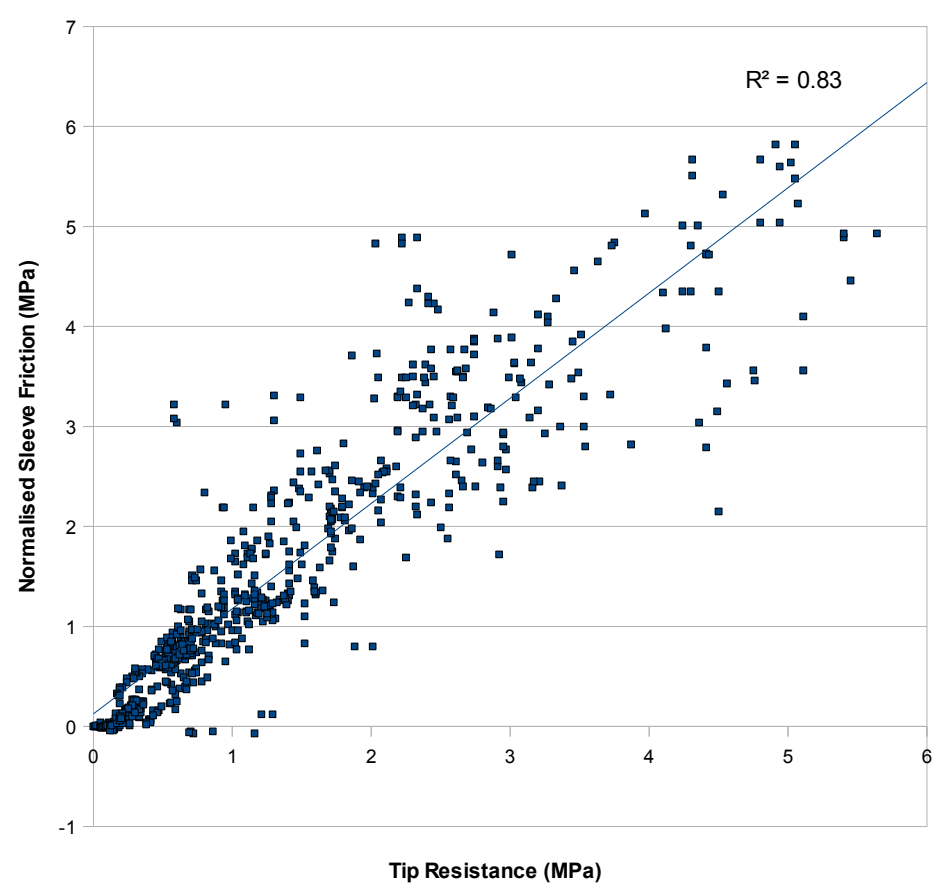

Figure 5.34: Scatter plot of tip resistance and sleeve friction. Different tip resistance at the same sleeve friction value may suggest snow microstructure.

son between these layers may be expected to reveal similar sleeve friction values, but different tip resistance values, evidenced by a large difference in friction ratio. Table 5.3 compares these limited data.

Table 5.3: Friction Ratio variation between similar layers.

\begin{tabular}{|c|c|c|c|c|c|}
\hline Layer & $\begin{array}{l}\text { Depth } \\
(\mathrm{cm})\end{array}$ & $\begin{array}{l}\text { Density } \\
\left(\mathrm{kg} \mathrm{m}^{-3}\right)\end{array}$ & Hardness & $\begin{array}{l}\text { Grain } \\
(\mathrm{mm})\end{array}$ & $\begin{array}{l}\text { Average } \\
\text { Friction } \\
\text { Ratio }\end{array}$ \\
\hline 2 & 10 to 57 & 420 & Finger & 0.8 & 0.25 \\
\hline 20 & $\begin{array}{ll}248 & \text { to } \\
252 & \end{array}$ & 416 & Finger/Pencil & 1.3 & 0.9 \\
\hline
\end{tabular}

Although Layer 2 is slightly harder (via the subjective hand-hardness test) it has the smallest grain size, suggesting greater number of bonds per volume of snow. Layer 2 also has the smallest friction ratio. This is consistent with expectations: friction ratio should decrease as the amount of bonding increases. This observation suggests that a correlation between friction ratio and microstructure may be warranted. However, owing to the complicated nature of the field data, further extensive laboratory testing in pre-defined snow is recommended in order to further investigate this relationship. Such findings further highlight that attempting to categorise snow by any one variable, density, hardness, grain size etc. will always be problematic and that any snow layer will often provide a unique combination of these parameters. 


\subsection{Summary}

Discussion in this and the previous chapter focused on the empirical observation and comparison of penetrative data in polar snow. This chapter in particular has examined in detail many facets pertinent to the interpretation of CPT in polar snow. Variation of CPT parameters in snow appears to be dependant upon the nature of the snow, the size and shape of the penetrometer, and the range of penetration and thus strain rates utilised. All of these factors should be considered in interpreting penetrative tip resistance and sleeve friction data. The next chapter takes a step back from penetrative testing and examines additional subsurface investigations that were carried out at Halley. Data from this testing are valuable in further assessing the application of CPT in later chapters. 


\section{Chapter 6}

\section{Results \& Analysis (C) - Supplementary subsurface investigations}

This chapter presents results and analysis of the supplementary subsurface testing that was carried out to assist in the interpretation and application of the CPT conducted in Antarctica. Chapter 7 will then incorporate this analysis.

\subsection{Density Assessment}

Two snow pits were dug for the purpose of assessing snow stratigraphy and layer density, and for comparison with both cone penetration tests and GPR surveys. The location of these pits is shown in Figure 3.5. Data for pit \#1 including stratigraphy, density, grain size, hardness and snow type is presented in Figure 6.1, generated using SnowPilot (Chabot and Kahrl, 2009). SnowPilot's data fields are consistent with the guidelines published within "Snow, Weather, and Avalanches: Observational Guidelines for Avalanche Programs in the United States" (American Avalanche Association, 2004). Data for Pit \#2, which was located $~ 1.5$ m east are presented in Figure 6.2.

Initial comparison between the two pits suggests similarity in layering, and an obvious seasonal trend, although a depth displacement of perhaps 50 to $60 \mathrm{~cm}$ is apparent between pits. Grain sizes found within both pits varied little, ranging from 0.4 to $1.2 \mathrm{~mm}$, with an average size of $\sim 0.8 \mathrm{~mm}$, perhaps somewhat larger than the mean Antarctic grain size of $\sim 0.5 \mathrm{~mm}$ (down to $5 \mathrm{~m}$ ) assessed by Rick and Albert (2004), probably owing to the warmer temperatures generally experienced at the coastal Halley station.

Although the two snow pits were located only $1.5 \mathrm{~m}$ apart, differences in stratigraphy are observed. Pit \#2 commenced at a surface level some $15 \mathrm{~cm}$ below Pit \#1, however, significant snow $(\sim 30 \mathrm{~cm})$ had fallen between construction of the first and second pits, hence stratigraphy noted within Pit \#2 may be higher relative to Pit \#1. Conversely, some compaction of the snow at the \#2 location probably occurred during initial work at Pit \#1 (including passes by the tractor) hence differences in 
CHAPTER 6. RESULTS \& ANALYSIS (C) - SUPPLEMENTARY SUBSURFACE INVESTIGATIONS
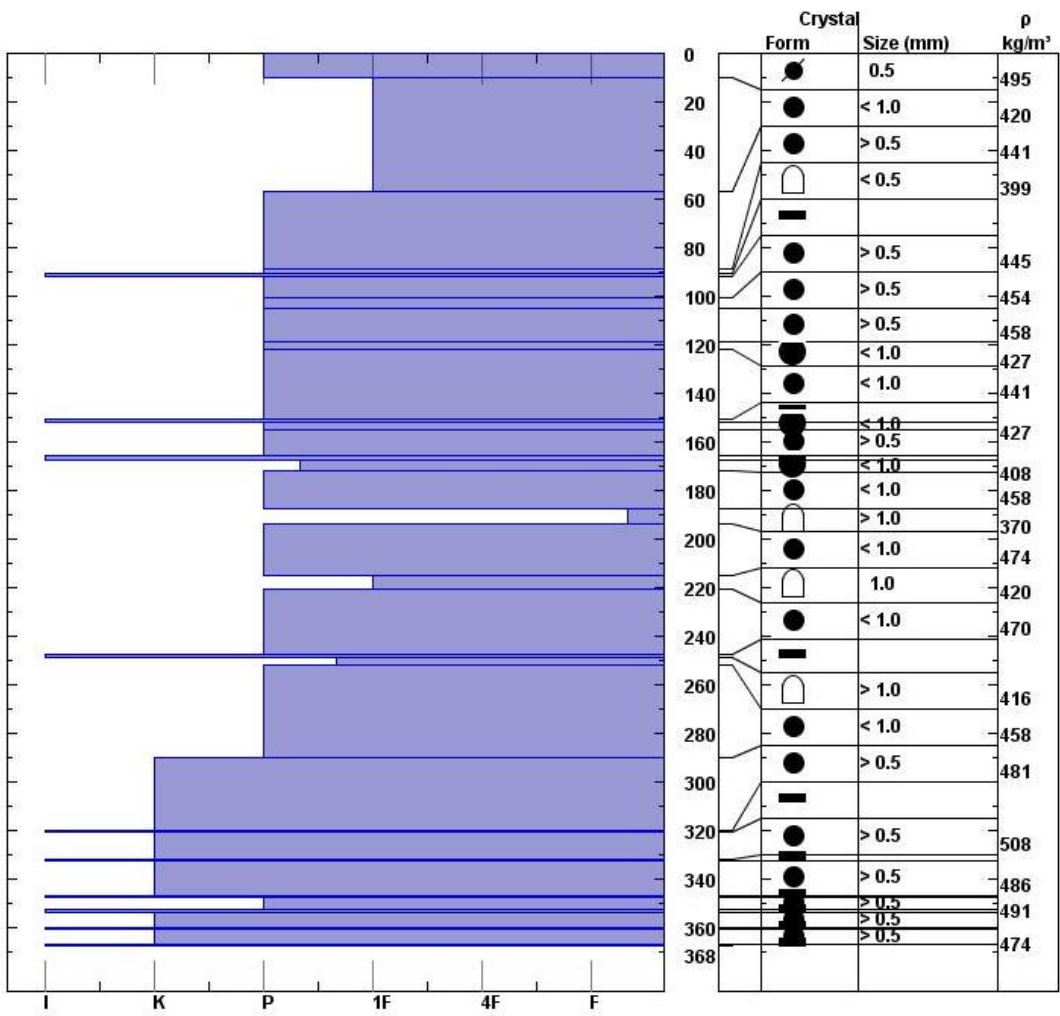

Figure 6.1: Snow Pit \#1 data showing layer hardness (Ice, Knife, Pencil, 1 Finger, 4 Fingers, Fist), crystal type, grain size and density.
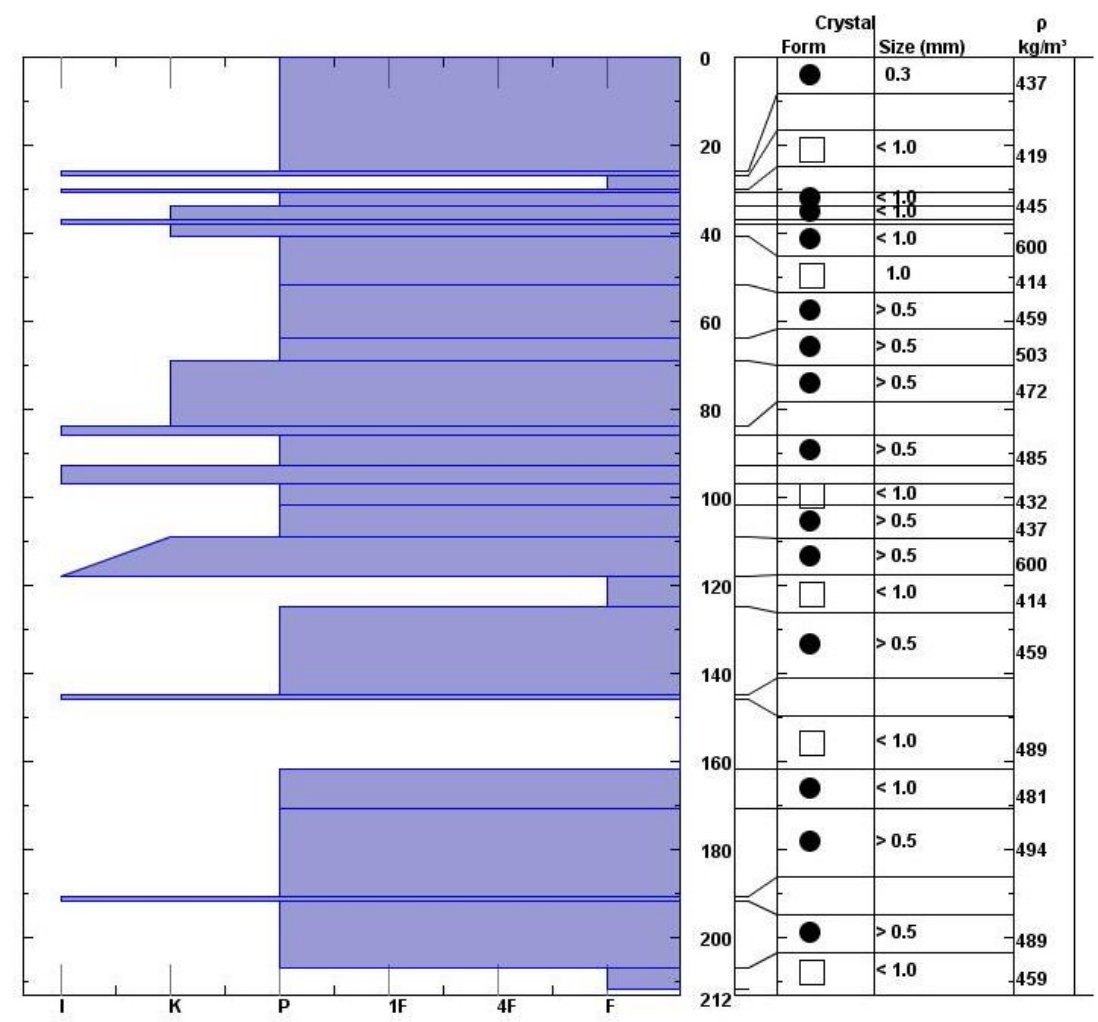

Figure 6.2: Snow Pit \# 2 data showing layer hardness (Ice, Knife, Pencil, 1 Finger, 4 Fingers, Fist), crystal type, grain size and density. 
the upper layers because of both accumulation and compaction is likely, complicating comparison. Direct comparison of stratigraphy between pits is not easily achievable; if anything, alignment between pits appears possible if a depth of some $80 \mathrm{~cm}$ is added to Pit \#2 data. Further comparison is not undertaken, although it does suggest (unexpected) variation at small spatial scale.

Hardness observed within the pits appears very consistent, with the majority of layers displaying 'Pencil' hardness between occasional ice layers. Weak layers consisting of large loosely bonded grains were evident within each pit, typically found beneath an ice layer. This form of layering is consistent with that described by Goodwin (1991) in eastern Wilkes Land, Antarctica where depth hoar (formed from surface hoar in mid-late summer) was typically found beneath a hard spatially-continuous surface wind-glazed ice crust that formed in autumn. Similar 'blue' ice-bands existing "in and below" the summer coarse-grained layer were also identified by MacDowall (1964) in his glaciological investigations at Halley Bay in 1956. The equivalent force value of these hardness data and comparison with CPT resistance values are discussed in the next chapter.

\subsection{Confined compressive strength testing}

In Section 2.2.1 it was noted that resistance to penetration is equivalent to strength in compression. Thirty-one confined-compression tests (or plate insertion tests) were conducted on samples with density ranging from 420 to $508 \mathrm{~kg} \mathrm{~m}^{-3}$, in an attempt to obtain some measure of snow strength within snow pit \#1, for comparison with CPT data.

Figure 6.3 presents the raw data of the thirty-one tests in a plot of resistance (MPa) versus time (s). This plot includes the withdrawal of the plate upon completion of the test. Figure 6.4 is an adaption of Abele's load-sinkage diagram (Abele, 1970) suggesting possible relations between applied pressure and plate sinkage in snow. (Note the axes in Figure 6.3 are rotated by $90^{\circ}$ compared to comparable axes in Figure 6.4).

The variation in stress with time (depth) from this testing, at a high penetration rate $\left(38 \mathrm{~mm} \mathrm{~s}^{-1}\right)$ is expected to be of a form consistent with the addition of Abele's 'collapse' curve with the 'compaction' curve, possibly of a form presented in Figure 6.5, and some results from Figure 6.3 appear to comply with this supposition. Discussion of this fracturing then compacting process occurred in Chapter 2.

In some cases a preliminary (collapse) peak was not identifiable. This may be because such a collapse peak did not exist, or because the nature of the data-acquisition system (Golog software used in dissipation test mode, sampling at only $1 \mathrm{~Hz}$ ) meant that such a peak was not recorded. Table 6.1 therefore summarises the confined compressive strength versus density data in which an initial (collapse) peak was identifiable, and Figure 6.6 presents this averaged strength data with density. 


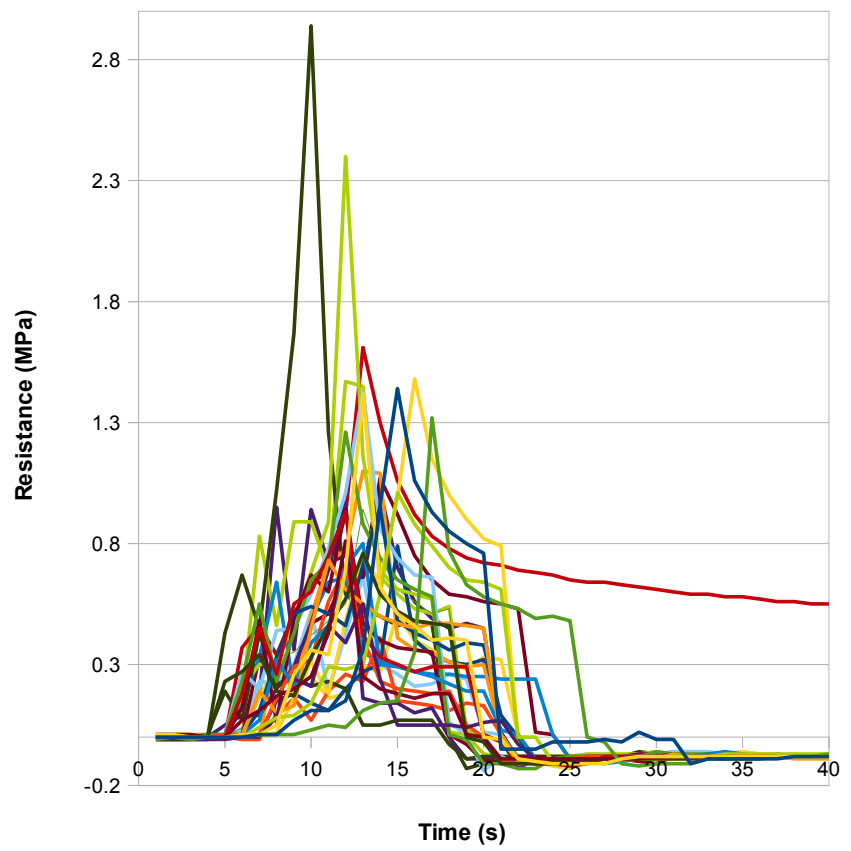

Figure 6.3: Unprocessed confined compression strength testing data typically showing two peaks, one owing to initial failure and a second as the base of the test cylinder is approached; a reduction in stress is then seen as the plate is withdrawn.

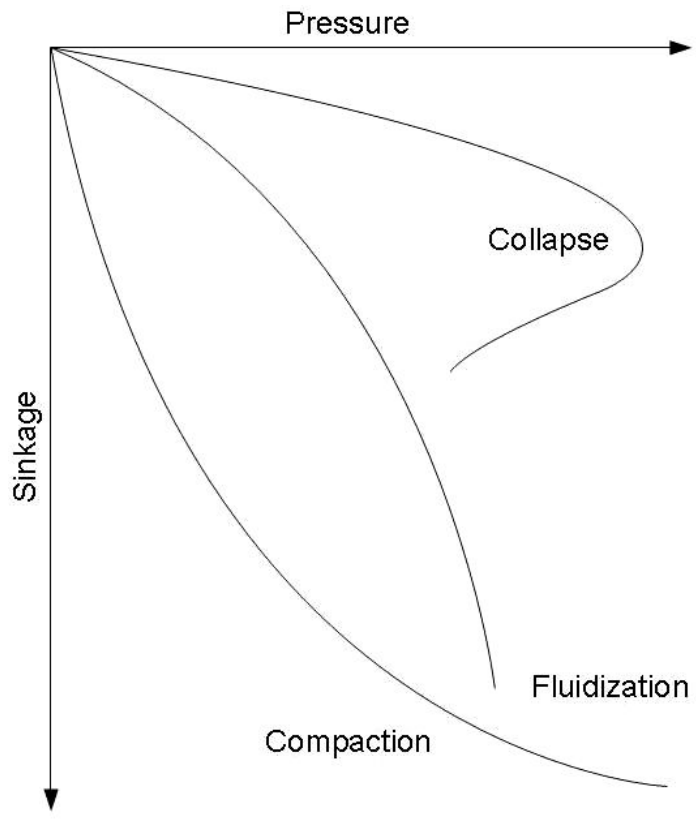

Figure 6.4: Adaption of Abele's (1970) load-sinkage relation figure.

A large amount of scatter is evident (as is normal within the strength testing of snow, at least when plotted against density (see Mellor (1975), Shapiro et al. (1997) etc.) and a linear trend line suggests a poor correlation. Although the presentation of the resistance data in this manner (versus density) is commensurate with standard and historical practice, it is not necessarily the preferred index by which to characterise snow mechanical properties. These compressive strength data are now compared with 


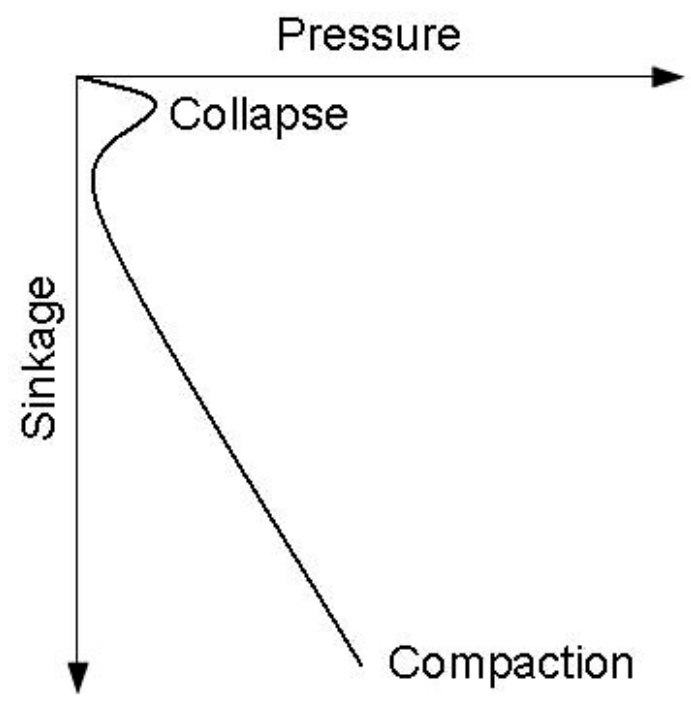

Figure 6.5: Possible form of expected pressure-sinkage relationship shows initial collapse followed by pressure increase as compaction continues.

Table 6.1: Resistance under confined compression versus density.

$\begin{array}{cr}\text { Density }\left(\mathrm{kg} \mathrm{m}^{-3}\right) & \text { Resistance } \\ 420 & 0.36 \\ 458 & 0.34 \\ 458 & 0.29 \\ 458 & 0.24 \\ 441 & 0.19 \\ 441 & 0.45 \\ 441 & 0.32 \\ 478 & 0.54 \\ 478 & 0.74 \\ 458 & 0.55 \\ 458 & 0.48 \\ 458 & 0.27 \\ 470 & 0.33 \\ 470 & 0.46 \\ 470 & 0.64\end{array}$

published strength and density data.

Abele (1990) in his work on the deformation of snow under rigid plates found a relationship between unconfined compressive strength $\left(\sigma_{u}\right)$ as a function of density $(\rho)$ (for the density range 0.28 to $0.59 \mathrm{~g} \mathrm{~cm}^{-3}$ ) of $\log \sigma_{u}=\mathrm{a} \rho+\mathrm{b}$. Fitting of this function to my data using Abele's values of $a=0.01$ and $b=-2.74$ provides a poor fit, with $R^{2}$ of only 0.24 , because of the large scatter exhibited within the results.

If, however, only the maximum values for each density are retained then a much better fit of $\mathrm{R}^{2}=0.99$ is obtained, with an almost identical gradient. This fit generates values of $\mathrm{a}=0.01$ and $\mathrm{b}=-2.67$, almost identical to Abele's suggesting that this trend may actually be valid. My data (red squares) is shown superimposed over Abele's 


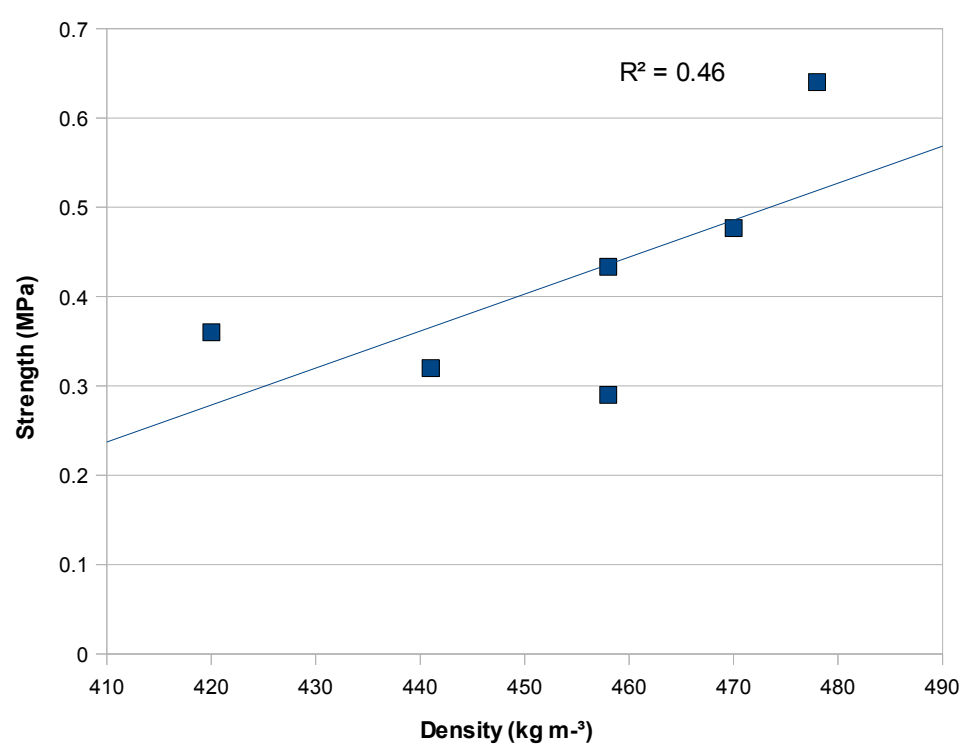

Figure 6.6: Average strength versus density (from selected confined compression tests) for snow samples taken from within snow pit \#1.

original data in Figure 6.7.

The measured compressive strength values of between $\sim 0.2$ and $0.75 \mathrm{MPa}$ for snow densities 420 to $480 \mathrm{~kg} \mathrm{~m}^{-3}$ (see Figure 6.6) are consistent with those reported by Kinosita (1967) (0.2 MPa to $0.8 \mathrm{MPa}$ ) in testing under uniaxial strain at a strain rate $\sim 10^{-4} \mathrm{~s}^{-1}$ as published by Mellor (1975).

Although limitations in instrumentation and snow-sample extraction hampered the consistency of this plate-testing, values of compressive strength obtained using the CPT equipment appear broadly consistent with published data. Further comparison with resistance determined via CPT will occur in the next chapter, and discussion pertaining to bearing capacity ensues in Chapter 8. Additional first-hand strength data was desirable to compare with CPT data, thus the adoption of additional shear strength testing, described next.

\subsection{Strength Testing (Shear Frame)}

Numerous shear strength tests were conducted using various frame sizes and forms. The data presented here are from only two sets of tests conducted on the 11th and 12th Feb 2010; other tests were of a trial nature and did not generate useful data. Testing on the 11th was on a homogeneous surface layer adjacent to snow pit \#1, whereas testing on the 12 th was on heterogeneous samples within the Halley V vehicle parking area (see Figure 3.5). These tests are summarised in Tables 6.2 and 6.3, whilst Figure 6.8 presents data from Table 6.3 in graphical form; a linear trend-line is also presented.

Although the shear-strength testing proved problematic (partial fracturing of blocks, 


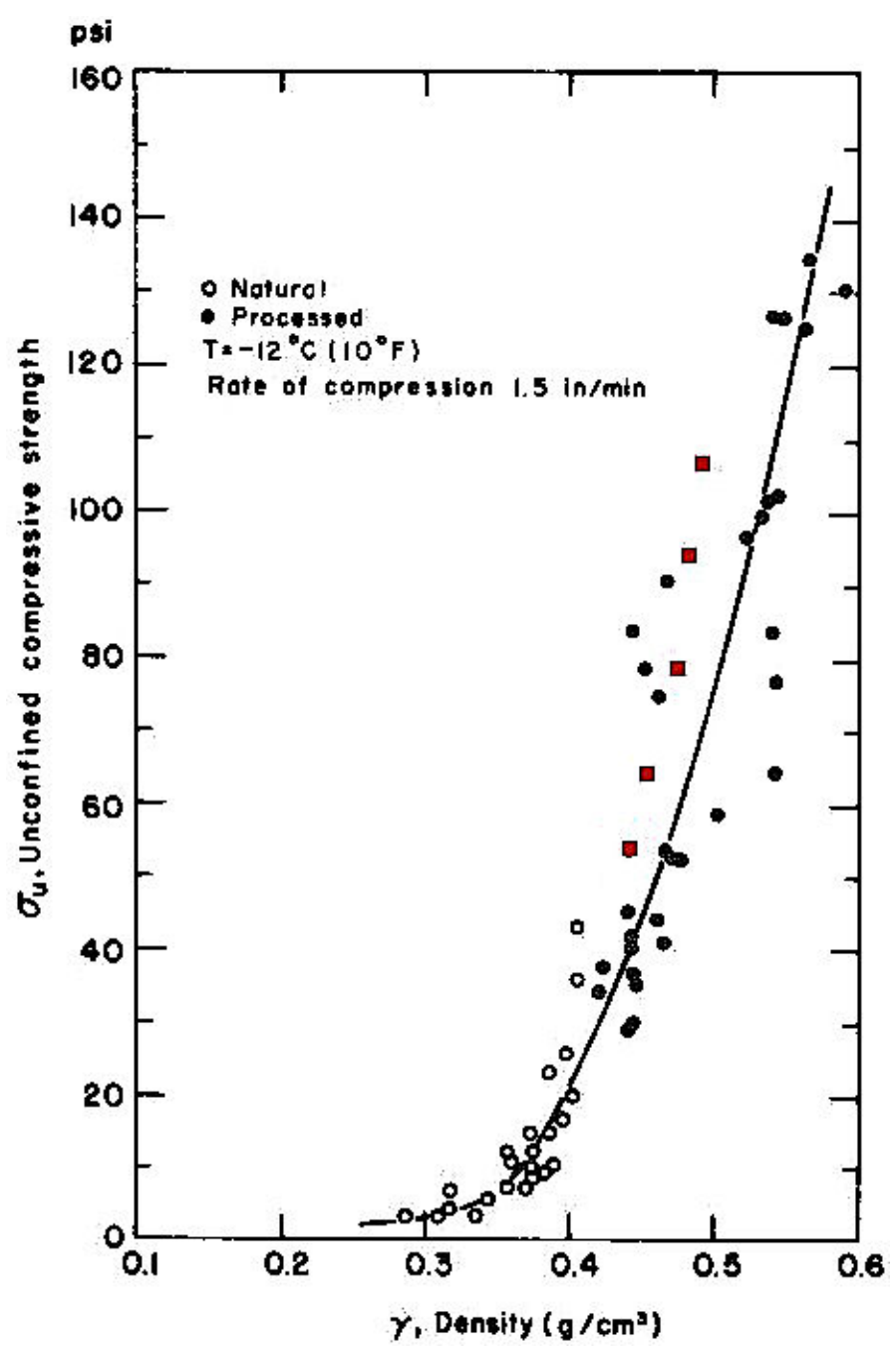

Figure 6.7: Halley compressive strength data (red squares) is consistent with compressive strength vs density values from Abele (1990).

Table 6.2: Shear strength $(\mathrm{kPa})$ versus density - 11th Feb 2010.

\begin{tabular}{|c|c|c|c|c|}
\hline Test & Layer & 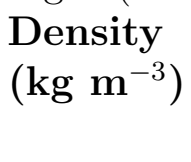 & $\begin{array}{l}\text { Shear } \\
\text { Strength } \\
(\mathrm{kPa})\end{array}$ & Remarks \\
\hline 1 & $\begin{array}{l}\text { surface layer } \\
\text { (top } 5 \mathrm{~cm})\end{array}$ & 390 & 12.5 & $\begin{array}{l}\text { Fracture on insertion } \\
\& \text { 'skipping'. }\end{array}$ \\
\hline 2 & surface layer & 390 & 15 & Fracture on insertion. \\
\hline 3 & surface layer & 390 & 12.5 & \\
\hline 4 & surface layer & 390 & 12.5 & \\
\hline 5 & surface layer & 390 & 5 & Only 1/3 mobilised. \\
\hline 6 & surface layer & 390 & 2.5 & No shear just rotation. \\
\hline 7 & surface layer & 390 & 2.5 & $\begin{array}{l}\text { Fracture upon inser- } \\
\text { tion. }\end{array}$ \\
\hline 8 & surface layer & 390 & 17.5 & Good test. \\
\hline 9 & surface layer & 390 & 15 & 'Skipped'. \\
\hline 10 & surface layer & 390 & 2.5 & $\begin{array}{l}\text { 'Skipping' \& fracture } \\
\text { upon insertion. }\end{array}$ \\
\hline
\end{tabular}


Table 6.3: Shear strength (kPa) versus density - 12th Feb 2010.

\begin{tabular}{|c|c|c|c|c|}
\hline Test & Layer & $\begin{array}{l}\text { Density } \\
\left(\mathrm{kg} \mathrm{m} \mathbf{m}^{-3}\right)\end{array}$ & $\begin{array}{l}\text { Shear } \\
\text { Strength } \\
(\mathrm{kPa})\end{array}$ & Remarks \\
\hline 1 & $\begin{array}{l}\text { surface layer } \\
(\text { top } 10 \mathrm{~cm})\end{array}$ & 500 & 17.5 & \\
\hline 2 & surface layer & 600 & 17.5 & $\begin{array}{l}\text { Partially } \\
\text { fractured. }\end{array}$ \\
\hline 3 & surface layer & 600 & 20 & $\begin{array}{l}\text { Partially } \quad \text { pre- } \\
\text { fractured. }\end{array}$ \\
\hline 4 & surface layer & 465 & 22.5 & \\
\hline 5 & surface layer & 465 & 27.5 & \\
\hline 6 & surface layer & 600 & 30 & $\begin{array}{l}\text { Partially } \quad \text { pre- } \\
\text { fractured. }\end{array}$ \\
\hline 7 & $\begin{array}{l}>10 \mathrm{~cm} \text { depth } \\
\text { with ice layers }\end{array}$ & 500 & 37.5 & $\begin{array}{l}\text { 'Skipped' - only one } \\
\text { fragment sheared. }\end{array}$ \\
\hline 8 & $\begin{array}{l}>10 \mathrm{~cm} \text { depth } \\
\text { with ice layers }\end{array}$ & 530 & 40 & ‘Skipped' - no shear. \\
\hline 9 & $\begin{array}{l}>10 \mathrm{~cm} \text { depth } \\
\text { with ice layers }\end{array}$ & 630 & 48.75 & $\begin{array}{l}95 \mathrm{~kg} \text { weight on top } \\
\text { of frame - frame rivet } \\
\text { sheared. }\end{array}$ \\
\hline 10 & $\begin{array}{l}>10 \mathrm{~cm} \text { depth } \\
\text { with ice layers }\end{array}$ & 630 & 52.5 & $\begin{array}{l}95 \mathrm{~kg} \text { weight on top of } \\
\text { frame. }\end{array}$ \\
\hline 11 & $\begin{array}{l}>10 \mathrm{~cm} \text { depth } \\
\text { with ice layers }\end{array}$ & 630 & 57.5 & $\begin{array}{l}\text { Possible pre-fracture - } \\
95 \mathrm{~kg} \text { weight on top of } \\
\text { frame. }\end{array}$ \\
\hline 12 & surface layer & 530 & 300 & $\begin{array}{l}95 \mathrm{~kg} \text { weight on top of } \\
\text { frame. }\end{array}$ \\
\hline 13 & $\begin{array}{l}>10 \mathrm{~cm} \text { depth } \\
\text { with ice layers }\end{array}$ & 530 & 80 & $\begin{array}{l}95 \mathrm{~kg} \text { weight on top of } \\
\text { frame - only } 2 \text { of } 3 \text { sec- } \\
\text { tions mobilised. }\end{array}$ \\
\hline
\end{tabular}

'skipping' etc.) an attempt will be made here to assess the validity of the measured data and verify agreement with published or expected values. This will allow comparison of these values with CPT data at a later stage.

Note that the shear strength discussed here is merely a measure of the cohesion of the snow, and does not measure any additional resistance due to compaction (as within a compressive test or CPT); no densification occurs during the test. This distinction is further clarified by Perla et al. (1982), and is consistent with Mellor's observations (Mellor, 1975).

Results presented in Table 6.2 reveal considerable scatter in data from a homogeneous layer, consistent with the problems noted in testing. The value obtained in the one 'good test' $(17.5 \mathrm{kPa})$ is of similar magnitude to the range of shear strengths noted within Mellor (1975) for snow of density $390 \mathrm{~kg} \mathrm{~m}^{-3}$ and may be representative of the actual shear strength.

If the results of Table 6.3 and Figure 6.8 (densities 465 to $630 \mathrm{~kg} \mathrm{~m}^{-3}$ ) are com- 


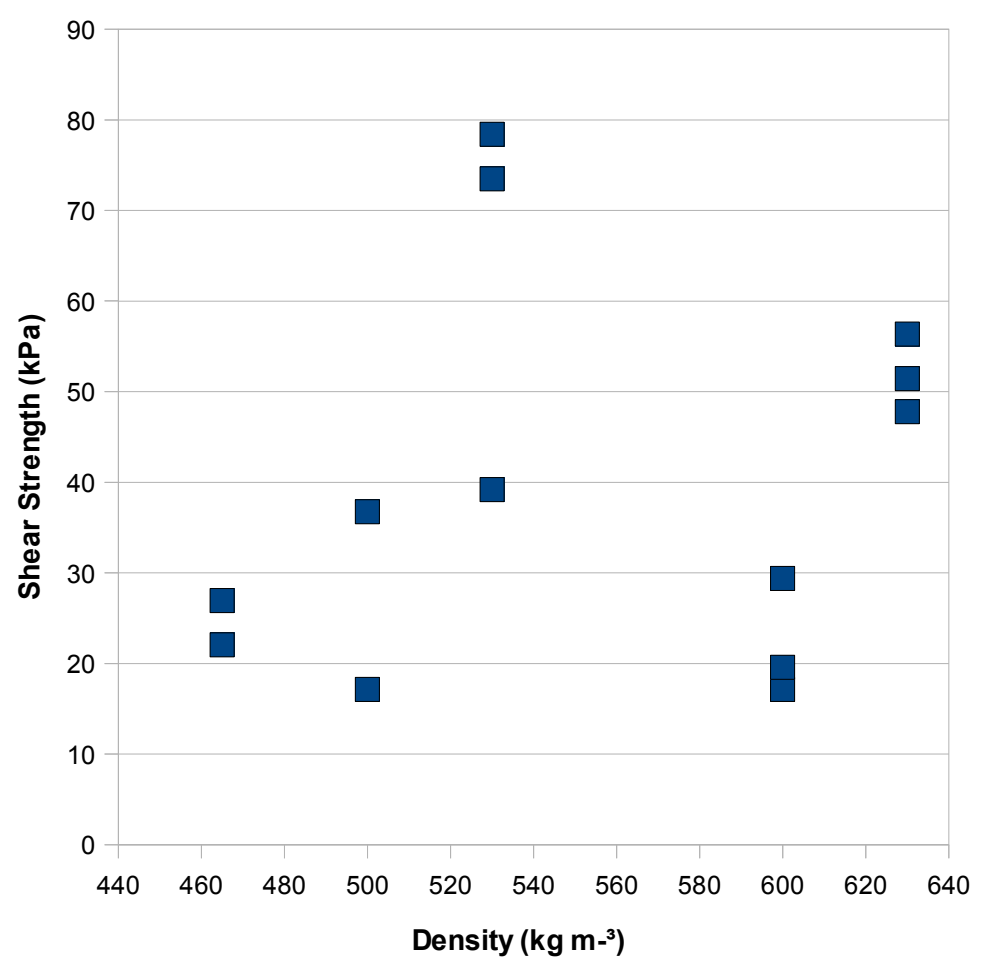

Figure 6.8: Shear strength versus density from testing conducted in the Halley V vehicle park. A large amount of scatter is evident.

pared to this same reference (Mellor, 1975) they fall short, by an order of magnitude. However, they are consistent with values suggested within Perla et al. (1982), which shows deviation from Mellor's collected results at a density of $\sim 350 \mathrm{~kg} \mathrm{~m}^{-3}$. Perla et al. (1982) note that the higher strength values presented by Mellor are largely obtained from laboratory testing of aged samples, hence they may not be representative of field specimens. The snow within the vehicle park although of high density had only recently been disaggregated and compacted (within the last week) hence limited bonding may have developed (snow of density $500 \mathrm{~kg} \mathrm{~m}^{-3}$ may take up to 50 days to develop maximum strength at a temperature of $-10^{\circ} \mathrm{C}$ (Abele, 1990)). Many of the layers tested exhibited ice layers within the sheared blocks, potentially providing a plane upon which shearing may preferably occur.

Table 6.3 shows that a number of these tests were conducted with a $95 \mathrm{~kg}$ mass on top of the shear frame in order to encourage shear behaviour rather than 'skipping' or partial fracture. Although this increased normal force would be expected to increase the frictional resistance of the snow to shear after fracture, it has limited impact on the cohesive strength of the snow hence this additional loading is ignored; instead, tests under such loading probably more closely reveal an accurate strength index.

The presented shear strength values although exhibiting much scatter, may provide representative shear strength values. These values are compared with CPT data in the next chapter. 


\subsection{Pressure Bulb Testing}

To assist in investigating the relationship between CPT data and surface bearing capacity, numerous attempts were made to determine the vertical extent of the pressure-bulb from a surface load. Data from this testing are presented here, and this relationship will be further discussed in Chapter 8. As previously described, this testing proved problematic, largely because of limitations of the equipment used and testing methods, hence limited data of value were obtained. The majority of tests were conducted using the thirteen tonne John Deere tracked-tractor, shown in Figure 6.9.

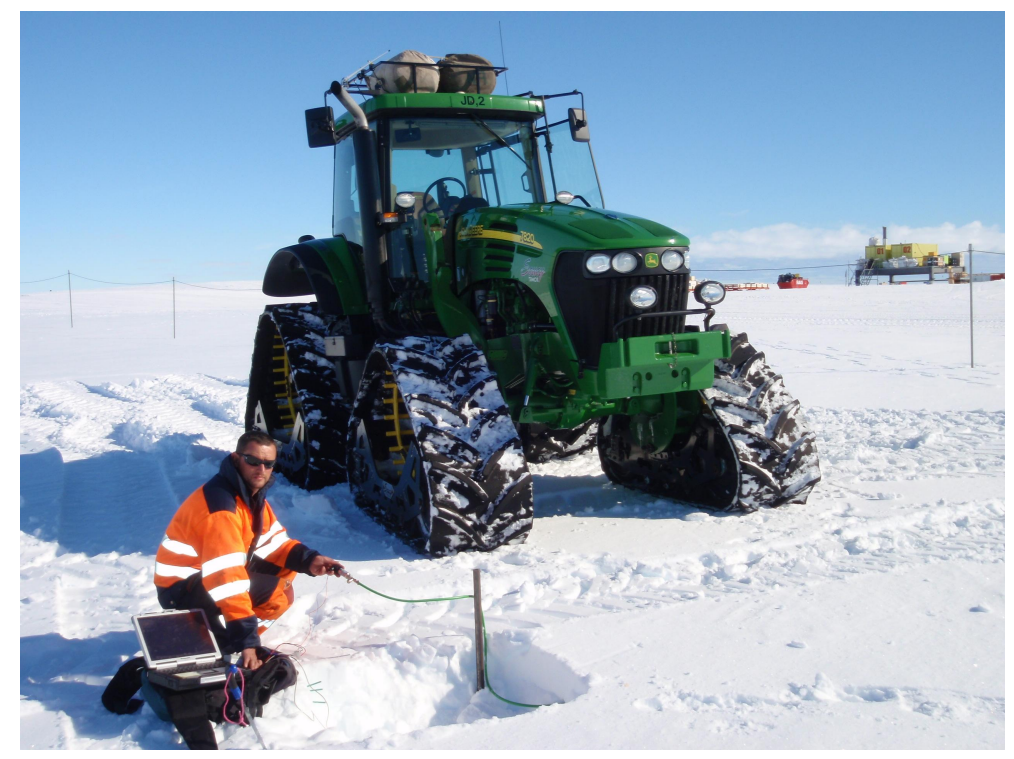

Figure 6.9: John Deere 7820 tracked-tractor used in pressure bulb tests.

Figure 6.10 presents pressure data obtained on 29 December 2009 in an area of virgin snow adjacent to the vehicle park. Voltage output from the pressure sensor is normalised with respect to the estimated bearing pressure under a single front track of the tractor of $27.4 \mathrm{kPa}$ and the curve is smoothed. This produces a curve which appears consistent with previous work conducted by Stehle (1970) on the vertical penetration of stress within snow, also shown in Figure 6.10.

Figure 6.11 presents the results from testing undertaken on 10 Feb 2010 in the snow-pack adjacent to snow pit \#1. All tests presented show the change in pressuremeter voltage (pressure) measured at various depths under the track or tracks of the John Deere tractor as it moved forward at $\sim 1 \mathrm{~m} \mathrm{~s}^{-1}$.

Because only one pressure measurement could be obtained (at a specific depth and location) during each tractor pass, the sensor was moved a short distance laterally along the pit wall or inserted to a greater distance in order to allow additional testing within uncompacted snow. Although Figure 6.10 presents useful data, additional testing as presented in Figure 6.11 suggests that limited value can be extracted from such data. Gathered data is briefly interpreted below.

Averaged data presented in Figure 6.10 are presented in tabular form in Table 6.4; 


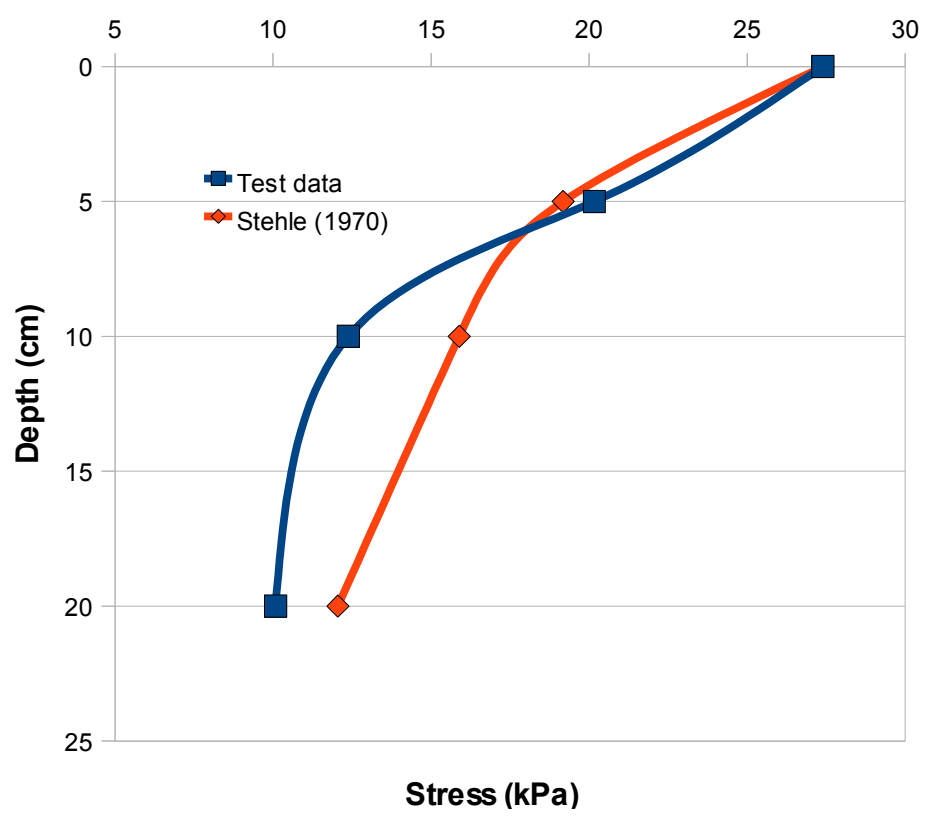

Figure 6.10: Pressure variation under John Deere tractor front track plotted against vertical stress distribution data after Stehle (1970).

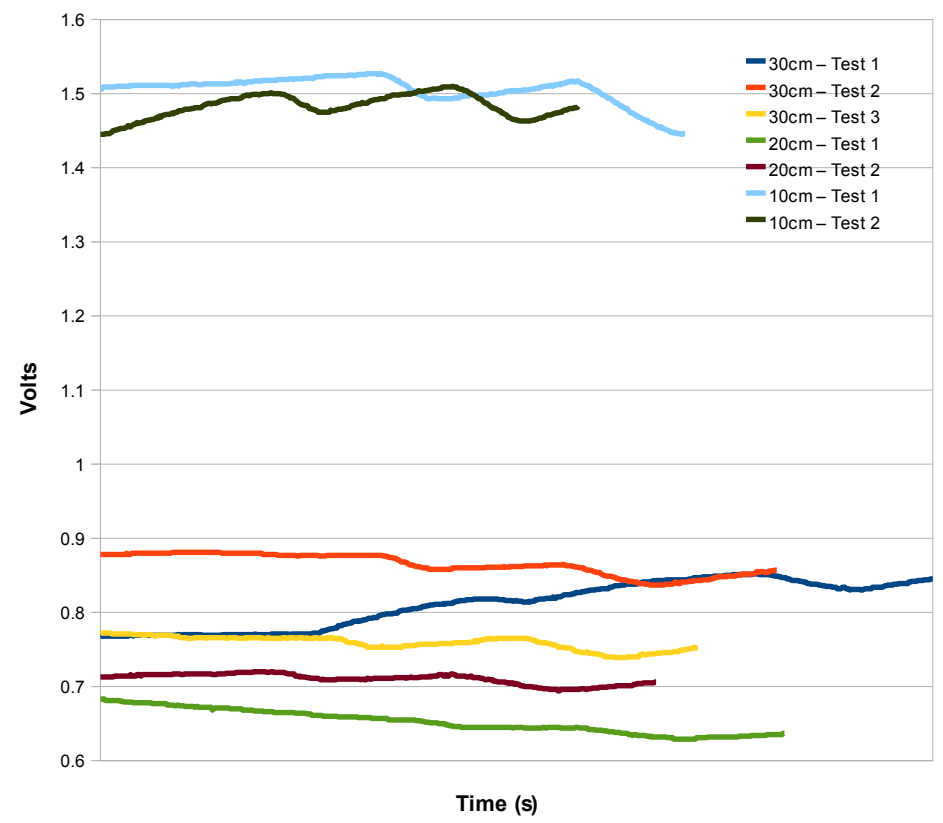

Figure 6.11: Pressure variations under John Deere Tractor, front and rear tracks. Testing conducted adjacent to snow pit \#1.

included is percentage pressure at depth, based on an assumed surface load of 27.4 $\mathrm{kPa}$.

Table 6.4: Pressure vs Depth.

$\begin{array}{ccc}\text { Depth (cm) } & \text { Pressure (kPa) } & \text { \% of surface load } \\ 5 & 20.16 & 73.6 \\ 10 & 12.39 & 45.2 \\ 20 & 10.08 & 36.8\end{array}$


When these percentages are plotted against data derived from Stehle (1970) and Kondratyeva (1945) (both in Abele (1990)), then comparison can be made (Figure $6.12)$.

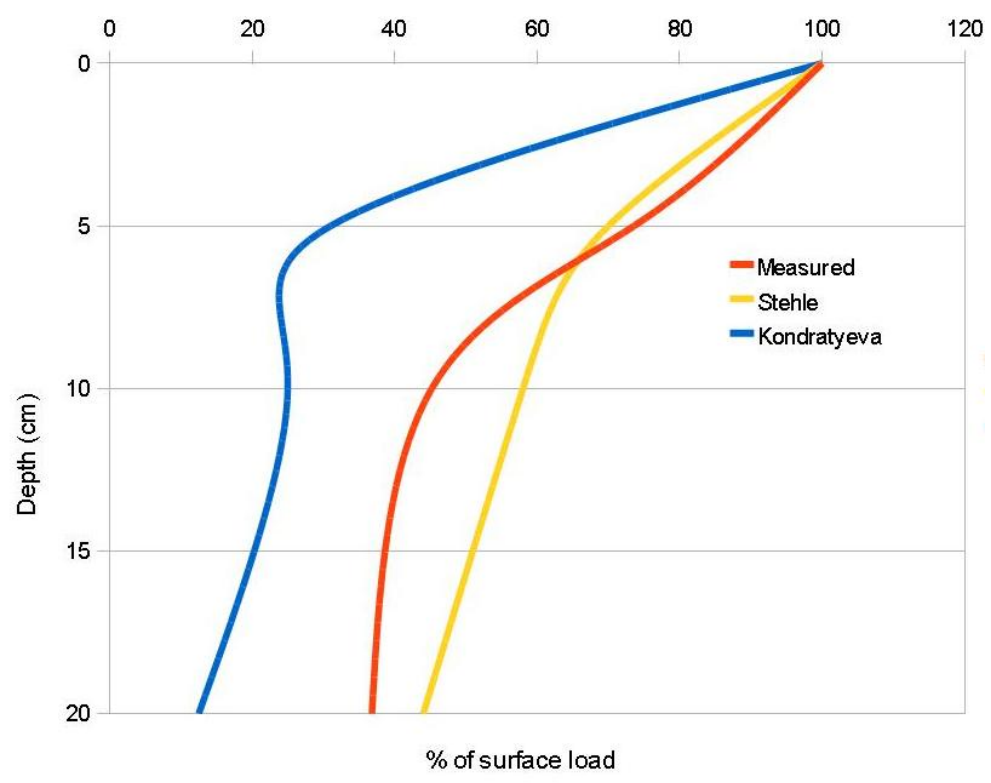

Figure 6.12: Halley pressure bulb data plotted against Stehle (1970) (measured) and Kondratyeva (1945) (modeled) data.

The curves presented in Figure 6.12 were obtained in snow of different densities and with different load parameters, hence variation in the shape, extent and magnitude of the resultant vertical stress distribution is expected. The percentage of surface load derived from Stehle decreases with depth in an almost linear manner whereas the graph constructed from Halley data shows percentage surface load decreasing in a power-law manner. However, this comparison suggests that the data acquired at Halley may provide representative vertical stress values.

The variation in magnitude of each test in Figure 6.11 is too limited to entail a thorough quantitative analysis, owing to either limited actual pressure transmission within the snow, or the limited ability of the pressure measuring equipment to accurately detect any variations. However, some comparison is made with recent results obtained from measuring vertical stress extent under tracked vehicles in snow (Pytka, 2009).

In a qualitative assessment of Figure 6.11, changes in pressure within the snow pack as the tractor's wheels pass over the sensor can be observed. As expected, the pressure value and measured variation is greater in the shallower tests. Inexplicably, the pressure measured in the two $20 \mathrm{~cm}$ tests is less than that measured within all tests at $30 \mathrm{~cm}$ depth, with pressure variation also appearing more limited.

Pressure data in Figure 6.11 are presented in raw voltage output because of the variations that were obtained whilst attempting to calibrate the pressure sensors. The most recent sensor calibration prior to this testing suggested that a variation in voltage 
of 0.01 volts is equivalent to a variation in vertical stress of approximately $20 \mathrm{kPa}$. This suggests that in the $10 \mathrm{~cm}$ tests pressure variations of approximately $60 \mathrm{kPa}$ were observed. This compares reasonably well with work by Pytka (2009) who at the same depth $(10 \mathrm{~cm})$ under a lighter $(\sim 8400 \mathrm{~kg})$ tracked grooming machine in snow of unknown density, recorded an average vertical stress variation of $\sim 25 \mathrm{kPa}$. The difference in vertical stress at $10 \mathrm{~cm}$ depth $(\sim 42 \%)$ compares reasonably well with the difference in the surface loading of the vehicles used $(\sim 29 \%)$.

The comparison above was between relative pressure increases within the snow; variation in voltage of $\sim 0.01$ volts was equivalent to a variation in stress of $\sim 20$ $\mathrm{kPa}$. However, absolute pressure calculations utilising the same calibration data appear problematic. A measured voltage of $\sim 1.5$ volts implies a weight on the sensor of $\sim 5 \mathrm{~kg}$ (assuming the extrapolated calibration curve remains linear). This equates to a surface weight of approximately $10 \mathrm{~kg}$ if the vertical pressure transmission is consistent with Figure 6.12. Based on an assumed sensor bearing area of $1 \mathrm{~cm}^{2}$ this surface weight of $10 \mathrm{~kg}$ equates to an estimated surface stress of $\sim 1000 \mathrm{kPa}$. This is far in excess of the estimated stress applied at the surface by the John Deere tractor, estimated to be only $\sim 14 \mathrm{kPa}$ (weight $\sim 13,000 \mathrm{~kg}, 40: 60$ load distribution front/rear and footprint of $\sim 1.86$ $\mathrm{m}^{2}$.) An absolute assessment of applied vertical stress using these methods appears unreliable, most probably because of the inaccuracy of relating surface calibration data to field measurements, and the inability to 'zero' the instrument once in place. Therefore, utilising the pressure sensor in an 'absolute' sense does not appear valid, however, 'relative' measurements appear consistent with expected variations.

It is not the purpose of this thesis to intimately examine the variation of stress within the snow pack beneath variable surface loading. However, collected data appear consistent with published data and serve to verify the expected extent of vertical stress distribution within the snow at Halley V. This may allow more accurate prediction of surface bearing capacity via CPT. The efficacy of this testing in assisting in the estimation of surface bearing capacity is further discussed in Chapter 8.

\subsection{Ground Penetrating Radar (GPR)}

Various GPR transects were performed in order to compare the radar signal with snow resistance measured via CPT, and to examine the suitability of GPR for spatially extrapolating stratigraphy and resistance data identified via CPT. GPR surveys were generally conducted just prior to or post CPT so that immediate comparison between the two methods could occur.

Figure 6.13 shows a short GPR trace over a distance of $\sim 2 \mathrm{~m}$ to a depth of 5.4 $\mathrm{m}$ obtained within the Halley $\mathrm{V}$ vehicle park. It is in line-scan format with modified colour table and display gain, and with returns from the antennae when stationary removed. The GPR was in time-scan mode, and depth in metres on the vertical axis is 
generated from nanosecond signal data using an average dielectric constant of 1.9. Distinctive horizons are evident and spatially consistent and allow ready comparison with collocated CPT data (Tests 1 \& 5), superimposed. Initial examination of this figure suggests limited agreement between peaks in cone tip resistance and layers detected via GPR.

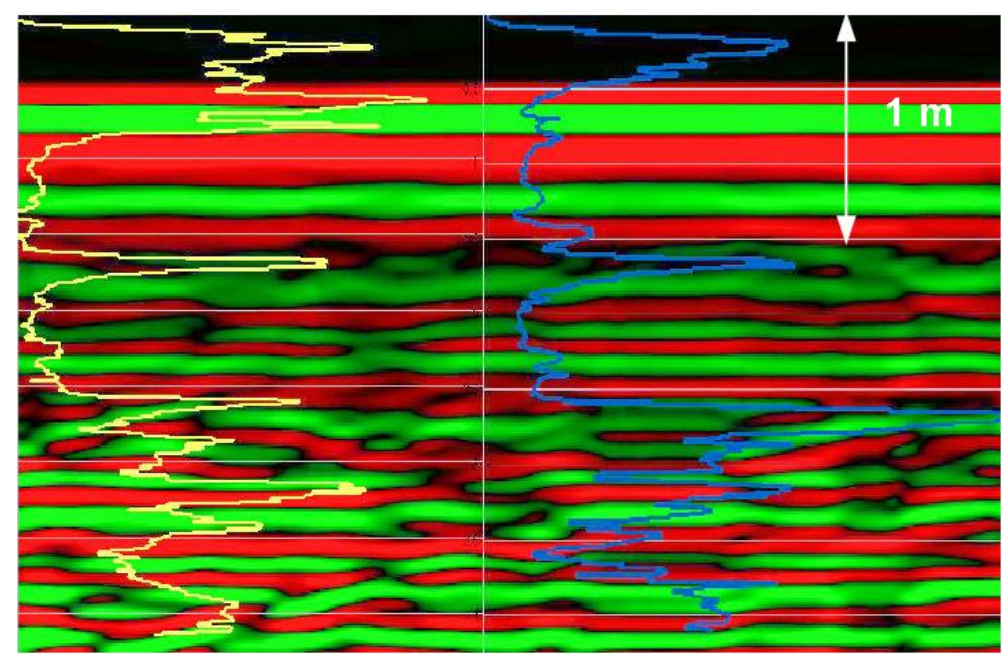

Figure 6.13: Cone tip resistance for Tests 1 (yellow) \& 5 (blue) to a depth of $\sim 3 \mathrm{~m}$ superimposed over collocated GPR linescan.

In analysing a GPR trace, the brightness of the return is proportionate to the difference in dielectric constant between layers; returns suggest interfaces between layers, not the layers themselves. Dark bands (green in Figure 6.13) are generally areas of lower reflection or limited variation in dielectric contrast whereas lighter areas (red in Figure 6.13) are typically areas of higher reflection where the difference in dielectric constant is greatest. Hence in Figure 6.13 it is expected that peaks in the CPT trace (suggesting harder material) may occur below the red bands which indicate the commencement of a denser layer with a higher dielectric constant. Further comparison of a static GPR trace in the vicinity of snow pit \#2 with CPT \# 62, 63 \& 64 is made in Figure 6.14.

Clearer comparison can here be made between the two measures; tip resistance typically peaks within the transition from green to red, or upon commencement of a red 'layer'. It should be reiterated that depth data derived from the GPR time data is dependant upon the selected dielectric constant (in this case 1.9, based on the average snow density of pit \# 2) and modification of this constant during post-processing to better represent actual density variations could provide more accurate comparison between GPR and CPT data. Further qualitative comparison can be made in Figure 6.15 where manually extracted amplitude data from a GPR 'wiggle' trace is directly compared with flat-plate resistance data from Test 6 over a limited depth.

The depth of layers can be estimated from the GPR trace by considering the depth mid-way between amplitude extremes, and it can be seen that this supposed change 


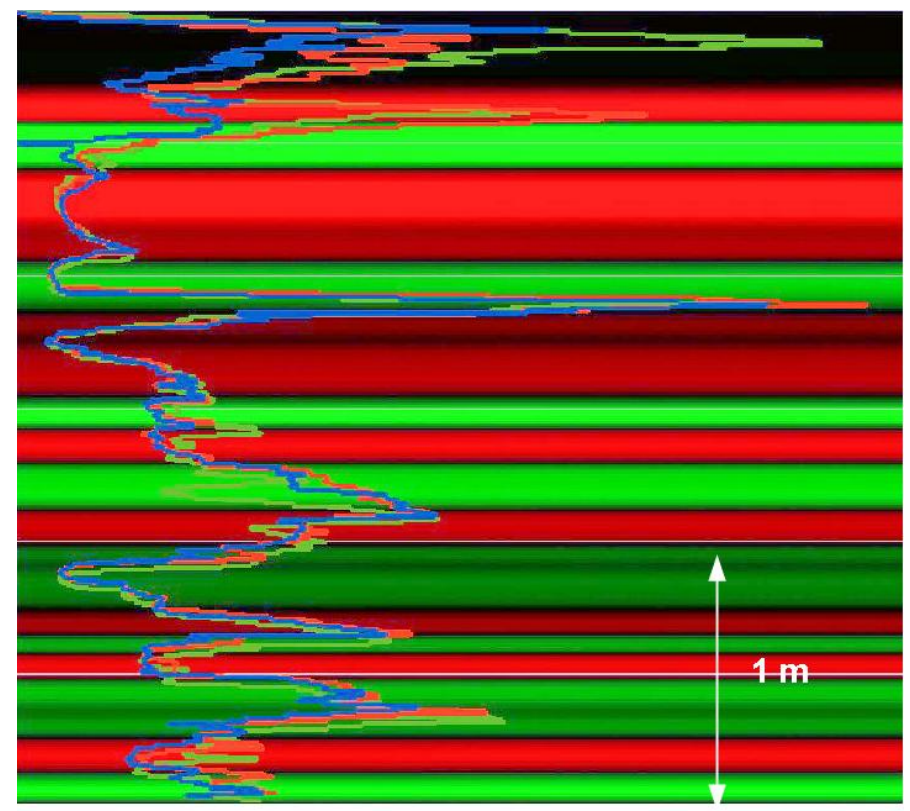

Figure 6.14: Cone tip resistance for Tests 62 (red), 63 (blue) \& 64 (green) to a depth of $\sim 3 \mathrm{~m}$ superimposed over collocated GPR linescan.

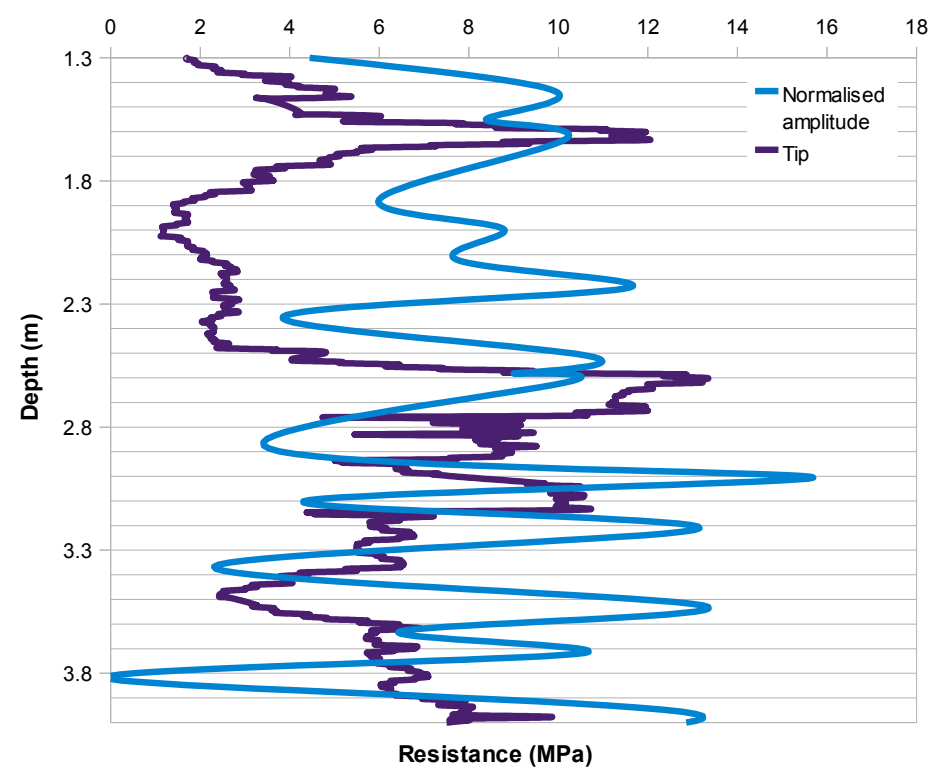

Figure 6.15: Cone tip resistance for Test 6 compared with manually extracted GPR amplitudes. GPR amplitude gradient and tip resistance are positively correlated.

of layer often coincides with the commencement of an increase or decrease in CPT resistance. This is as expected. Comparison can clearly be made between the two methods and a relationship can be observed. A statistically significant (at the $98 \%$ level, $\alpha>0.02)$ positive correlation $(\sim 0.505)$ was found between GPR amplitude gradient and tip resistance; this also showed tip resistance lagging behind the layer transition which is also expected owing to the penetration distance required for the cone tip to realise actual layer resistance. These results suggest a quantitative relationship between these two parameters. 
Obtaining a strong correlation between these two parameters is complicated because of:

1. difficulty in ascertaining exactly when the cone enters a layer,

2. inability of the cone to attain steady-state resistance within thin layers,

3. the nature of the GPR signal, in that amplitude is not indicative of layer composition but of the difference between layers,

4. the nature of the GPR signal, in that not all strata are resolvable via the 400 $\mathrm{MHz}$ antennae used,

5. the range of frequencies within the GPR data (centred about $400 \mathrm{MHz}$ ), and

6. the contradiction (when slicing radar amplitude with depth) between slicing thickly to obtain the complete radar waveform in order to more accurately identify interface depth versus the need to slice thinly in an attempt to identify thin strata (personal communication L. Conyers, 29 November, 2010).

Ideally, quantitative analysis of the radar amplitude to depth would combine a range of slicing and averaging methods in order to extract the most complete data from the GPR signal. Marshall et al. (2007) in comparing a $818 \mathrm{GHz}$ FMCW radar and the SMPT, both higher resolution instruments, stated that "major stratigraphic horizons could be followed along radar profiles and identified in SMPT measurements" and that "a very thin hard crust $(0.2-0.4 \mathrm{~mm})$ that was continuous caused strong signals that were identifiable in both the SMPT and the radar measurements at five different sites along a $10 \mathrm{~m}$ traverse." Although Marshall et al. (2007) were able to confirm the qualitative relationship between penetrometer and radar data, even with the use of higher resolution equipment, quantitative comparison proved problematic. The GPR appears an effective tool for spatially extrapolating snow mechanical data as obtained by CPT. However, although briefly explored above, further work is necessary to enable reliable quantitative comparison of these methods.

\subsection{Summary}

This concludes discussion of the supplementary subsurface testing that was conducted in support of my CPT investigations. The next chapter draws upon results from this chapter to investigate and verify methods of determining both snow stratigraphy and strength from CPT data in polar snow. 


\section{Chapter 7}

\section{Stratigraphy \& strength from CPT}

Lunne et al. (1997) say that in the absence of groundwater, the two main objectives of any subsurface exploration program is to firstly: determine the "nature and sequence of the subsurface strata" and secondly: determine the "physical and mechanical properties of the subsurface strata." This chapter aims to address these objectives.

Most of the discussion thus far has been empirical in nature but this chapter explores the physical mechanisms at play during CPT and examines how they may be modelled to assist in our interpretation of the processes at work.

\subsection{CPT and stratigraphy}

In the previous chapter results were presented that empirically verified a link between observed snow stratigraphy and cone tip resistance and sleeve friction measurements. Previous discussion suggests that sensing ahead of the cone in dry polar snow appears negligible and that no compacted zone occurs ahead of the $60^{\circ}$ cone. Thus, when interpreting snow stratigraphy from CPT data, no allowance needs to be made for deviation of the tip resistance trace prior to entering a layer, as may be necessary in many soils. However, once the cone has entered a layer, in order for actual layer resistance to be determined, the cone must penetrate into the layer by an amount described as the "critical depth" (De Beer, 1963). This topic is covered at length with respect to calcareous soils by Evans (1987) in his $\mathrm{PhD}$ thesis. A quantitative outcome of this is that although the CPT may start to register an increase in resistance upon penetration of harder layers, the resistance of such layers could be underestimated (Lunne et al., 1997) with the converse occurring in softer layers.

In the CPT at Halley this critical depth (within a layer) was rarely reached so that the observed resistance trace rarely exhibits a steady-state value consistent with the true resistance value of a snow layer, but instead constantly rises and falls as new harder or softer layers are encountered.

Figure 7.1 (showing selected data from Test 9) depicts this problem, with overlaid red bars depicting possible boundary locations (stratigraphy) where the gradient of tip 
resistance with depth equals zero $\left(d q_{c} / d z=0\right)$.

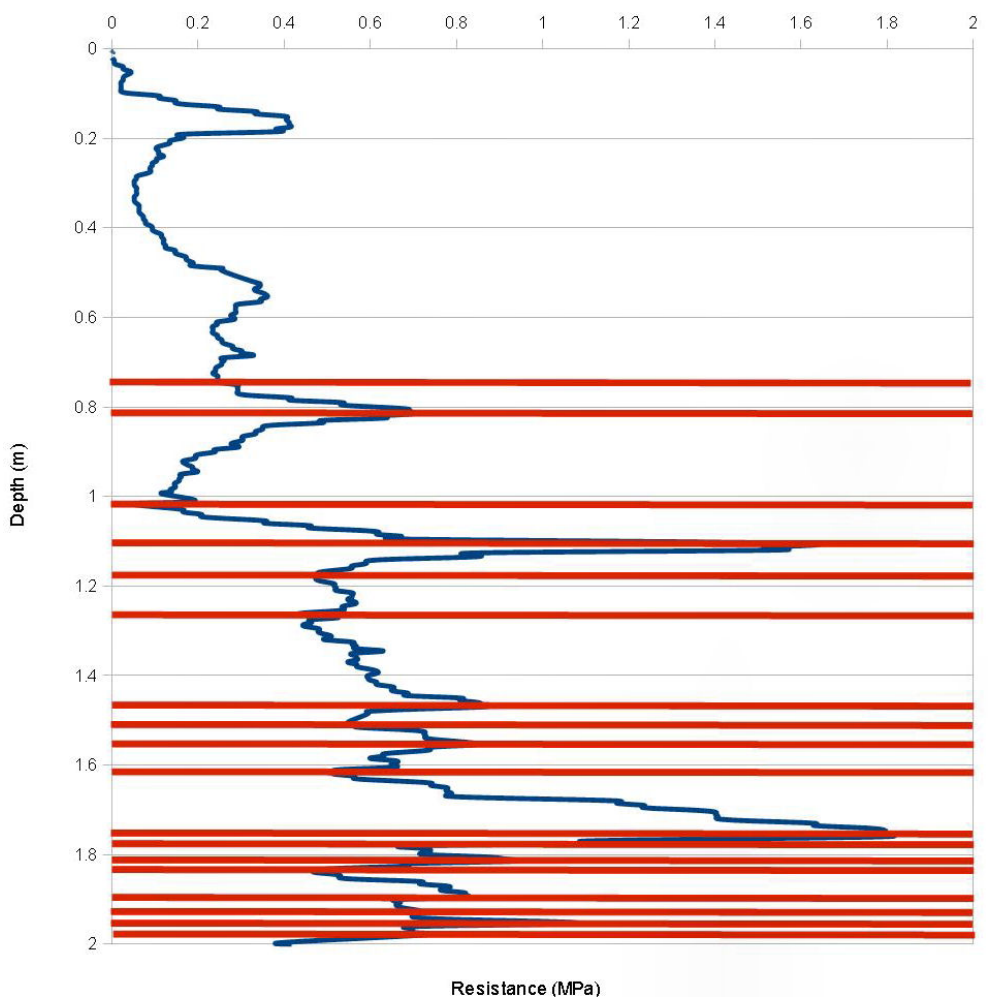

Figure 7.1: Tip resistance trace is constantly varying because layer thicknesses are typically less than the 'critical depth' required for the cone to return a steady-state resistance. Where is the layer boundary?

Controlled lab experiment tests similar to Evans (1987) are likely to be necessary to effectively establish the critical depth. It is unlikely that the field data presented herein will enable precise calculation of such a parameter. However, some investigation is warranted. It is useful to try and estimate some measure of what this "critical depth" of insertion within a layer might be before attempting to estimate strata from CPT data.

De Beer (1963) showed for sand that the critical depth for a two-dimensional wedge (rather than a three-dimensional cone) of diameter $\sim 40 \mathrm{~mm}$ was approximately $90 \mathrm{~cm}$. However, this theory was based on Meyerhof's general shear failure theory (Meyerhof, 1951) and is not relevant to the punching shear failure anticipated in snow. Evans (1987) in his tests on model piles in calcareous soils found that a penetration of four pile diameters needed to occur before a steady state resistance was obtained, but his tests were with a blunt-ended pile that generated a significant compacted zone ahead of it. Perhaps somewhat relevant to the penetration of the flat plate in snow, but not the cone.

Johnson (2003) in his work on penetration within bonded granular materials states that the depth $\left(\mathrm{Z}_{\max }\right)$ at which the cone is fully engaged with the granular material is given by: 


$$
Z_{\text {max }}=\frac{r_{b \max }}{\sin \theta \cos \theta}
$$

where $r_{\text {bmax }}$ is the base radius for a cone penetrometer and $\theta$ is cone penetrometer half angle. For the $36.7 \mathrm{~mm}$ cone used at Halley this results in an engagement distance of $42.38 \mathrm{~mm}$, or $\sim 1.15$ times cone diameter; a "critical depth" of the order of the cone diameter appears physically appropriate.

Pielmeier and Schneebeli (2003) discussing the measurement of stratigraphy using the $5 \mathrm{~mm}$ diameter snow micro penetrometer state that the vertical resolution of the cone is $1 \mathrm{~mm}$ "because it is the upper millimeter of the measuring tip that contains about $75 \%$ of the cone area on which most of the penetration resistance acts." Lutz et al. (2007) in their assessment of stratigraphy using the SMPT and statistical methods, suggested that transition zones of dimension approximately equal to the length of the cone head (equivalent to $\sim 85 \%$ of cone diameter in a $60^{\circ}$ cone) exist where the cone head is entering or exiting a weak (or hard) layer. Bellaire et al. (2009) elaborate on this and suggest that "the layer resolution of the SMPT corresponds to the height of a truncated cone with a lateral area that is two-thirds of the lateral surface area of the whole cone." These observations suggests that for a standard CPT cone of $36.7 \mathrm{~mm}$, rather than a "critical depth" existing that is some dimension greater than the cone diameter (such as in soils), perhaps the actual resolution of the cone is some percentage reduction of the cone diameter, say $\sim 25 \mathrm{~mm}$. This would mean that the $36.7 \mathrm{~mm}$ cone, an oft' considered 'unwieldy' instrument may actually be able to provide valuable resolution in hard polar snow packs. An approximate value of this critical depth (circa $25 \mathrm{~mm}$ ) can now be used in an attempt to establish strata from a CPT tip resistance trace.

Figure 7.2 shows an excerpt from Test 9 where tip resistance has been averaged over this $25 \mathrm{~mm}$ distance. Possible stratigraphic features determined using this critical depth are also shown (terminology consistent with that used by Lutz et al. (2007)).

The five points nominated in this figure are:

1. Weak layer (defined first; middle defined by the minimum value in the resistance profile),

2. Superstratum (defined 2nd; adjacent maximum, where the superstratum resistance begins to subside into the weak layer),

3. Substratum (defined 2nd; adjacent maximum, where the substratum resistance begins to subside into the next weak layer),

4. Upper transition (defined last; position between the apparent upper boundary (2) and the weak layer center (1)), and

5. Lower transition (defined last; position between the weak layer center (1) and the apparent lower boundary (3)). 


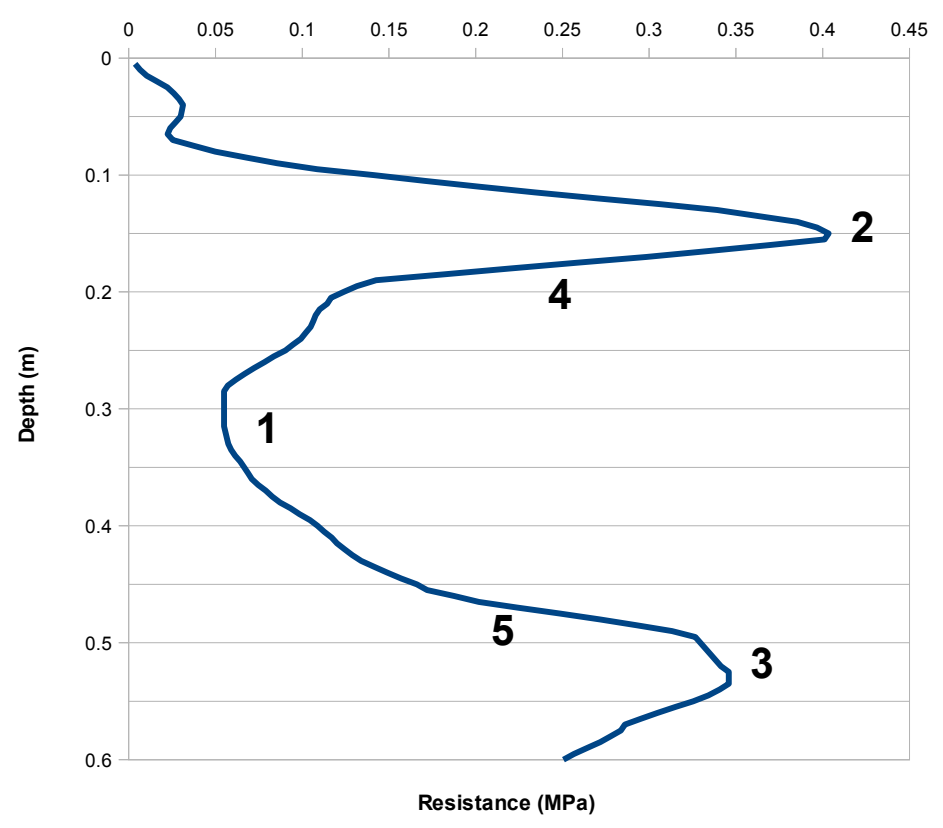

Figure 7.2: $25 \mathrm{~mm}$ running average of tip resistance versus depth and probable location of layer boundaries via utilisation of critical depth.

Knowing the supposed critical depth, this process can theoretically be applied to any tip resistance trace to infer probable layer boundaries; automation of this technique via statistical interpretative means may be preferable. Figure 7.3 shows the application of this technique to the complete tip resistance data from Test 9 so that a comparison of observed and generated strata can be made.

This method can be tuned by altering the distance over which the gradient is calculated, or by neglecting any discontinuous gradients. Figure 7.3 shows that some layers are picked up very well by this method. However, at other depths, observed layers are missed, or layers are suggested at different depths. This method appears promising but further work is required before stratigraphy can accurately and consistently be resolved solely from CPT tip resistance data. A further tool that may assist in stratigraphy resolution is the unique incorporation of the friction sleeve data.

\subsubsection{Incorporation of friction sleeve to resolve stratigraphy}

The novel utilisation of a friction sleeve within a penetrating cone in snow may provide valuable insight into snow strata, amongst other things. Figure 7.4 shows average tip resistance and normalised average sleeve friction versus depth for Test 9. Both values have been vertically averaged (over the cone length and friction sleeve length respectively) and adjusted for lag (by cross-correlating the two parameters).

In this figure, very good agreement can be seen between the two traces. However, better comparison can be made by superimposing these values over stratigraphy derived from Snow Pit \#1; stratigraphy represented by arbitrarily normalised density values 


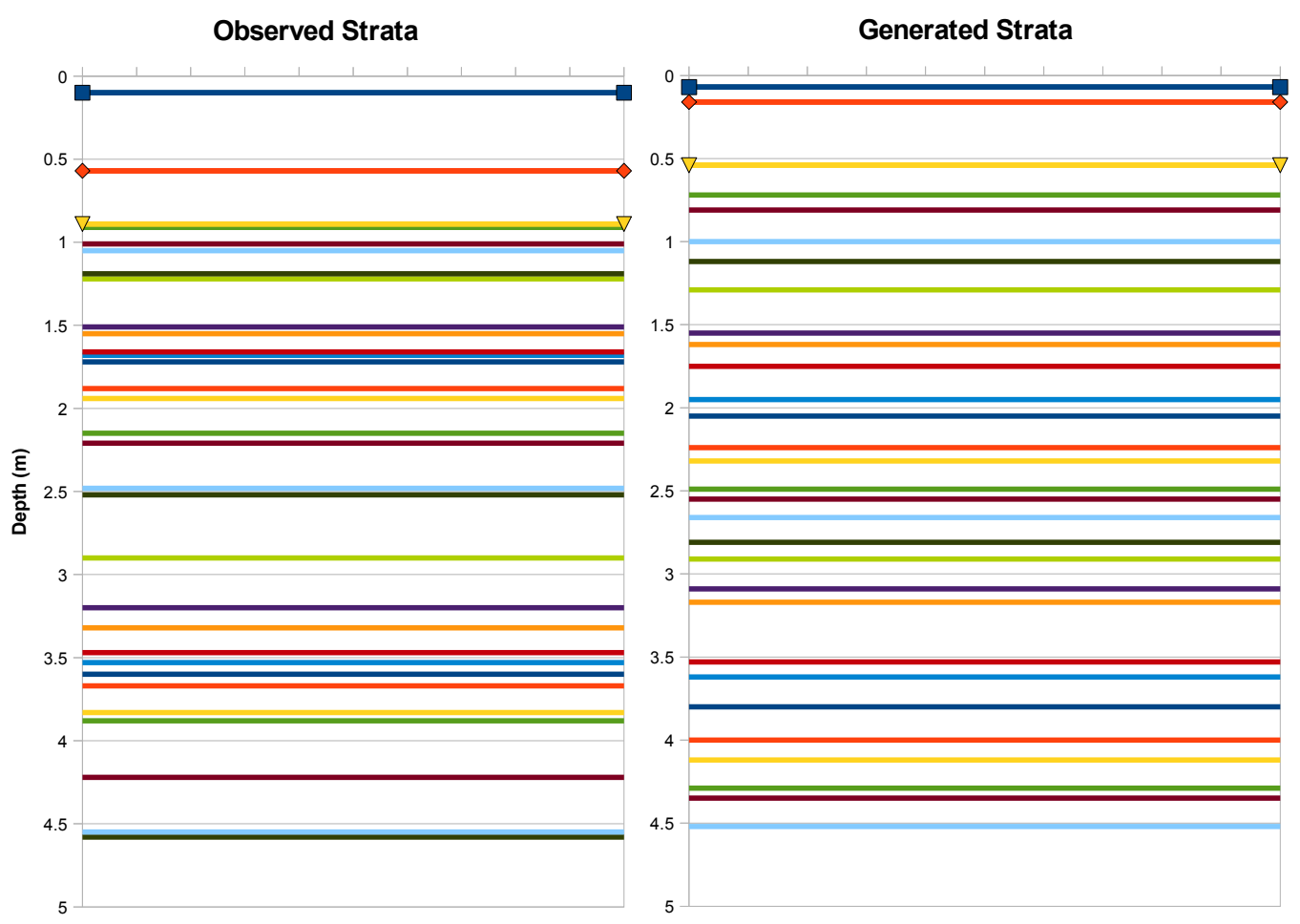

Figure 7.3: Comparison of observed stratigraphy from Snow Pit \# 1 with stratigraphy generated from the CPT tip resistance data after considering gradient change of tip resistance averaged over the calculated critical depth. Note: colours alternate sequentially and do not indicate identical layers.

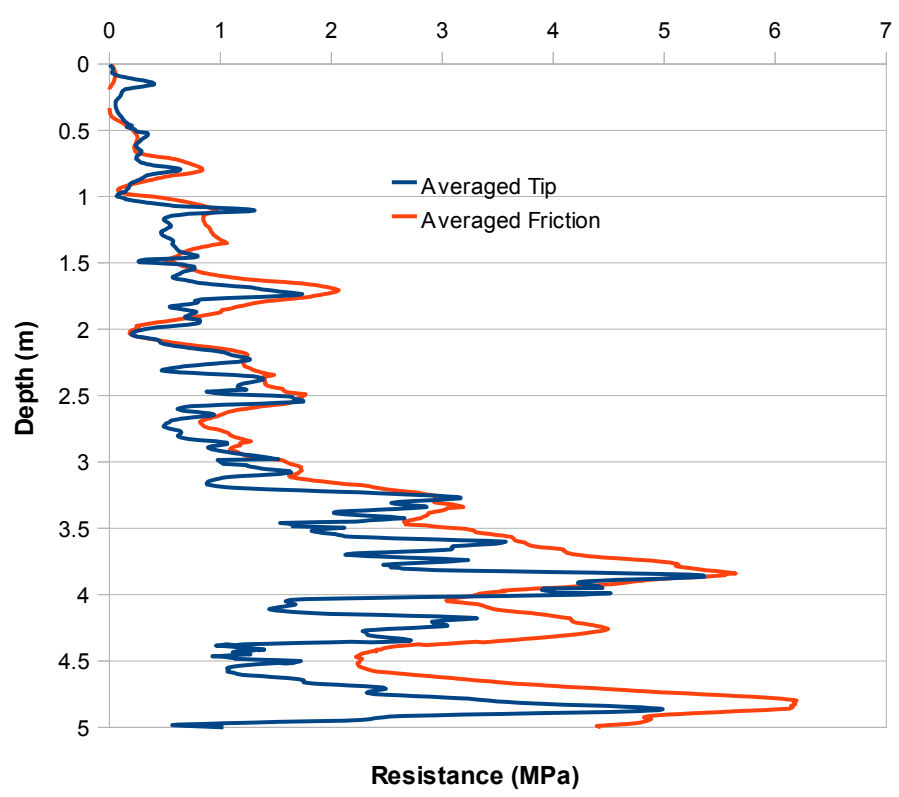

Figure 7.4: Average tip resistance and normalised, averaged and lag-adjusted sleeve friction versus depth (Test 9).

(Figure 7.5).

Now, ready comparison between CPT data and snow stratigraphy can be more easily made. Qualitatively it is seen that both tip resistance and friction vary with 


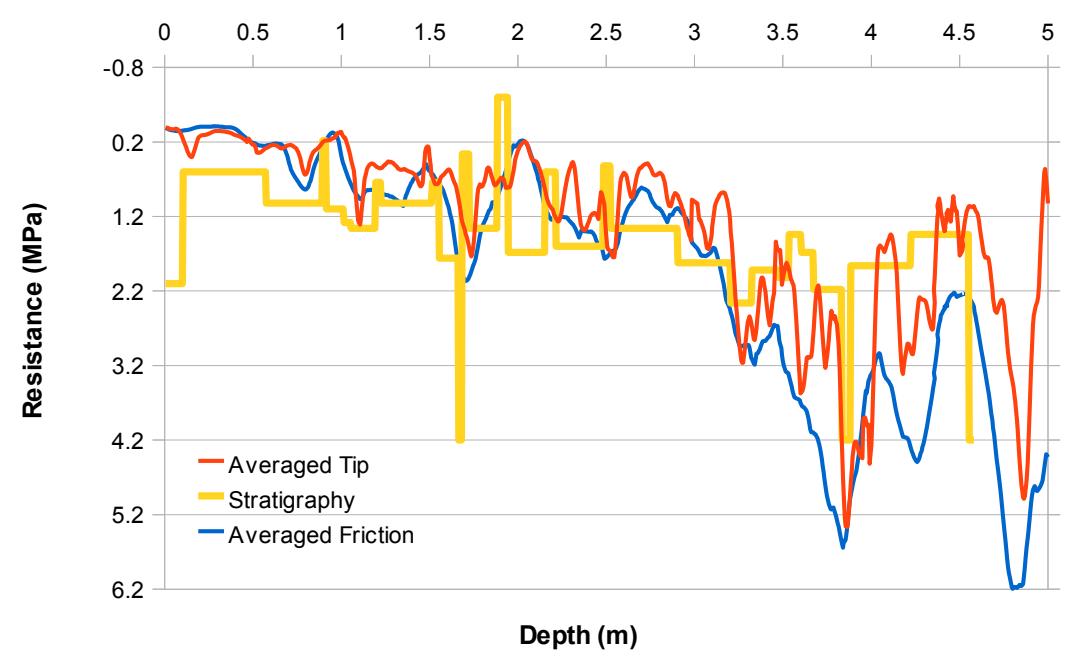

Figure 7.5: Average tip resistance (Test 9) versus normalised, averaged and lagadjusted sleeve friction (Test 9) superimposed over stratigraphy, defined by density from snow pit \#1. Both tip resistance and friction vary with stratigraphy.

stratigraphy, although tip resistance alone reproduces many of the peaks, expected because of the large friction sleeve; some misalignment is apparent (notably between 2.0 and $2.5 \mathrm{~m}$ ) perhaps owing to measurement error during snow pit analysis. However, generally tip resistance is seen to depict stratigraphy well. This qualitative assessment suggests the following:

1. tip resistance starts increasing before a hard layer, at a distance similar to the estimated critical depth of $\sim 25 \mathrm{~mm}$,

2. tip resistance starts decreasing before a soft layer, at a distance similar to the estimated critical depth of $\sim 25 \mathrm{~mm}$, and

3. steady-state resistance is approached in thicker snow layers.

The addition of the friction sleeve information per se does not appear to greatly assist in stratigraphy resolution, other than to provide averaged verification of the tip resistance trace. It is valuable to compare stratigraphy derived from the CPT data with directly observed stratigraphy from a snow pit for validation purposes, but it appears unlikely that the CPT will provide more detailed profiling than that obtained via a slow and methodical manual approach. However, use of the CPT can bring great efficiency to such a task and may prove particularly useful in the rapid estimation of stratigraphy, especially in snow where layer thickness exceeds the critical depth $(\sim$ $25 \mathrm{~mm}$ ). Further application of statistical interpretative methods may increase the resolution of CPT-based methods but is not expanded upon here. 


\subsubsection{Stratigraphy resolution using a flat plate}

Comparison was also made between observed stratigraphy and tip resistance and friction sleeve measurements obtained using a flat plate. The following points of difference or relevance are noted:

1. the critical depth of insertion for the flat plate is expected to be less than that for the cone (likely $\sim 8-9 \mathrm{~mm}$ ), on the assumption that the flat plate will attract a compacted conical mass on its face (perhaps $\sim 10 \mathrm{~mm}$ in height) as penetration continues, and

2. this decreased critical depth is consistent with gradient analysis of flat plate versus cone traces which shows that upon entering a new layer the resistance trace for a flat plate increases at a 35 - $50 \%$ greater rate than the cone.

Because the critical depth is expected to be less when using a flat plate, snow stratigraphy may more easily be discerned. However, CPT with a flat plate will produce substantially different qualitative and quantitative results from those obtained with a cone, hence additional analysis and interpretation may be required before seeking comparison with conical CPT or strength proxy data.

\subsection{Variation with density}

The discussion above attempted to compare strata observed via manual analysis of a snow pit with the CPT trace. Such strata are typically defined by density and possibly hardness measurements and whilst density alone may not be an adequate sole descriptor for the mechanical behaviour or physical characteristics of a snow layer, it is readily understood and has been used historically. Variation of CPT data with density is therefore of interest.

Resistance may vary within snow of the same density because the microstructure of the snow is different, but microstructural changes typically accompany changes in density, hence variation of both tip resistance and sleeve friction with density is expected.

Figure 7.6 shows tip-resistance for Test 10 (flat plate) and snow density derived from snow pit \#1 plotted against depth, and Figure 7.7 shows sleeve friction (averaged over sleeve length and normalised to allow comparison) and density for Test 9 (cone) plotted against depth.

In Figure 7.6, three thin icy layers of density greater than $\sim 450 \mathrm{~kg} \mathrm{~m}^{-3}$ can be identified. Variation of both tip resistance and sleeve friction with density can also be observed in Figure 7.5 where tip resistance and sleeve friction are plotted against normalised density. Brief consideration of each of these with density is considered below. 


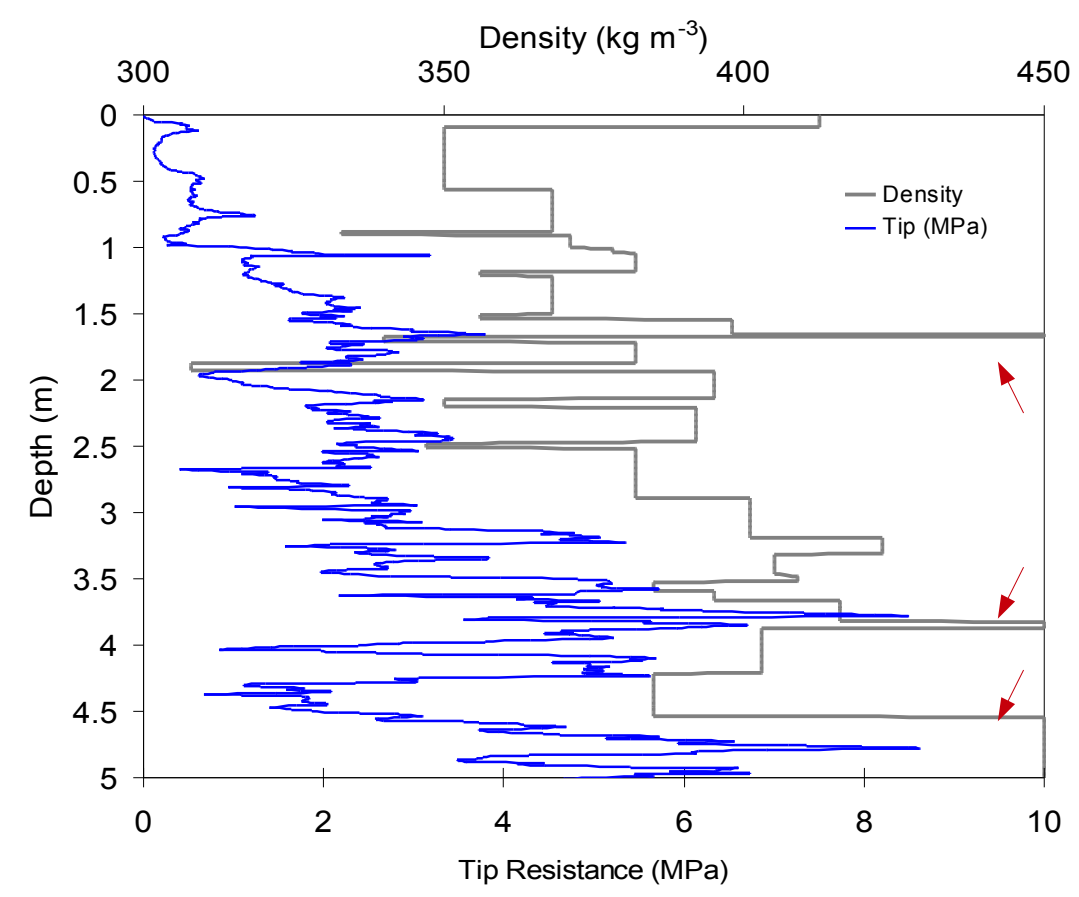

Figure 7.6: Variation of tip resistance and density with depth. Red arrows identify layers of ice discussed in text.

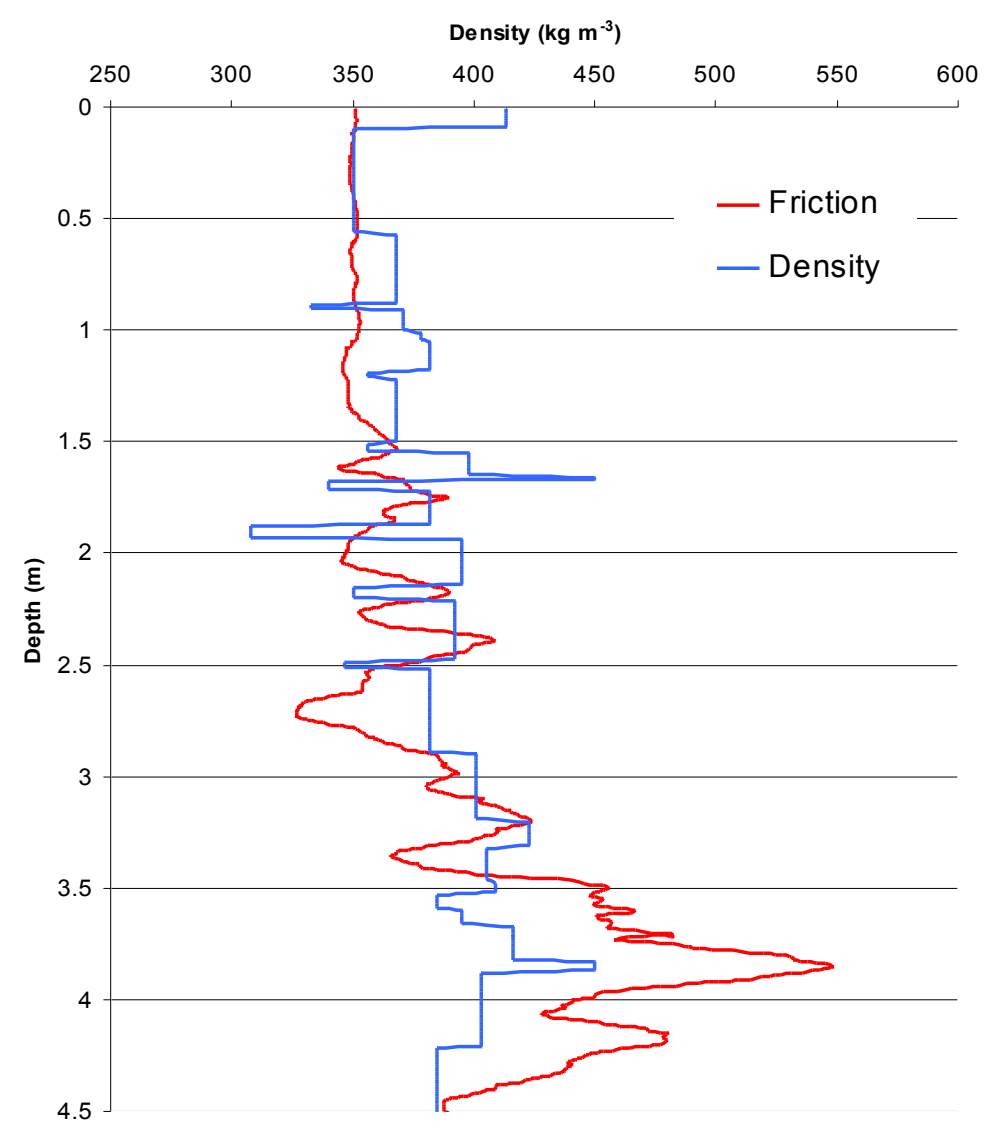

Figure 7.7: Normalised sleeve friction averaged over sleeve length (Test 9) and density versus depth. Sleeve friction appears to trend with density.

\subsubsection{Tip}

Figure 7.6 shows good qualitative correlation between tip resistance and density. Although the resistance trace varies within each layer (owing to the distance required 
to realise steady-state resistance because of the critical depth) variations in resistance agree well with density variations. Particularly hard and soft layers are evident, coinciding well with very dense and less dense layers, and both resistance and density are observed to generally increase with depth.

The increase of tip resistance with density is consistent with the positive correlation between ram hardness (obtained via the rammsonde, see Chapter 2) and density (Gubler, 1975) and is expected. Some resistance variation in snow of the same density is expected because density is not necessarily correlated with the level of bonding within the snow whereas measurements made by CPT (or other hardness measurements) will vary with such changes. Although a positive correlation between tip resistance and density is expected, quantifying this relationship from data such as that presented in Figure 7.6 is difficult; resistance data is recorded almost continuously whereas density is defined in thicker discrete layers because of sampling technique. Snow microstructural changes and cone size/shape affect the resistance data but cross-correlation between density and layer-averaged resistance reveals a significant positive correlation of $>0.6$. If the gradients of the density and resistance increase with depth are compared $(0.0182$ vs 0.1485 ), resistance is seen to vary by almost an order of magnitude more than density. This is consistent with the understanding that snow not only increases in density with depth, but that increased sintering and strengthening of bonds within the snow also occurs.

\subsubsection{Sleeve friction}

The relationship between friction sleeve data and density is perhaps more complex. Discussion in Chapter 5 suggested that sleeve friction may be positively correlated with density and with tip resistance. Tip resistance was shown to be positively correlated with density, thus a positive correlation between sleeve friction and density is probable.

Numerous authors (Casassa et al. (1991), Colbeck (1994)) have noted the decrease in kinetic friction with increasing density, and Mellor (1964) and Ericksson (1955) both note a decrease in friction with grain size. Grain size generally increases with depth during densification (although the increase to a depth of $5 \mathrm{~m}$ may only be of the order $\sim 0.1 \mathrm{~mm}$ (Rick and Albert, 2004)), hence some decrease in friction with increased density is implied. This is contrary to observations made via CPT.

Figure 7.7 shows that sleeve friction appears to trend with density. Lag in the friction trace is evident especially in the upper $2.5 \mathrm{~m}$, but this lag is expected and between $2.5 \mathrm{~m}$ and $4.5 \mathrm{~m}$ sleeve friction appears to vary with density. Both friction and density increase with depth albeit friction (similar to tip resistance) increases at a greater rate. Lag of $\sim 100 \mathrm{~mm}$ is evident, consistent with the physical dimensions of the friction sleeve, and the cross correlation between density and sleeve friction is $\sim$ 0.6 (Pearson correlation significant at the 0.01 level (2 - tailed)).

This observed increase in sleeve friction with density is assumed to be due to an 
increased normal force acting upon the friction sleeve caused by the greater difficulty in packing fractured particles at the cone shoulder as density of the snow undergoing penetration increases. Such a process would cause greater sleeve friction to be measured in higher density snow. A stylised representation of this process is shown in Figure 7.8.

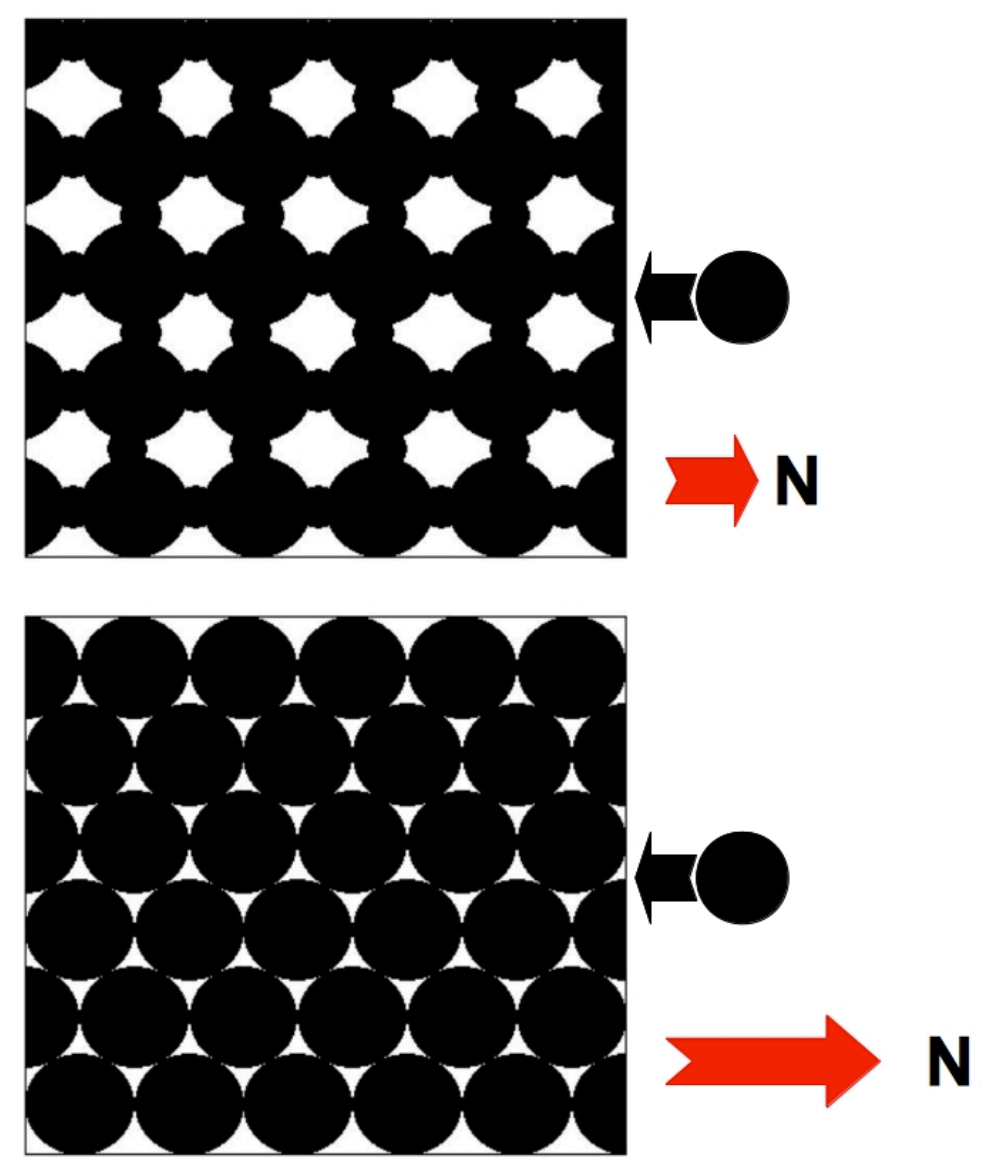

Figure 7.8: The normal force acting upon the cone is presumed greater in higher density snow where greater resistance opposes fractured particles displaced laterally by the cone; therefore, the measured sleeve friction is higher.

This supposition is supported by data presented earlier in Chapter 5 (Section 5.13, Figure 5.25) which showed that friction values in pre-drilled holes (where less mass will be packed) are substantially lower than standard friction values, suggesting the influence that normal force may have on sleeve friction values. Examination of flat-plate data presented by Abele (1970) also shows that as density increases, penetration resistance also increases and penetration distance decreases. These observations support the supposition that an increase in density will result in increased friction, not because of grain size variations but because of the increase in normal force acting on the sleeve.

Comparison of CPT resistance data (which are responsive to the effects of snow microstructure) with another snow strength proxy which is also responsive to microstructure (hardness) occurs next. 


\subsection{Comparison of tip resistance with hardness}

Hardness was briefly defined and considered with respect to other snow strength indices in Chapter 2. The CPT measures hardness directly hence comparison with other snow hardness data is of interest.

Figure 7.9 compares averaged cone tip resistance from Test 9 with the range of hand hardness obtained from snow pit \# 1, quantified according to hand test and strength comparison data published by Holler and Fromm (2010), based on guidelines within the International Classification of Seasonal Snow on the Ground (Colbeck et al., 1990) (different to that within the International Classification of Seasonal Snow on the Ground (Fierz et al., 2009)). Note that axes are rotated by 90 degrees.

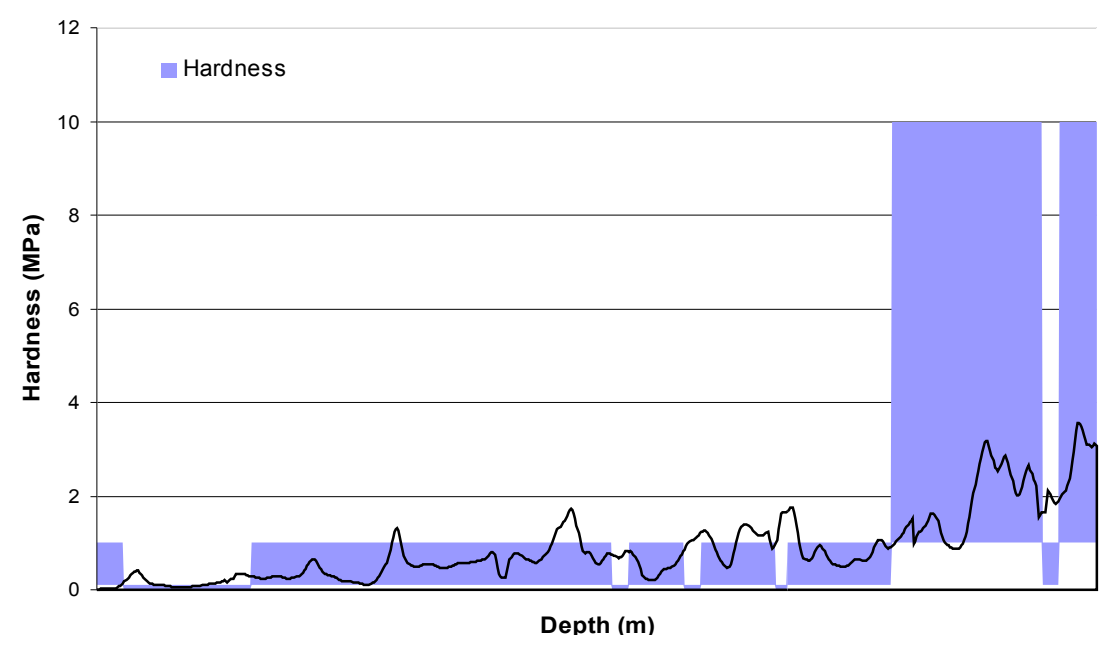

Figure 7.9: Comparison of CPT tip resistance (Test 9) with quantified hand hardness range from snow pit \# 1. Hardness range is shown as blue bars.

This comparison suggests that tip resistance is related to hand hardness, although discrepancies in both alignment and magnitude are apparent. Discrepancies in alignment are probably because of misidentification or interpretation of hardness layers, and peaks in resistance are obvious where no harder layers were discerned, confirming that hardness measured by CPT is a much higher resolution parameter than hardness measured via a subjective manual method. Discrepancies in magnitude are further considered below.

Table 7.1 compares tip resistance values averaged over hardness layers with order of magnitude strengths (Colbeck et al., 1990) utilised to generate Figure 7.9.

This tabled data suggests that average resistance values obtained via CPT are routinely greater than predicted strengths (from hardness), and that hand hardness values appear unable to resolve the data to the degree demonstrated by the CPT. Table 7.2 compares hand hardness, Rammsonde Number and CPT Force measurements (CPT tip resistance converted to force using standard cone cross-sectional area):

These values are consistent with the previous table and suggest that resistance measured by cone penetrometer is perhaps twice as great as resistances expected via 
Table 7.1: Comparison between hand hardness and CPT hardness (CPT conducted at a penetration rate of $25 \mathrm{~mm} \mathrm{~s}^{-1}$ ).

\begin{tabular}{|c|c|c|c|c|c|c|}
\hline Object & Index & $\begin{array}{l}\text { Lower } \\
\text { (MPa) }\end{array}$ & Limit & $\begin{array}{l}\text { Upper } \\
\text { (MPa) }\end{array}$ & Limit & $\begin{array}{ll}\text { CPT } & \text { Hardness } \\
(\text { Test 9) } & (\mathrm{MPa})\end{array}$ \\
\hline 1 & fist & 0 & & 0.001 & & $\mathrm{~N} / \mathrm{A}$ \\
\hline 2 & 4 fingers & 0.001 & & 0.01 & & $\mathrm{~N} / \mathrm{A}$ \\
\hline 3 & 1 finger & 0.01 & & 0.1 & & 0.66 \\
\hline 4 & pencil & 0.1 & & 1.0 & & 1.25 \\
\hline 5 & knife blade & 1.0 & & - & & 2.68 \\
\hline 6 & ice & $\mathrm{N} / \mathrm{A}$ & & $\mathrm{N} / \mathrm{A}$ & & 5.6 \\
\hline
\end{tabular}

Table 7.2: Force comparison between hand hardness, Rammsonde Number and CPT average tip force (CPT conducted at a penetration rate of $25 \mathrm{~mm} \mathrm{~s}^{-1}$ ).

$\begin{array}{clll}\text { Object } & \text { Index } & \begin{array}{l}\text { Ramm. Num- } \\ \text { ber Range }\end{array} & \begin{array}{l}\text { Average Tip } \\ \text { Force }(\mathrm{N})\end{array} \\ 1 & \text { fist } & 0-50 & \text { N/A } \\ 2 & \text { 4 fingers } & 50-175 & \text { N/A } \\ 3 & \text { 1 finger } & 175-390 & 600 \\ 4 & \text { pencil } & 390-715 & 1200 \\ 5 & \text { knife blade } & 715-1200 & 2600 \\ 6 & \text { ice } & >1200 & 5500\end{array}$

Rammsonde; such differences between similar measures of resistance are intriguing. Pielmeier and Schneebeli (2003) in comparing SMPT and ram hardness values note that the relationship between the two is not linear, but exponential, and a similar relationship can be seen between CPT tip resistance data and Rammsonde numbers (Figure 7.10).

Pielmeier and Schneebeli (2003) suggest that this exponential relationship is because in the extremely hard layers they tested (ram hardness $>500 \mathrm{~N}$ ), the SMPT can detect thin hard layers (SMPT has resolution of $\sim 1 \mathrm{~mm}$ ) which the Rammsonde (resolution $\sim 30 \mathrm{~mm}$ ) can't detect. Pielmeier and Schneebeli (2003) also note the disparity between SMPT and hand hardness readings in hard snow, where again SMPT readings are exponentially greater than hand hardness readings at ram hardness values $>500 \mathrm{~N}$. The CPT though is of similar dimension to the Rammsonde, so why should it display characteristics consistent with the smaller SMPT?

Factors which may describe why hardness measured via different instruments varies include:

1. differences in penetration rate and thus strain rate,

2. variation in insitu vertical stress,

3. differences in penetrometer size,

4. observer objectiveness (for hand hardness measurements), and 


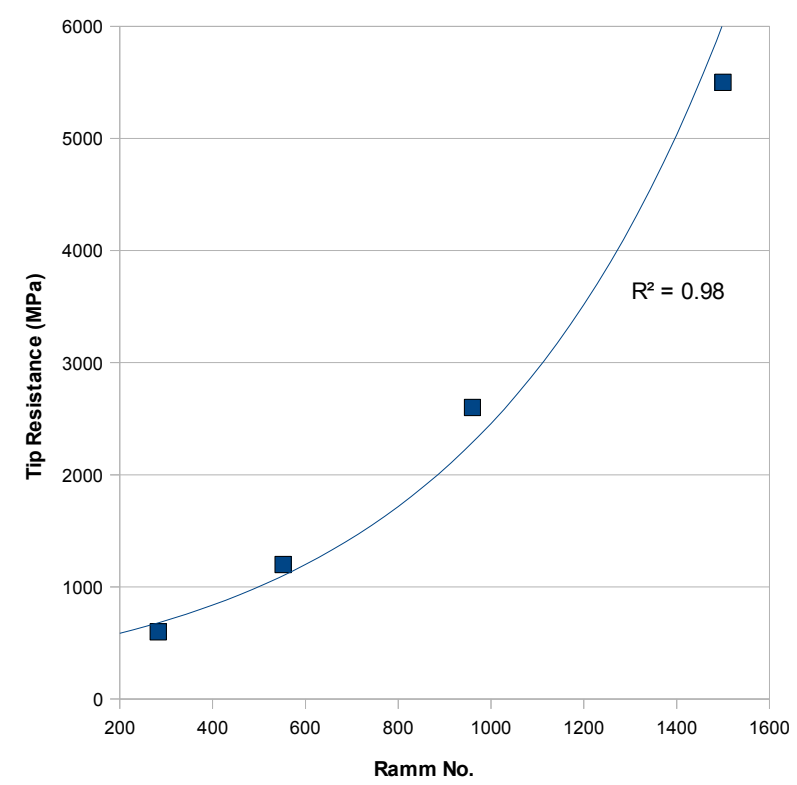

Figure 7.10: Comparison between cone tip resistance and Ramm number shows an exponential relationship - consistent with the relationship between Ramm number and SMPT data.

5. method of testing.

The Rammsonde is hammered into the snow in a non-continuous manner. As it contacts the snow it decelerates hence discrepancies owing to a rate-effect might be expected. If typical penetrative speeds (actual speeds are not known to have been measured) are generally greater than the standard $20 \mathrm{~mm} \mathrm{~s}^{-1}$ used for CPT then decreased resistance values might be expected.

CPT and the SMPT test are conducted at similar speeds using similarly shaped cones, the obvious difference between the tests being penetrometer size. A size effect has previously been discussed and the larger size of the CPT cone implies smaller resistances than those measured by SMPT. Also, measured resistance via CPT might be less than actual layer resistance, because of the size of the cone and its inability to obtain a steady-state resistance value within thin layers; layers in which resistance may be more accurately determined via a smaller instrument such as the SMPT.

Variation in hardness measured by SMPT, Rammsonde and CPT has been observed, and numerous factors affecting this variation have been noted. Such variation is not inconsistent with other published data (Pielmeier and Schneebeli, 2003), and direct comparative testing between instruments would be of interest to further elucidate these differences. Sleeve friction measurements are not expected to provide a useful proxy for snow hardness as friction is measured in post-fractured, non-virginal material.

The primary aim of this thesis is discussed next: Can snow strength be estimated from CPT? 


\subsection{Snow strength estimation}

In this section my understanding of snow strength from CPT is summarised and then a number of existing methods for deducing snow strength from penetrative data are examined, to see whether they are applicable for CPT. A new physical model deriving shear strength from CPT is then presented, and results are shown to be consistent with other representative snow strength data collected at Halley.

The CPT measures snow resistance or hardness. Reiterating, hardness is a measure of semi-confined strength in compression; semi-confined because although no external confinement is applied, confinement due to the insitu nature of the testing exists, and in compression because that is the nature of the testing method, even though failure of the material on a microstructural level may occur via numerous means, including shear, bending, compression, tension etc.

Before examining existing methods of deducing snow strength and then elucidating a relationship between CPT tip resistance and strength, the factors which may influence CPT tip resistance are briefly outlined again to summarise their impact on layer strength assessment:

1. Effective Area. The effective area of the cone will likely be greater than the actual cone cross-sectional area (by $\sim 7 \%$ ) thus resulting in potential over-reading of actual resistance;

2. Penetration Rate. Significant rate effects have been observed, hence any derived resistance/strength is pertinent to that rate of testing only, noting also that (immeasurable) variations in strain-rate at the cone tip are probably occurring as snow parameters (density, microstructure etc.) vary, even at a constant penetration rate;

3. Cone shape. The shape of the cone will affect whether a compacted zone forms ahead of the cone (which will require realignment of the depth scale and probable reconsideration of the physical processes at work.) No compacted zone was observed to form ahead of the $60^{\circ}$ cone used in this testing. However, in alpine snow, moister snow or during testing with a blunter cone, such a compacted zone may form;

4. Cone size. Size effects were observed and must be considered if comparing data from cones of different size; and

5. Overburden pressure. Although increased confinement was expected to have an effect on tip resistance, observed differences were not statistically significant, hence at this time, no additional modification is made.

Review of these effects suggests that in estimating layer strength from a CPT trace: 
1. some reduction in measured cone resistance may be appropriate owing to the effective area, and

2. any strength derived is at stated penetration rate, cone size and shape, in a defined snow type.

Additional considerations which complicate the assignment of a quantitative strength measure to a snow layer from CPT data include:

1. ambiguity in determining the beginning and end of layers within the snow pack because of the cone's 'critical depth': Where does the assigned strength start?,

2. likely under-reading in layers thinner than the cone critical depth (assumed $\sim$ $25 \mathrm{~mm}$ ); calculations based on Lunne et al.'s discussion (Lunne et al., 1997) on Vreugdenhil et al.'s method (Vreugdenhil et al., 1994) of adjusting tip resistance values if the critical depth is not met suggest that actual cone tip resistances may be more than double measured cone tip resistance, and

3. probable over-reading in lower density snow, consistent with Gibson and Ashby's observation (Gibson and Ashby, 1997) that the compressive strength of highporosity foam increases significantly when failed fragments fill the pore spaces, as might be expected in higher porosity snow.

Incorporation of the friction sleeve is a unique asset of the CPT. However, consideration thus far suggests that because friction is measuring the snow in a post-fractured state, frictional information is not representative of virgin snow properties. It was speculated earlier that sleeve friction data may provide guidance on snow microstructure, a determinant of snow strength, but based on the work conducted thus far, it is not believed that friction data can contribute usefully to the derivation of strength from CPT.

\subsubsection{Existing methods for deducing snow strength}

In this section a number of existing methods for deducing snow strength from penetrative instruments are examined in an attempt to assess their applicability to CPT.

Perhaps the most complete conceptualisation of the possible mechanics ahead of an advancing cone in granular material is Johnson's model (Johnson, 2003), which has been noted throughout this work. Johnson's work is an amplification of earlier work, notably Schneebeli et al. (1999), and it has been further refined and examined by numerous authors including Marshall (2005) and Floyer (2008). The viability of applying this model to my CPT work is briefly examined here. Johnson's model is not a uniquely physical conceptualisation but a statistical micromechanical model of the form: 


$$
\text { Max Resistance }=N P F_{a v}
$$

where $N$ is the number of microstructural elements (grains / grain bonds) available to contact the penetrometer (or the larger Penetrometer Effective Surface (PES) surrounding the cone), $P$ is the probability that each of these elements is in contact with the penetrometer (and is thus contributing to measured resistance), and $F_{a v}$ is the average force that each element (fractured or whole) contributes. This average force equals half the maximum resistance at brittle fracture, where the maximum resistance depends on maximum deflection of a microstructural 'element'.

Johnson (2003) considers the sum of microstructural elements acting on the cone (or PES), their strength, orientation etc. and their probability of contributing to resistance, all applied to the initial brittle fracture of the material, but not incorporating the post fracture compaction which is expected to contribute markedly to measured tip resistances. However, this compaction is included in Johnson's calculation of probability, and increased compaction will probably result in increased number of elements in contact with the cone/PES.

After deriving additional equations to estimate $N, P$ and $F_{a v}$ Johnson combines these to derive a physical expression for maximum penetrative force; this equation and his nomenclature are presented at Appendix C, along with representative values. Although Johnson's model has been shown to generate results consistent with SMPT data (Marshall and Johnson, 2009), accurate application of Johnson's method to CPT is not easily achievable, primarily because of the large number of microstructural variables necessary to enable calculation. Other existing methods of estimating snow strength from CPT are now assessed for their applicability, and then a physical model is proposed.

A classical method of assessing the strength or hardness of snow is via the Rammsonde equation developed by Haefeli (1936) in conjunction with the Rammsonde tool. This standard equation attempts to estimate the work (force times distance) done in penetration in an attempt to characterise the hardness of the snow, and is repeated below:

$$
\bar{R}=\frac{E}{S}=\frac{1}{S} \cdot\left[M_{H} \cdot g \cdot h+\left(M_{H}+M_{S}+M_{G}\right) \cdot g \cdot s\right]
$$

$\bar{R}$ is mean ram hardness, $S$ is penetration distance, $g$ is gravitational acceleration, $h$ is height of hammer fall, $M_{H}$ is mass of hammer, $M_{G}$ is mass of hammerguide, $M_{S}$ is mass of shaft including probe and $E$ is total energy transferred to the probe. Irwin et al. (1991) compared the Rammsonde and a cone (not a standard cone penetrometer) using a similar equation, yet no gain is made in applying this technique to CPT as the resultant force / area is the same as the measure of resistance obtained directly from the CPT. CPT tip resistance is a direct measure of work (average force measured 
during $5 \mathrm{~mm}$ penetration divided by cone area), hence no further consideration of work analogies is necessary. This discussion serves to clarify that work (Joules) can readily be derived directly from CPT data should such units prove necessary (say for comparison with work done in disaggregation of snow of a certain density and bonding) and that direct comparison between data derived via CPT and historical Rammsonde data is also valid (noting the inherent inaccuracies with Rammsonde data owing to variations in penetration speed, size / shape etc.).

In soils, cone penetration resistance is typically interpreted via five methods: bearing capacity, where the cone resistance is assumed to be equal to the collapse load of a deep circular foundation; cavity expansion, where a relationship is assumed between cone resistance and the pressure required to expand a spherical cavity in the material from an initial radius of zero; steady-state deformation, where the penetration process is viewed as a steady state flow of material past a fixed penetrometer; incremental finite-element analysis, where incremental plastic loading is applied, and the collapse load is assumed to be equal to the cone tip resistance, or calibration chamber testing, where large calibration chambers have been used to establish empirical correlations between cone resistance and material properties ( $\mathrm{Yu}$ and Mitchell, 1998). Arbitrary Lagrangian-Eulerian (ALE) analysis, where the benefits of both Lagrangian (movable mesh nodes) and Eulerian (fixed mesh nodes) numerical analysis are combined, has also recently been adopted ( $\mathrm{Lu}$ et al., 2004). Direct application of these methods to snow is not trivial, primarily because of the extreme brittleness and compressibility of snow when subjected to high penetration rates.

One steady-state method is the strain path method, based on the presumption that the penetration of a cone can be viewed as a steady-state flow of homogeneous material past a static penetrometer (Teh et al., 2008). A flow pattern and thus the strain path for each element is estimated, and constitutive equations for the flow are applied, thus generating deviatoric stresses; Floyer and Jamieson (2006) applied this technique empirically to determine the strain paths around a penetrometer. Similarly, Particle Image Velocimetry (PIV) is increasingly used to observe and record particle movement in snow (Gleason, 2004). Although valuable empirical research has occurred, theoretical application of steady-state methods is not readily applicable to snow because of its highly compressible nature and the difficulty in defining constitutive equations.

Ladanyi (1985) devised techniques using constant stress tests (rather than constant rate tests) to relate cone resistance at different rates with different size penetrometers or piles in frozen soils, and he provided calculations for deriving cohesion and thus shear strength from these CPT data. Unfortunately Ladanyi's methods rely on plastic deformation and do not consider compaction, hence they are not readily transferable to low to medium density snow. Application of such methods may be feasible if final strains and stresses post-compaction are further analysed, possibly through application of the cavity expansion method, a method which appears under-utilised in the 
examination of snow behaviour during penetration.

I investigated cavity expansion methods extensively during the preliminary phases of my research. However, such methods are only useful in investigating post-fracture compactive behaviour and are not able to encapsulate the entire penetrative process incorporating elastic deflection, brittle fracture and then work hardening due to compaction; also, most methods cannot incorporate volume straining and assume elastic perfectly-plastic behaviour. So, cavity expansion methods remain valuable for examining facets of snow's behaviour during penetration, yet at this time they cannot be used in isolation to predict ultimate cone tip resistance in snow.

To summarise, a number of existing methods used to derive strength from penetrative data suggest that no suitable method currently exists to adequately predict snow strength from CPT. In order to address this deficiency a conceptual physical model derived from data obtained at Halley is presented below.

\subsubsection{Conceptual Evaluation}

This section attempts to describe the behaviour of polar snow undergoing cone penetration in an effort to estimate snow strength from tip resistance. Johnson (2003) in his model on penetration in granular materials provides a very good basis for understanding the likely processes occurring ahead of a penetrating cone. I outlined my qualitative description of this behaviour in Chapter 2 and it is summarised very simply below. As the cone is driven through an (assumed) bonded matrix of snow, two things must occur to allow its progress:

1. firstly, the bonds linking grains within the snow must be broken, then

2. the fractured material must be displaced and compacted into the snow surrounding the cone.

That is, first the cohesion of the snow is overcome and then a frictional process will occur as fragments are compacted under (some unknown) confinement, a process I consider analogous to semi-confined compression testing of snow. In the case of the standard $36.7 \mathrm{~mm}, 60^{\circ}$ cone, compaction will occur out to a distance of $\sim 21 \mathrm{~mm}$, the maximum cone radius normal to the cone face (see Figure 7.11), as fractured particles are forced out normally from the cone tip eventually into the hole wall at the cone shoulder.

Fractured particles will be displaced normal to the cone face (Johnson, 2003). However, Gill (1968) clarify that "due to the soil-tool friction, the direction of the resultant force is inclined forward of the normal by an angle $\delta$ ". This interaction is ignored for the moment because the frictional effect is an order of magnitude less than the normal forces acting on the cone. 


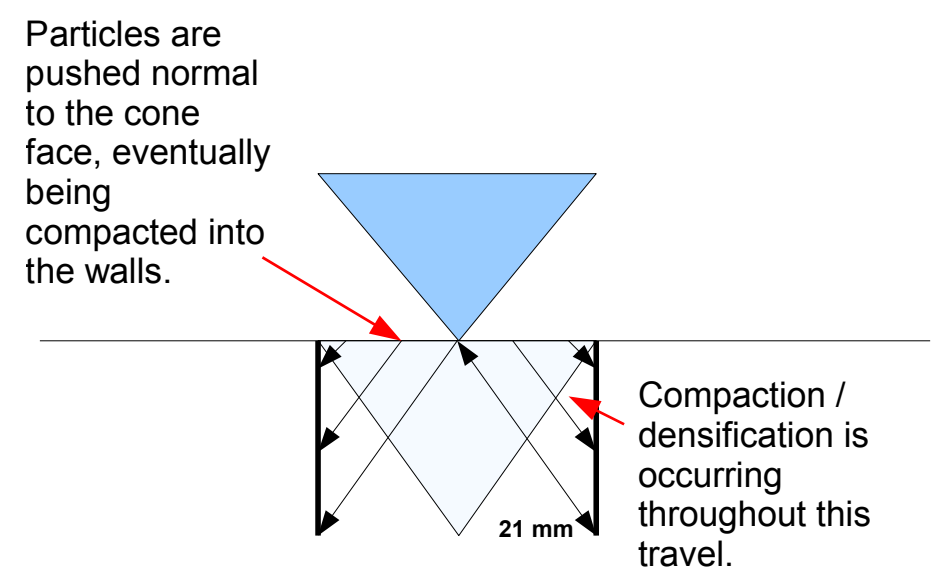

Figure 7.11: Possible strain path for fractured particles moving ahead of cone.

I propose an equation of the form below to describe the sum of stresses measured by the cone tip during penetration:

$$
q_{c}=C_{\text {vert }}+S_{\text {vert }}+F_{\text {vert }}
$$

where $q_{c}$ is total tip resistance, $C_{v e r t}$ is the vertical component of cohesion (assumed equivalent to shear strength), $S_{\text {vert }}$ is the vertical component of the stress required to compact the snow (analogous to semi-confined compression testing), and $F_{\text {vert }}$ is the small frictional stress between the snow and the cone face (vertical component). In a later section a rearranged version of this equation is presented from which snow shear strength can be estimated from CPT tip resistance.

One point that needs to be clarified is that the cohesion value in equation 7.4 is a measure of snow shear strength, whereas resistance tests such as CPT have previously been shown to be a measure of strength in compression. It was previously shown that compressive strength is equivalent to twice the shear strength hence any strength values derived for equation 7.4 from shear data will need to be multiplied by two to arrive at an estimate for tip resistance (strength in compression). Each term in Equation 7.3 is now discussed.

\subsubsection{The cohesion term (C)}

The cohesion component within Equation 7.4 is provided by the ice bonds formed between ice grains via sintering. I consider this equivalent to the shear strength of the snow prior to fracture. Representative values for snow of various densities can be obtained from sources such as Mellor (1975) and Shapiro et al. (1997). A value for cohesion may also be estimated by considering toughness.

McClung (1981) says that the most important property with regards to failure within the snow slab is shear fracture toughness, and that shear strength is its most important component; other influencing factors include stiffness, macroscopic imper- 
fections and variations in the energy to generate fracture surfaces. Thus, fracture toughness and cohesion should be analogous.

Fracture toughness $\left(K_{I C}\right)$ is a material property which denotes the critical stress intensity factor for the material at hand (Schulson and Duval, 2009). It is based upon the theory of linear-elastic-fracture mechanics (LEFM) conceived by Griffith (1921). The IC subscript denotes the ability of the assumed-cracked material to resist fracture via tension normal to the assumed crack. Once $K_{I C}$ is surpassed, crack propagation, ultimately to failure will occur. Although crack propagation can occur in tension (Type I), shear (Type II) or tearing (Type III), the predominant mode of failure in most materials, including ice, is tearing (Schulson and Duval, 2009) hence it is assumed that $K_{I C}$ is a representative fracture toughness for a macro sample, even though crack propagation may occur via all three mechanisms. Schulson and Duval (2009) state that fracture toughness in shear is of similar value to fracture toughness in tension.

Schulson and Duval (2009) present the following equation for fracture toughness for snow up to a density of $\sim 460 \mathrm{~kg} \mathrm{~m}^{-3}$ :

$$
K_{I C}=A\left(\frac{\rho_{\text {snow }}}{\rho_{\text {ice }}}\right)^{S}
$$

where the coefficient $\mathrm{A}\left(\mathrm{kPa} \mathrm{m}^{\frac{1}{2}}\right)$ and the exponent $S$ are determined experimentally. These parameters range from $\mathrm{A}=7.8 \mathrm{kPa} \mathrm{m}^{\frac{1}{2}}$ and $S=2.3$ for very warm snow at $-1^{\circ} \mathrm{C}$ (Kirchner, 2001) to $\mathrm{A}=21.6 \mathrm{kPa} \mathrm{m}^{\frac{1}{2}}$ and $\mathrm{s}=2.1$ for colder snow at $\sim-10^{\circ} \mathrm{C}$ (Schweizer et al., 2004). If the numbers obtained at $-10^{\circ} \mathrm{C}$ are used then a value for $K_{I C}$ of $\sim 5 \mathrm{kPa} \mathrm{m}^{\frac{1}{2}}$ is obtained, for snow of density $450 \mathrm{~kg} \mathrm{~m}^{-3}$. This lies within the range for shear fracture toughness estimated by McClung (2005) of between 0.02 and $13 \mathrm{kPa} \mathrm{m}^{\frac{1}{2}}$.

A second equation from Schulson and Duval (2009) can then be considered to relate tensile strength and fracture toughness within ice polycrystals:

$$
\sigma_{t}=\frac{K_{I C}}{\lambda^{\frac{1}{2}}} d^{-\frac{1}{2}}
$$

where $\sigma_{t}$ is tensile strength, $\lambda$ is an experimentally determined coefficient $(\lambda=3.7)$ and $\mathrm{d}$ is grain size $(\mathrm{mm})$. Applying this equation for an average observed grain size of $0.75 \mathrm{~mm}$ and a calculated $K_{I C}$ of $\sim 5 \mathrm{kPa} \mathrm{m}^{\frac{1}{2}}$ results in an estimated tensile strength for the snow of $95 \mathrm{kPa}$. The tensile strength of snow is of the same order as shear strength (Shapiro et al., 1997) so this value for tensile strength can also be assumed representative of shear strength. It lies within the range of shear strength values $(\sim$ $70-115 \mathrm{kPa}$ ) presented by Mellor (1975) for snow of this density.

The above derivation of cohesion via fracture toughness relies on the assumption that crack propagation within open cellular snow $\left(\rho<\sim 830 \mathrm{~kg} \mathrm{~m}^{-3}\right)$ occurs in a similar manner to propagation within polycrystal ice. This assumption is somewhat precarious as the crack length is of similar dimension to the grain size, and the applicability of 
LEFM depends on the structure size (Sigrist, 2006). However, Petrovic (2003) shows that the snow fracture flaw diameter (for snow of density $\sim 400 \mathrm{~kg} \mathrm{~m}^{-3}$ ) typically equals $\sim 0.2 \mathrm{~mm}$, so use of this method may remain warranted. The cohesion values derived via this method appear realistic, and in snow at depth (or in processed snow, such as trafficked layers at Halley) where increased sintering and compaction has occurred and the snow approaches the nature of a 'porous ice' rather than a 'bonded matrix', then estimates for cohesion obtained via this method may become increasingly valid.

Measured tip resistance will be greater than the actual material strength owing to the additional resistance component due to compaction, thus we now turn our attention to the second component of equation $7.4, S_{\text {vert }}$.

\subsubsection{The compactive term (S)}

Initial inspection suggests that the compactive component might be estimated in a number of ways, including examination of:

1. empirical data, relating pressure and strain rate in naturally densifying snow, or

2. by using a theoretical model of some form.

However, closer inspection suggests that application of empirical data, say from Mellor (1975) is not appropriate for the rates and pressures considered here which are far greater than those experienced in the natural densification of surface snow, primarily via creep processes, hence this concept is not considered further.

A compactive model is used by Johnson (2003) to describe the amount of compaction available within a particulate material during penetration. Johnson defines a compaction coefficient $\beta$, that relates the volumetric strain in compaction with the change in density of the material relative to its initial density:

$$
\beta=\frac{1-\rho_{o}}{\rho}
$$

where $\rho$ is snow density during compaction $\left(\mathrm{kg} \mathrm{m}^{-3}\right)$ and $\rho_{o}$ is initial snow density. A critical compaction coefficient $\beta_{c r}$ can also be defined for the critical material density $\rho_{c r}$, which is the "critical material density when a granular material locks up and further compaction through particle rearrangement no longer occurs" (Johnson, 2003):

$$
\beta_{c r}=\frac{1-\rho_{o}}{\rho_{c r}}
$$

$\beta$ can also be defined in terms of radii relative to the tip of a penetrating cone:

$$
\beta=\left(\frac{r_{i}}{r_{f}}\right)^{2}
$$

where $r_{i}$ is the initial pre-compaction radius from the cone and $r_{f}$ is the final radius $(21 \mathrm{~mm})$ post-compaction, defined normal to the face of a penetrometer from its tip (see 
Figure 7.11). Equations 7.8 and 7.9 can be used to apply conservation of mass across a change in volume normal to the face of a penetrating cone in a granular material; $r_{i}$ and $r_{f}$ can be estimated, $\beta$ can be obtained and then final density after compaction can be determined.

Table 7.3 applies this theory to postulated values for a general particulate material, based on a $36.7 \mathrm{~mm}$ cone diameter, assuming that compaction is occurring normal to the penetrometer face (neglecting minor changes in this angle because of friction (Gill, 1968)):

Table 7.3: Derivation of 'lock-up' density for hypothetical initial density and radii values of a general particulate material.

$\begin{array}{lcccc}\left.\begin{array}{l}\text { Initial density } \\ (\mathbf{k g ~ m}\end{array} \mathbf{m}^{-3}\right) & r_{i}(\mathbf{m m}) & r_{f}(\mathbf{m m}) & \beta & \rho_{c r}\left(\mathbf{k g ~ m} \mathbf{~ m}^{-3}\right) \\ 450 & 1 & 21 & 0.0023 & 451 \\ 450 & 5 & 21 & 0.0567 & 477 \\ 450 & 10 & 21 & 0.2268 & 582 \\ 450 & 15 & 21 & 0.5102 & 919 \\ 450 & 17 & 21 & 0.6553 & 1305\end{array}$

The results from this table suggest:

1. if lock-up occurs earlier, ie when $\rho_{c r}$ is less, then penetrometer resistance will be greater,

2. for $\rho_{c r}$ to be less, a lower $\beta_{c r}$ is needed, and

3. $\beta_{c r}$ is less when $\Delta \mathrm{r}\left(r_{f}-r_{i}\right)$ is greatest.

So when $\Delta \mathrm{r}$ is greater, $\rho_{c r}$ is less, 'lock-up' occurs earlier and resistance increases, or, as $\rho_{o}$ increases, possible compaction is decreased, and therefore the normal force on the cone, affecting both tip resistance and sleeve friction, is expected to increase.

For snow, maximum packing occurs at a relative density of $\sim 0.6$ or a density of $\sim 550 \mathrm{~kg} \mathrm{~m}^{-3}$. If $\rho_{o}$ equals $450 \mathrm{~kg} \mathrm{~m}^{-3}$ then $\beta_{c r}$ equals 0.1818 . Therefore ${ }^{r_{i}} / r_{f}$ equals 0.426 and $r_{i}$ equals $\sim 9 \mathrm{~mm}$ (assuming $r_{f}$ equals $\sim 21 \mathrm{~mm}$ ). This means that maximum compaction (to $\rho=\sim 550 \mathrm{~kg} \mathrm{~m}^{-3}$ ) will occur after normal displacement out to $9 \mathrm{~mm}$.

The increase in stress as compaction occurs to the maximum packing density of $\sim$ $550 \mathrm{~kg} \mathrm{~m}^{-3}$ can possibly be examined by using the Quasi-plastic Volumetric Modulus (K) for snow (Mellor, 1975), where a volumetric strain of 0.43 requires a stress increase of $\sim 8.6 \mathrm{kPa}$ (using $\mathrm{K} \sim 20 \mathrm{kPa}$ from Mellor's published data). But, to assess the stress increase beyond the maximum packing density, application of Mellor's Quasiplastic Volumetric Modulus is less appropriate as it pertains to the description of naturally loaded snow undergoing slow deformation. For medium density snow, Mellor suggests that strain rate "may be proportional to the fourth power of stress" therefore an alternative method of relating stress to volumetric strain is required. 
Because specific volume is the reciprocal of density, any graph describing a systematic relationship between pressure and density can be interpreted as an equation of state. Therefore, for densities and rates greater than those represented by the Quasiplastic Volumetric Modulus, a measure of stress increase can be obtained by direct examination of published stress/density curves, such as Figure 7.12 taken from Mellor (1975). This relationship provides the basis for estimating the large stress increases expected owing to compaction beyond the maximum packing density.

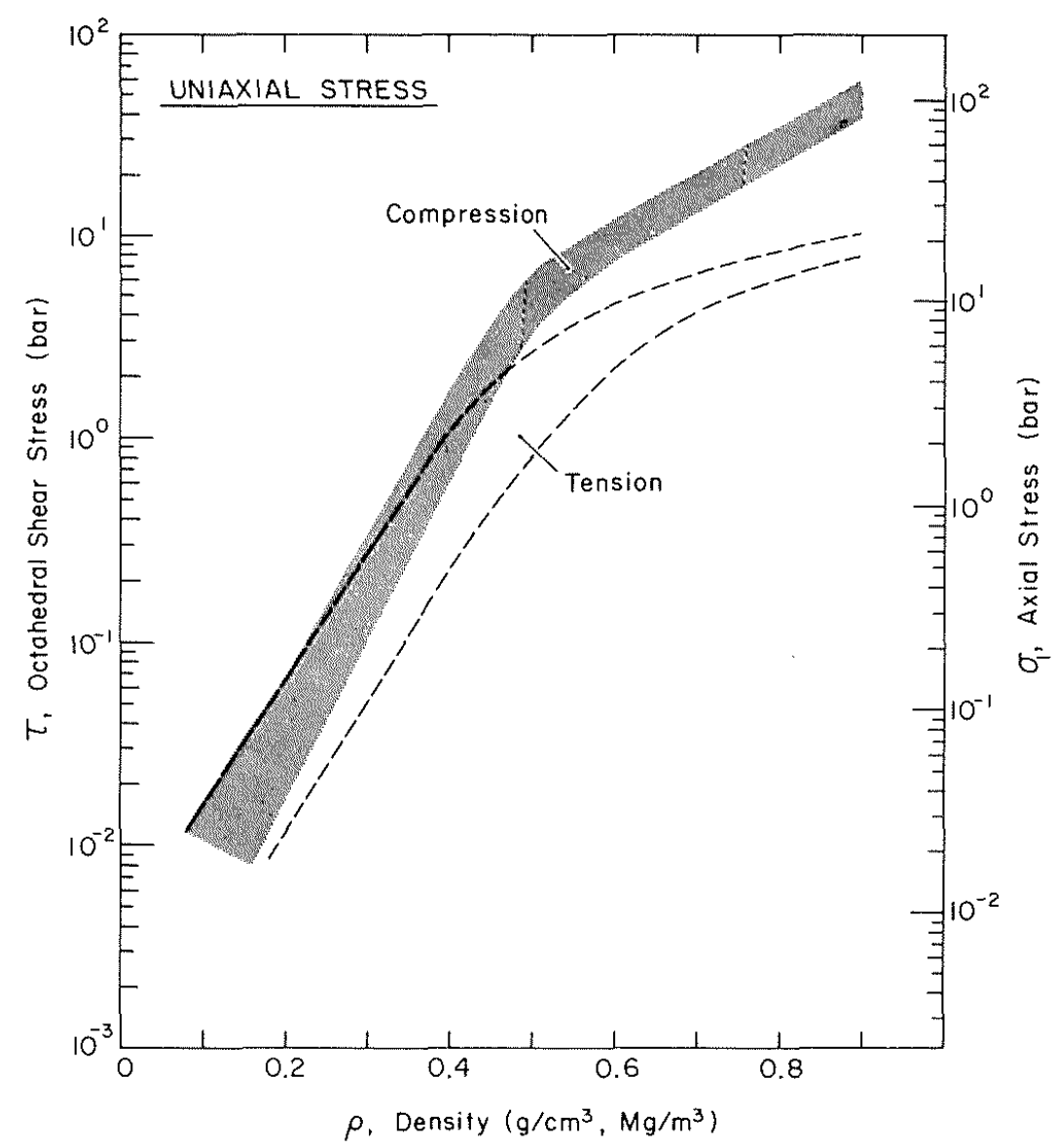

Figure 7.12: "A systematic relationship between pressure and density can be interpreted as an equation of state" (Mellor, 1975). This and similar graphs from Mellor (1975) provide the basis for estimating stress increases due to compaction beyond the maximum packing density.

Estimation of the increase in stress out to the final radius of $\sim 21 \mathrm{~mm}$ can now be made using pressure / density data from Mellor (1975). But, the problem with deducing a stress increase via such volumetric compaction is that the deduced value depends on two interdependent unknowns: the final density after compaction, and compacted volume. Final density after compaction was not measured, so some other way of estimating compacted volume post CPT is necessary.

One observation was made that can be used to define this post-CPT compacted volume. After Test 9, a portion of the snow surrounding the CPT hole was excised and the annulus thickness was measured to be $\sim 2.2 \mathrm{~mm}$ (see Figure 5.22), for snow 
of initial density $\sim 450 \mathrm{~kg} \mathrm{~m}^{-3}$. This value can be used to define the final compacted volume, so then a final density, and the stress required to effect this compaction can be obtained (via Mellor (1975)) as per Table 7.4. A unitless pressure multiplier can be obtained by the following equation:

$$
M=\frac{\sigma_{1\left(\rho_{\text {fin }}\right)}}{\sigma_{1\left(\rho_{\text {init }}\right)}}
$$

where $\sigma_{1 \rho_{\text {fin }}}$ is the uniaxial compressive strength at the final density and $\sigma_{1 \rho_{\text {init }}}$ is the uniaxial compressive strength at the initial density.

Table 7.4: Increase in stress required to compact snow from initial to final density.

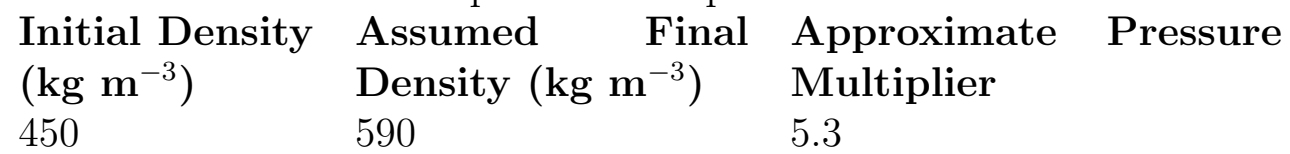

This observation is consistent with work by Kartashov (1965) on the mechanical properties of snow and firn. He found via plate-testing that increasing the density of snow from $\sim 450 \mathrm{~kg} \mathrm{~m}^{-3}$ to $\sim 590 \mathrm{~kg} \mathrm{~m}^{-3}$ required an approximately five-fold increase in pressure. This method provides a means of estimating the stress increase across the whole range of volumetric compaction occurring at the cone tip during CPT. Rate dependency of this multiplier is not considered herein. Mellor (1975) makes no mention of such, Johnson (2003) does not consider rate effect on compactive stresses and compressive testing of particulates (sands) at varying rates suggests limited rate effect on stress (Lunne et al., 1997).

The stress required to effect compaction at the cone tip will vary with initial density. Abele's work (Abele, 1970) on the penetration of rigid plates into snow confirms that at the same penetration distance, the stress increase is greatest in higher density snow, and similarly for the same applied pressure, settlement will decrease as initial density increases. This work was further verified by Abele and Gow (1976) in high density snow at higher penetration rates. Table 7.5 summarises the variation in strain with density derived from this testing (Abele and Gow, 1976).

Table 7.5: Variation of linear strain with initial density at constant stress (derived from (Abele, 1970)).

$\begin{array}{cc}\text { Density }\left(\mathrm{kg} \mathrm{m}^{-3}\right) & \text { Strain (\%) } \\ 400 & 0.48 \\ 450 & 0.415 \\ 500 & 0.35 \\ 550 & 0.28 \\ 600 & 0.22 \\ 650 & 0.16\end{array}$

Scapozza and Bartelt (2003b) in triaxial tests on snow show that volumetric strain is similar in magnitude to linear strain for snow of initial density $270 \mathrm{~kg} \mathrm{~m}^{-3}$ at various 
confining pressures, so it is assumed that values for volumetric strain are similar to those values for linear axial strain presented in Table 7.5. This relationship is also assumed to hold across a range of applied stresses, consistent with data presented within Abele and Gow (1976).

Therefore, the stress increase required for densification across a range of densities can be estimated from Mellor (1975) and stress multipliers for each initial density can be generated. These estimates are shown in Table 7.6:

Table 7.6: Stress multiplier derived from assumed initial and final density and pseudoconstant post-test wall thickness of $\sim 2.2 \mathrm{~mm}$; see Figure 5.22.

$\begin{array}{llll}\text { Initial Density } & \begin{array}{l}\text { Volumetric } \\ \left(\mathbf{k g ~ m} \mathbf{~ m}^{-3}\right)\end{array} & \begin{array}{l}\text { Assumed Final } \\ \text { Strain }\end{array} & \begin{array}{l}\text { Density }\left(\mathrm{kg} \mathrm{m}^{-3}\right) \\ \text { Approximate Pres- } \\ \text { sure Multiplier (M) }\end{array} \\ 400 & 0.28 & 555 & 3.7 \\ 450 & 0.24 & 590 & 5.3 \\ 500 & 0.2 & 625 & 7.2 \\ 550 & 0.16 & 660 & 9.2 \\ 600 & 0.13 & 690 & 11.1 \\ 650 & 0.09 & 720 & 11.6\end{array}$

This table shows that as initial density increases, volumetric strain decreases, change in density decreases and the pressure to produce volume change increases, as suggested by an increased multiplier. These trends are consistent with Abele (1970), Abele and Gow (1976) and Kartashov (1965) and may suggest how the pressure multiplying factor varies at different initial snow densities. Fitting the data in Table 7.6 allows $\mathrm{M}$ to be estimated for any density using:

$$
M=\left(0.0335 \rho_{\text {initial }}\right)-9.5828 \text { for } 350<\rho_{\text {initial }}<650 \mathrm{~kg} \mathrm{~m}^{-3}
$$

These estimates can be used to estimate the second term $(\mathrm{S})$ of Equation 7.3 to derive shear strength from tip resistance in snow of different initial density. This second term is purely a multiple of the first term $\mathrm{C}$ and the estimated multiplier $(\mathrm{M})$ obtained from Table 7.4 (or Equation 7.11). The second term (S) is therefore of the form $\mathrm{Mx}$ C.

One final term in Equation 7.3 needs to be addressed: the third term F, due to the surface friction between compacted snow particles and the surface of the cone.

\subsubsection{The cone-face friction term $(\mathrm{F})$}

The cone-face friction term $(\mathrm{F})$ is merely the frictional force on the cone face due to the normal force generated during compaction. A coefficient of friction for snow of $\mu=$ $\sim 0.1$ is representative of values presented by both Bowden (1953) and Colbeck (1988). A suitable value for $\mathrm{F}$ is therefore obtained merely by multiplying the second term in equation $7.3(\mathrm{~S})$ by 0.1 . 
Quantification and verification of these terms will shortly occur but firstly two additional modifying factors noted earlier in this chapter, rate and effective size are considered.

\subsubsection{Modifying factors}

Because CPT is conducted at rates greater than that from which strength data within Mellor (1975) are obtained, resistance via CPT may be $\sim 20 \%$ less, as suggested by Figure 5.15. So, strength values from Mellor (1975) should be reduced by $20 \%$ before incorporation into Equation 7.3. This reduction will only affect the first term (C) and not the multiplier (M) which will remain constant, even if strength values vary. Therefore, in attempting to estimate tip resistance from shear strength values, it appears appropriate to decrease the magnitude of the cohesion term by $20 \%$.

Also, previous discussion on cone effective area suggested that for a standard cone of diameter $36.7 \mathrm{~mm}$ in snow of mean grain size $\sim 0.7 \mathrm{~mm}$, the effective area of the cone will likely be greater than the actual cone cross-sectional area (by perhaps $\sim 7 \%$ ) thus resulting in potential over-estimation of actual resistance. Thus in attempting to estimate CPT resistance values, calculated resistances should be increased by this amount to more accurately represent expected resistance.

\subsubsection{Quantification \& verification}

Description of the terms within Equation 7.4 plus consideration of additional terms is now complete and the final form of an equation describing CPT tip resistance is presented.

In summary, the terms to incorporate are:

1. C - the shear strength of the snow,

2. S - compactive element, equal to $M C$,

3. F - friction on cone face, equal to $(\mu \mathrm{S})$,

plus two modifying factors:

4. reduction of $\mathrm{C}$ by $20 \%$ owing to rate, and

5. increase of $7 \%$ owing to effective area.

This results in the following equation:

$$
q_{c}=1.07\left[0.8\left[\left((M+1) C_{\text {vert }}\right)+\left(\mu M C_{\text {vert }}\right)\right]\right]
$$

CPT measures the vertical component of stress whereas Equation 7.12 includes components of stress normal to $(\mathrm{S})$ and parallel to $(\mathrm{F})$ the cone. Resolving $\mathrm{S}$ and 
F vertically by multiplying by $0.5\left(\cos 60^{\circ}\right)$ and $\sim 0.87\left(\cos 30^{\circ}\right)$ respectively and assuming $\mu$ is 0.1 , Equation 7.12 reduces to:

$$
q_{c}=0.856(C(0.5+0.587 M))
$$

This equation incorporates all of the terms of the original conceptual model plus modifying factors. However, strengths incorporated are shear strengths. The RH side of Equation 7.13 now needs to be multiplied by two to arrive at an estimate for snow resistance in compression (via CPT). The following final equation results:

$$
q_{c}=1.7(C(0.5+0.587 M))
$$

As means of verification, if a typical value of cohesion for snow of initial density $450 \mathrm{~kg} \mathrm{~m}^{-3}$ of $\sim 100 \mathrm{kPa}$ is considered (Mellor, 1975), then a multiplier (M) of 5.3 is obtained from Table 7.6. This results in an estimated cone tip resistance of $\sim 610 \mathrm{kPa}$. CPT Test 9 was adjacent to snow pit \#1 where snow of density $\sim 450 \mathrm{~kg} \mathrm{~m}^{-3}$ was evident between 1.0 and $1.2 \mathrm{~m}$ depth. The average tip resistance value for this depth range is $\sim 570 \mathrm{kPa}$.

Applying this more generally: the average snow density in pit \#1 to a depth of $4.7 \mathrm{~m}$ is $\sim 390 \mathrm{~kg} \mathrm{~m}^{-3}$. From Equation 7.11 a value for $\mathrm{M}$ of $\sim 3.5$ is obtained and applying Equation 7.14 to the mean depth-averaged tip resistance value for all tests at that site (1.33 $\mathrm{MPa})$ results in a mean strength estimate of $30 \mathrm{kPa}$. This lies within the range of strengths $(20-90 \mathrm{kPa}$ ) suggested by Mellor (1975) for snow of density $390 \mathrm{~kg}$ $\mathrm{m}^{-3}$. This suggests that Equation 7.14 may provide realistic estimates of tip resistance from shear strength data. Shortly, in Section 7.5, estimates for shear strength obtained using Equation 7.14 will be compared to other snow shear strength indices.

\subsubsection{Other considerations}

This method of estimating CPT tip resistances from snow shear strength data is not conclusive and further work is necessary to investigate this phenomenon further. Limited empirical evidence was available to constrain the model described above and although the snow immediately proximate to the penetrometer may increase in density to values supposed above, it is probable that in a semi-infinite space, this increase will diminish with distance away from the penetrometer, eventually returning to that of the parent material at an unknown distance commensurate with the extent of the plastic pressure bulb extended normally from the penetrating cone.

Some measure of this distance may be gained by examining work in Muro and O'Brien (2004) where in a semi-infinite space, the thickness of the plastic deformation zone ahead of a penetrating plate in snow can be estimated by the following equation:

$$
T=K X
$$


where $\mathrm{T}$ is the thickness of the plastic zone, $\mathrm{X}$ is penetration depth and $\mathrm{K}$ is the coefficient of propagation of plastic compression defined by:

$$
K=2.95 \rho_{0}^{-3.933}
$$

where $\rho_{0}$ is density in $\mathrm{g} \mathrm{cm}^{-3}$. For a penetration depth of $\sim 18 \mathrm{~mm}$ (approximately half the cone diameter) and an initial density of $450 \mathrm{~kg} \mathrm{~m}^{-3}$, the thickness of the plastic zone equals $\sim 3$ to $\sim 3.5$ (via extrapolation of tabled data within Muro and O'Brien (2004) as the equation above appears not to represent tabled results), suggesting that the zone of plastic deformation during CPT might extend into the surrounding snow by a distance of $\sim 50$ to $\sim 60 \mathrm{~mm}$. This is consistent with expected pressure bulb extent via both Boussinesq and Stehle's work in snow (Stehle, 1970) but it is inconsistent with empirical evidence from CPT at Halley. The difference in thickness of the deformation zone is expected to be due to the very brittle behaviour of dry bonded polar snow whilst undergoing CPT as opposed to the plastic behaviour that may be experienced at much lower loading rates. Further investigation into the extent of this deformation zone is of interest, however, additional refinements are not investigated here.

The approximate derivation of CPT tip resistance from snow strength data at an assumed initial density is promising. However, snow density must be known to apply this estimate. Is there any way that a similar correlation can be derived without prior knowledge of density, purely using data obtained from CPT?

\subsubsection{Estimating strength solely from CPT}

The conceptual argument and equations presented thus far allow estimation of probable snow shear strength directly from CPT, although knowledge of snow density is still necessary in order to estimate a multiplier, M. As shown previously in Table 7.6, small variations in initial density can significantly alter the value of $M$, and any generated strength estimate.

If no direct measure of density is possible then density could be estimated from environmental data such as temperature and accumulation, and errors and uncertainties could be quantified; models such as that by Jun and Zwally (2002) could be considered. Alternatively, can density be derived directly from CPT friction sleeve data?

It was previously shown that sleeve friction appears to increase with snow density. Although it is suggested in subsection 7.4.2.2 that this variation may be 'damped' (because of the decrease in density variability post-compaction) it is still expected that a qualitative variation of density can be obtained by observing the variation of friction measured via CPT with depth. Figure 7.5 suggested a correlation between density and sleeve friction, and the cross correlation coefficient between the two functions is up to $\sim 0.63$. However, this is a poor method of measuring the relationship between two such averaged variables. Can a quantitative assessment of density be made from sleeve 
friction?

A linear line of best fit can be applied to an X-Y scatter plot of density versus average friction such that a relationship between the two variables can be established. However, the imprecision of the measurements, particularly density, do not allow a confident application of this method at this time. It is proposed that in conjunction with a higher resolution density trace (such as that obtainable via neutron probe (Morris and Cooper, 2003)) a stronger correlation between sleeve friction via CPT and density could be established. This would mean that independent density measurements may not be necessary, and density estimates to enable calculation of the density-dependant strength multiplying parameter could be calculated, thus allowing derivation of an estimated snow strength proxy directly from CPT. Ideally, testing would occur in homogeneous snow of known density over a temporal period, allowing exploration of the relationships between density, sleeve friction (tip resistance also) and evolving snow microstructure.

This concludes the conceptual consideration of cone tip resistance from snow shear strength. Now, Equation 7.14 is applied to CPT tip resistance data obtained at Halley to enable comparison of estimated shear strength from CPT with other strength proxies.

\subsection{Comparison with strength data}

Is estimated strength via CPT consistent with estimates for the Rammsonde, a strengthproxy assessing tool that has been used since the 1930s? Rammsonde values (normalised with average cone tip resistance to enable comparison, derived from data within Abele (1990)) and estimated CPT tip resistance values for snow of density $\sim 550 \mathrm{~kg}$ $\mathrm{m}^{-3}$ are both plotted against shear strength on a logarithmic scale in Figure 7.13.

The curves are of similar magnitude because ramm number was normalised with average tip resistance and are of similar shape and form. This suggests that estimates of CPT tip resistance from shear strength using Equation 7.14 generate a range of values consistent with those obtainable via Rammsonde.

Confined compression tests were conducted on numerous samples extracted from snow pit \#1; this was outlined in Chapter 6 . A comparison of the mean of these strengths with mean compressive strengths derived from the CPT tip resistance trace is presented in Figure 7.14. The x-axis represents depth at irregular intervals to $2.9 \mathrm{~m}$ (depths at which cores for compression testing were sampled).

Mean strength values (thin horizontal lines) differ by only $\sim 15 \%$ and are not statistically different at the $95 \%$ confidence level (via unpaired t test). This comparison suggests that representative compressive strength values may be obtained using Equation 7.14. Apparent differences may be because of:

1. Different data sampling. Compressive testing was conducted on samples ex- 


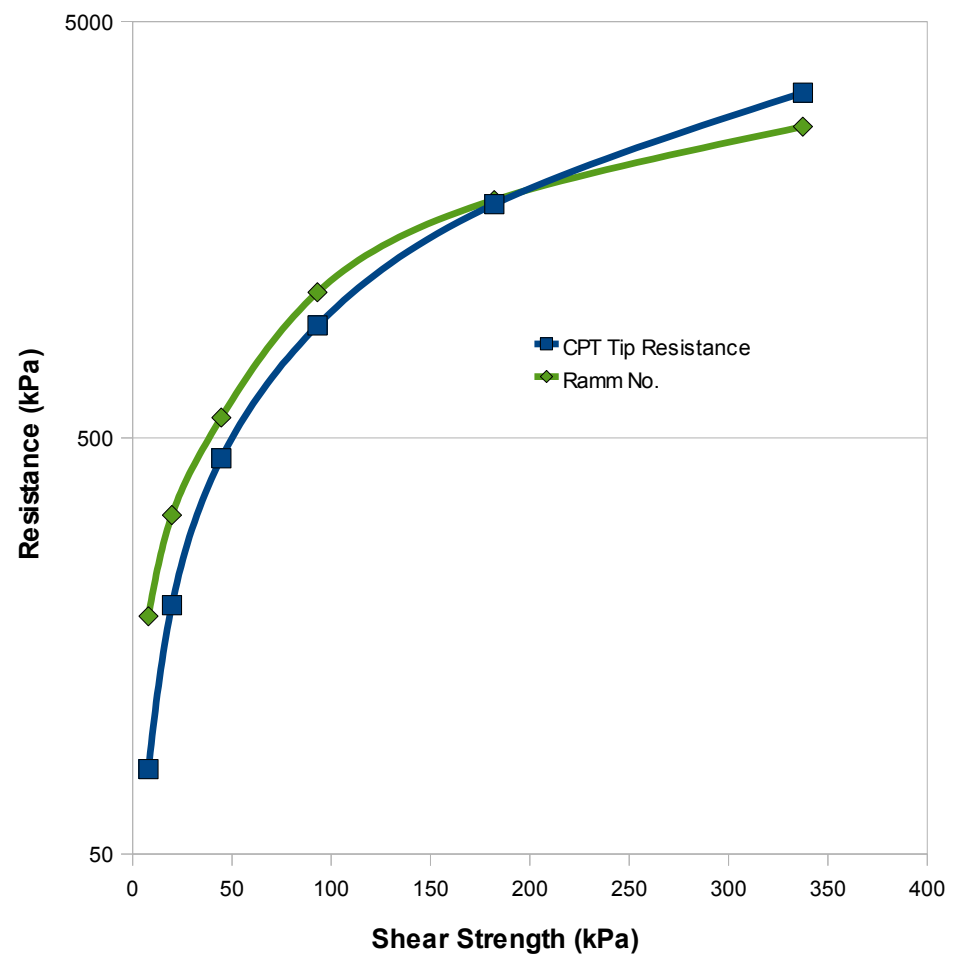

Figure 7.13: Estimated tip resistance and Ramm hardness versus snow shear strength. The curves are of similar shape and form.

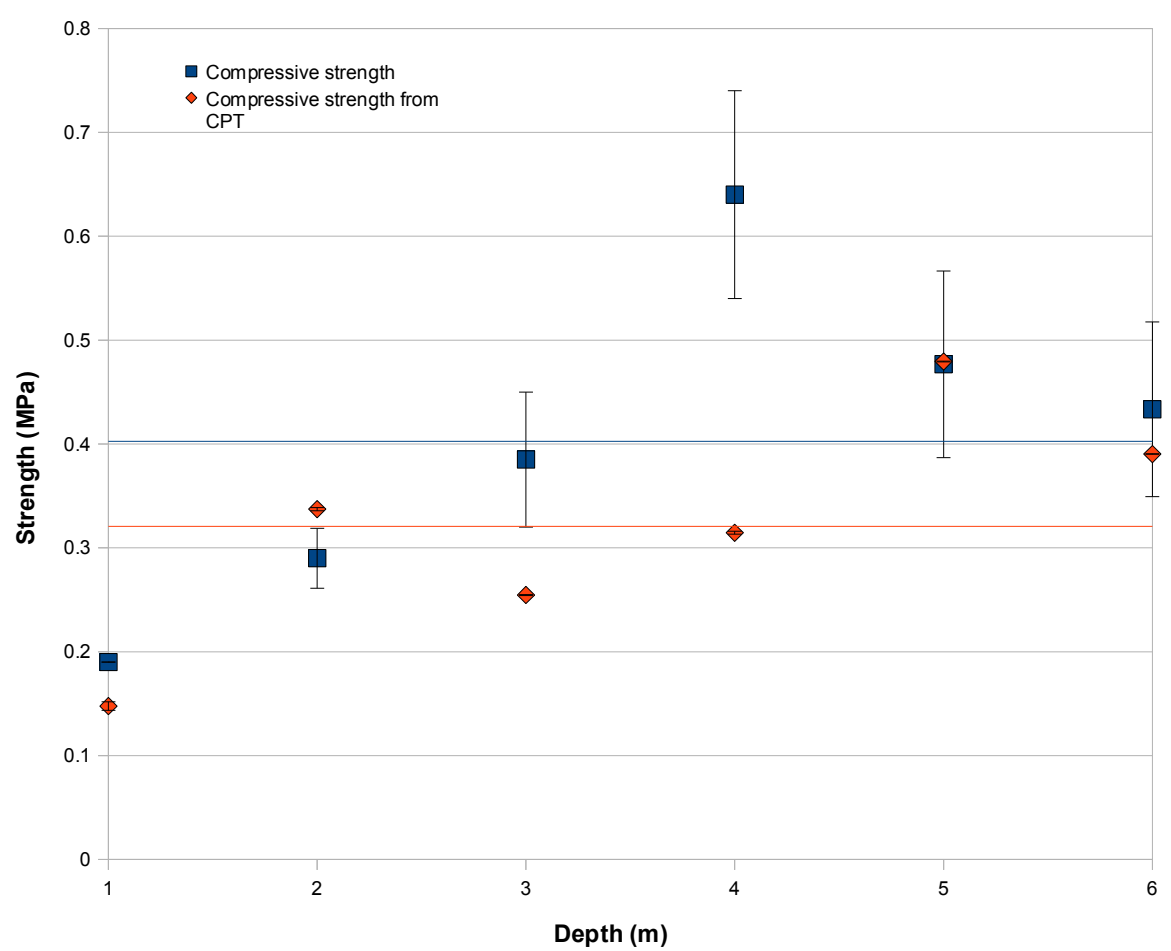

Figure 7.14: Mean compressive strength from compressive strength tests at discrete depths compared with mean depth-averaged compressive strength derived from CPT; standard error shown where possible.

tracted from discrete depths, whereas the compressive strength from CPT is derived from averaging continuous CPT data over equivalent depths; 
2. Rate. The tests were conducted at different rates, hence variation may be expected;

3. Confinement. Both tests were on semi-confined snow although the compression tests were conducted within a rigid sleeve, hence differences in confinement may have contributed to variations;

4. Flat plate vs cone. CPT strength data was derived from a cone whereas the compressive testing used a flat plate; this may cause some difference, although geometry effects are considered in deriving strength from CPT resistance, and

5. Natural variability. Snow displays large natural variability in strength hence variation between these compared values is not unexpected.

Shear strength testing at Halley was only conducted on surface layers, hence limited comparison between CPT derived strength values and measured shear strength values is possible. Owing to the difficulty in identifying comparable test sites, no direct comparison can be made and only a general assessment of average values is possible (Table 7.7).

Table 7.7: Comparison - average shear strengths and average tip resistance - Halley Vehicle Park.

\begin{tabular}{|c|c|c|}
\hline Average Shear & Average Tip Resis- & Average Shear Strength \\
\hline $\begin{array}{l}\text { Strength } \\
(\mathrm{kPa})\end{array}$ & $\begin{array}{l}\text { tance }\left(\begin{array}{lll}\text { top } & 0.1 & \mathrm{~m}\end{array}\right) \\
(\mathrm{kPa})\end{array}$ & $\begin{array}{l}\text { from tip } \\
(\mathrm{kPa})\end{array}$ \\
\hline 40 & 400 & 43.5 \\
\hline
\end{tabular}

In this table the average value for all the shear strength tests in the Halley Vehicle Park is compared with shear strength derived from the average tip resistance (to $0.1 \mathrm{~m}$ depth) for the initial 9 tests in the Halley Vehicle Park (excluding flat plate tests). The divisor to calculate shear strength from tip resistance (9.2) is derived from an average snow density of $550 \mathrm{~kg} \mathrm{~m}^{-3}$.

The average shear strength estimated from CPT agrees very well with shear strength measured via direct shear tests at Halley. Differences are expected because of differences in strain rate, size effects and the difficulties in obtaining accurate shear strength data in hard snow using the shear box. This comparison with direct shear strength data is favourable and estimated shear strength values are also consistent with published data within Mellor (1975).

Now that a conceptual model for the estimation of snow shear strength from CPT has been developed, Johnson's statistical micromechanical model (Johnson, 2003) is reinvestigated in an attempt to more precisely identify its suitability or otherwise for use with CPT. 


\subsubsection{Comparison with statistical micromechanical model}

As discussed in section 7.4.1, Johnson (2003) developed a model for penetration in granular materials that combines: available number of particles in contact with a penetrometer, the probability that these particles are in contact, and the average strength of each of these elements; his final equation for maximum cone penetration force plus nomenclature is at Appendix C. Some of the parameters for this statistical micromechanical model can be defined from my CPT observations, except perhaps the value of $f_{\perp r}$, the "microstructural element failure force normal to the penetrometer surface", essentially the strength of each element (ice grain / bond) in contact with the penetrometer.

This value is equal to $k_{\perp} . \delta_{\perp r}$, an elastic constant for the material multiplied by the element deflection at failure. This value can be estimated for CPT by assuming $\mathrm{E}$ (for ice, the microstructural element component) equals $\sim 1000 \mathrm{MPa}$ and strain at failure is 0.003. For an average grain length of $\sim 0.8 \mathrm{~mm}$ (as observed at Halley), this results in a value for $f_{\perp r}$ of $\sim 12 \mathrm{~N}$. This is the force at which a microstructural element will fail, normal to the penetrometer. If Johnson's equation and values presented at Appendix $\mathrm{C}$ are then used, this results in a maximum penetration force $\left(F_{p m}\right)$ of $\sim 6 \mathrm{~N}$, which over the area of the $35.6 \mathrm{~mm}$ diameter cone translates to a resistance of $\sim 6 \mathrm{kPa}$.

This value approaches the same order of magnitude of modelled values suggested by Johnson for larger penetrometers $(\sim 30 \mathrm{kPa})$ yet is far lower than measured CPT tip resistance values in snow, which are of the order 600 to $700 \mathrm{kPa}$ for snow of density 450 $\mathrm{kg} \mathrm{m}{ }^{-3}$. What is the reason for this substantial difference? Is it because compaction is directly incorporated within my conceptual model whereas it is incorporated via probability within Johnson's model? Although shear strength values derived via my conceptual method are equivalent in magnitude to resistances suggested by Johnson's model, CPT resistance values remain an order of magnitude higher. Johnson's model is sensitive to the microstructural dimensions chosen for the model $\left(L_{\perp} \& L_{\|}\right)$and is also particularly sensitive to the estimated contact probability. If the probability of contact is increased from 0.001 to 0.003 (consistent with Gibson and Ashby (1997) as described within Johnson (2003)), and the microstructural element dimensions are rotated by $90^{\circ}$ (a valid assumption) then resistance values approaching those obtained via CPT can be obtained by using Johnson's statistical micromechanical model.

Essentially, insufficient physical microstructural data were obtained to enable constraint of my estimated tip resistance values via Johnson's statistical micromechanical model. However, manipulation of model input $\left(L_{\perp} \& L_{\|}\right)$, still within feasible ranges suggests that values obtained via this method may be consistent with measured CPT tip resistance values. 


\subsubsection{Estimating sleeve friction}

All discussion in this chapter thus far has pertained only to forces on the cone tip; sleeve friction has not yet been considered. Equation 7.4 defined tip resistance in terms of: cohesion, a compactive term, and a small frictional component, but can this equation be used to estimate sleeve friction?

The components of equation 7.4 might be modified in the following manner:

1. C - cohesion; not relevant as all snow adjacent to the friction sleeve is assumed fractured;

2. S - compactive term, equal to $M C$; still relevant to provide estimate of normal force; to be resolved horizontally not vertically;

3. F - friction on cone face, equal to $\mu \mathrm{S}$; still relevant; this is the sleeve friction term;

4. reduction of $20 \%$ owing to rate; inconclusive evidence hence not considered; and

5. increase of $7 \%$ owing to effective area; not relevant.

Thus an equation of the following form may provide an estimate for sleeve friction from snow strength:

$$
f_{s}=\left[M C_{\text {horiz }}\right] \mu
$$

which assuming $\mu$ is 0.1 and resolving $\mathrm{S}$ horizontally by multiplying by $\sim 0.87$ reduces to:

$$
f_{s}=0.087 M C
$$

If a typical value of cohesion for snow of initial density $450 \mathrm{~kg} \mathrm{~m}^{-3}$ of $\sim 100 \mathrm{kPa}$ is used (Mellor, 1975) along with a multiplier (M) of 5.3 from Table 7.6 then an estimated sleeve friction of $46 \mathrm{kPa}(0.046 \mathrm{MPa})$ is obtained. This is two orders of magnitude greater than variations observed at Halley, but this is because the force derived from Equation 7.18 is a force mobilised during compaction and is not the normal force on the friction sleeve post compaction. Manipulation of equation 7.4 does not allow accurate estimation of CPT friction sleeve resistance. Post-compaction normal forces acting on the friction sleeve can be estimated by examining the elastic modula of the snow post-penetration.

With negligible compaction (as in the pre-drilled hole experiments) the snow density will remain the same as the virgin snow (say $450 \mathrm{~kg} \mathrm{~m}^{-3}$ with $\mathrm{E}=\sim 600 \mathrm{MPa}$ ) whereas if additional material is compacted into an annulus then density may increase to $\sim 600$ $\mathrm{kg} \mathrm{m}^{-3}$ (as presented in Table 7.6) and the modulus will increase to $\sim 2000 \mathrm{MPa}$. This approximately three-fold increase in elastic modulus means that for any normal 
loading of the friction sleeve, increased elastic stress can be up to three times greater when compaction of snow into the annulus is considered. This approximately three-fold increase in normal stress can be seen in Figure 5.25 where average sleeve friction was seen to increase from $\sim 0.4 \mathrm{kPa}$ in the pre-drilled holes to $\sim 1.2 \mathrm{kPa}$ in a standard test.

The conceptual model derived in this chapter is not useful in estimating CPT sleeve friction values but variations in observed sleeve friction post-compaction are shown to be quantitatively rational.

\subsection{Summary}

This chapter showed that existing methods of estimating snow shear strength from CPT tip resistance were inadequate. A conceptual model based on empirical observations at Halley, incorporating snow cohesion, compressibility and friction on the cone surface was then proposed. Tip resistances estimated via this method are similar to measured tip resistances obtained at Halley, and snow strengths derived via the model agree well with strength proxies obtained at Halley and published data. The conceptual model proposed herein appears to provide a good estimate for snow strength from CPT data. In the next chapter, both direct and indirect methods of deriving bearing capacity from CPT data are investigated. 


\section{Chapter 8}

\section{Application of CPT data}

One of the main aims of this research was to investigate the practical application of CPT data. An estimate for strength from CPT was established in Chapter 7 and sufficient understanding has been gained to warrant application; an obvious use being to estimate surface bearing capacity. This chapter is not an exhaustive examination of bearing capacity in polar snow but it discusses how the surface bearing capacity may be estimated through the application of CPT data.

Since the development of the CPT, analogy has been made between the cone penetrometer and driven piles, to assist in the design of deep (piled) foundations, however, here the emphasis will be on surface loads and shallow foundations; foundations that are at a depth not greater than the width of the surface structure.

Derivation of a surface bearing capacity from CPT resistance is particularly interesting because CPT resistances are derived via brittle fracture and compaction of the snow pack, whereas bearing capacity implies a non-dynamic surface loading such that, unless instantaneous fracture and settlement were to occur, deformation and settlement over extended temporal regimes will be dominated by creep processes, very different behaviour to that considered within this work thus far. Bearing capacity is now defined and then methods for deriving bearing capacity from CPT are explored.

\subsection{Definitions}

Prior to the pore close-off density of $\sim 840 \mathrm{~kg} \mathrm{~m}^{-3}$ snow essentially consists of a matrix of linked three-dimensional "chains" of ice (Gubler, 1978). Upon load application, stresses will be transmitted through this system and elastic strain will result. On a microscale some fracturing and compaction of grains is likely, resulting in load intensification and settling. This will result in densification of the snow upon the macro level. Over a long time period the majority of settlement will occur via creep processes, but in the short term some fracture and compaction under an applied surface load is likely. In summary, when a load is placed on snow, three things can happen: 
1. sufficiently small loads will merely produce minor elastic deformation and no irreversible compaction,

2. heavier loads will, over a short period of time, cause fracturing of the grain bonds, and compaction will occur until sufficient resistance is mobilised to bear the applied load; termed primary (short-term) consolidation settlement, or

3. heavier loads will, over longer periods of time, experience primary consolidation settlement, and then continue to settle further as creep processes occur; this is secondary (long-term) consolidation settlement.

The load that can be borne in the first instance above is termed the ultimate bearing capacity: the theoretical maximum pressure that can be supported without failure; here failure is defined as any settlement beyond purely elastic. In the second and third scenarios, fracture has occurred, and the bearing capacity must be defined as an allowable bearing capacity: the maximum pressure that will cause a specified limit value of settlement. So in these two scenarios, only an allowable bearing capacity can be defined and it is defined relative to an acceptable level of settlement. Failure here would be defined as settlement beyond the defined limit.

Throughout the remainder of this chapter, these terms, allowable and ultimate will be used to describe the bearing capacity of snow where settlement is or is not permissible. Kartashov (1965) reinforces that it is the initial loading of snow that will cause most settlement, and this will also occur in a short period of time. Therefore, only ultimate bearing capacity pre-fracture and allowable bearing capacity defined relative to primary consolidation settlement will be investigated; secondary consolidation settlement due to long term creep will not be examined.

\subsubsection{Types of failure}

Traditionally, bearing capacities for soils are estimated by considering three shear failure mechanisms: general shear failure, local shear failure and punching shear failure. Numerous authors have shown that because of its high compressibility, the typical shear failure mechanism for snow is punching shear (Irwin et al. (1991), Shoop and Alger (1998), Lee and Wang (2009) etc.).

When no settlement is considered only one component contributes to the ultimate bearing capacity, assuming punching shear failure:

1. shear strength of the snow beneath the footing.

When settlement is acceptable, two components contribute to the allowable bearing capacity, assuming punching shear failure:

1. shear strength of the snow beneath the footing (or shear strength of the snow beneath the compacted material under the footing), and 
2. the increase in resistance mobilised due to compaction of fractured material beneath the footing.

These components are considered in subsequent sections, but how is bearing capacity generally derived from CPT?

\subsubsection{Bearing capacity from CPT}

Bearing capacity is generally derived from CPT data in two ways (Eslami and Gholami, 2006):

1. Indirect - material parameters are derived from CPT data, and then standard bearing capacity equations are applied using these parameters, or

2. Direct - bearing capacity is derived directly from CPT data after consideration is given to shape, size, rate differences etc. by applying modification factors.

Indirect methods that are used include the application of standard bearing capacity theory, cavity expansion theory and the use of numerical models. Various approaches for direct bearing capacity derivation in soils are described in Lunne et al. (1997), including those by Schmertmann (1978), Meyerhof (1976) and Tand et al. (1995). Additional methods include Owkati (reference data unobtainable) quoted by Eslami and Gholami (2006) and a direct method derived from Rammsonde data in snow (Irwin et al., 1991).

With a knowledge of expected snow behaviour as outlined in Chapter 2 and an estimate for snow strength derived in the previous chapter, indirect methods of establishing bearing capacity from CPT are now addressed. Direct methods are then briefly examined.

\subsection{Indirect assessment of bearing capacity}

\subsubsection{Homogeneous snow - ultimate bearing capacity}

The standard elastic solution for a circular surface load on a semi-infinite mass shows that at a point beneath the centre of the load, vertical stress $\left(\sigma_{z}\right)$ equals the applied distributed load at the load-surface interface (Poulos and Davis, 1974). So, as a first estimate in homogeneous snow, the ultimate bearing capacity will be reached when this vertical stress surpasses the compressive strength of the snow. The compressive strength of snow was earlier considered to be equivalent to twice the shear strength of the snow (assuming negligible confinement), hence an estimate for the ultimate bearing capacity for snow under vertical loading is as below: 
where $C$ is the snow cohesion. Terzaghi's general shear failure criterion (Terzaghi, 1943) when adapted to estimate punching shear resistance reduces to a similar form for surface strip footings (once overburden and the existence of radial slip surfaces are ignored). Both of these estimates assume that the failure surface beneath the load is a wedge or cone that extends at an angle of $45^{\circ}$ internally beneath each corner of the footing. Now, how can this capacity be estimated from CPT data?

Discussion on volumetric straining in the previous chapter suggested that cone tip resistance during deformation (for snow of initial density $\sim 450 \mathrm{~kg} \mathrm{~m}^{-3}$ ) may be approximately six times greater than snow shear strength owing to stress increases during compaction (see Equation 7.14). Therefore, an estimate for the compressive strength and thus ultimate bearing capacity can be obtained from CPT data by dividing the steady-state tip resistance value in snow by $\sim$ three. If mean tip resistance was $\sim$ $2 \mathrm{MPa}$, then bearing capacity may be $\sim 670 \mathrm{kPa}$, or $\sim 33 \%$ of mean measured tip resistance. This is similar in magnitude to derived values for bearing capacity in sand which range from $\sim 10$ to $\sim 30 \%$ of CPT tip resistance values and appears reasonable. Note that the above derivation for snow assumed cohesion between particles and no friction whereas the estimates for sand assume a friction angle but no cohesion.

In summary, this assessment of ultimate bearing capacity in homogenous snow results in an equation relating ultimate surface bearing capacity (BC) and average tip resistance $\left(\mathrm{q}_{\text {cavg }}\right)$ derived from CPT of the form:

$$
B C=2\left(q_{\text {cavg }} / 1.7\right) /(0.5+0.587 M)
$$

This equation is the same as Equation 7.14 except the RHS has been multiplied by two to derive a strength in compression from CPT data. $M$ is the multiplier defined in Table 7.6 (or via Equation 7.11) for snow of various densities.

Irwin et al. (1991) in predicting the load carrying capacity of snow from Rammsonde hardness data suggested that the pressure bulb beneath a circular footing on snow can be identified as a cylindrical surface where shear resistance is provided around the circumferential perimeter of the cylinder in addition to compressive resistance provided to the bottom of this cylinder. Figure 8.1 illustrates (a.) the shear surfaces inclined beneath a surface load as is assumed for typical punching shear failure, and (b.) the additional failure surfaces suggested by Irwin et al. (1991).

Inspection of Figure 8.1 b. suggests that if a cylindrical pressure bulb is assumed then there will be additional resistance to surface loading by an amount equal to the shear strength multiplied by the circumferential area of the surface load, the dimensions of which will vary with footing size and the vertical extent of the pressure bulb. An estimate for the ultimate bearing capacity for snow under a circular footing experiencing vertical loading may then be:

$$
\text { Ultimate bearing capacity } \approx 2 C+(2 \pi r d) C
$$


a.

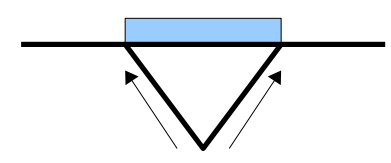

b.

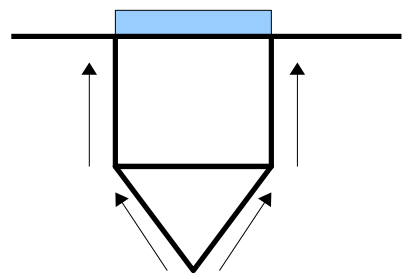

Figure 8.1: (a.) Typically punching shear failure is estimated by assuming a conical failure surface beneath the footing. (b.) Compare this with Irwin et al. (1991) who propose a circumferential shear area plus additional compressive resistance at the base of the cylindrical pressure bulb. Such resistance may be mobilised in assessing allowable bearing capacity but not in assessing ultimate bearing capacity.

where $C$ is cohesion, $r$ is the footing radius (m) and $d$ is the depth (m) of the pressure bulb. However, this formulation assumes that the vertical stress applied by the footing is distributed in its entirety throughout the snow pack, both to the base of the pressure bulb and around the circumference of the supposed cylindrical pressure bulb, but this is not the case.

The vertical stress imposed by a surface load at the load-surface interface may be assumed to be resisted by inclined shear planes as in Figure 8.1 a., or it may be assumed to be resisted by the circumferential perimeter of the cylinder beneath the surface load, but in an ultimate bearing capacity analysis both components cannot contribute. Such contribution may occur in an allowable bearing capacity analysis as described in the next sub-section.

To assess the extent of resistance offered by the circumference of the supposed cylindrical pressure bulb, both the depth of the pressure bulb and radius of the load must be known. Rather than attempt to derive these parameters, some estimate for the ultimate bearing capacity of a circular footing assuming a cylindrical pressure bulb may be made by comparing the surface area of a conical zone beneath a circular plate with the surface area of a proportionately dimensioned cylinder. Geometry shows that resistance offered by the circumference of a cylindrical pressure bulb will be $\sim 1.4$ times greater than that provided by a conical wedge of $45^{\circ}$, which implies that the ultimate bearing capacity of a vertical surface load may also be estimated from CPT by Equation 8.4 which is equivalent to the RHS of Equation 8.2 multiplied by 1.4:

$$
B C=2.8\left(q_{\text {cavg }} / 1.7\right) /(0.5+0.587 M)
$$

Therefore, the ultimate bearing capacity of homogeneous snow is estimated to be $\sim$ 2 to 3 times greater then the shear strength of the snow, and estimates can be obtained indirectly from CPT data. The allowable bearing capacity of homogeneous snow where primary consolidation occurs is considered next. 


\subsubsection{Homogeneous snow - allowable bearing capacity}

In the previous subsection it was shown that the ultimate bearing capacity is approximately equal to the mean steady-state cone tip resistance divided by $\sim$ three (for snow of initial density $\sim 450 \mathrm{~kg} \mathrm{~m}^{-3}$ ). In this section the bearing capacity after settlement or primary compaction is investigated.

Once the applied compressive stress surpasses the strength of the snow at the surface, primary compaction will occur. Settlement and fracture will continue until sufficient resistance is mobilised to oppose the applied load. This compaction will occur in a staggered manner as individual bonds fail, resulting in the typical 'saw-tooth' shaped pattern of load vs displacement exhibited by snow compacting under load. The fractured material acts as a load transferrer, transferring the load to snow at depth, so that on the macro-level, the total resistance will be due to the compressive strength of the snow at depth and the increase in resistance due to primary compaction.

Two methods are now considered to estimate the increase in strength gained during primary compaction:

1. empirical volumetric compaction data is considered as a means of estimating the increased allowable bearing capacity once settlement has occurred, and

2. the Mohr-Coulomb model is applied in conjunction with volumetric reasoning to estimate strength of a fractured granular material.

In order to estimate an allowable bearing capacity, failure must be defined, as a limit of settlement. During his flat plate tests Abele (1970) defined 'failure' after compaction of $\sim 0.3$ inches $(\sim 8 \mathrm{~mm})$, for a flat plate of radius 3 inches, in snow of initial density $450 \mathrm{~kg} \mathrm{~m}^{-3}$. The allowable bearing capacity that Abele (1970) recorded during settlement to this limit was $\sim 420 \mathrm{kPa}$. The unconfined compressive strength of Abele's processed snow was $\sim 270 \mathrm{kPa}$, so the allowable bearing capacity recorded by Abele (1970) can be expressed as a $155 \%$ increase over the ultimate bearing capacity.

Irwin et al. (1991) presents data that shows for snow of density $340 \mathrm{~kg} \mathrm{~m}^{-3}$ and $480 \mathrm{~kg} \mathrm{~m}^{-3}$, aged for between 2 hours and 16 days, the allowable bearing capacity due to compaction was $\sim 300 \%$ greater than the ultimate bearing capacity. Such variation between Abele (1970) and Irwin et al. (1991) is not unexpected as the snow used differed in density and age; Abele (1990) shows that snow of initial density $450 \mathrm{~kg}$ $\mathrm{m}^{-3}$ at $\sim-10^{\circ} \mathrm{C}$ can develop twice as much strength at two weeks age than it possessed at one.

So, indirectly, strength data from CPT (Equation 7.14) can be used with existing empirical evidence to derive an approximate allowable bearing capacity for snow once an ultimate bearing capacity has been estimated. Consideration of this process in the context of the Mohr-Coulomb model occurs next. 
Prior to fracture the shear strength of the snow consists solely of the cohesive component: no movement has occurred therefore no friction is mobilised. Upon fracture of the ice matrix, loss of cohesion occurs on a micro level, compaction of the now particulate snow occurs, and friction between grains is mobilised proportional to the applied normal stress; some frictional contribution may be mobilised as partial cohesion is lost, consistent with work by Hajiabdolmajid et al. (2002) on the brittle failure of rock. In snow, it is likely that substantial compaction towards the critical density of $\sim 550 \mathrm{~kg}$ $\mathrm{m}^{-3}$ must occur before sufficient grain to grain contact occurs to enable substantial frictional resistance. As was assumed when considering volumetric compaction, the total resistance will be due to the elastic contribution of the snow at depth and the increase in resistance due to frictional processes. These frictional processes are now considered further.

The shear strength $\tau$ of the snow undergoing compaction can be expressed as $N \tan \phi$ where $N$ is the normal stress and $\phi$ is the kinetic friction angle for the snow. The snow is cold and unbonded and may possess a static friction angle of perhaps 35 $40^{\circ}$ (McClung and Schaerer, 1993) and thus a kinetic friction angle of perhaps $25-30^{\circ}$ (shown by laboratory studies to be $\sim 10^{\circ}$ less than the static friction angle (McClung and Schaerer, 1993)); the tangent of such a kinetic friction angle equals $\sim 0.5$. So, because $\tau=N \tan \phi$, to realise a shear strength equivalent to that mobilised at initial failure requires a normal stress equal to twice this amount. Excluding dynamic effects, after the initial ultimate bearing capacity has been surpassed, coincidentally, the same amount of bearing capacity can be mobilised purely by frictional processes: $N \approx 2 \tau$.

The total increase in resistance will be due to this frictional component, plus an elastic component owing to the cohesive snow beneath the compacted layer. This elastic component is considered equivalent to the ultimate bearing capacity and equals $2 \mathrm{C}$, so the resultant allowable bearing capacity will therefore be of the order of $\sim 200$ $\%$ greater than the original compressive strength or ultimate bearing capacity of the snow.

To summarise: by considering empirical bearing plate evidence, a $\sim 150$ to $300 \%$ increase in bearing capacity over the initial ultimate bearing capacity of the snow is expected and by considering a Mohr-Coulomb model an increase of $200 \%$ is expected. It would appear that allowable bearing capacity can reasonably be estimated to be approximately twice the ultimate bearing capacity. This estimate is for snow of initial density $450 \mathrm{~kg} \mathrm{~m}^{-3}$ and will vary with the size and shape of the loaded area and the density, age and thus microstructure of the snow. This increase in bearing capacity comes at the cost of settlement, which may or may not be acceptable depending on the application. Further consideration of primary consolidation settlement is not considered here. It has been briefly explored to highlight that in any bearing capacity scenario upon snow, unless the snow has been processed and compressed to increase compaction and density in surface layers then some level of settlement must be ex- 
pected upon the application of surface loads. All of the discussion above pertains to an assumed homogeneous snow mass; in natural layered snow additional considerations must be addressed.

\subsubsection{Layered snow}

For the calculation of bearing capacity in thinly layered strata such as snow, Dean (2010) suggests that an averaging procedure be used. The material should be treated as a two-layer medium, with multiple lower layers replaced by a single layer with equivalent bearing capacity.

During penetration towards a harder layer a 'squeezing' motion can develop (Dean, 2010) where the stress is distributed within the upper softer layer. This behaviour was demonstrated by Marshall (2005) in studies on the load of a skier on a layered snowpack. Dean (2010) notes that "generally, a hard stratum overlying a weaker one presents a danger that a foundation intended to rest on the hard stratum may fail by punching through into the weaker one" and Meyerhof (1948) outlines a decrease in the possible end bearing capacity of a pile depending on its proximity to weaker lower layers. In layered snow where ice and hoar layers may coexist both 'squeezing' and 'punching' can occur, so caution needs to be used in defining and applying bearing capacity formulations to such systems.

For homogeneous snow where snow at depth has the same strength as surface snow, non-failure at the surface implies non-failure throughout, yet if a weaker layer existed at depth and the percentage of stress transfer exceeded that strength then failure might occur at that layer. To assess the extent of vertical stress transfer within the snow and address these 'squeezing' and 'punching' mechanisms, the elastic modulus for each layer of snow must be known. So, before bearing capacity calculations in layered snow can be considered, the derivation of Young's modulus from CPT data must be examined.

\subsubsection{Estimating Young's modulus from CPT}

Young's Modulus $(E)$ is the ratio of uniaxial stress over uniaxial strain, typically derived from the slope of the origin to failure-point line in a stress/strain curve. Russell-Head and Budd (1989) estimate the elastic modulus for each layer of a snow pavement from California Bearing Ratio (CBR) values. In conjunction with an additional empirically derived relationship between density and CBR, this allows a power-law relationship between density and an effective elastic modulus to be established:

$$
\bar{E}_{\text {eff }}=3.28 \times 10^{4} \rho^{9.02}
$$

where $\bar{E}_{\text {eff }}$ is effective elastic modulus and $\rho$ is density $\left(\mathrm{Mg} \mathrm{m}^{-3}\right)$. At a density of $0.45 \mathrm{Mg} \mathrm{m}^{-3}$ an effective elastic modulus of $\sim 24 \mathrm{MPa}$ is derived. However, this 
method precludes the use of CPT data hence alternative methods of estimating the modulus are preferable.

Johnson and Schneebeli (1999) as part of their statistical-micromechanical model for the SMPT obtain a value for the microscopic elastic modulus by deriving a stiffness from the average failure strain and force of microstructural elements; they then relate this micro-modulus to a macro-modulus by considering the number of elements engaged at a macro level, thus a macroscopic elastic modulus can be derived which is a percentage of the microscopic. Marshall (2005) refined this approach using a mean structural element volume (rather than an area) to define the macroscopic elastic modulus below:

$$
\bar{E}_{\text {macro }}=k_{\text {eff } f} \frac{A_{\text {tip }} z}{n_{r} \bar{A}_{n}^{2}}
$$

where $\bar{E}_{\text {macro }}$ is macroscopic elastic modulus, $k_{\text {eff }}$ is effective stiffness, $A_{t i p}$ is area of penetrometer tip, $z$ is the penetration distance, $n_{r}$ is the number of snow grains ruptured and $\bar{A}_{n}^{2}$ is the mean cross-sectional area of the microstructural element. Marshall (2005) concluded that "micro-scale variability is quickly averaged out as sample size increases" thus this method of estimating a value for $E^{\text {macro }}$ may prove valid for the larger penetrometer used within CPT.

Marshall (2005) applied this formula in the following manner:

1. calculate $k_{\text {eff }}$ for a cubic sample $10 \mathrm{~cm}$ on a side,

2. calculate $E_{0 \text { ( } \text { snow) }}$ from Scapozza and Bartelt (2003a) who modeled the tangent elastic modulus at strain tending to zero, $E_{0(\text { ssnow })}=0.1873 e^{0.0149\left(\rho_{\text {snow }}\right)}$, then

3. solve for $\bar{A}_{n}^{2}$ at $\bar{E}_{\text {macro }}=E_{0 \rho s n o w}$, from which representative structural element lengths (indicative of grain size) can be calculated.

By using actual tip area, average measured grain size $(0.7 \mathrm{~mm})$ and a value of $k_{e f f} / n=0.12$ (as befitting a larger penetrometer), this method can be applied to CPT resulting in an estimated $\bar{E}_{\text {macro }}$ of $\sim 40 \mathrm{MPa}$, for a sample size consistent with displaced material volume.

This value for an effective macro elastic modulus, as well as that by Russell-Head and Budd (1989) (25 MPa) is an order of magnitude less than historical data presented by Mellor (1975) that shows that for density $450 \mathrm{~kg} \mathrm{~m}^{-3}$, Young's modulus is expected to be of the order $\sim 2$ - $300 \mathrm{MPa}$. Russell-Head \& Budd's is an effective modulus derived from CBR measurements for use in their specific application, and the macroscopic modulus obtained via Marshall (2005) is also applying a microscopic statistical method to a macroscopic scenario, using estimates that may be inconsistent with the original formulation, so perhaps these methods are not appropriate for CPT. Is there any way in which an estimate of an elastic modulus can be made directly from CPT data without having to revert to a knowledge of density? 
Young's modulus was earlier defined as the ratio of uniaxial stress over uniaxial strain. A conceptual discussion in the previous chapter suggested that a value for the strength of snow can be obtained directly from CPT tip resistance data (Equation 7.14), and as a failure strain for ice is known $(\sim 0.003$, brittle compressive failure (Schulson and Duval, 2009)), is sufficient stress/strain data available to define a macroscopic value for Young's modulus upon which bearing capacity calculations can be based?

Schulson and Duval (2009) note for polycrystalline ice grains (such as found within snow) that "although the constituent crystals are anisotropic, homogeneous aggregates of randomly orientated grains are elastically isotropic" and as a result "their elastic properties can be described by only two independent constants such as W, G, B or $\nu$ ". Therefore, although there are an unknown number of particles in contact with and failing ahead of a moving penetrometer at any one time, each of these particles is composed of ice and thus will fail at a compressive strain of $\sim 0.003$. The sum of such failures on the penetrometer (minus the estimated increase in stress owing to compaction) will provide an estimate of compressive strength. Therefore, on a macro level the definition of Young's modulus as the ratio of (assumed) uniaxial stress over (assumed) uniaxial strain is sustainable, resulting in a fixed relationship between cone tip resistance and effective elastic modulus of:

$$
E_{\text {effmacro }}=\left[2\left(q_{\text {cavg }} / 1.7\right) /(0.5+0.587 M)\right] 0.003^{-1}
$$

where $\mathrm{M}$ is the multiplier defined in Table 7.6 (or via Equation 7.11) for snow of various densities and the resultant estimated shear strength is multiplied by two to obtain an estimate of uniaxial compressive strength. This relationship can be used to generate an estimate of $E_{\text {effmacro }}$ with depth (Figure 8.2).

This simple derivation of modulus is consistent with the methodology employed by Lee and Wang (2009) who in modeling snow deformation calculated yield strain in the elastic regime as merely the yield strength in compression divided by the Young's modulus. Values of effective elastic modulus extracted from Figure 8.2, at depths where density is known, agree with historical values in Mellor (1975); a modulus derived from average density values in Mellor $(\sim 130 \mathrm{MPa})$ compares well with the average modulus via $\mathrm{CPT}(\sim 150 \mathrm{MPa})$, for snow of density $450 \mathrm{~kg} \mathrm{~m}^{-3}$. Factors which may influence this estimate for effective modulus from CPT data include rate and confinement.

Scapozza and Bartelt (2003a) note that their relationship for $E_{o(\rho s n o w)}$ is strain-rate independent, which initially appears consistent with data from Mellor (1975); Mellor's data appears complementary although obtained at different rates via various techniques. This is an interesting observation and raises the question as to why a stress dependence on strain rate is observed, whilst apparently the elastic modulus is not strain rate dependant? At different strain rates, although failure strain is assumed constant $(\sim 0.003)$, stress at failure will change, thus the elastic modulus will also change. Further inspection of Scapozza and Bartelt's work (see Figure 8.3 from Scapozza and 


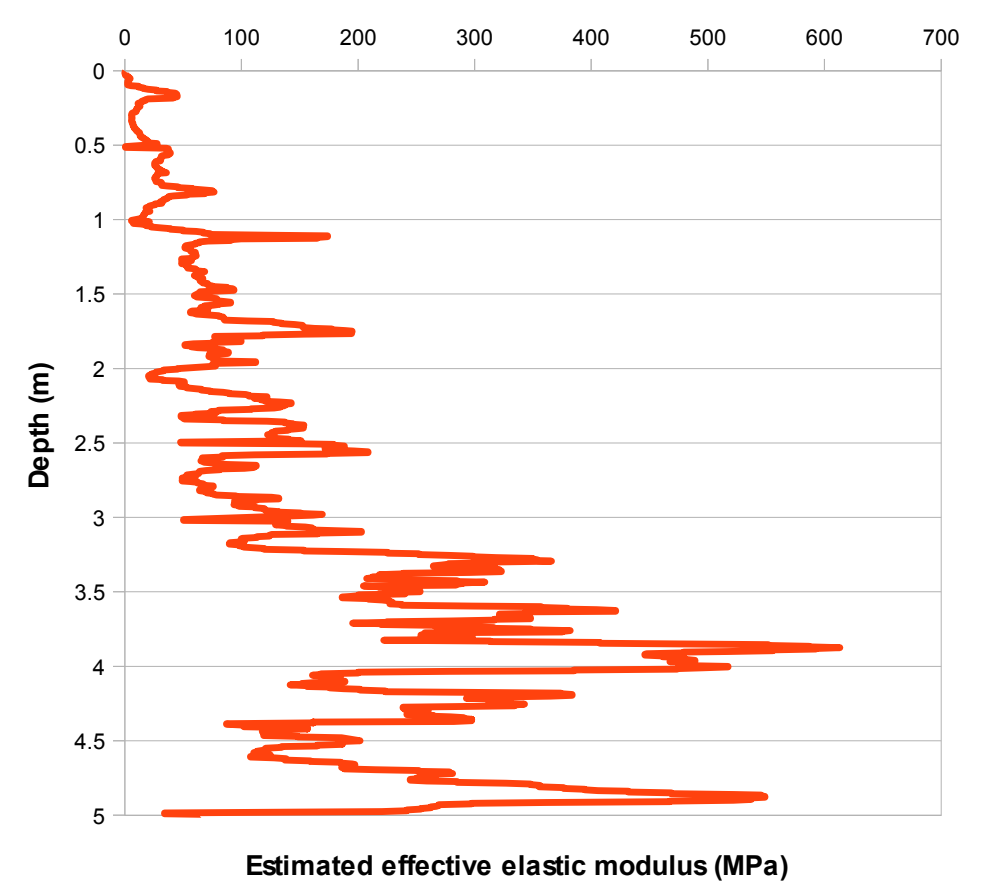

Figure 8.2: Possible variation of effective elastic modulus with depth obtained by applying Equation 8.7 to tip resistance data from Test 9, for snow of assumed density $\sim 450 \mathrm{~kg} \mathrm{~m}^{-3}$.

Bartelt (2003a)) suggests that $\mathrm{E}_{o}$ is strain rate dependent and that although Mellor's data is largely consistent, order of magnitude differences are apparent for snow of the same density at different testing rates.

So, strain rate is expected to have an effect on estimated effective modulus.

If elastic stress is transmitted through the snow instantaneously upon surface loading then $E_{\text {effmacro }}$ deduced from CPT (conducted at slower rates approaching the ductile / brittle transition) probably needs to be lowered by perhaps $20 \%$ to provide a more realistic estimate. A decrease in $E_{\text {effmacro }}$ estimated from CPT of $\sim 20 \%$ may be appropriate, such that a revised relationship between cone tip resistance and effective elastic modulus may be:

$$
E_{\text {effmacro }}=\left[1.6\left(q_{\text {cavg }} / 1.7\right) /(0.5+0.587 M)\right] 0.003^{-1}
$$

This may result in a more probable $E_{\text {effmacro }}$ estimate from CPT. An additional consideration which needs to be discussed is that of confinement.

The measurement of $\mathrm{E}$ assumes unconstrained behaviour such that expansion in the transverse direction can occur during compression, a measure defined by Poisson's ratio, which for mid-density snow equals $\sim 0.25$. If this expansion is restricted then the resulting modulus can be defined as a Constrained Modulus, M, which will be greater than E owing to the inability of the material to deform naturally. In higher density snow, some level of confinement during compaction and fracture is likely, and thus any modulus derived from this process is likely a semi-confined modulus. This 


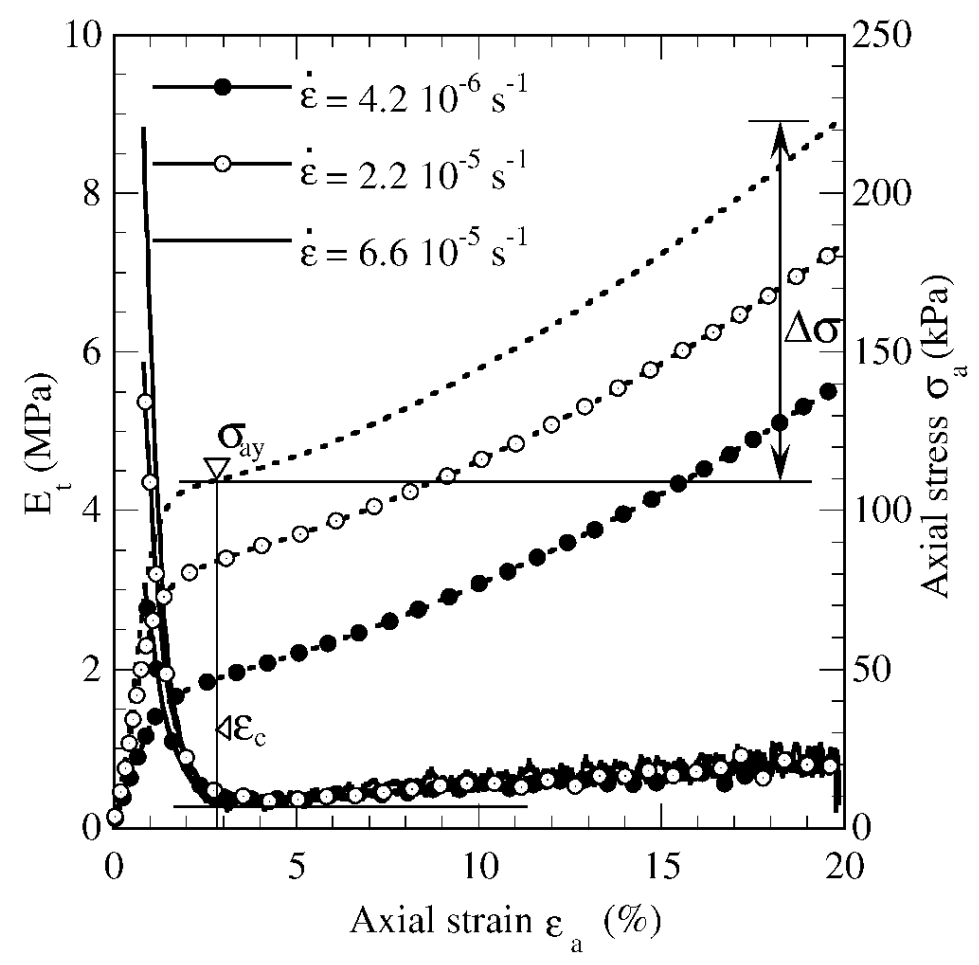

Figure 8.3: Stress-strain curves (dashed lines) and tangent modulus (continuous lines) obtained in compression tests at various strain rates (from Scapozza and Bartelt $(2003 \mathrm{a})) . \mathrm{E}_{o}\left(\mathrm{E}_{t}\right.$ at $\left.\varepsilon_{a} \approx 0\right)$ values are strain-rate dependent.

phenomenon is not further investigated but this brief discussion highlights that any effective elastic modulus derived via CPT is possibly the result of compaction under constraint, meaning the actual $E_{\text {effmacro }}$ may be less then that estimated from CPT data.

It is difficult to verify the estimate for effective elastic modulus via CPT described above with other methods. Russell-Head and Budd (1989) derived Young's modulus empirically from load/deflection data in CBR tests, and also related CBR and Rammsonde data, but they did not derive Young's modulus directly from Rammsonde data. No further method of deriving an elastic modulus from penetration testing in snow has been identified, apart from that proposed by Johnson and Schneebeli (1999), modified by Marshall (2005) as already discussed. Robertson (1991) suggested a method to derive an equivalent Young's modulus directly from CPT data but his method relies on empirical data, and is not transferable to snow. The only additional advice that can be gleaned from previous studies is based on the remark by Lee and Huang (2010) that "the indentation strength for large-diameter indenters" such as in CPT "approaches the compressive strength of the material." This is the same conclusion that was reached in Chapter 2 and is supported by other researchers (Abele (1963), Russell-Head and Budd (1989) and Wuori (1962)) hence direct consideration of tip resistance measured via CPT as a proxy for unconfined compressive strength seems appropriate for the derivation of an equivalent Young's modulus based on an assumed 
constant macroscopic failure strain.

The method of estimating $E_{\text {effmacro }}$ as described above is approximate. However, it may provide initial modulus guidance directly from a CPT test. Briaud (2001) says that a modulus defined from the testing scenario and application for which it is extracted can be defined and used within similar contexts, perhaps limiting its broader application, but enhancing application within a particular field. With an estimate for an effective elastic modulus in hand, the bearing capacity of multiple-layered environments is now considered.

\subsubsection{Layered snow - ultimate bearing capacity}

Poulos and Davis (1974) provide nomograms advising the variation in vertical stress at depth for various ratios of Young's modulus in a two-layer elastic system before presenting methods for both three and multiple layered systems; Russell-Head and Budd (1989) used these formulations in their consideration of a layered snow runway pavement. Although an averaging procedure can be used in thinly layered strata, it is these potentially weak layers, perhaps 10's of mm thick that should be captured from the CPT trace and have realistic strength assessments rigorously applied, so ideally, all identifiable layers will be considered in the bearing capacity assessment. Budhu (2000) commenting on soils, suggests that in conditions where numerous weak layers may exist, shallow foundations should be avoided, and that deep foundations (piles) should be employed. Where this is not possible he suggests using the strength characteristics of the weakest layer, and this is consistent with the approach herein. The pressure bulb testing conducted at Halley was designed to provide additional guidance on the application of this method. However, inadequate resolution was obtained and results are not incorporated into this analysis.

Before attempting to estimate surface bearing capacity in layered snow from an entire CPT tip resistance trace, various multi-layer techniques for estimating ultimate bearing capacity will first be applied to the idealised four-layer system shown in Table 8.1. $q_{c}$ values are representative of those measured during Test 9, shear strength is estimated using Equation 7.14 and $\mathrm{E}_{\text {effmacro }}$ is calculated via Equation 8.8 assuming a mean snow density of $450 \mathrm{~kg} \mathrm{~m}^{-3}$.

Table 8.1: Hypothetical layer parameters for consideration of ultimate bearing capacity in layered snow; assumed average snow density of $450 \mathrm{~kg} \mathrm{~m}^{-3}$.

$\begin{array}{ccccc}\text { Layer } & \text { Depth }(\mathbf{m}) & \text { Mean } q_{c}(\mathbf{M P a}) & \text { Shear Strength (MPa) } & \mathbf{E}_{\text {effmacro }}(\mathbf{M P a}) \\ 1 & 0-0.5 & 0.3 & 0.05 & 26 \\ 2 & 0.5-1.5 & 0.8 & 0.13 & 70 \\ 3 & 1.5-1.6 & 2.0 & 0.33 & 175 \\ 4 & 1.6-2.0 & 1.5 & 0.25 & 131\end{array}$

The premise of the strain energy method (Christiano et al., 1974) is that the elastic properties of layers within an elastic half-space are proportionate to the strain energy 
occurring in each layer. The strain energy in each layer is obtained through discrete integration of the cumulative strain energy with depth, and then the strain energy values per layer are used to weight the elastic properties of the system, resulting in an effective system stiffness. This method can be applied to as many layers as desired.

Application to the hypothetical layered system in Table 8.1, assuming a constant Poisson's ratio of 0.25 , loading under a flat rigid plate of radius $50 \mathrm{~cm}$, effective stiffness applicable to the system of $2 \mathrm{~m}$ depth, and a failure strain of $\sim 0.003$ results in an ultimate bearing capacity of $\sim 440 \mathrm{kPa}$, rising to $\sim 1.1 \mathrm{MPa}$ for a plate radius of 15 $\mathrm{cm}$.

Values calculated for similar plate dimensions using standard Boussinesq equations (Budhu, 2000), a depth-averaged value of Young's modulus and the same failure definition are $\sim 595 \mathrm{kPa}$ and $\sim 262 \mathrm{kPa}$ respectively, whilst with the same assumptions Janbu's (Janbu et al., 1956) approximation for the immediate settlement of cohesive soils produces values of $\sim 940 \mathrm{kPa}$ and $\sim 400 \mathrm{kPa}$ respectively; both methods produce results of similar order to the strain energy method.

An additional approximate solution to identify the ultimate bearing capacity of a multi-layered system is that by Palmer and Barber (1940) reproduced in Poulos and Davis (1974). Using this method, in a two-layer system the top layer is replaced with a layer of different thickness with the modulus of the lower layer material. This process can be repeated for each additional layer above the reference lower layer. Displacements within each new layer are then summed and adjusted for displacement at the equivalent depth within a homogeneous semi-infinite mass. Application of this technique results in an ultimate bearing capacity of $\sim 400 \mathrm{kPa}$ for the $0.5 \mathrm{~m}$ radius plate, and $\sim 416$ $\mathrm{kPa}$ for the $0.15 \mathrm{~m}$ radius plate.

Table 8.2 summarises the results of the various calculations described above:

Table 8.2: Summary of multi-layer elastic bearing capacity calculations.

$\begin{array}{lll}\text { Method } & \begin{array}{l}\text { Ultimate BC } \\ \text { plate) }(\mathbf{0 . 5} \mathbf{~ m a})\end{array} & \begin{array}{l}\text { Ultimate BC } \\ \text { plate) (kPa) }\end{array} \\ \text { Christiano et al. (1974) } & 440 & 1084 \\ \text { Budhu (2000) } & 595 & 262 \\ \text { Janbu (1956) } & 940 & 402 \\ \text { Palmer \& Barber (1940) } & 400 & 416\end{array}$

In an engineering application it would be typical to utilise a number of possible approaches and then likely defer to the most cautious estimate, unless it was greatly different and reasons for this discrepancy could be ascertained. For both plate sizes, the lowest derived ultimate bearing capacities are $\sim 30-45 \%$ of depth averaged tip resistance $(\sim 875 \mathrm{kPa})$ for the $2 \mathrm{~m}$ deep half-space considered. This is larger than previous percentage estimates derived for non-layered mediums (15 - 30\% of mean tip resistance) but of course these estimates depend on the values of Young's modulus employed. 
If Palmer and Barber's method (Palmer and Barber, 1940) is now applied to a (semi-) continuous CPT tip resistance trace (Test 9) down to a supposed depth of influence of $2 \mathrm{~m}$ then ultimate bearing capacities of $\sim 1.16 \mathrm{MPa}$ and $\sim 708 \mathrm{kPa}$ are obtained respectively for plates of radius $0.5 \mathrm{~m}$ and $0.15 \mathrm{~m}$. These values are greater than those within Table 8.2 probably because data in this table are derived from a simplified four-layer representation of Test 9 data, whereas a number of harder layers (resulting in increased bearing capacity) are evident down to $2 \mathrm{~m}$ in Figure 8.2.

The discussion above was on the assessment of ultimate bearing capacity in a layered medium. At surface loads greater than the ultimate bearing capacity fracture and compaction will occur. The next subsection briefly discusses the assessment of allowable bearing capacity in layered snow.

\subsubsection{Layered snow - allowable bearing capacity}

The increase in bearing capacity due to compaction in homogeneous snow was discussed earlier in subsection 8.2.2. It is envisaged that the limit settlement defined as failure will be of limited extent (Abele (1970) defined failure at settlement of $\sim 8 \mathrm{~mm}$ ), therefore the addition of more layers to the scenario has limited effect; it is assumed that the surface layer will experience the most deformation. The consideration of additional layers has been shown to affect the calculation of ultimate bearing capacity. However, when compaction is taken into account, compaction beyond the initial surface layer is not considered. In summary it is assumed that:

1. the density of the upper layer will affect the amount of compaction and settlement necessary to realise the final allowable bearing capacity due to compaction, and

2. variation in elastic modulus (and thus the transfer of stress to depth) will affect the calculation of ultimate bearing capacity, and the amount of elastic resistance provided by lower layers when considering allowable bearing capacity.

So, in assessing the bearing capacity of layered environments an increase in strength owing to compaction of the surface layer can be incorporated, and stress transferral to lower, potentially weaker layers also needs to be investigated. The methods discussed in the two previous subsections can be used in parallel to estimate allowable bearing capacity and ensure that settlement beyond a defined limit does not occur. A method of directly comparing stress transmitted to depth with the estimated strength of a snow layer is considered next.

\subsubsection{Comparison of stress with strength}

All of the methods above assume no settlement in estimating an ultimate bearing capacity, or assume a defined amount of settlement to estimate an allowable bearing capacity. However, a preferred method may be to calculate the percentage of stress 
at depth from an applied surface load and then compare this to an estimate for snow strength at that same depth. This allows immediate visual comparison between desired and actual (or estimated) strength. The process to establish such a comparison through the use of CPT data may be as follows:

\section{Estimating vertical stress $\left(\sigma_{v}\right)$}

1. conduct CPT testing at a known rate to perhaps $5 \mathrm{~m}$ depth (vertical pressure bulb from a surface load may extend to $\sim 4 \mathrm{r}$, where $\mathrm{r}$ is the radius of the surface load);

2. determine stratigraphy (layers) from the CPT;

3. estimate an effective elastic modulus $\left(E_{\text {effmacro }}\right)$ for those layers; the value of $E_{\text {effmacro }}$ will affect the percentage of vertical stress transferred;

4. with knowledge of $E_{\text {effmacro }}$ establish the probable stress (from an assumed surface load) within each layer; essentially calculate the dimensions of the pressure bulb, then

\section{Assessing bearing capacity}

5. calculate the strength of each layer affected by the pressure bulb (from averaging CPT data and applying Equation 7.14),

6. choose the layer with the least strength as the critical layer,

7. divide this strength by three (application of a three-fold factor-of-safety, typical for engineering purposes (Dean, 2010)), to then

8. compare this value to the derived vertical stress within the critical layer.

A number of methods of applying such a comparison are now considered. Hirai (2008) in modifying Palmer and Barber's earlier work (Palmer and Barber, 1940) developed an equation for the variation in stress with depth in a semi-infinite soil medium with an equivalent Young's modulus and multi-layers of equivalent thickness as follows:

$$
\Delta \sigma_{z}=q\left[1-\frac{1}{1+{\frac{a^{2}}{z^{2}}}^{\frac{3}{2}}}\right]
$$

where $\Delta \sigma_{z}$ is the vertical stress at depth, $q$ is the uniform surface load, $a$ is the radius of the circular foundation and $z$ is the equivalent layer thickness determined via Palmer and Barber's method (Palmer and Barber, 1940). Assuming a uniform surface load of $100 \mathrm{kPa}$ over a $0.5 \mathrm{~m}$ radius circular foundation, a plot of vertical stress against depth can be plotted with estimated compressive strength (determined from CPT Test 9) versus depth to enable comparison (Figure 8.4): 


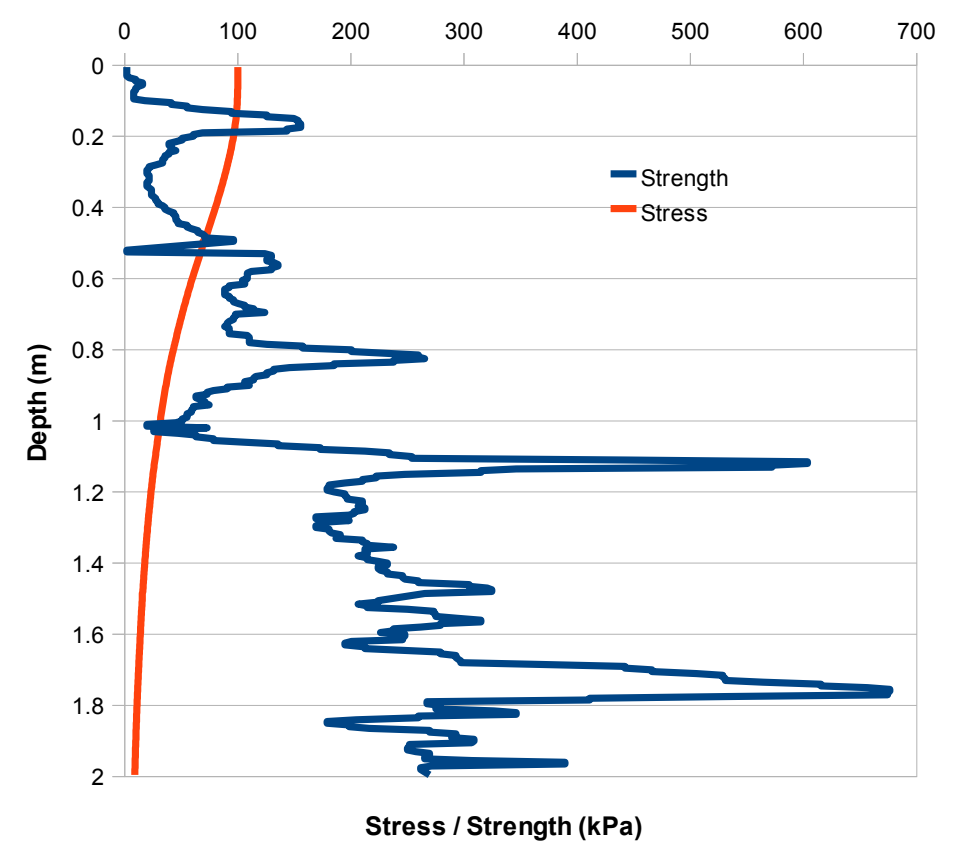

Figure 8.4: Stress from $100 \mathrm{kPa}$ surface load applied over a $0.5 \mathrm{~m}$ radius circular foundation plotted with compressive strength estimated from CPT (Test 9) versus depth.

This compares the decrease in vertical stress with depth, with the snow compressive strength estimated from CPT data. Even allowing for some finite initial distance for the CPT to provide representative strength values, in this example, a deficit in strength is apparent in the upper $0.5 \mathrm{~m}$. If a similar comparison is made with CPT data from a test in compacted snow (Test 59 (Relief Road), Figure 8.5; Test 9 was in natural snow), a similar strength deficit can be observed in the upper $0.14 \mathrm{~m}$, and at two other intervals within the upper metre.

Some of this apparent deficit is because the cone is yet to attain sufficient penetration to provide representative layer strengths. However, as discussed in Chapter 6, this depth is expected to be in the order of $10 \mathrm{~s}$ of $\mathrm{mm}$, hence in this case it appears that some actual strength deficit exists in the upper layers. So, direct comparison of vertical stress due to applied surface loading with strength derived from CPT can provide valuable comparative data.

Bender (1956) also compared penetrative strength data with bearing capacity in testing a processed snow runway in Greenland. He formulated an equation relating the ultimate bearing capacity (BC) in psi and Ramm number (R):

$$
B C=\frac{R}{2}-80
$$

This equation suggests that a $100 \mathrm{kPa}$ circular surface load requires a Ramm number of $>\sim 190$ for sufficient bearing capacity. Via comparisons presented in Chapter 6, this hardness is equivalent to a (non-corrected) CPT tip resistance of $\sim 500 \mathrm{kPa}$ which 


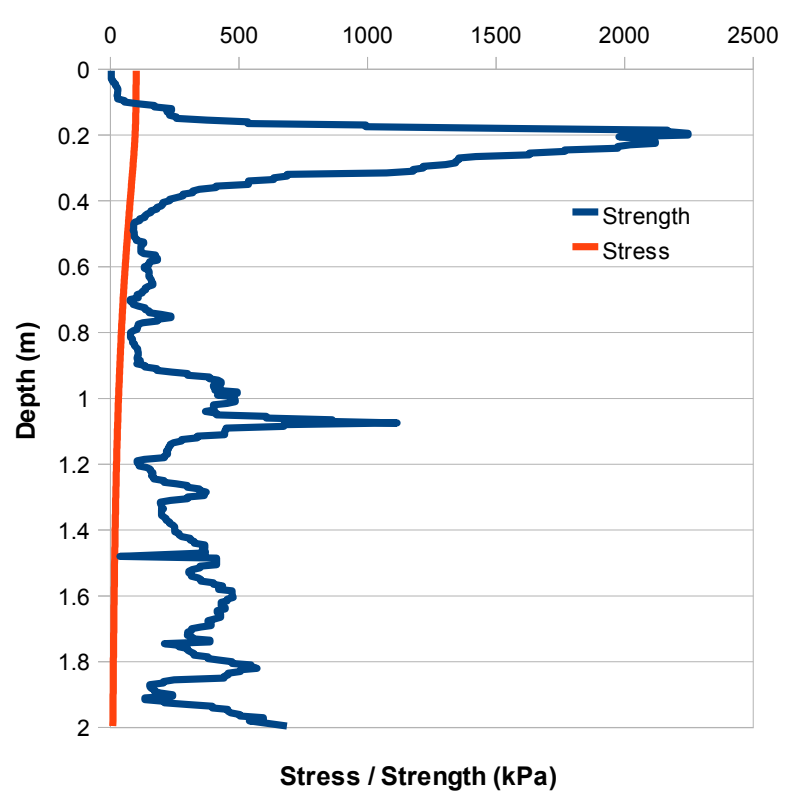

Figure 8.5: Stress from $100 \mathrm{kPa}$ surface load applied over a $0.5 \mathrm{~m}$ radius circular foundation plotted with compressive strength estimated from CPT (Test 59, Relief Road) versus depth.

equates to a compressive strength (or ultimate bearing capacity for the snow) of $\sim$ $150 \mathrm{kPa}$ (assuming density of $450 \mathrm{~kg} \mathrm{~m}^{-3}$ ). This is similar to the applied surface load of $100 \mathrm{kPa}$ used by Bender (1956) and suggests that strength and bearing capacity estimates considered within this work thus far are consistent with historical estimates.

A more efficient method of assessing surface bearing capacity is through the use of FEA or FEM software such as PLAXIS (Plaxis BV, 2010), ABAQUS (Dassault Systmes Simulia Corp., 2010) or Pdisp (Oasys Ltd, 2009).

Marshall (Marshall, 2005) used ABAQUS to simulate surface loading within a layered snowpack, and the FEM package Pdisp (Oasys Ltd, 2009) was used to generate Figure 8.6 which compares an analytical estimate of vertical stress decrease with depth (Hirai, 2008) with that generated via Pdisp (layer data averaged over $20 \mathrm{~cm}$ depth used within Pdisp) for a surface load of $100 \mathrm{kPa}$ applied to a flat circular plate of radius 0.5 $\mathrm{m}$.

Agreement between the analytical and FE methods is very good, and sufficient confidence can be gained in the FEM package to validate its continued application on more elaborate or realistic scenarios. The ability to compare both a strength and an applied surface stress, both derived via CPT was one of the main aims of this research and has been demonstrated here. Although the accuracy of these methods has not yet been proven robustly, a foundation has been established upon which refinements can be made.

Discussion in this chapter so far has been on indirect methods of deriving some measure of bearing capacity from strength or cohesion values derived from CPT in 


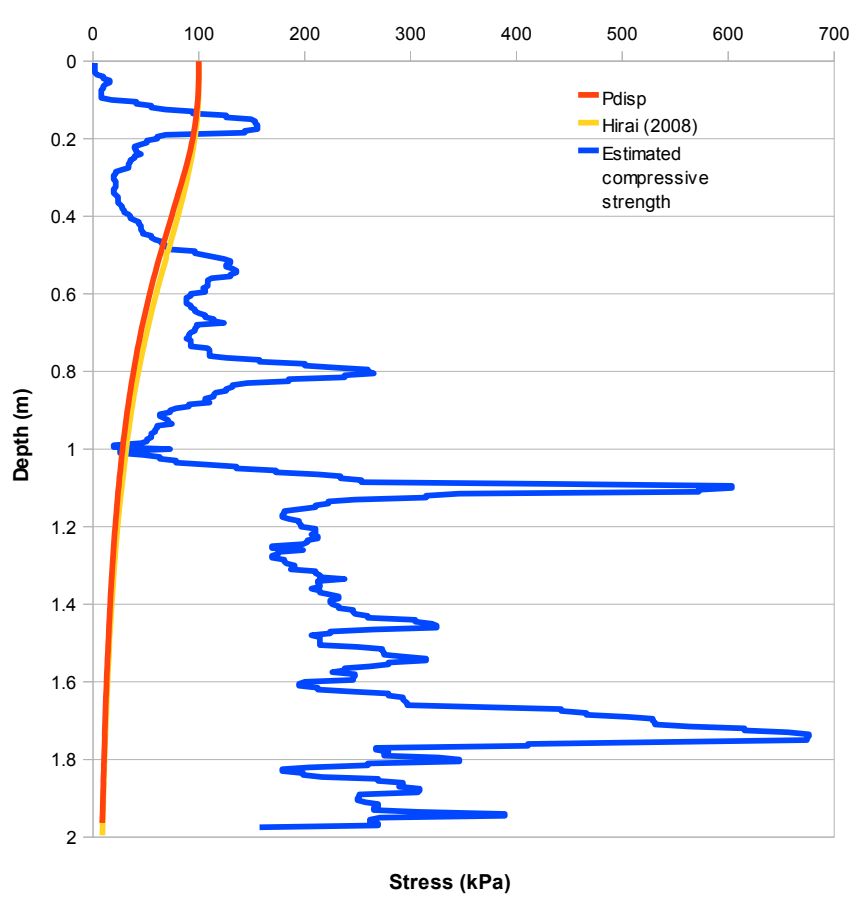

Figure 8.6: Vertical stress with depth generated via FEM (Pdisp) and an analytical method (Hirai, 2008) compared with estimated compressive strength deduced from CPT resistance (Test 9).

snow. Discrepancies exist between the above methods, and a means of assessing bearing capacity directly from $q_{c}$ is desirable. Can bearing capacity be derived directly from CPT resistance data?

\subsection{Direct Assessment of Bearing Capacity}

Methods of directly deducing bearing capacity from CPT were briefly noted in the introduction to this chapter. A number of methods that apply to geomaterials other than snow are considered now.

Schmertmann's method (Schmertmann, 1978) for square footings on sand results in the empirical equation :

$$
q_{u l t}=0.55 \sigma_{a t m}{\frac{q_{t}}{\sigma_{a t m}}}^{0.785}
$$

where $q_{\text {ult }}$ is foundation ultimate bearing capacity, $\sigma_{a t m}=100 \mathrm{kPa}(\sim 1$ tsf in this equation) and $q_{t}$ is cone tip resistance (tsf).

Applying equation 8.11 to typical mean CPT resistance values in snow of perhaps $2 \mathrm{MPa}$ realises an ultimate bearing capacity of $\sim 30 \%$ of measured tip resistance. This is similar to the lower-bound estimate of $30 \%$ for the derivation of base capacity of circular piles from CPT in sand (Jardine et al., 2005).

Application of Meyerhof's theory (Meyerhof, 1976) to sand realises an ultimate 
bearing capacity of $\sim 10 \%$ of depth-averaged tip resistance, Owkati's (in Eslami and Gholami (2006)) realises $\sim 30 \%$, and Tand et al. (1995) produces a value of between $14 \%$ and $22 \%$, notably in cemented sand. Brouwer (2007) simply states that for strip footings on sands, the ultimate surface bearing capacity is less than or equal to tip resistance divided by 30 to 40. A range of values for bearing capacity typically of the order $\sim 10-30 \%$ of cone tip resistance are apparent from these direct empirical methods (Table 8.3).

Table 8.3: Comparison of bearing capacity estimates in sand from CPT via direct methods.

$\begin{array}{lclc}\text { Approach } & \text { Material } & \begin{array}{l}\text { BC as \% of CPT tip } \\ \text { resistance }\end{array} & \text { Remarks } \\ \text { Schmertmann (1978) } & \text { sand } & 30 & \text { circular footing } \\ \text { Jardine et al. (2005) } & \text { sand } & 30 & \text { circular footing } \\ \text { Meyerhof (1976) } & \text { sand } & 10 & \text { circular footing } \\ \text { Owkati } & \text { sand } & 30 & \text { strip footing } \\ \text { Tand et al. (1995) } & \text { cemented sand } & 14-22 & \text { circular footing } \\ \text { Brouwer (2007) } & \text { sand } & 30-40 & \text { strip footing }\end{array}$

Although one of these methods (Jardine et al., 2005) is based on pile base resistance at depth and does not consider surface ejection of material (which would result in decreased capacity) these methods collectively give an estimate for what the ultimate bearing capacity of snow might be as a percentage of CPT tip resistance, especially noting that some cemented material was considered. Further application of these methods to snow should be considered with caution, although application to very cold and dry unsintered snow such as that found at the South Pole may be warranted.

The above discussion briefly considered the direct derivation of bearing capacity from CPT data in geomaterials other than snow, to assess what values might be realised when snow is considered. The direct derivation of bearing capacity from CPT in snow is discussed now.

\subsubsection{Homogeneous snow - allowable bearing capacity}

It appears sensible to try and derive the resistance mobilised by a penetrating cone with the resistance mobilised by a larger static surface load. If the ultimate bearing capacity is surpassed then compaction will occur in both cases and the prime variables in this comparison are shape, size and rate.

Penetrative data from both a cone and a similarly sized flat cone were recorded at Halley, hence a direct empirical relationship between the $60^{\circ}$ cone and a flat plate can be made. Also, the effect of size on penetration resistance has been explored, and appears consistent with measured and modeled values within the literature, hence again a suitable relationship can be established. The variable that may prove the hardest to relate is rate. How does one relate resistance measured during dynamic penetration at 
a rate of $20 \mathrm{~mm} \mathrm{~s}^{-1}$ with a load placed statically on a surface?

A surface load greater than the ultimate bearing capacity will cause the snow to fail in a brittle manner, but what is the strain rate of this process? The speed at which this process will occur depends on the time to failure and the speed to which the load will accelerate under gravity. Assuming that brittle failure will occur almost instantaneously and that the ductile brittle transition for ice is at a penetration rate of $\sim 0.16 \mathrm{~mm} \mathrm{~s}^{-1}$, then all probable bearing capacity failure rates occur in the brittle regime, consistent with $\mathrm{CPT}$ rates. Reinspection of the rate-testing curve derived from CPT data (Figure 5.15) suggests that at higher rates, resistance values will be perhaps $20 \%$ lower than values obtained at the standard CPT rate of $20 \mathrm{~mm} \mathrm{~s}^{-1}$. Therefore, a ratio of expected resistance at CPT rate $\left(20 \mathrm{~mm} \mathrm{~s}^{-1}\right)$ with assumed bearing capacity failure rate $\left(\gg 20 \mathrm{~mm} \mathrm{~s}^{-1}\right)$ can be obtained.

Empirically observed differences in shape, size and rate can now be considered in an attempt to directly estimate the allowable bearing capacity of say a $15 \mathrm{~cm}$ diameter flat plate from CPT tip resistance data:

1. shape - increase resistance derived via cone by $\sim 100 \%$,

2. size - decrease resistance derived via cone by $\sim 20 \%$, and

3. rate - decrease resistance derived via cone by $\sim 20 \%$

Application of these values to a mean tip resistance of $2 \mathrm{MPa}$ results in an estimated ultimate bearing capacity of $\sim 2.5 \mathrm{MPa}$. This is inconsistent with expectations. A review of assumptions is necessary.

Firstly, amplification of cone resistance due to compaction ahead of the cone has not been considered; secondly, neither has the increase in bearing capacity (of perhaps $300 \%$ ) due compaction of the snow under the plate. If the first of these is incorporated then a strength value of $\sim 330 \mathrm{kPa}$ is obtained (from an assumed value of $2 \mathrm{MPa}$ for snow of density $450 \mathrm{~kg} \mathrm{~m}^{-3}$ ) and then by applying the three factors above (due shape, size and rate) an ultimate bearing capacity of $\sim 420 \mathrm{kPa}$ is obtained. If compaction under the plate is then incorporated, an allowable bearing capacity of $\sim 2$ times this value can be obtained: $\sim 840 \mathrm{kPa}$. This value incorporates the estimate that allowable bearing capacity is $\sim 200 \%$ of the ultimate bearing capacity, established earlier, but here the strength value derived from CPT has been manipulated by the shape, size and rate factors, to be directly applied to the $15 \mathrm{~cm}$ diameter flat plate.

This estimate is larger than the direct methods previously considered for sand might suggest (10 to $30 \%$ of cone tip resistance) but this is because of the compaction and resultant increase in stress that occurs in snow and does not occur in sand.

Note that caution needs to be made to ensure that consistency in definition is maintained. Is bearing capacity to be expressed as a percentage of actual measured 
cone tip resistance (e.g. $\mathrm{BC}=25 \%$ of tip resistance) or as an increase on the derived strength of the snow (e.g. $\mathrm{BC}=125 \%$ of deduced snow strength).

This concludes investigation into the direct assessment of bearing capacity from CPT data.

\subsection{Summary}

This chapter examined the application of snow shear strength values derived from CPT data (via the method described in Chapter 7) to predict ultimate and allowable surface bearing capacities for homogeneous and layered media, both indirectly and directly. Table 8.4 summarises approximate surface bearing capacities established via each of these methods, represented as a percentage of mean CPT tip resistance values (for assumed snow density $450 \mathrm{~kg} \mathrm{~m}^{-3}$ ).

Table 8.4: Summary of estimated surface bearing capacity values expressed as percentage of mean CPT tip resistance.

Method

Ultimate homogeneous (Eqn 8.2)

Ultimate homogeneous (Eqn 8.4)

Allowable homogeneous

Ultimate layered

Allowable layered

Allowable homogeneous (direct)

\section{Formula for BC}

$q_{\text {cavg }} / \sim 3$

$q_{\text {cavg }} / \sim 2.2$

$q_{\text {cavg }} / \sim 1.5$

depends on strata depends on strata

$q_{\text {cavg }} / \sim 2.4$
Approximate $\mathrm{BC}(\mathrm{kPa})$ for $q_{\text {cavg }}$ of $2 \mathrm{MPa}$

667

909

1330

$\mathrm{N} / \mathrm{A}$

$\mathrm{N} / \mathrm{A}$

840

This is an example of how CPT data may be applied and is the first attempt to estimate surface bearing capacity both directly and indirectly from CPT data in snow; caution must therefore be used in applying these procedures. Ideally, different methods would be utilised including direct and indirect methods and FEM, and then a conservative decision and an appropriate Factor of Safety would be applied. This work provides a basis for the refinement of procedures to allow more ready application of CPT data for assessing surface bearing capacities in snow and possibly other frozen geomaterials. My research into cone penetration testing in polar snow is concluded in the next chapter. 


\section{Chapter 9}

\section{Conclusion}

This thesis has covered a lot of diverse material, all directed at understanding and applying CPT in polar snow. The assessment of snow strength and surface bearing capacity from CPT data for infrastructure such as roads, runways and buildings has particularly been emphasised. This chapter summarises and concludes my work on $\mathrm{CPT}$ in polar snow.

\subsection{Main aims}

The main aims of this research were to:

1. investigate what manipulation of CPT data is necessary to obtain realistic strength proxy information for snow,

2. assess whether a measure of snow strength can be obtained via CPT, with and without use of additional density data, and

3. determine whether the shallow bearing capacity of snow can be derived both directly and indirectly from CPT data, with and without additional density information.

The addressing of each of these aims is considered below.

From the pilot study in Greenland and the primary CPT in Antarctica, substantial insight was gained into the conduct and interpretation of CPT in polar snow. Specific issues that affect the quantification of CPT resistance data were explored and particularly cone shape, size and penetration rate were observed to significantly effect the magnitude of tip resistance and sleeve friction data. Other effects such as overburden pressure, cone 'sensing' and the formation of a compacted zone were also investigated for their effect on CPT data. However, their impact was not found to be significant within the confines of the testing I conducted.

My work confirmed that many of the factors previously examined by others with regards to $\mathrm{CPT}$ in soil remain pertinent in examining $\mathrm{CPT}$ in polar snow. It also 
confirmed that additional factors such as snow's compressibility, rate-dependence and brittleness (at standard rates of penetration) need to be addressed when deducing quantitative data from CPT in polar snow. Compressibility was seen to have a significant impact on tip resistance, as shown in Chapter 7, and rate effects were seen to significantly influence tip resistance and sleeve friction data; recall Figures 5.15 and 5.17. This result is contrary to that recently confirmed by Floyer and Jamieson (2010) but the differences are probably due to the nature of both the snow and penetrometer used.

I achieved my second aim by deriving an equation based on physical reasoning and empirical evidence (Equation 7.14) that can be used to predict snow shear strength from CPT tip resistance, or vice versa. An estimate of snow shear strength from CPT had not previously been established. At this time, the application of this model is not possible without additional density data. However, I discussed that such data can be estimated from existing densification models and can probably also be ascertained through further rigorous examination of sleeve friction data, thereby negating the need for additional density testing.

Empirical relationships relating values such as Rammsonde hardness and snow shear strength already exist, and rigorous models such as that by Johnson (2003) predict micro and macroscale mechanical parameters for snow, but my contribution is to provide a unique, physically rational method for estimating the shear strength of snow directly from CPT; testing that can be conducted rapidly in the field to depths of $10 \mathrm{~m}$ or more. Further, in deducing snow strength from CPT data the following relationships were suggested, that:

1. snow shear strength can be directly derived from tip resistance $\left(q_{c}\right)$ data,

2. a measure of snow microstructure can be obtained from variation in the friction ratio $\left(R_{f}\right)$, and

3. a measure of snow density can be obtained from variation in sleeve friction $\left(f_{s}\right)$.

In addressing my second aim I have shown that three main determinants of snow's physical behaviour, its strength, density, and microstructure, can potentially all be obtained via one test: the CPT.

The final aim that I addressed in this work was the assessment of surface bearing capacity from CPT data. This application flowed directly from the derivation of strength from CPT data in Chapter 7. It was successfully shown that estimates for both ultimate and allowable surface bearing capacity can be obtained both directly and indirectly, in layered and homogeneous soils, from CPT data. The derivation of bearing capacity directly from CPT has not previously been shown for snow, and although my technique drew from existing methods in soil or snow, not previously has any link between CPT and surface bearing capacity been established for polar snow. 
In summary, my work contributes to the understanding and interpretation of cone penetration testing in polar snow. I presented a rational, physically based model for the derivation of snow strength, discussed the possible extraction of both density and snow microstructure from CPT and I presented methods that may prove valuable in providing rapid estimates for surface bearing capacity in polar snow.

Implications of the contributions I have presented here are far reaching. As the search for natural resources continues unabated into higher latitudes, the requirement to establish infrastructure on frozen or semi-frozen geomaterials will increase. The application of the techniques presented within this thesis may prove of great benefit to both researchers and corporations as the quest to understand and assess the behaviour of frozen geomaterials continues ever more quickly.

\subsection{Additional original contributions}

In addition to addressing my main aims as described above, my thesis work made the following additional contributions:

1. it was the first known use of a penetrometer with a friction sleeve in polar snow. Two types of penetrating cone, both with friction sleeves, were used in Greenland and Antarctica. Friction sleeve data may prove extremely useful in deriving physical parameters for snow from CPT;

2. it described a unique conceptualisation of the forces acting on both the cone and friction sleeve. This conceptualisation was used in addressing one of my primary aims; such a conceptualisation is not previously known to have been applied to snow;

3. direct similarities between snow and other geomaterials such as calcareous sands were established and it was broadly shown that snow exists within a family of structured geomaterials and exhibits behaviour consistent with these geomaterials; and

4. a quantitative relationship between CPT tip resistance values and GPR amplitude variations was quantified, showing that GPR can potentially be used to extrapolate snow mechanical properties derived from CPT over large spatial areas.

\subsection{Limitations of this work}

This was a broad investigation into CPT in polar snow and limitations exist on the application of techniques presented herein. Derivation of strength from CPT relied 
upon limited observations, therefore more rigorous empirical investigation of the relationships proposed is desirable. Variations exist in my presented estimates for surface bearing capacity; ideally large - scale field testing would be conducted in order to empirically verify these relationships. Although CPT resistance variations due to rate were shown to be statistically significant, ideally testing would occur across the ductile - brittle transition to better capture the variation in both tip resistance and sleeve friction across a wider range of both the ductile and brittle regime.

Things that could have been done differently? The Wallingford neutron probe, originally devised for moisture measurement in soil and successfully applied to density measurement in snow by Morris and Cooper (2003) would have been a preferred density measuring device. It can resolve layer density to the cm - scale (Hawley and Morris, 2006) and is simple and efficient to deploy to depths of $10 \mathrm{~m}$ or beyond. It also fortuitously fits almost perfectly within the $\sim 36.7 \mathrm{~mm}$ hole formed during CPT. It was originally envisaged that a neutron probe would be used to complement this research. However, logistical difficulties prohibited its transfer to the Halley site. The pressure sensors used within my testing proved inadequate and ideally a more stable, accurate and precise instrument would be deployed to allow better assessment of vertical stress transfer to depth within a snow pack.

There are limitations to this work, but it is a broad investigation into CPT in polar snow and provides a number of original contributions to such research. It is a valuable resource upon which additional investigations can be based.

\subsection{Further Research}

As is typical of a research project of this nature, there remain many matters that appear worthy of further investigation. Some of these are listed below:

1. seismic cones could be used in conjunction with standard CPT equipment to derive the velocity of $\mathrm{p}$ - and s- waves within the material, thus assisting in the estimation of the dynamic elastic modulus (Lunne et al., 1997). This would assist in estimating vertical stresses under surface loading and the subsequent bearing capacity of the material;

2. a video cone could be used in conjunction with the standard CPT to observe stratigraphy/microstructure. Hawley (2005) has investigated the use of a borehole video camera to measure vertical strain within a snow pack, and a video cone might allow similar interrogation in addition to the assessment of standard CPT parameters;

3. Laser Induced Fluorescence (LIF), an in-situ spectroscopy tool (Dietrich and Leven, 2009) can be used with CPT for the detection of hydrocarbons by fluores- 
cence. Fluorescence intensity is proportional to petroleum hydrocarbon concentration, and a detailed three-dimensional map of the Non-Aqueous Phase Liquid (NAPL) distribution can be generated in real time during spatial testing;

4. use of a nuclear density probe to measure snow density whilst conducting CPT,

5. use of CPT in permafrost. This investigation specifically investigated CPT in polar snow, but much of the interpretation may be pertinent to understanding and assessing the behaviour of frozen/semi-frozen soils;

6. use of a piezocone in non-dry snow (and potentially ice) to aid in interpreting water content. The use of a standard CPT incorporating a piezocone could be used to determine where free-water exists within snow and potentially ice. This may assist in estimating drainage patterns within glaciers and ice sheets;

7. use of a modified or recalibrated piezocone to measure pore air - pressure in snow, firn and ice. The insitu assessment of pore air-pressure is not known to have previously occurred and adaption of existing CPT equipment may make this possible;

8. further work using the cavity expansion method to predict the stress required for compaction after initial fracture could provide valuable modelled data for comparison with field and laboratory testing,

9. non-invasive techniques (GPR) could be further investigated to assess material microstructure and the extent of cohesion/friction within a snow pack, possibly via inducing vibration or excitation/oscillation; and

10. additional investigation into quantitative comparison between GPR amplitude and cone tip resistance would be valuable in validating the spatial extrapolation of CPT data over larger areas.

\subsection{Conclusion}

This thesis has provided an in - depth assessment of cone penetration testing in polar snow. It has made numerous original contributions to the field of snow mechanics and in situ snow investigation and has provided a foundation for the continued development of the techniques and applications outlined within this thesis. The cone penetrometer is an extremely versatile tool upon which many additional sensors can be mounted, often in series, thereby allowing a vast amount of information, visual, chemical, mechanical, seismic etc. all to be obtained in one single test. Its use as a research and investigative tool in polar snow and other frozen or semi-frozen geomaterials appears severely undervalued. 
In his concluding lecture at the 2nd International Conference on Cone Penetration Testing at Huntington Beach, California in May 2010, the renowned soil engineer Professor J. K. Mitchell concluded that the future of cone penetration testing lies in three main areas: the deep ocean, outer space, and the Arctic. The research I have described here perhaps provides some initial steps towards this future..... 


\section{Bibliography}

A. P. van den Berg (2002), "Gonsite Software v1.2", Software.

A. P. van den Berg (2008), GOLOG, A.P. v.d. Berg Machinefabriek B.V., Neerenveen, Netherlands.

Abele, G. (1963), "A Correlation of Unconfined Compressive Strength and Ram Hardness of Processed Snow", Technical Report 85, US Army Cold Regions Research and Engineering Laboratory, Hanover.

Abele, G. (1970), "Deformation of snow under rigid plates at a constant rate of penetration", Research Report 273, US Army Cold Regions Research and Engineering Laboratory, Hanover.

Abele, G. (1990), "Snow Roads and Runways", Technical Report 90-3, US Army Cold Regions Research and Engineering Laboratory, Hanover.

Abele, G. and Gow, A. J. (1976), "Compressibility characteristics of compacted snow", Technical Report 76-21, US Army Cold Regions Research and Engineering Laboratory, Hanover.

Allegre, C. J., Le Mouel, J. L. and Provost, A. (1982), "Scaling rules in rock fracture and possible implications for earthquake prediction", Nature, 297(5861), 47-49.

Alley, R. B. (1987), "Firn Densification by Grain-boundary sliding - A 1st Model", Journal De Physique, 48(C-1), 249-256.

American Avalanche Association (2004), Snow, Weather, and Avalanches: Observational Guidelines for Avalanche Programs in the United States, American Avalanche Association, 1 st ed.

Andersland, O. B. and Ladanyi, B. (1994), An Introduction to Frozen Ground Engineering, Chapman \& Hall Inc., New York.

Arcone, S. A., Spikes, V. B., Hamilton, G. S. and Mayewski, P. A. (2004), "Stratigraphic continuity in $400 \mathrm{MHz}$ short-pulse radar profiles of firn in West Antarctica", Annals of Glaciology, 39, 195. 
Arthern, R., Vaughan, D., Rankin, A., Mulvaney, R. and Thomas, E. (2010), "In situ measurements of Antarctic snow compaction compared with predictions of models", Journal of Geophysical Research F: Earth Surface, 115(3).

Barnes, P., Tabor, D. and Walker, J. C. F. (1971), "The friction and creep of polycrystalline ice.", in: "Proceedings of the Royal Society of London", , vol. A324pp. 127 155 .

Bartelt, P. and von Moos, M. (2000), "Triaxial tests to determine a microstructure-based snow viscosity law", Annals of Glaciology, 31, 457-462.

Bartholomew, I., Nienow, P., Mair, D., Hubbard, A., King, M. A. and Sole, A. (2010), "Seasonal evolution of subglacial drainage and acceleration in a Greenland outlet glacier", Nature Geoscience, 3(6), 408-411.

Bellaire, S., Pielmeier, C., Schneebeli, M. and Schweizer, J. (2009), "Stability algorithm for snow micro-penetrometer measurements", Journal of Glaciology, 55, 805-813.

Bender, J. A. (1956), "Testing of a Compacted Snow Runway", Journal of the Air Transport Division of the American Society of Civil Engineers, 83(AT 1), 20.

Benson, C. S. (1962), "Stratigraphic Studies in the Snow and Firn of the Greenland Ice Sheet", Research Report 70, US Army Snow, Ice and Permafrost Research Establishment, Hanover.

Bergin, A. and Haward, M. (2007), "Frozen assets: Securing Australia's Antarctic future", Technical Report 34, Australian Strategic Policy Institute, Canberra.

Beringen, F. L., Kolk, H. J. and Windle, D. (1982), "Cone penetration and laboratory testing in marine calcareous sediments", Geotechnical Properties, Behaviour and Performance of Calcareous Soils, 170-209.

Bingham, R. G. and Siegert, M. J. (2007), "Radio-Echo Sounding Over Polar Ice Masses", J. Environ. Eng. Geophysics, 12(1), 47-62.

Blackford, J. R. (2007), "Sintering and microstructure of ice: a review", Journal Of Physics D - Applied Physics, 40(21), R355-R385.

Blaisdell, G. L., Lang, R. M., Crist, G., Kurtti, K., Harbin, J. and Flora, D. (1998), "Construction, Maintenance, and Operation of a Glacial Runway, McMurdo Station, Antarctica", Monograph 98-1, US Army Cold Regions Research and Engineering Laboratory, Hanover.

Bolognesi, R. (2007), Snow, Cicerone Press, Milnthorpe, Cumbria, 64 pp.

Bowden, F. P. (1953), "Friction on Snow and Ice", Proceedings of the Royal Society of London. Series A, Mathematical and Physical Sciences, 217(1131), 462-478. 
Brace, W. F., Paulding, B. W. and Scholz, C. (1966), "Dilatancy in the fracture of crystalline rocks", J. Geophys. Res., 71.

Bradley, C. C. (1968), "The resistograph and the compressive strength of snow", Journal of Glaciology, 7(51), $499-506$.

Briaud, J. L. (2001), "Introduction to soil moduli", Geotechnical News.

Brouwer, J. J. M. (2007), "In-situ Soil Testing", Lankelma Limited.

Brown, C. D. (1998), Dictionary of Metallurgy, Wiley.

Brown, R. L. (1979), "A volumetric constitutive law for snow subjected to large strains and strain rates", Technical Report 79-20, US Army Cold Regions Research and Engineering Laboratories, Hanover.

Brown, R. L. and Birkeland, K. W. (1990), "A comparison of the digital resistograph with the ram penetrometer", in: "International Snow Science Workshop", Bigfork, Montana, pp. 19-30.

Budhu, M. (2000), Soil Mechanics $\&$ Foundations, John Wiley \& Sons, Inc., USA.

Buteau, S., Fortier, R. and Allard, M. (2005), "Rate-controlled cone penetration tests in permafrost", Canadian Geotechnical Journal, 42, 184-197.

Casassa, G., Narita, H. and Maeno, N. (1991), "Shear cell experiments of snow and ice friction", Journal of Applied Physics, 69(6), 3745.

Chabot, D. and Kahrl, M. (2009), "SnowPilot", Online.

Chiaia, B. and Frigo, B. (2009), "A scale-invariant model for snow slab avalanches", Journal Of Statistical Mechanics-Theory And Experiment.

Christiano, P. P., Rizzo, P. C. and Jarecki, S. J. (1974), "Compliances of layered elastic systems", in: "Proc Inst Civ Eng (Lond)", , vol. 57pp. 673-683.

Clauset, A., Shalizi, C. and Newman, M. (2009), "Power-law distributions in empirical data", SIAM Review, 51(4), 661-703.

Colbeck, S., Akitaya, E., Armstrong, R., Gubler, H., Lafeuille, J., Lied, K., McClung, D. and Morris, E. M. (1990), "International Classification for Seasonal Snow on the Ground", Tech. rep., Int. Comm. Snow and Ice (IAHS), World Data Center for Glaciology, U. of Colorado, Boulder, CO, USA.

Colbeck, S. C. (1988), "The kinetic friction of snow", Journal of Glaciology, 34(16), $78-86$. 
Colbeck, S. C. (1992), "A Review of the Processes that Control Snow Friction", Technical report, US Army Cold Regions Research and Engineering Laboratory, Hanover.

Colbeck, S. C. (1994), "A review of the friction of snow skis", Journal of Sports Sciences, 12(3), $285-295$.

Colbeck, S. C. (1998), "Sintering in a dry snow cover", Journal Of Applied Physics, 84(8), 4585-4589.

Dassault Systmes Simulia Corp. (2010), "ABAQUS", Software.

Davis, J. R. (ed.) (1998), The Metals Handbook, 2nd ed., ASM International.

De Beer, E. E. (1963), "The scale effect in the transposition of the results of deep-sounding tests on the ultimate bearing capacity of piles and caisson foundations", Geotechnique, 12, p. 39 .

de Quervain, M. (1950), "Die Festigkeitseigenschaften der Schneedecke und ihre Messung", Geofis. Pura Appl., 18.

Dean, E. T. R. (2010), Offshore geotechnical engineering - principles and practice, Thomas Telford Limited, London.

Dietrich, P. and Leven, C. (2009), Groundwater Geophysics, chap. Direct pushtechnologies, Springer Berlin Heidelberg, pp. 347 - 366.

Douglas, B. J. and Olsen, R. S. (1981), "Soil classification using electric cone penetrometer", in: "Cone Penetration Testing and Experience. Proceedings of the ASCE National Convention, St Louis", American Society of Civil Engineers (ASCE), pp. 209-227.

Dowd, T. and Brown, R. L. (1986), "A New Instrument For Determining Strength Profiles In Snow Cover", Journal Of Glaciology, 32(111), 299-301. 0022-1430.

Ericksson, R. (1955), "Friction of runners on snow and ice", Report TL 44, UA Army Snow, Ice and Permafrost Research Establishment.

Eslami, A. and Gholami, M. (2006), "Analytical Model for the Ultimate Bearing Capacity of Foundations from Cone Resistance", Scientia Iranica, 13(3), 223-233.

Evans, K. M. (1987), A Model Study of the End Bearing Capacity of Piles in Layered Calcareous Soils, Ph.D. thesis, University of Oxford.

Faillettaz, J., Louchet, F. and Grasso, J. (2003), "Possible Self-Organised Criticality of snow avalanches.", Confrence plnire au congrs Sud-Amricain de Matriaux 2003 (Jornadas SAM 2003, Congreso Nacional de Metalurgia y Materiales de Chili, Simposio Materia). 
Federolf, P., Fauve, M., Szab, D., Lthi, A., Rhyner, H. U., Schneebeli, M., Ammann, W. and Dual, J. (2004), Snow Engineering V, chap. Mechanical properties of snow during rapid impact, Taylor and Francis Group, London, pp. 209-214.

Fellin, W. and Lackinger, B. (2007), "Foundations of cable car towers upon alpine glaciers", Acta Geotechnica, 2(4), 291. 10.1007/s11440-007-0042-z.

Fierz, C., Armstrong, R. L., Durand, Y., Etchevers, P., Greene, E., McClung, D. M., Nishimura, K., Satyawali, P. K. and Sokratov, S. A. (2009), "The International Classification for Seasonal Snow on the Ground", IHP-VII Technical Documents in Hydrology 83, UNESCO-IHP, Paris. IACS Contribution No. 1.

Floyer, A. J. (2006), "An update on digital penetrometer technology", University of Calgary, Applied Snow and Avalance Research.

Floyer, A. J. and Jamieson, J. B. (2006), "Empirical analysis of snow deformation below penetrometer tips", in: "International Snow Science Workshop", Telluride, pp. 555561.

Floyer, J. (2008), Layer detection and snowpack stratigraphy characterisation from digital penetrometer signals, Ph.D. thesis, University of Calgary.

Floyer, J. A. and Jamieson, J. B. (2010), "Rate-effect experiments on round-tipped penetrometer insertion into uniform snow.", Journal of Glaciology, 56(198), 664-672.

Fogarty, E. (2011), "Antarctica: Assessing and Protecting Australia's National Interests", Tech. rep., Lowy Institute for International Policy.

Frenkel, D. (2010), "The tetrahedral dice are cast and pack densely", Physics, 3(37).

Fukue, M. (1977), Mechanical Performance of Snow Under Loading, Ph.D. thesis, McGill University, Montreal.

Gameda, S., Vigneault, C. and Vijaya Raghavan, G. S. (1996), "Snow behaviour under compaction for the production of ice", Energy, 21(1), 15.

Geduhn, M. and Enss, D. (2006), "Bearing capacity of 20 single column shallow foundations of the Amtarctic Station Neumayer III over 30 years service life", in: "The 25th International Conference on Offshore Mechanics and Arctic Engineering OMAE, CCH Hamburg", American Society of Mechanical Engineers, Hamburg.

Geophysical Survey Systems Inc. (2006), SIR System-3000 User's Manual, Salem, New Hampshire, revision $\mathrm{f}$ ed.

Geophysical Survey Systems Inc. (2007), RADAN 6.5 User's Manual, Salem, New Hampshire, revision d ed. 
Gibson, L. J. and Ashby, M. F. (1997), Cellular Solids - Structure and properties, 2nd ed., Cambridge University Press.

Gill, W. R. (1968), "Influence of compaction hardening of soil on penetration resistance", Trans. Am. Soc. Agric. Eng., 11, 741-745.

Gleason, J. A. (2004), "Particle Image Velocimetry; A New Technique to Measure Strain in Loaded Snow", Colorado Geological Survey.

Goodwin, I. (1991), "Snow-accumulation variability from seasonal surface observations and firn-core stratigraphy, eastern Wilkes Land, Antarctica", Journal of Glaciology, $37(127)$.

Griffith, A. A. (1921), "The Phenomena of Rupture and Flow in Solids", Philosophical Transactions of the Royal Society of London. Series A, Containing Papers of a Mathematical or Physical Character, 221(582-593), 163-198.

Gubler, H. (1978), "Determination of the mean number of bonds per snow grain and of the dependence of the tensile strength of snow on stereological parameters", Journal of Glaciology, 20(83), 329-341.

Gubler, H. U. (1975), "On the rammsonde hardness equation", in: "Grindelwald Symposium on snow mechanics", No. 114 in IAHS Publ., pp. 111-121.

Haefeli, R. (1936), "Beitrge zur Gologie der Schweiz Geotechn.", Serie-Hydr, 3.

Haegeli, P. and McClung, D. M. (2003), "Avalanche characteristics of a transitional snow climate-Columbia Mountains, British Columbia, Canada", Cold Regions Science and Technology, 37(3), 255-276.

Haehnel, R. B., Shoop, S. A. and Pw (2004), "A macroscale model for low density snow subjected to rapid loading", Cold Regions Science and Technology, 40(3), 193-211. Dec 0165-232X Article A macroscale model for low density snow subjected to rapid loading ISI:000225802700004 37 ELSEVIER SCIENCE BV English.

Hajiabdolmajid, V., Kaiser, P. K. and Martin, C. D. (2002), "Modelling brittle failure of rock", International Journal of Rock Mechanics and Mining Sciences, 39(6), 731.

Hawley, R. L. (2005), Borehole Investigations of Firn Processes, Ph.D. thesis, University of Washington.

Hawley, R. L. and Morris, E. M. (2006), "Borehole optical stratigraphy and neutronscattering density measurements at Summit, Greenland", Journal of Glaciology, 52(179), 491-496.

Herron, M. M. and Langway, C. C. (1980), "Firn Densification - An empirical model", Journal of Glaciology, 25(93), 373-385. 
Hirai, H. (2008), "Settlements and stresses of multi-layered grounds and improved grounds by equivalent elastic method", International Journal for Numerical and Analytical Methods in Geomechanics, 32, 523-557.

Hoek, E. and Brown, E. (1997), "Practical estimates of rock mass strength", International journal of rock mechanics and mining sciences, 34(8), 1165. 1365-1609.

Holler, P. and Fromm, R. (2010), "Quantification of the hand hardness test", Annals of Glaciology, 51(54).

Hooke, R. L. (2005), Principles of Glacier Mechanics.

Irwin, G. J., Mohamed, A. M. O., Alammawi, S. and Yong, R. N. (1991), "Prediction of load carrying capacity of deep snow by rammsonde hardness", Journal of Terramechanics, 28(2-3), 167.

Jaksa, M. B., Kaggwa, W. S. and Brooker, P. I. (2002), "An Improved Statistically Based Technique for Evaluating the CPT Friction Ratio", Geotechnical Testing Journal, 25(1), 61-69.

Jamieson, B. and Johnston, C. D. (2001), "Evaluation of the shear frame test for weak snowpack layers", Annals of Glaciology, 32, 59-69.

Janbu, N., Bjerrum, L. and Kjaernsli, B. (1956), "Soil mechanics applied to some engineering problems.", Norwegian Geotech. Inst. Publ., 16, 30-32.

Jardine, R., Chow, F., Overy, R. and Standing, J. (2005), ICP design methods for driven piles in sands and clays, Thomas Telford Ltd.

Johnson, J. and Schneebeli, M. (1998), "Snow Strength Penetrometer", .

Johnson, J. B. (2003), "A Statistical Micromechanical Theory of Cone Penetration in Granular Materials", Technical Report ERDC/CRREL TR-03-3, Cold Regions Research and Engineering Laboratory, Hanover.

Johnson, J. B. and Schneebeli, M. (1999), "Characterizing the microstructural and micromechanical properties of snow", Cold Regions Science and Technology, 30(1-3), 91.

Jun, L. and Zwally, H. J. (2002), "Modeled seasonal variations of firn density induced by steady-state surface air-temperature cycle", Annals of Glaciology, 34, 299 - 302.

Kaempfer, T. U. and Schneebeli, M. (2007), "Observation of isothermal metamorphism of new snow and interpretation as a sintering process", Journal Of Geophysical ResearchAtmospheres, 112(D24). 0148-0227 D24101. 
Kartashov, S. N. (1965), "Mechanical properties of snow and firn", in: "Symposium international sur les aspects scientifiques des avalanches de neige", 69, Union de Geodesie et Geophysique internationale, Association Internationale d'Hydrologie Scientifique, p. 424.

King, R. W., van Hooydonk, W. R., Kolk, H. F. and Windle, D. (1980), "Geotechnical investigations of calcareous soils on the North West Shelf, Australia", Offshore Technology Conference.

Kinosita, S. (1964), "Intrusion of a rigid cone into snow", Series A 23, The Institute of Low Temperature Science, Hokkaido University, Sapporo, Japan. In Japanese with English summary.

Kinosita, S. (1967), "Compression of snow at constant speed", in: "International Conference on Physics of Snow and Ice", Hokkaido University, Japan.

Kirchner, H. O. K. (2001), "Brittle fracture of snow", Physical Aspects of Fracture. Proceedings of the NATO Advanced Study Institute on Physical Aspects of Fracture, 47-57.

Kirchner, H. O. K., Michot, G., Narita, H. and Suzuki, T. (2001), "Snow as a foam of ice: plasticity, fracture and the brittle-to-ductile transition", Philosophical Magazine A, 81, 2161. Doi:10.1080/01418610010029043.

Kirchner, H. O. K., Peterlik, H., Michot, G. and Wc (2004), "Size independence of the strength of snow", Physical Review E, 69(1), 8.

Klein, G. J. (1947), "The snow characteristics of aircraft skis", Aeronautical Report AR-2, National Research Council of Canada.

Kondratyeva, A. S. (1945), "Increase of density of snow under compressive loading", The Physico-Mechanical Properties of Snow and their Application in the Construction of Airfields and Roads. Translated by Bureau of Yards and Docks, 1949.

Kovacs, A., Gow, A. J. and Morey, R. M. (1995), "The in-situ dielectric constant of polar firn revisited", Cold Regions Science and Technology, 23(3), 245.

Kronholm, K. (2004), Spatial Variability of Snow Mechanical Properties with regards to avalanche formation, Ph.D. thesis, University of Zurich, Zurich.

Kubo, T., Durham, W. B., Stern, L. A. and Kirby, S. H. (2006), "Grain Size-Sensitive Creep in Ice II", Science, 311(5765), 1267-1269.

Ladanyi, B. (1981), "Mechanical behaviour of frozen soils", in: "Proceedings of the International Symposium on Mechanical Behaviour of Structured Media", , vol. BCarleton University, Elsevier, New York., Ottawa, pp. 205-245. 
Ladanyi, B. (1982), "Determination of geotechnical parameters of frozen soils by means of the cone penetration test", in: "Proceedings of the Second European Symposium on Penetration Testing", Amsterdam, pp. 671-678.

Ladanyi, B. (1985), "Use of the Cone Penetration Test for the Design of Piles in Permafrost", Journal of Energy Resources Technology, 107, 183-187.

Ladanyi, B., Lunne, T. and Winsor, W. (1991a), "Experience with the performance of load-controlled cone penetration tests in permafrost and ice.", in: "Proceedings of the 44th Canadian Geotechnical Conference, Calgary", , vol. 41pp. 41-141-8.

Ladanyi, B. and Paquin, J. (1978), "Creep behavior of frozen sand under a deep circular load", in: "Proceedings of the Third International Conference on Permafrost", , vol. 1Edmonton, pp. 679-686.

Ladanyi, B., Steel, A., Winsor, W. D. and Clark, J. I. (1991b), "Comparison of three methods for in situ ice strength determination", in: "Eleventh International Conference on Port and Ocean Engineering under Arctic Condition", St. John's, pp. 344-365.

Lang, R. M., Blaisdell, G. L., Durso, C., Reinemer, G. and Lesher, M. (1997), "Processing snow for high strength roads and runways", Cold Regions Science and Technology, 25(1), 17-31.

Lee, J. H. and Huang, D. (2010), "Material point method modeling of porous semi-brittle materials", .

Lee, J. H. and Wang, W. (2009), "Characterization of snow cover using ground penetrating radar for vehicle trafficability - Experiments and modeling", Journal Of Terramechanics, 46(4), 189-202. ISI Document Delivery No.: 491NE Times Cited: 0 Cited Reference Count: 28 English Proceedings Paper 0022-4898 Sp. Iss. SI.

Lee, S. M., Haas, W. M. and Wuori, A. F. (1988), "Improved Techniques for Construction of Snow Roads and Airstrips", Technical report, US Army Cold Regions Research and Engineering Laboratory, Hanover.

Leite, M. H. and Ferland, F. (2001), "Determination of unconfined compressive strength and Young's modulus of porous materials by indentation tests", Engineering Geology, $\mathbf{5 9}(3-4), 267$.

Leroueil, S. and Vaughan, P. R. (1990), "The general and congruent effects of structure in natural soils and weak rocks", Geotechnique, 41(3), 467 - 488.

Lu, Q., Randolph, M., Hu, Y. and Bugarski, I. (2004), "A numerical study of cone penetration in clay", Geotechnique, 54(4), 257-267. 
Lunne, T., Robertson, P. K. and Powell, J. J. M. (1997), Cone Penetration Testing in Geotechnical Practice, Blackie Academic \& Professional, London.

Lutz, E., Birkeland, K. W., Kronholm, K., Hansen, K. and Aspinall, R. (2007), "Surface hoar characteristics derived from a snow micropenetrometer using moving window statistical operations", Cold Regions Science And Technology, 47(1-2), 118-133.

MacDowall, J. (1964), "Glaciological Observations", in: Brunt, D. (ed.), "The Royal Society International Geophysical Year Antarctic Expedition Halley Bay, Coats Land, Falkland Islands Dependencies, 1955 - 59.", , vol. IVThe Royal Society, London.

Mackenzie, R. and Payten, W. (2002), "A Portable, Variable-Speed, Penetrometer for Snow Pit Evaluation", in: "International Snow Science Workshop", Penticton, p. 7.

Marshall, H. P. (2005), Snowpack spatial variability: towards understanding its effect on remote sensing measurements and snow slope stability, Ph.D. thesis, University of Washington.

Marshall, H. P. and Johnson, J. B. (2009), "Accurate inversion of high-resolution snow penetrometer signals for microstructural and micromechanical properties", J. Geophys. Res., 114(F4).

Marshall, H. P., Schneebeli, M. and Koh, G. (2007), "Snow stratigraphy measurements with high-frequency FMCW radar: Comparison with snow micro-penetrometer", Cold Regions Science and Technology, 47(1-2), 108.

Martin, C. D. (1997), "The effect of cohesion loss and stress path on brittle rock strength", Canadian Geotechnical Journal, 34, 698 - 725.

McCallum, A. B. (2006), "The Movement and Expected Lifetime of the Casey Ice Runway", ASCE.

McClung, D. and Schaerer, P. (1993), The Avalanche Handbook, The Mountaineers, Seattle, Washington.

McClung, D. M. (1979), "In-situ estimates of the tensile strength of snow utilizing large sample sizes", Journal of Glaciology, 22(87), 321-329.

McClung, D. M. (1981), "Fracture Mechanical Models of Dry Slab Avalanche Release", J. Geophys. Res., 86.

McClung, D. M. (2005), "Dry slab avalanche shear fracture properties from field measurements.", Journal of Geophysical Research - Earth Surface, 110(F4).

Mellor, M. (1964), "Properties of Snow", Monograph III-A1, US Army Cold Regions Reearch and Engineering Laboratory, Hanover. 
Mellor, M. (1966), Appl. Mech. Rev., 19, 379-389.

Mellor, M. (1972), "Normalization of specific energy values", International Journal of Rock Mechanics and Mining Science \& Geomechanics Abstracts, 9(5), 661.

Mellor, M. (1975), "A Review of Basic Snow Mechanics", in: "International Symposium on Snow Mechanics", , vol. International Association of Hydrological Sciences Publication 114International Association of Hydrological Sciences, Grindewald, pp. 251-291.

Mellor, M. (1993), "Notes on Antarctic Aviation", Technical Report 93-14, US Army Cold Regions Research and Engineering Laboratory, Hanover.

Meyerhof, G. (1951), "The ultimate bearing capacity of foundations.", Geotechnique, 2(4), 301-332.

Meyerhof, G. G. (1948), "An investigation of the bearing capacity of shallow footings on dry sand", in: "Proceedings of the 2nd International Conference on Soil Mechanics and Foundation Engineering", Rotterdam.

Meyerhof, G. G. (1976), "Bearing capacity and settlement of pile foundations", Journal of the Geotechnical Engineering Division, ASCE, 102 (GT3), 197-228. 11th Terzaghi Lecture.

Morris, E. M. and Cooper, J. D. (2003), "Instruments and methods - Density measurements in ice boreholes using neutron scattering", Journal Of Glaciology, 49(167), 599-604.

Mulqueen, J., Stafford, J. V. and Tanner, D. W. (1977), "Evaluation Of Penetrometers For Measuring Soil Strength", Journal Of Terramechanics, 14(3), 137-151.

Muro, T. and O'Brien, J. (2004), Terramechanics, A. A. Balkema.

Nakamura, T., Abe, O., Hashimoto, R. and Ohta, T. (2010), "A dynamic method to measure the shear strength of snow", Journal of Glaciology, 56, 333-338.

Newbolt, H. J. (1892), "Vita Lampada", Poem.

Ng, I. T. and Zhou, C. Y. (2010), "Uncertainty-Based Optimization of Site Characterization using CPT", in: "Proceedings, 2nd International Symposium on Cone Penetration Testing, Huntington Beach, CA, USA, May 2010", .

Nicot, F. (2004), "Constitutive modelling of snow as a cohesive-granular material", Granular Matter, 6(1), 47-60.

Nowatzki, E. A. and Karafiath, L. L. (1972), "Effect of cone angle on penetration resistance", in: "Highway Research Record", 405, Highway Research Board, Washington, DC, pp. 51-59. 
Oasys Ltd (2009), "Pdisp Version 19.0", Software.

Palmer, L. A. and Barber, E. S. (1940), "Soil displacement under a loaded circular area", in: "Proc. High. Res. Board", , vol. 20pp. 279-286.

Paterson, W. (1994), The Physics of Glaciers, 3rd ed., Elsevier Science Ltd., Oxford.

Perla, R., Beck, T. and Cheng, T. (1982), "The shear strength index of alpine snow", Cold Regions Science and Technology, 6(1), 11-20.

Perla, R. and Glenne, B. (1981), Handbook of snow; principles, processes, management and use., chap. Skiing, Pergamon Press, Toronto etc., pp. 709-40.

Petrovic, J. J. (2003), "Mechanical properties of ice and snow", Journal of Materials Science, 38, 1-5.

Pielmeier, C. and Schneebeli, M. (2003), "Stratigraphy and changes in hardness of snow measured by hand, ramsonde and snow micro penetrometer: a comparison with planar sections", Cold Regions Science And Technology, 37(3), 393-405.

Plaxis BV (2010), "PLAXIS", Software.

Poulos, H. G. and Davis, E. H. (1974), Elastic Solutions for Soil and Rock Mechanics, John Wiley \& Sons, Inc.

Press, W. H., Teukolsky, S. A., Vetterling, W. T. and Flannery, B. P. (1992), Numerical Recipes in C: The Art of Scientific Computing, Cambridge University Press, Cambridge, England.

Pytka, J. (2009), "Determination of snow stresses under vehicle loads", Cold Regions Science and Technology, 60, 137-145. 0165-232X doi: DOI: 10.1016/j.coldregions.2009.10.002.

Ramsey, N. (2002), "A Calibrated Model For The Interpretation Of Cone Penetration Tests (CPTs) In North Sea Quaternary Soils", in: "Offshore Site Investigation and Geotechnics 'Diversity and Sustainability'; Proceedings of an International Conference, November 26 - 28, 2002, London, UK", .

Rees, W. G. (2006), Remote Sensing of Snow and Ice, Taylor and Francis.

Renshaw, C. E. and Schulson, E. M. (2001), "Universal behaviour in compressive failure of brittle materials", Nature, 412(6850), 897-900.

Rick, U. and Albert, M. (2004), "Microstructure of West Antarctic Firn and Its Effect on Air permeability", Technical Report 04-16, US Army Cold Regions Research and Engineering Laboratory, Hanover. 
Robertson, P. K. (1990), "Soil classification using the cone penetration test", Canadian Geotechnical Journal, 27(1), 151-158.

Robertson, P. K. (1991), "Estimation of foundation settlements in sand from CPT", in: "ASCE Geotechnical Engineering Congress", Boulder, CO.

Robertson, P. K. and Cabal, K. L. (2010), "Guide to cone penetration testing for geotechnical engineering", Spiral bound booklet, Gregg Drilling \& Testing Inc., Signal Hill, California.

Robertson, P. K., Sasitharan, S., Cunning, J. C. and Sego, D. (1995), "Shear-wave velocity to evaluate in-situ state of Ottawa sand", .

Rogers, J. D. (2006), "Subsurface Exploration Using the Standard Penetration Test and the Cone Penetrometer Test", Environmental and Engineering Geoscience, 12(2), 161179.

Russell-Head, D. S. and Budd, W. F. (1989), "Compacted-snow runways: Guidelines for their design and construction in Antarctica", Tech. Rep. 89-10, US Army Cold Regions Research and Engineering Laboratories, Hanover.

Sammonds, P. R. (2005), "Deformation dynamics: Plasticity goes supercriticial", Nat Mater, 4(6), 425-426.

Sammonds, P. R. (2006), "Planetary Science: Creep and Flow on the Icy Moons of the Outer Planets", Science, 311(5765), 1250-1251.

Scapozza, C. and Bartelt, P. (2003a), "Triaxial tests on snow at low strain rate. Part II. Constitutive behaviour", Journal Of Glaciology, 49(164), 91-101.

Scapozza, C. and Bartelt, P. A. (2003b), "The influence of temperature on the smallstrain viscous deformation mechanics of snow: a comparison with polycrystalline ice", in: "Annals Of Glaciology, Vol 37", , vol. 37 of Annals Of Glaciologypp. 90-96.

Schaap, L. H. J. and Fohn, P. M. B. (1987), "Cone Penetration Testing In Snow", Canadian Geotechnical Journal, 24(3), 335-341.

Schmertmann, J. H. (1978), "Guidelines for cone penetration test, performance and design", Fhwa-ts-78-209, US Federal Highway Administration.

Schneebeli, M. and Johnson, J. B. (1998), "A constant-speed penetrometer for highresolution snow stratigraphy", Annals of Glaciology, 26, 107-111.

Schneebeli, M., Pielmeier, C. and Johnson, J. B. (1999), "Measuring snow microstructure and hardness using a high resolution penetrometer", Cold Regions Science and Technology, 30(1-3), 101. 
Schofield (1998), "The "Mohr-Coulomb" Error", Technical Report 305, Cambridge University Engineering Department, Division D Soil Mechanics Group.

Schulson, E. M. (2001), "Brittle failure of ice", Engineering Fracture Mechanics, 68(1718), 1839.

Schulson, E. M. and Duval, P. (2009), Creep and fracture of ice, Cambridge University Press, Cambridge, UK.

Schweizer, J. (1999), "Review of dry snow slab avalanche release", Cold Regions Science and Technology, 30(1-3), 43.

Schweizer, J., Heilig, A., Bellaire, S. and Fierz, C. (2008a), "Variations in snow surface properties at the snowpack-depth, the slope and the basin scale", Journal Of Glaciology, 54(188), 846-856.

Schweizer, J., Kronholm, K., Jamieson, J. B. and Birkeland, K. W. (2008b), "Review of spatial variability of snowpack properties and its importance for avalanche formation", Cold Regions Science and Technology, 51(2-3), 253.

Schweizer, J., Michot, G. and Kirchner, H. (2004), "On the fracture toughness of snow", Annals of Glaciology, 38, 1-8.

Shapiro, L. H., Johnson, J. B., Sturm, M. and Blaisdell, G. L. (1997), "Snow Mechanics. Review of the State of Knowledge and Applications", Report 97-3, US Army Cold Regions Research and Engineering Laboratory, Hanover.

Shimbo, M. (1972), "Friction on snow of ski soles unwaxed and waxed", Scientific Study of Skiing in Japan.

Shoop, S. A. (1993), "Terrain Characterization for Trafficability", Report 6, US Army Cold Regions Research and Engineering Laboratories, Hanover, New Hampshire.

Shoop, S. A. and Alger, R. (1998), "Snow deformation beneath a vertically loaded plate formation of pressure bulb with a limited lateral displacement", in: "Ninth International conference on Cold Regions Engineering", American Society of Civil Engineers, Duluth, pp. $143-150$.

Sigrist, C. (2006), Measurement of Fracture Mechanical Properties of Snow and Application to Dry Snow Avalanche Release, Ph.D. thesis, Swiss Federal Institute of Technology, Zurich.

Sommerfeld, R. A. (1974), "A Weibull Prediction of the Tensile Strength-Volume Relationship of Snow", Journal of Geophysical Research, 79(23), 3353 - 3356.

Stehle, N. (1970), "Snow compaction - Investigation of metamorphism of snow", Technical Report R-706, Naval Civil Engineering Laboratories, Port Hueneme. 
Sturm, M. and Benson, C. (2004), "Scales of spatial heterogeneity for perennial and seasonal snow layers", Annals of Glaciology, 38, 253.

Sturm, M., Johnson, J. and Holmgren, J. (2004), "Variations in the mechanical properties of arctic and subarctic snow at local (1-m) to regional (100-km) scales", in: "International Symposium on Snow Monitoring and Avalanches (ISSM-2004) Manali, India",

Swithinbank, C. (1969), "Airborne Radio Echo Sounding by the British Antarctic Survey", The Geographical Journal, 135(4), 551-553.

Szabo, D. and Schneebeli, M. (2007), "Subsecond sintering of ice", Applied Physics Letters, $\mathbf{9 0}(15), 151,916$.

Tancret, F. and Osterstock, F. (2003), "Modelling the toughness of porous sintered glass beads with various fracture mechanisms", Philosophical Magazine, 83(1), 137 - 150.

Tand, K. E., Funegard, E. G. and Warden, P. E. (1995), "Predicted/measured bearing capacity of shallow footings on sand", in: "Proceedings of the International Symposium on Cone Penetration Testing, CPT '95, Linkoping, Sweden", , vol. 2Swedish Geotechnical Society, pp. 589-594.

Tarr, M. (2011), "Stress and its effect on materials", Online.

Teh, K. L., Cassidy, M. J., Leung, C. F., Chow, Y. K., Randolph, M. F. and Quah, C. K. (2008), "Revealing the bearing capacity mechanisms of a penetrating spudcan through sand overlying clay", Geotechnique, 58(10), 793-804.

Terzaghi, K. (1943), Theoretical Soil Mechanics, Wiley, New York.

Tharp, T. M. (1983), "Analogies between the high-temperature deformation of polyphase rocks and the mechanical behavior of porous powder metal", Tectonophysics, 96(3-4), $\mathrm{T} 1$.

Turner, R., Schmidt, K., Fornier, M. and Lepage, K. (2001), "Development of a Simple System for Measuring Peak Soil Stresses", in: "Agricultural Equipment Technology Conference", American Sociey of Agricultural Engineers.

Upadhyay, A., Joshi, S. K. and Chandel, C. (2007), "Tensile Strength of Snow using Centrifugal Technique", Defence Science Journal (India), 57(6).

Vreugdenhil, R., Davis, R. and Berrill, J. (1994), "Interpretation of cone penetration results in multilayered soils", Int. J. Numer. Anal. Meth. Geomech., 18(9), 585-599.

Weibull, W. (1939a), "The Phenomenon of Rupture in Solids", p. 55.

Weibull, W. (1939b), "A Statistical Theory of the Strength of Materials", p. 45. 
Whiteley, G. M. and Dexter, A. R. (1981), "The dependence of soil penetrometer pressure on penetrometer size", Journal of Agricultural Engineering Research, 26(6), 467.

Whitlow, R. (2001), Basic Soil Mechanics, 4th ed., Pearson Education Limited, Harlow.

Wuori, A. F. (1962), "Supporting capacity of processed snow runways", Technical Report 82, Cold Regions Research and Engineering Laboratories, Hanover.

Yosida, Z. (1963), "Physical properties of snow", in: Kingery, W. D. (ed.), "Ice and Snow; Properties, Processes and Applications", M.I.T. Press, Cambridge, Massachusetts.

Yu, H. S. and Mitchell, J. K. (1998), "Analysis of cone resistance: Review of methods", Journal of Geotechnical and Geoenvironmental Engineering, 140-149.

Yuanlin, Z., Ping, H., Jiayi, J. and Jianming, Z. (1998), "Effect of temperature and strain rate on the constitutive relation of frozen saturated silt", in: "Proceedings of the 7th International Permafrost Conference", Yellowknife, Canada. 
Appendix A

Summary table of Antarctic CPT tests 


\begin{tabular}{|c|c|c|c|c|c|}
\hline Test & Date & Locn & $\sim$ Rate & Cone & Remarks \\
\hline 1 & $21 / 01 / 10$ & $\begin{array}{l}\text { Vehicle } \\
\text { Park }\end{array}$ & $\begin{array}{c}38- \\
40 \mathrm{~mm} / \mathrm{sec}\end{array}$ & standard & Lots of vibration $>2.5 \mathrm{~m}$ \\
\hline 2 & $21 / 01 / 10$ & $\begin{array}{l}\text { Vehicle } \\
\text { Park }\end{array}$ & $\begin{array}{c}38- \\
40 \mathrm{~mm} / \mathrm{sec}\end{array}$ & standard & $\begin{array}{c}\text { Lots of rod slip. } \sim 3-4 \text { false pushes for } \\
\text { maybe 20cm each time. }\end{array}$ \\
\hline 3 & $21 / 01 / 10$ & $\begin{array}{l}\text { Vehicle } \\
\text { Park }\end{array}$ & $\begin{array}{c}38- \\
40 \mathrm{~mm} / \mathrm{sec}\end{array}$ & standard & $\begin{array}{l}\text { Depth encoder stops when too much } \\
\text { vibration \& then catches up. }\end{array}$ \\
\hline 4 & $21 / 01 / 10$ & $\begin{array}{l}\text { Vehicle } \\
\text { Park }\end{array}$ & $\begin{array}{c}38- \\
40 \mathrm{~mm} / \mathrm{sec}\end{array}$ & standard & $\begin{array}{l}\text { Couple of rod slips \& vibration hence } \\
\text { DE stopping. }\end{array}$ \\
\hline 5 & $21 / 01 / 10$ & $\begin{array}{l}\text { Vehicle } \\
\text { Park }\end{array}$ & $\begin{array}{c}38- \\
40 \mathrm{~mm} / \mathrm{sec}\end{array}$ & standard & No rod slip - some vibration. \\
\hline 6 & $21 / 01 / 10$ & $\begin{array}{l}\text { Vehicle } \\
\text { Park }\end{array}$ & $\begin{array}{c}38- \\
40 \mathrm{~mm} / \mathrm{sec}\end{array}$ & Flat plate & \\
\hline 7 & $21 / 01 / 10$ & $\begin{array}{l}\text { Vehicle } \\
\text { Park }\end{array}$ & $\begin{array}{l}20- \\
21 \mathrm{~mm} / \mathrm{sec} \\
\text { (turned- } \\
\text { down by } 1 / 4 \\
\text { turn) }\end{array}$ & Flat plate & $\begin{array}{l}\text { Test terminated early @ 2.6m due } \\
\text { reaching 30MPa. Also overwrote } \\
\text { some data as left DE on whilst } \\
\text { retrieving initial } 20 \mathrm{~cm} \text { or so. Cone of } \\
\text { very hard ice formed on flat plate - } \\
\text { see photo. }\end{array}$ \\
\hline 8 & $21 / 01 / 10$ & & $\begin{array}{c}20- \\
21 \mathrm{~mm} / \mathrm{sec}\end{array}$ & standard & Compare all with GPR run too. \\
\hline 9 & $22 / 01 / 10$ & $\begin{array}{l}\text { Test Site } \\
\text { (Virgin } \\
\text { snow) }\end{array}$ & 25 & standard & $\begin{array}{l}\text { For location see photo. } \\
\text { Initial observation suggests friction } \\
\text { increases as qc does. }\end{array}$ \\
\hline 10 & $22 / 01 / 10$ & $\begin{array}{l}\text { Test Site } \\
\text { (Virgin } \\
\text { snow) }\end{array}$ & 25 & Flat plate & $\begin{array}{l}\text { For location see photo. } \\
\text { Initial observation - less friction with } \\
\text { the flat plate. }\end{array}$ \\
\hline 11 & $22 / 01 / 10$ & $\begin{array}{l}\text { Test Site } \\
\text { (Virgin } \\
\text { snow) }\end{array}$ & 34 & standard & For location see photo. \\
\hline 12 & $22 / 01 / 10$ & $\begin{array}{l}\text { Test Site } \\
\text { (Virgin } \\
\text { snow) }\end{array}$ & $24-25$ & standard & For location see photo. \\
\hline 13 & $22 / 01 / 10$ & $\begin{array}{l}\text { 2-4 day old } \\
\text { snow in } \\
\text { snow pit }\end{array}$ & $24-25$ & standard & $\begin{array}{l}\text { Pit is } 2-3 \text { day snow }(\sim 2 \mathrm{~m}) \text { overlying } \\
\sim 1.5 \mathrm{~m} \text { of week old snow then the } \\
\text { original pit bottom. }\end{array}$ \\
\hline 14 & $22 / 01 / 10$ & $\begin{array}{l}\text { 2-4 day old } \\
\text { snow in } \\
\text { snow pit }\end{array}$ & $24-25$ & standard & $\begin{array}{l}\text { Test \#13 (probably) hit hard wall } \\
\text { hence repeated here. }\end{array}$ \\
\hline 15 & $24 / 01 / 10$ & $\begin{array}{l}\text { Vehicle } \\
\text { Park }\end{array}$ & 38 & standard & Rate testing. \\
\hline 16 & $24 / 01 / 10$ & $\begin{array}{l}\text { Vehicle } \\
\text { Park }\end{array}$ & 20 & standard & \\
\hline 17 & $24 / 01 / 10$ & $\begin{array}{l}\text { Vehicle } \\
\text { Park }\end{array}$ & 7 & standard & + dissipation test for $\sim 50$ minutes. \\
\hline
\end{tabular}




\begin{tabular}{|c|c|c|c|c|c|}
\hline 18 & $24 / 01 / 10$ & $\begin{array}{l}\text { Vehicle } \\
\text { Park }\end{array}$ & $\begin{array}{l}4 \mathrm{~mm} / \mathrm{sec}- \\
5 \mathrm{~mm} / \mathrm{sec}\end{array}$ & standard & $\begin{array}{l}\text { Switch was off for } 1^{\text {st }} \text { few seconds of } \\
2^{\text {nd }} 50 \mathrm{~cm} \text { push }\end{array}$ \\
\hline 19 & $24 / 01 / 10$ & $\begin{array}{l}\text { Vehicle } \\
\text { Park }\end{array}$ & 2 & standard & \\
\hline 20 & $24 / 01 / 10$ & $\begin{array}{l}\text { Vehicle } \\
\text { Park }\end{array}$ & 55 & standard & $\begin{array}{l}\text { Faster appears to result in less } \\
\text { resistance. }\end{array}$ \\
\hline 21 & $24 / 01 / 10$ & $\begin{array}{l}\text { 4-6 day old } \\
\text { snow in } \\
\text { snow pit }\end{array}$ & $24-25$ & standard & $\begin{array}{l}\text { Compare with Tests } 13 \text { and } 14 \text {. These } \\
\text { tests are further into the pit. } \\
\text { Remember should be two layers here } \\
\text { due two snowfall events. This test } \\
\text { should be harder due age hardening. }\end{array}$ \\
\hline 22 & 24/01/10 & $\begin{array}{l}\text { 4-6 day old } \\
\text { snow in } \\
\text { snow pit }\end{array}$ & $24-25$ & Large flat & $\begin{array}{l}\text { This one should be harder still! Does } \\
\text { this plate sense the layers earlier? } \\
\text { Should be a less 'peaky' trace. }\end{array}$ \\
\hline 23 & $24 / 01 / 10$ & $\begin{array}{l}\text { 4-6 day old } \\
\text { snow in } \\
\text { snow pit }\end{array}$ & $24-25$ & Small flat & This test should be harder than 21 . \\
\hline 24 & $25 / 01 / 10$ & $\begin{array}{l}\text { 4-6 day old } \\
\text { snow in } \\
\text { snow pit }\end{array}$ & $24-25$ & Large flat & $\begin{array}{l}\text { 2m North along pit from test 22. Is it } \\
\text { consistent with } 22 \text { ? Initial peak is but } \\
\text { not remainder. This is where I } \\
\text { climbed in and disturbed the } \\
\text { initial snow so of course it is } \\
\text { less!! }\end{array}$ \\
\hline 25 & $25 / 01 / 10$ & $\begin{array}{l}\text { Virgin } \\
\text { Snow }\end{array}$ & $24-25$ & standard & Initial o,o point. \\
\hline 26 & $25 / 01 / 10$ & $\begin{array}{l}\text { Virgin } \\
\text { Snow }\end{array}$ & $24-25$ & standard & $0.1 \mathrm{~m}$ away \\
\hline 27 & $25 / 01 / 10$ & $\begin{array}{l}\text { Virgin } \\
\text { Snow }\end{array}$ & $24-25$ & standard & 1m away \\
\hline 28 & $25 / 01 / 10$ & $\begin{array}{l}\text { Virgin } \\
\text { Snow }\end{array}$ & $24-25$ & standard & $\begin{array}{l}\text { 10m away Less disturbed sfc wind } \\
\text { crust away from the pit? Aborted at } \\
2.765 \text { due } 12 \mathrm{MPa} \text { and inability to put } \\
\text { rigid link in. Repeated below. }\end{array}$ \\
\hline 29 & $25 / 01 / 10$ & $\begin{array}{l}\text { Virgin } \\
\text { Snow }\end{array}$ & $24-25$ & standard & Repeat of above \\
\hline 30 & $25 / 01 / 10$ & $\begin{array}{l}\text { Virgin } \\
\text { Snow }\end{array}$ & $24-25$ & standard & 100m away \\
\hline 31 & $25 / 01 / 10$ & $\begin{array}{l}\text { Virgin } \\
\text { Snow }\end{array}$ & $24-25$ & standard & $1 \mathrm{~km}$ away - due East of the pit. \\
\hline 32 & $26 / 01 / 10$ & $\begin{array}{l}\text { Virgin } \\
\text { Snow @ } \\
\text { side of } \\
\text { road. }\end{array}$ & $24-25$ & standard & $\begin{array}{l}\text { These areas may have been traversed } \\
\text { in previous years? Lat/Long. }\end{array}$ \\
\hline 33 & $26 / 01 / 10$ & $\begin{array}{l}\text { Halley VI } \\
\text { Road }\end{array}$ & $24-25$ & standard & $\begin{array}{l}\text { Depth encoder stuck in } 2^{\text {nd }} 50 \mathrm{~cm} \\
\text { therefore depth is under-reading by } \\
\sim 1 \text { ocm from } \sim 70-80 \mathrm{~cm} \text { onwards. }\end{array}$ \\
\hline
\end{tabular}




\begin{tabular}{|c|c|c|c|c|c|}
\hline 34 & $26 / 01 / 10$ & $\begin{array}{l}\text { Halley VI } \\
\text { Road }\end{array}$ & $24-25$ & standard & $\begin{array}{l}\text { This is at the most traversed part of } \\
\text { the road (near barrels). Are the layers } \\
\text { due to seasonal(summer) compaction } \\
\text { - would make sense?? }\end{array}$ \\
\hline 35 & $26 / 01 / 10$ & $\begin{array}{l}\text { Virgin } \\
\text { Snow @ } \\
\text { side of } \\
\text { road. }\end{array}$ & $24-25$ & standard & $\begin{array}{l}\text { Thin (1cm) hard layer (not ice) @ 1m } \\
\text { depth identified via corer. }\end{array}$ \\
\hline 36 & $26 / 01 / 10$ & Car park & $24-25$ & standard & Dissipation test @ 1m \\
\hline 37 & $26 / 01 / 10$ & Car park & $24-25$ & standard & $\begin{array}{l}\text { To } 20 \mathrm{~m} \text {. Terminated test at } 10 \mathrm{~m} \text { due } \\
\text { excessive vibration } \mathrm{fm} \sim 5 \mathrm{~m} \text {. Friction } \\
\text { appears to dominate?? }\end{array}$ \\
\hline 38 & $27 / 01 / 10$ & Test Pit & $24-25$ & flat & $\begin{array}{l}\text { Down pre-drilled hole. Depth encoder } \\
\text { frozen - will repeat. }\end{array}$ \\
\hline 39 & $27 / 01 / 10$ & Test Pit & $24-25$ & standard & Down pre-drilled hole \\
\hline 40 & $27 / 01 / 10$ & Test Pit & $24-25$ & standard & Standard for comparison with 38,39 . \\
\hline 41 & $27 / 01 / 10$ & Test Pit & $24-25$ & standard & $\begin{array}{l}\text { Standard for comparison with } 38,39 . \\
\text { Depth encoder stuck at } \sim 13.5 \mathrm{~cm} \\
\text { therefore repeated in } 42 .\end{array}$ \\
\hline 42 & $27 / 01 / 10$ & Test Pit & $24-25$ & standard & As abv. \\
\hline 43 & & $\begin{array}{l}\text { Cyclic } \\
\text { Testing }\end{array}$ & & & $\begin{array}{l}\text { Didn't really work - used dissipation } \\
\text { test as going up and down. But, note } \\
\text { shallow hard layer in carpark. Is it } \\
\text { getting harder - age hardening?? }\end{array}$ \\
\hline 44 & & $\begin{array}{l}\text { Beside } \\
\text { Skiway }\end{array}$ & & standard & \\
\hline 45 & & Skiway & & standard & \\
\hline 46 & & Skiway & & standard & \\
\hline 47 & $31 / 01 / 10$ & $\begin{array}{l}\text { Martin's } \\
\text { cutting }\end{array}$ & & standard & $\begin{array}{l}\text { Side of snow cutting to act as baseline } \\
\text { for \#48 }\end{array}$ \\
\hline 48 & $31 / 01 / 10$ & $\begin{array}{l}\text { Martin's } \\
\text { cutting }\end{array}$ & & standard & $\begin{array}{l}\text { Metal plate inserted at depth of } 38 \mathrm{~cm} \\
\text { To see if cone senses?? Drove cone to } \\
37.5 \mathrm{~cm} \text {. }\end{array}$ \\
\hline 49 & $31 / 01 / 10$ & $\begin{array}{l}\text { Martin's } \\
\text { cutting }\end{array}$ & & Flat cone & $\begin{array}{l}\text { As abv but with flat to see if } \\
\text { compaction zone effects it. }\end{array}$ \\
\hline 50 & $31 / 01 / 10$ & $\begin{array}{l}\text { Martin's } \\
\text { cutting }\end{array}$ & & flat & $\begin{array}{l}\text { Higher up cutting to assess 'sensing' } \\
\text { of ice layers. Thin }(1 \mathrm{~cm}) \text { ice layer at } \\
\sim 1.9 \mathrm{~m}, \text { multiple ice from } \sim 2.5 \mathrm{~m} \text {. }\end{array}$ \\
\hline 51 & $31 / 01 / 10$ & $\begin{array}{l}\text { Martin's } \\
\text { cutting }\end{array}$ & & standard & $\begin{array}{l}\text { Higher up cutting to assess 'sensing' } \\
\text { of ice layers. Thin }(1 \mathrm{~cm}) \text { ice layer at } \\
\sim 1.9 \mathrm{~m}, \text { multiple ice from } \sim 2.5 \mathrm{~m} \text {. }\end{array}$ \\
\hline 52 & $31 / 01 / 10$ & $\begin{array}{l}\text { Martin's } \\
\text { cutting }\end{array}$ & & standard & $\begin{array}{l}\text { On top of cavern, testing response } \\
\text { upon probing into 'space' }+ \\
\text { dissipation test at } \sim 1 \mathrm{~m} \text {. }\end{array}$ \\
\hline 53 & $31 / 01 / 10$ & Martin's & & standard & This test to verify stratigraphy after \\
\hline
\end{tabular}




\begin{tabular}{|c|c|c|c|c|c|}
\hline & & cutting & & & $\begin{array}{l}\text { noting marked decrease in resistance } \\
\text { post DT in abv test \# } 52 \text {. Compare the } \\
\text { two and verify that upper } \\
\text { stratigraphy/resistance is essentially } \\
\text { the same - that there is a real drop in } \\
\text { resistance. Yes, drop occurred before } \\
\text { D. Test. }\end{array}$ \\
\hline 54 & $31 / 01 / 10$ & $\begin{array}{l}\text { Martin's } \\
\text { cutting }\end{array}$ & & standard & $\begin{array}{l}\text { Inclined test to get into pit. See } \\
\text { photos. Nope. Went to } \sim 8 \mathrm{~m} \text { and } \\
\text { didn't get there. Concerned about } \\
\text { bending rods. }\end{array}$ \\
\hline 55 & $31 / 01 / 10$ & $\begin{array}{l}\text { Adjacent to } \\
\text { skiway }\end{array}$ & 57 & standard & $\begin{array}{c}\text { Test towards plate at ski way (virgin } \\
\text { snow nearby). Cone was at } 4 \mathrm{~cm} \text { depth } \\
\text { at test start so must add } 4 \mathrm{~cm} \text { to } \\
\text { depths. }\end{array}$ \\
\hline 56 & $31 / 01 / 10$ & $\begin{array}{l}\text { Adjacent to } \\
\text { skiway }\end{array}$ & 25 & standard & $\begin{array}{c}\text { As abv - different rate. Started } \sim 2 \mathrm{~cm} \\
\text { below and chuck slipped. }\end{array}$ \\
\hline 57 & $31 / 01 / 10$ & $\begin{array}{l}\text { Adjacent to } \\
\text { skiway }\end{array}$ & 25 & standard & As abv. Cone started $\sim 2 \mathrm{~cm}$ depth. \\
\hline 58 & $01 / 02 / 10$ & Sea Ice & 25 & standard & $\begin{array}{c}\text { Reached } \sim 40 \mathrm{MPa} @ \sim 0.8 \mathrm{~m} \text { and } \\
\text { stopped! }\end{array}$ \\
\hline 59 & $01 / 02 / 10$ & $\begin{array}{l}\text { Relief } \\
\text { Route }\end{array}$ & 25 & standard & \\
\hline 60 & $04 / 02 / 10$ & $\begin{array}{l}\text { Snow Pit } \\
\quad \# 2\end{array}$ & 25 & standard & \\
\hline 61 & $04 / 02 / 10$ & $\begin{array}{l}\text { Snow Pit } \\
\quad \# 2\end{array}$ & 25 & standard & $\begin{array}{l}\text { This depth may be out by } 10 \mathrm{~cm} \text { or so } \\
\text { as DE cable was not connected at } \\
\text { start - need to adjust. }\end{array}$ \\
\hline 62 & $04 / 02 / 10$ & $\begin{array}{l}\text { Snow Pit } \\
\quad \# 2\end{array}$ & 25 & standard & \\
\hline 63 & $04 / 02 / 10$ & $\begin{array}{l}\text { Snow Pit } \\
\quad \# 2\end{array}$ & 25 & standard & \\
\hline 64 & $04 / 02 / 10$ & $\begin{array}{l}\text { Snow Pit } \\
\quad \# 2\end{array}$ & 25 & standard & \\
\hline 65 & $04 / 02 / 10$ & $\begin{array}{l}\text { Snow Pit } \\
\quad \# 2\end{array}$ & 25 & flat & \\
\hline 66 & $04 / 02 / 10$ & $\begin{array}{l}\text { Snow Pit } \\
\quad \# 2\end{array}$ & 25 & flat & \\
\hline 67 & $04 / 02 / 10$ & $\begin{array}{l}\text { Snow Pit } \\
\quad \# 2\end{array}$ & 25 & flat & \\
\hline 68 & $04 / 02 / 10$ & $\begin{array}{l}\text { Snow Pit } \\
\quad \# 2\end{array}$ & 25 & flat & \\
\hline 69 & $04 / 02 / 10$ & $\begin{array}{l}\text { Snow Pit } \\
\quad \# 2\end{array}$ & 25 & flat & \\
\hline 70 & $04 / 02 / 10$ & $\begin{array}{l}\text { Snow Pit } \\
\quad \# 2\end{array}$ & 25 & Large flat & $\begin{array}{l}\text { Went in } \sim 10 \text { mm then lifted box (no } \\
\text { rigid link). }\end{array}$ \\
\hline
\end{tabular}




\begin{tabular}{|c|c|c|c|c|c|}
\hline 71 & $07 / 02 / 10$ & $\begin{array}{l}\text { Snow Pit } \\
\quad \# 2\end{array}$ & $\begin{array}{c}0.25- \\
0.50 m m / s e \\
\text { c (need to } \\
\text { clarify) }\end{array}$ & standard & $\begin{array}{l}\text { See notes } 6 \text { Feb. } \\
\text { Very important. Some peaks } \\
\text { missed. Visually resistance dropped } \\
\text { to say o.7 but it wasn't recorded until } \\
\text { the } 5 \mathrm{~mm} \text { has passed by which time it } \\
\text { had risen back to say 1.2MPa and this } \\
\text { is the value that's recorded. This was } \\
\text { observed on a number of occasions. } \\
\text { Jolt heard in rods as weak layer } \\
\text { penetrated and resistance shows } \\
\text { weakness, but this low is not } \\
\text { necessarily recorded. @ 1.115 actual } \\
\text { max was 4.9MPa then tip decreased } \\
\text { as friction still increased. } \\
\text { + dissipation test. }\end{array}$ \\
\hline 72 & $08 / 02 / 10$ & $\begin{array}{l}\text { Snow Pit } \\
\quad \# 2\end{array}$ & 25 & Large & See text 8 Feb. \\
\hline 73 & $08 / 02 / 10$ & Car Park & 25 & Large & See text. \\
\hline 74 & $09 / 02 / 10$ & Car Park & $\begin{array}{l}\sim 0.2 \mathrm{~mm} / \mathrm{se} \\
\mathrm{c}\end{array}$ & Standard & $\begin{array}{l}\text { Why so very low resistance from } 0.2 \mathrm{~m} \\
\text { after first stop??? After each stop it } \\
\text { appears to take quite a while for the } \\
\text { resistance to build back up again. } \\
\text { Takes about } 20 \mathrm{~mm} \text { before it ramps } \\
\text { up again. What can I get from } \\
\text { friction? It's in MPa and I have } \\
\frac{\text { an area? Can I relate it to a }}{\text { shear strength etc?? }} \\
\text { Plot tip vs pore pressure - } \\
\text { correlation?? } \\
\text { + two dissipation tests. }\end{array}$ \\
\hline 75 & $10 / 02 / 10$ & Car Park & $\begin{array}{c}\sim 0.15 \mathrm{~mm} / \mathrm{s} \\
\text { ec (bit } \\
\text { slower - } \\
\text { cold } \\
\text { hydraulics? } \\
\text { ) }\end{array}$ & Standard & $\begin{array}{l}\text { Repeat of \#74 to } 1 \mathrm{~m} \text { just to test pore } \\
\text { pressure. (Different cone used.) }\end{array}$ \\
\hline $76(2 / 1)$ & $15 / 02 / 10$ & $\begin{array}{l}\text { Pressure } \\
\text { Test Site }\end{array}$ & 25 & Standard & 1m away from pressure test site. \\
\hline $77(2 / 2)$ & $15 / 02 / 10$ & $\begin{array}{l}\text { Pressure } \\
\text { Test Site }\end{array}$ & 25 & Standard & $1 \mathrm{~m}$ away from pressure test site. \\
\hline $78(2 / 3)$ & $15 / 02 / 10$ & Mound & 25 & Standard & Very hard hence terminated at $\sim 4 \mathrm{~m}$ \\
\hline $79(2 / 4)$ & $15 / 02 / 10$ & Garage & N/A & & Testing pore pressure \\
\hline $80(2 / 5)$ & $16 / 02 / 10$ & $\begin{array}{c}\text { Just } \\
\text { outside } \\
\text { perimeter }\end{array}$ & 25 & & $\begin{array}{l}\text { Looking at effect of confining } \\
\text { pressure. (Tests 8o-85) Surface test. }\end{array}$ \\
\hline $81(2 / 6)$ & $16 / 02 / 10$ & $\begin{array}{c}\text { Just } \\
\text { outside } \\
\text { perimeter }\end{array}$ & 25 & Standard & Surface test. \\
\hline
\end{tabular}




\begin{tabular}{|c|c|c|c|c|c|}
\hline $82(2 / 7)$ & $16 / 02 / 10$ & $\begin{array}{c}\text { Just } \\
\text { outside } \\
\text { perimeter }\end{array}$ & 25 & Standard & $\begin{array}{l}\text { After 0.9m surface surcharge } \\
\text { removed. }\end{array}$ \\
\hline $83(2 / 8)$ & $16 / 02 / 10$ & $\begin{array}{c}\text { Just } \\
\text { outside } \\
\text { perimeter }\end{array}$ & 25 & Standard & $\begin{array}{l}\text { After 0.9m surface surcharge } \\
\text { removed. }\end{array}$ \\
\hline $84(2 / 9)$ & $16 / 02 / 10$ & $\begin{array}{c}\text { Just } \\
\text { outside } \\
\text { perimeter }\end{array}$ & 25 & Standard & 1.3m surcharge removed. \\
\hline $85(2 / 10)$ & $16 / 02 / 10$ & $\begin{array}{c}\text { Just } \\
\text { outside } \\
\text { perimeter }\end{array}$ & 25 & Standard & 1.3m surcharge removed. \\
\hline $86(2 / 11)$ & $17 / 02 / 10$ & ZVI & 25 & Standard & $\begin{array}{c}\text { Vicinity SW Gateway (See GPR } 15 \& 16 \\
\text { too) }\end{array}$ \\
\hline $87(2 / 12)$ & $17 / 02 / 10$ & ZVI & 25 & Standard & Vicinity Garage Gateway \\
\hline $88(2 / 13)$ & $17 / 02 / 10$ & ZVI Road & 25 & Standard & $13 \mathrm{~km}$ from $\mathrm{ZV}$ \\
\hline $89(2 / 14)$ & $17 / 02 / 10$ & ZVI Road & 25 & Standard & $10 \mathrm{~km}$ from $\mathrm{ZV}$ \\
\hline $90(2 / 15)$ & $17 / 02 / 10$ & ZVI Road & 25 & Standard & $7 \mathrm{~km}$ from $\mathrm{ZV}$ \\
\hline $91(2 / 16)$ & $17 / 02 / 10$ & ZVI Road & 25 & Standard & $4 \mathrm{~km}$ from $\mathrm{ZV}(\sim 3 \mathrm{~km}$ from Perimeter $)$ \\
\hline $92(2 / 17)$ & $18 / 02 / 10$ & Nr CASLAB & 25 & Standard & For Rich's density pit. \\
\hline $93(3 / 17)$ & $18 / 02 / 10$ & Nr CASLAB & 5 & Standard & Good difference between rates. \\
\hline
\end{tabular}

Note: Friction is actual (but averaged) whereas tip will 'sense'. Therefore if friction and tip are positively correlated, friction may give a better indication of where actual layers start whereas tip will increase ('sense') before that layer is reached. Cx it. Friction will lag tip?

Note: Rigid link needs to be tightened after each push.

Note: Box not always level hence will marginally effect layer thickness (slant thickness).

Note: Whilst going through hard layers speed may briefly/instantaneously fluctuate from 20 $100 \mathrm{~mm} / \mathrm{sec}$.

Note: For tests 9-12 test start level will be higher than datum for density layer depths - need to amend. Note: Tip resistance at end of test decreases once penetration is stopped (observed to decrease from $1.2 \mathrm{MPa}$ to $\sim 0.6 \mathrm{MPa}$ )

Note: Golog receives data 2-3 times per second but only records it every $5 \mathrm{~mm}-$ OK for fast tests but not for the slow ones. 


\section{Appendix B}

\section{Standard Operating Procedures:}

Antarctic CPT 


\section{One-off tests}

\section{Mechanical}

1. Backup tractor to Box.

2. Attach Box to tractor.

3. Raise Box.

4. Move to test location.

5. Put Box on ground - ensure hydraulics completely down.

6. Turn-down tractor hydraulics to setting 3 (or less).

7. Insert rigid link and tighten.

8. Connect hydraulics to Box.

9. Connect power to Box.

10. Ensure rams go up and down.

11. Insert rod in chuck - ensure chuck is set for pushing.

12. Attach data cable to cone (through rod, and ensure threaded through other rods).

Note: Always clean the cone and cable connectors thoroughly with contact cleaner (non water based cleaner) and put a little bit of o-ring grease on the o rings to make them fully waterproof).

13. Attach cone to rod.

Note: Make sure that the cable is not twisted. To avoid this turn the cone seven times anticlockwise before screwing it to the rod, this will twist the cable in opposite direction so when the cone is fully screwed to the rod the cable will get fully untwisted.

\section{Electrical}

1. Attach data cable to Golog Box.

2. Mount and connect depth encoder (DE).

3. Turn on Golog Box and laptop.

4. Open Gonsite.

5. Enter cone calibration data (via disk/USB) if not already done.

6. Choose 'Testing'.

7. Select Cone, 'tick' cone, OK. 
8. Select layout (\#4).

9. Select F2 - choose file location.

\section{Conduct Test}

1. Ensure cone has equilibrated for temperature for $1 \mathrm{hr}$ (in air or snow).

\section{Select 'Start'}

3. Note down offsets and OAT.

Note: Observe the fluctuations for a couple of minutes; this will give you important information about the stability of the measuring system and the cone integrity. If the values oscillate too much it will likely be caused by a bad connection ( $90 \%$ of the cases). So troubleshooting:

1. Clean the connectors again and plug them in properly, if still nogo then

2. Use another cable, if still nogo then

3. Try a new cone, then

4. Problem may be inside Golog box - contact Roi.

Note: You should observe the readings for every channel when the rig is on deck prior to deployment. The Oscillations in every channel should be lower than:

1. TIP: $40 \mathrm{OPa}$

2. SLEEVE: $8 \mathrm{kPa}$

3. PORE PRESSURE: $8 \mathrm{kPa}$

4. Ensure

a. DE connected.

b. DE switched 'ON'

5. Select 'OK'

6. Push hydraulic lever down and start test.

7. After $0.7 \mathrm{~m}$ :
a. $\mathrm{DE}$ 'OFF'
b. Connect new rod
c. Raise rams
d. DE 'ON'
e. Continue test.

8. Repeat above till required depth.

9. Once at required depth:
a. $\mathrm{DE}$ 'OFF'
b. Raise and remove rods until cone at surface
c. Select 'STOP'

10. Note down offsets. 
11. Power-off computer and Golog box.

12. Disconnect power and hydraulics.

13. Coil cable.

\section{Remove rigid link.}

15. Raise box and move away.

\section{$\underline{\text { Note }}$}

1. No data is recorded unless the DE senses movement.

2. Don't push a cone unless $\mathrm{DE}$ is ' $\mathrm{ON}$ '

\section{Turn 'OFF' DE before raising rams.}

\section{Consecutive tests}

\section{Mechanical}

16. Backup tractor to Box.

17. Attach Box to tractor.

18. Raise Box.

19. Move to test location.

20. Put Box on ground - ensure hydraulics completely down.

\section{Turn-down tractor hydraulics to setting 3 (or less).}

22. Insert rigid link and tighten.

23. Connect hydraulics to Box.

24. Connect power to Box.

25. Ensure rams go up and down.

26. Insert rod in chuck - ensure chuck is set for pushing.

27. Attach data cable to cone (through rod, and ensure threaded through other rods). 28. Attach cone to rod.

\section{$\underline{\text { Electrical }}$}

1. Attach data cable to Golog Box.

2. Mount and connect depth encoder (DE). 
3. Turn on Golog Box and laptop.

4. Open Gonsite.

5. Enter cone calibration data (via disk/USB) if not already done.

6. Choose 'Testing'.

7. Select Cone, 'tick' cone, OK.

8. Select layout (\#4).

9. Select F2 - choose file location.

\section{Conduct Test}

1. Ensure cone has equilibrated for temperature for $1 \mathrm{hr}$ (in air or snow).

2. Select 'Start'

3. Note down offsets and OAT.

Note: Observe the fluctuations for a couple of minutes; this will give you important information about the stability of the measuring system and the cone integrity. If the values oscillate too much it will likely be caused by a bad connection ( $90 \%$ of the cases). So troubleshooting:

1. Clean the connectors again and plug them in properly, if still nogo then

2. Use another cable, if still nogo then

3. Try a new cone, then

4. Problem may be inside Golog box - contact Roi.

Note: You should observe the readings for every channel when the rig is on deck prior to deployment. The Oscillations in every channel should be lower than:

1. TIP: $40 \mathrm{kPa}$

2. SLEEVE: $8 \mathrm{kPa}$

3. PORE PRESSURE: $8 \mathrm{kPa}$

4. Ensure

a. DE connected.

b. DE switched 'ON'

5. Select 'OK'

6. Push hydraulic lever down and start test.

7. After $0.7 \mathrm{~m}$ :
a. $\mathrm{DE}$ 'OFF'
b. Connect new rod
c. Raise rams
d. DE 'ON'
e. Continue test. 
8. Repeat above till required depth.

9. Once at required depth:
a. $\mathrm{DE}$ 'OFF'
b. Raise and remove rods until cone at surface
c. Select 'STOP'

10. Note down offsets.

\section{Remove rigid-link.}

12. Raise Box.

13. Move to next site.

14. Lower box.

\section{Ensure tractor hydraulics at setting 3 (or less).}

16. Insert rigid-link and tighten.

17. Return to Electrical \#9 and repeat, else if finished

18. Power-off computer and Golog box.

19. Disconnect power and hydraulics.

20. Coil cable.

\section{Remove rigid link.}

22. Raise box and move away.

\section{Data Management}

1. Files accessed via shortcut on desktop.

2. Input into Open Office and then manipulate.

3. Columns are:
I. Depth (m)
II. $\mathrm{q}_{\mathrm{c}}(\mathrm{MPa})$
III. Friction ratio (MPa)
IV. Pore Pressure $(\mathrm{kPa})$
V. Rate $(\mathrm{cm} / \mathrm{sec})$
VI. Time (sec) 


\section{Appendix C}

Calculation of maximum

penetrative force via Johnson's

(2003) statistical micromechanical method 


\title{
NOMENCLATURE
}

\author{
$A_{\mathrm{b}} \quad$ The base area of a cone penetrometer, $A_{\mathrm{b}}=\pi r_{\mathrm{bmax}}^{2}$. \\ $A_{\text {s }} \quad$ The penetrometer effective surface area. \\ c The cohesion strength of microstructural elements for a Mohr- \\ Coulomb failure criteria. \\ $f_{\perp \mathrm{r}} \quad$ The microstructural element failure force normal to the \\ penetrometer surface. \\ $f_{\mu \mathrm{r}} \quad$ The force of friction between a penetrometer surface and micro- \\ structural elements or the compacted granular material (soil/ \\ penetrometer friction).
}

$\bar{f}_{\perp}$ The average microstructural element reaction force normal to the penetrometer surface.

$f_{\mathrm{pr}} \quad$ The component of the microstructural element failure force directed along the axis of cone penetration.

$\bar{f}_{\mathrm{p}} \quad$ The average microstructural element reaction force directed along the axis of cone penetration.

$F_{\mathrm{pm}} \quad$ The maximum penetration force for a cone penetrometer.

$F_{\beta} \quad$ The influence path function that defines the influence of $P_{\mathrm{cm} 2}$ on the total probability of contact, $P_{\mathrm{c}}$.

$k_{\perp} \quad$ The coefficient of elasticity normal to the penetrometer surface for a microstructural element (related to the microstructural elastic modulus by $E_{\perp}=k_{\perp} L_{\perp} / L_{\|}^{2}$ ).

$L_{1} \quad$ The microstructural element average dimension along the axis of penetration.

$L_{2} \quad$ The microstructural element average dimension normal to the axis of penetration.

$L_{\|} \quad$ The microstructural element average dimension parallel to the penetrometer surface.

$L_{\perp} \quad$ The microstructural element average dimension perpendicular to the penetrometer surface.

$n \quad$ The number of failed microstructural element layers that have been traversed by the penetrometer surface (Fig. 2). 
$\bar{N}_{\mathrm{c}} \quad$ The average number of microstructural elements in contact with the penetrometer effective surface.

$N_{\mathrm{cm}} \quad$ The maximum number of microstructural elements in contact with the penetrometer effective surface.

$N_{\mathrm{s}} \quad$ The number of microstructural elements adjacent to the penetrometer effective surface.

$P \quad$ The effective normal stress acting on the failure surface of a microstructural element for the Mohr-Coulomb failure criteria.

$P_{\mathrm{c}} \quad$ The probability of contact for a microstructural element that is adjacent to the penetrometer effective surface.

$P_{\mathrm{cm} 1} \quad$ The maximum probability of contact between microstructural elements and the penetrometer effective surface.

$P_{\mathrm{cm} 2} \quad$ The added probability of contact between microstructural elements and the penetrometer effective surface due to compaction that fills the pore space of intact particles and the surface roughness of microstructural elements, $\Delta r$.

PES Penetrometer effective surface: The cone surface or, when material compaction occurs, the interface between the compaction zone and surrounding microstructural elements (Fig. 1a and b).

$R_{\mathrm{pm}} \quad$ The maximum penetration resistance for a cone penetrometer, $R_{\mathrm{pm}}=F_{\mathrm{pm}} / A_{\mathrm{b}}$.

$r_{\mathrm{b}} \quad$ The base radius of that portion of the cone penetrometer in contact with the granular material, $r_{\mathrm{b}}=Z \sin \theta \cos \theta$ (Fig. 1).

$r_{\mathrm{bmax}} \quad$ The base radius for a cone penetrometer.

$r_{\mathrm{i}}, r_{\mathrm{f}} \quad$ The radii defining the back (inner) edge, $r_{\mathrm{i}}$, and front (outer) edge, $r_{\mathrm{f}}$, of the compaction zone around a penetrometer (Fig. 1).

$r_{\text {imax }}, r_{\text {fmax }}$ The radii defining maximum values of $r_{\mathrm{i}}, r_{\mathrm{f}}$ when the penetrometer is fully engaged with the granular material (Fig. 1).

$r_{\beta} \quad$ The radius of the penetrometer plus the compaction zone about the penetrometer at $r_{\mathrm{b}}$ (Fig. 1).

$r_{\beta \max } \quad$ The total radius of the penetrometer plus the compaction zone around the penetrometer when it is fully engaged with the granular material (Fig. 1). 
$\Delta r \quad$ The maximum dimension of the geometric surface roughness of microstructural elements (Fig. 1b).

Z The cone penetration depth (Fig. 1).

$Z_{\max } \quad$ The penetration depth at which a cone penetrometer is fully engaged with a granular material (Fig. 1a).

$T_{\beta} \quad$ The thickness of the layer of compacted broken fragments or particles created by microstructural element failure $\left(r_{\mathrm{f}}-r_{\mathrm{i}}\right)$.

$\beta \quad$ The compaction volume strain of broken fragments defined as $(1-\rho 0 / \rho)$.

$\beta_{\mathrm{cr}} \quad$ The critical compaction coefficient defined as the compaction at which broken fragments lock up and particle rearrangement ceases.

$\delta_{\perp \mathrm{r}} \quad$ Microstructural element deflection at failure.

$\phi \quad$ The angle of internal friction for microstructural elements used to define the Mohr-Coulomb failure criteria.

$\gamma, \gamma_{\mathrm{cr}} \quad$ The compaction angle and critical compaction angle that defines the zone of compacted material around a cone penetrometer (Fig. 1).

$\mu \quad$ The coefficient of friction between the penetrometer and the compacted granular material (soil/penetrometer friction coefficient).

$\theta \quad$ The cone penetrometer half-angle.

$\rho_{0} \quad$ The initial density of the granular material.

$\rho \quad$ The material density in the compaction zone during compaction.

$\rho_{\text {cr }} \quad$ The critical material density when a granular material locks up and further compaction through particle rearrangement no longer occurs.

$\tau \quad$ The shear stress on the Mohr-Coulomb failure plane between a microstructural element and its neighbors.

$\sigma_{N_{c}}^{2} \quad$ The variance about the average number of microstructural elements in contact with the penetrometer effective surface, $\bar{N}_{\mathrm{c}}$. 
Johnson (2003) derived the following expression for the maximum cone penetration force:

$$
F_{\mathrm{pm}}=\frac{f_{\perp \mathrm{r}}(\sin \theta+\mu \cos \theta) A_{\mathrm{b}}}{2 \beta_{\mathrm{cr}} \sin \left(\gamma_{\mathrm{cr}}+\theta\right) L_{\|}^{2}} P_{\mathrm{c}}\left(1+3 \sqrt{\frac{\beta_{\mathrm{cr}} \sin \left(\gamma_{\mathrm{cr}}+\theta\right) L_{\|}^{2}\left(1-P_{\mathrm{c}}\right)}{A_{\mathrm{b}} P_{\mathrm{c}}}}\right)
$$

Figure C.1: Expression for maximum cone penetration force via statistical micromechanical reasoning.

This expression was used with the values tabled below to generate values discussed in Chapter 7.

Table C.1: Values used in Johnson's physical expression for the maximum cone penetration force.

\begin{tabular}{|c|c|}
\hline Parameter & Value \\
\hline$F_{p m}$ & To be determined. \\
\hline$f_{\perp r}$ & $48 \mathrm{~N}$ \\
\hline$\theta$ & $30^{\circ}$ \\
\hline$\mu$ & 0.1 \\
\hline$\beta_{c r}$ & 0.2727 \\
\hline$\gamma_{c r}$ & $28^{\circ}$ \\
\hline$L_{\|}$ & $4 \mathrm{~mm}$ \\
\hline$A_{b}$ & $10 \mathrm{~cm}^{2}$ \\
\hline$P_{c}$ & 0.001 \\
\hline
\end{tabular}

